KEK-TH-375

KEK preprint 93-159

KANAZAWA-94-19

DESY 94-002

YUMS 94-22

SNUTP 94-82

September 1994

\title{
A Novel Approach to Confront Electroweak Data and Theory
}

\author{
K. Hagiwara, S. Matsumotof, \\ Theory Group, KEK, Tsukuba, Ibaraki 305, Japan \\ D. Haidt \\ DESY, Notkestraße 85, D-22603 Hamburg, Germany \\ and \\ C.S. Kim \\ Department of Physics, Yonsei University, Seoul 120-749, Korea
}

\begin{abstract}
A novel approach to study electroweak physics at one-loop level in generic $\mathrm{SU}(2)_{\mathrm{L}} \times \mathrm{U}(1)_{\mathrm{Y}}$ theories is introduced. It separates the 1-loop corrections into two pieces: process specific ones from vertex and box contributions, and universal ones from contributions to the gauge boson propagators. The latter are parametrized in terms of four effective form factors $\bar{e}^{2}\left(q^{2}\right), \bar{s}^{2}\left(q^{2}\right), \bar{g}_{Z}^{2}\left(q^{2}\right)$ and $\bar{g}_{W}^{2}\left(q^{2}\right)$ corresponding to the $\gamma \gamma, \gamma Z, Z Z$ and $W W$ propagators. Under the assumption that only the Standard Model contributes to the process specific corrections, the magnitudes of the four form factors are determined at $q^{2}=0$ and at $q^{2}=m_{Z}^{2}$ by fitting to all available precision experiments. These values are then compared systematically with predictions of $\mathrm{SU}(2)_{\mathrm{L}} \times \mathrm{U}(1)_{\mathrm{Y}}$ theories. In all fits $\alpha_{s}\left(m_{Z}\right)$ and $\bar{\alpha}\left(m_{Z}^{2}\right)$ are treated as external parameters in order to keep the interpretation as flexible as possible. The treatment of the electroweak data is presented in detail together with the relevant theoretical formulae used to interpret the data. No deviation from the Standard Model has been identified. Ranges of the top quark and Higgs boson masses are derived as functions of $\alpha_{s}\left(m_{Z}\right)$ and $\bar{\alpha}\left(m_{Z}^{2}\right)$. Also discussed are consequences of the recent precision measurement of the left-right asymmetry at SLC as well as the impact of a top quark mass and an improved $W$ mass measurement.
\end{abstract}

To be published in Zeitschrift für Physik C

${ }^{*}$ On leave from Department of Physics, Kanazawa University, Kanazawa, 920-11, Japan 


\section{Introduction}

The Standard Model (SM) of the electroweak interactions has been with us for nearly two decades. Despite the general belief that it should be an effective theory valid at energies below the Fermi scale, so far no unambiguous sign of physics beyond the SM has been found nor any clue to the origin of the underlying gauge symmetry breaking mechanism. On the other hand, the accuracy of the experiments testing the electroweak theory has improved significantly in the past decade both in low energy neutral current experiments and in high energy collider experiments on the $W$ and $Z$ boson properties. The precision of these experiments has reached the level, where meaningful searches for new physics through the investigation of quantum effects can be carried out.

The effects may be significant, if there are new particles with masses as light as weak bosons, or if many new particles contribute constructively, or if there exist new strong interactions among them. Even in the absence of such a signal, constraints on certain new physics possibilities can be derived and tightened in future precision experiments. With this motivation to study electroweak radiative corrections several groups have made efforts towards comprehensive and systematic analyses [1] [18].

In this report a novel approach to confront electroweak data and theory is presented with the aim of a systematic look for new physics effects. In the following, the conditions imposed on the electroweak analysis scheme are outlined.

Since it is the aim to search for new physics effects in the electroweak precision data, a model-independent framework to analyse the data is required. As both the experimental accuracy and the new physics effects looked for are of similar size as the SM radiative effects, it is essential to take account of the SM radiative effects as accurately as possible. For testing grand unification of the three gauge couplings [19 23] the fits should be studied quantitatively as a function of $\alpha_{s}$. Furthermore, the level of precision accessible in the near future is such that the present uncertainty in the hadronic vacuum polarization contribution to the running of the effective QED coupling constant $\alpha\left(q^{2}\right)$ severely limits the ability to study new physics through quantum effects. In order to assess the effects of possible future improvements in the $e^{+} e^{-}$hadroproduction experiments at low and intermediate energies, the consequences of varying $\alpha\left(m_{Z}^{2}\right)$ should be examined quantitatively. During the course of this study, sometimes the published results of earlier theoretical analysis could not be reproduced easily. This happened in most cases because not all the details of the assumptions and approximations underlying the analysis have been clearly stated in the literature. The quantum effects studied are so sensitive to details of the exact treatment of higher order effects and to uncertainties in the analysis that equally sensible looking assumptions often lead to a significant numerical difference. We therefore make 
every effort to render the report self-contained so that all our results can be reproduced unambiguously.

In order to comply with all the above requirements, our comprehensive analysis of electroweak precision experiments is performed according to the following steps, by systematically strengthening the underlying theoretical assumptions.

1: All electroweak data are expressed in terms of model-independent parameters.

For the choice of model-independent parameters, we basically follow the strategy of ref. [24] for low energy neutral current experiments, and that of the LEP electroweak working group [3] for the $Z$ parameters. In addition, the $W$ boson mass, the fine structure constant $\alpha$ and the Fermi coupling constant $G_{F}$ are used as inputs of the analysis. Some of these parameters are directly related to experimental observables up to corrections due to known physics, such as the external QED bremsstrahlung effects and the quark-parton model, and uncertainties in these correction factors are included as part of the errors of the experimentally measured parameters.

2: The model-independent parameters are then expressed in terms of the pole positions of the $W$ and $Z$ propagators, and the $S$-matrix elements of four external fermions, quarks or leptons, which are approximated as products of two external standard $V \pm A$ currents and the scalar transition form factors.

All electroweak precision measurements that have been performed so far can be expressed in terms of the $S$-matrix elements of quark and lepton processes whose masses are negligible compared to the weak boson masses. To an excellent approximation, chirality-flip terms in the loop amplitudes can be neglected and the relevant $S$-matrix elements can be expressed in terms of the scalar product of the standard $V \pm A$ currents multiplied by transition form factors depending on the flavors and chiralities of the currents as well as the momentum transfer of the process under consideration. External QCD and QED corrections can hence be applied exactly as in the SM, and electroweak models can be confronted with experiment, once the transition form factors are determined in a particular model. The dependence of the fit on the QCD parameter $\alpha_{s}$ and quark masses is taken into account by introducing appropriate external parameters. Up to this stage, our analysis is quite general, as the formulae are valid for any electroweak model respecting the flavor and chirality conservation laws of the SM, that is, for all new physics contributions which can interfere with the leading SM amplitudes.

Although one may attempt to constrain these model-independent transition form factors directly by experiment, we find it impractical, since the number of independent 
transition form factors exceeds by far the effective number of degrees of freedom provided by precision measurements. Hence, we perform the quantitative comparison of data with theory in a more restricted class of models which are minimal extensions from the SM, i.e. those models which respect the $\mathrm{SU}(2)_{\mathrm{L}} \times \mathrm{U}(1)_{\mathrm{Y}}$ gauge symmetry broken spontaneously down to $\mathrm{U}(1)_{\mathrm{EM}}$.

3: The transition form factors are expanded perturbatively in $\mathrm{SU}(2)_{\mathrm{L}} \times \mathrm{U}(1)_{\mathrm{Y}}$ gauge couplings, and radiative effects are classified either as the universal gauge boson propagator corrections or as the process specific vertex and box corrections. The universal propagator correction factors are then parameterized by four charge form factors, $\bar{e}^{2}\left(q^{2}\right), \bar{s}^{2}\left(q^{2}\right), \bar{g}_{Z}^{2}\left(q^{2}\right)$ and $\bar{g}_{W}^{2}\left(q^{2}\right)$, corresponding to the $\gamma \gamma, \gamma Z, Z Z$ and $W W$ propagator degrees of freedom.

The restriction to the electroweak gauge group $\mathrm{SU}(2)_{\mathrm{L}} \times \mathrm{U}(1)_{\mathrm{Y}}$ implies at the tree level that all fermions, quarks and leptons, couple to the electroweak gauge bosons universally with the same coupling constant as long as they have common $\mathrm{SU}(2)_{\mathrm{L}} \times \mathrm{U}(1)_{\mathrm{Y}}$ quantum numbers. This universality of the gauge boson coupling to quarks and leptons can in general be violated at the quantum level, because the gauge symmetry breaks spontaneously down to $\mathrm{U}(1)_{\mathrm{EM}}$. It has been widely recognized, however, that this universality of the couplings holds true even at one-loop level in a wider class of models where new particles affect the precision experiments only via their effects on the electroweak gauge boson propagators [1 [10]. This class of new physics effects is often called oblique [1, [1] or propagator [7] corrections, or those satisfying generalized universality [10]. This concept of universality can be generalized to certain vertex corrections with non-standard weak boson interactions [11]. It is also often useful in theories with non-standard vertex and box corrections, such as the supersymmetric SM (SUSY-SM), since the propagator corrections can be larger than the vertex/box ones: propagator corrections can be significant either because of a large multiplicity of contributing particles or by the presence of a relatively light new particle.

When confronting the electroweak theory with experiment, we adopt this distinction between new physics contributions to the gauge boson propagators and those to the rest, where we allow the most general contributions in the former, whereas we consider only the SM contributions to the latter (vertex and box corrections).

4: By assuming that the well-known SM contributions dominate the process specific vertex and box corrections, apart from the $Z b_{L} b_{L}$ vertex for which new physics contributions are allowed, we determine from precision experiments the four universal charge form factors at the typical momentum transfer scales, $q^{2}=0$ and $m_{Z}^{2}$. 
The new physics contributions may either prevent our ability to fit the experimental data within our approach, or lead to non-standard values of the fitted four charge form factors and the $Z b_{L} b_{L}$ vertex form factor, $\bar{\delta}_{b}\left(q^{2}\right)$. At this stage, the whole body of electroweak precision data can be expressed in terms of the two weak boson masses $m_{W}$ and $m_{Z}$, and these five form factors, that is, the four universal charge form factors and $\bar{\delta}_{b}\left(q^{2}\right)$. Although the form factors could be determined at any point on the momentum scale $q^{2}$, they are actually measured with adequate precision only at two specific $q^{2}$ ranges, namely all four charge form factors at $q^{2}=0$ or $q^{2} \ll m_{Z}^{2}$, while $\bar{s}^{2}\left(q^{2}\right), \bar{g}_{Z}^{2}\left(q^{2}\right)$ and $\bar{\delta}_{b}\left(q^{2}\right)$ at $q^{2}=m_{Z}^{2}$. Hence, there are just 9 electroweak parameters measured accurately enough to be used for testing theories: $m_{W}$ and $m_{Z}, \bar{e}^{2}(0), \bar{s}^{2}(0), \bar{g}_{Z}^{2}(0)$ and $\bar{g}_{W}^{2}(0), \bar{s}^{2}\left(m_{Z}^{2}\right), \bar{g}_{Z}^{2}\left(m_{Z}^{2}\right)$ and $\bar{\delta}_{b}\left(m_{Z}^{2}\right)$.

Apart from the vertex form factor $\bar{\delta}_{b}\left(m_{Z}^{2}\right)$ the remaining 8 parameters characterise the universal propagator corrections. On the experimental side, the three quantities $\alpha$, $G_{F}$ and $m_{Z}$ are measured so accurately that it is justifiable to treat them as constants: $\alpha=1 / 137.0359895$ and $G_{F}=1.16639 \times 10^{-5} \mathrm{GeV}^{-2}$ from the PDG listing [25], and $m_{Z}=91.187 \mathrm{GeV}$ from the LEP results [26]. Among the 8 universal parameters above, $\bar{e}^{2}(0)=4 \pi \alpha$ and $m_{Z}$ are fixed immediately, while $G_{F}$ fixes the ratio $\bar{g}_{W}^{2}(0) / m_{W}^{2}$, once we assume the SM dominance of the vertex and box corrections $\left(\bar{\delta}_{G}\right)$ to the muon decay lifetime. Since the gauge boson properties are fixed at tree level by only three parameters in general models with the $\mathrm{SU}(2) \times \mathrm{U}(1)$ symmetry broken by a vacuum expectation value, the remaining 5 universal parameters serve to test the theory at the quantum level (see Table 1 ). We therefore first determine from precision experiments the 5 parameters, $\bar{s}^{2}(0), \bar{g}_{Z}^{2}(0), \bar{g}_{W}^{2}(0), \bar{s}^{2}\left(m_{Z}^{2}\right)$ and $\bar{g}_{Z}^{2}\left(m_{Z}^{2}\right)$, together with $\bar{\delta}_{b}\left(m_{Z}^{2}\right)$, and then confront their values with various theoretical predictions.

In the fit to the $Z$ boson parameters the strong coupling constant $\alpha_{s}\left(m_{Z}\right)$ is treated as external parameter which can be varied within certain limits. In this way the analysis remains transparent and easy to update. The fitted electroweak parameters $\bar{s}^{2}\left(m_{Z}^{2}\right)$, $\bar{g}_{Z}^{2}\left(m_{Z}^{2}\right)$ and $\bar{\delta}_{b}^{2}\left(m_{Z}^{2}\right)$ are thus presented as parametrizations in $\alpha_{s}$ (see Table 2).

When the new physics scale is significantly higher than the scale $\left(\lesssim m_{Z}^{2}\right)$ of precision measurements, new physics contributions to the running of the charge form factors can be neglected.

5: By assuming further that the running of the charge form factors between $q^{2}=0$ and $q^{2}=m_{Z}^{2}$ are governed only by SM physics, three universal parameters sensitive to radiative effects can be determined. We adopt a modified version of the $S, T, U$ parameters of ref. 四 by including the SM radiative effects as well as new physics contributions. 
Among the 5 universal parameters, the values of $\bar{s}^{2}(0) / \alpha$ and $\bar{g}_{Z}^{2}(0)$ can then be calculated from $\bar{s}^{2}\left(m_{Z}^{2}\right) / \bar{\alpha}\left(m_{Z}^{2}\right)$ and $\bar{g}_{Z}^{2}\left(m_{Z}^{2}\right)$, respectively, using SM physics only. There are then 3 remaining universal free parameters which correspond to the parameters $S, T$ and $U$ of ref. [4], $\epsilon_{1}, \epsilon_{2}$ and $\epsilon_{3}$ of ref. [7], or the other related triplets of parameters in refs. [5]. When the scale of new physics that couples to gauge boson propagators is near to the weak boson masses, its signal can be identified as an anomalous running of the charge form factors. This point has been stressed in refs. [12 in connection with the possible existence of light SUSY particles. It has also been pointed out that when new physics effects to the electroweak gauge boson sector are parametrized by the four dimension-six operators of ref. [10], there occurs anomalous running of the charge form factors [11]. The triplet parametrizations are then no longer sufficient to account for new physics degrees of freedom, and all 5 parameters in Table 1 should be regarded as free. Several alternative approaches to the same problem have been proposed in refs. [12, 14, 18].

Note that in order to obtain the charge form factors from the three known parameters $\alpha, G_{F}, m_{Z}$ and the radiative parameters $S, T$ and $U$ that are calculable in a given model, the effective QED coupling at the $Z$ mass scale, $\bar{\alpha}\left(m_{Z}^{2}\right)$ is needed. Its value is calculable from $\alpha$ in the SM but suffers from uncertainty in the hadronic vacuum polarization contribution [27 29]. The effect of this uncertainty on the final results turns out to be non-negligible. In order to gauge the effects due to this uncertainty quantitatively, we introduce $\delta_{\alpha}=1 / \bar{\alpha}\left(m_{Z}^{2}\right)-128.72$ as external parameter and allow it to vary in the fit (see Table 2). It is then straightforward to examine the effects of shifts in the $\bar{\alpha}\left(m_{Z}^{2}\right)$ value and the impact of future improvements in its measurement.

In the minimal SM, the three universal parameters $S, T, U$ and the $Z b_{L} b_{L}$ vertex form factor $\bar{\delta}_{b}\left(m_{Z}^{2}\right)$ depend on just two parameters: $m_{t}$ and $m_{H}$.

6: Finally, by assuming that no new physics contributes significantly to electroweak precision experiments, we can express all the radiative effects in terms of the two parameters of the minimal SM, $m_{t}$ and $m_{H}$. The $\chi^{2}$ curves of the global fit are shown as a function of these two parameters, for several values of $\alpha_{s}\left(m_{Z}\right)$ and $\bar{\alpha}\left(m_{Z}^{2}\right)$.

The preferred range of $m_{t}$ is presented as a function of $m_{H}, \alpha_{s}, \delta_{\alpha}$, that of $m_{H}$ as a function of $m_{t}, \alpha_{s}, \delta_{\alpha}$, that of $\alpha_{s}$ as a function of $m_{t}, m_{H}, \delta_{\alpha}$, and that of $\delta_{\alpha}$ as a function of $m_{t}, m_{H}, \alpha_{s}$. The chosen value for the parameter $\delta_{\alpha}$ is essential, since it is not well constrained by the present precision measurements alone.

A clear advantage of this approach is that we can test the electroweak theory at three qualitatively distinct levels. If we cannot fit all the data at a given $q^{2}$ with common form factor values, we should either look for new physics affecting the relevant vertex/box 
corrections significantly or else we should introduce new tree level interactions such as those induced by an exchange of a new heavy boson, or from new strong interactions that bind common constituents of quarks and leptons. If the 'universality' in terms of the above four charge form factors holds, but their $q^{2}$-dependence does not agree with the expectations of the Standard Model, we may anticipate a new physics scale very near to the present experimental limit [12], or effective higher dimensional interactions among the gauge bosons [10, 11]. New physics contributions which decouple at low energies can thus be identified by their anomalous running of the charge form factors. If the running of the form factors is found to be consistent with the SM, then our approach reduces to the standard three parameter analyses [4, [5, 0], or those with three plus one parameter [12, 14] when including the $Z b_{L} b_{L}$ vertex parameter $\bar{\delta}_{b}\left(m_{Z}^{2}\right)$ as well. Deviation from the SM is still possible, since the SM has only two free parameters, $m_{t}$ and $m_{H}$. In this case sensitivity to those new physics contributions which do not decouple at low energies remains.

As emphasized at the beginning of this section, we present at all stages of our quantitative analysis the best-fit values of the model parameters, including a parametrization of the $\chi^{2}$ goodness of the fit around its minimum as functions of the external parameters $\alpha_{s}=\alpha_{s}\left(m_{Z}\right)_{\overline{\mathrm{MS}}}$ and $\delta_{\alpha} \equiv 1 / \bar{\alpha}\left(m_{Z}^{2}\right)-128.72 \simeq\left(1 / \bar{\alpha}\left(m_{Z}^{2}\right)-1 / \alpha\right)_{\text {hadrons }}+3.88$. One can examine consequences of possible future improvements in the measurement of $\alpha_{s}$ [30] and those of hadronic contribution to $\delta_{\alpha}$ by adding to the quoted $\chi^{2}$ function terms of the form $\left[\left(\alpha_{s}-\overline{\alpha_{s}}\right) /\left(\Delta \alpha_{s}\right)\right]^{2}$ and $\left[\left(\delta_{\alpha}-\overline{\delta_{\alpha}}\right) /\left(\Delta \delta_{\alpha}\right)\right]^{2}$.

The paper is organized as follows. In section 2, we present our formalism in detail. The helicity amplitudes are stated for general four-fermion processes in terms of the universal charge form factors and process-dependent vertex and box corrections. Definitions of the form factors and the $S, T, U$ parameters are given and their SM values are shown. Section 3 contains theoretical formulae for the electroweak observables expressed in terms of the helicity amplitudes of section 2, with QCD/QED corrections. Numerical predictions are also given for wide ranges of the form factor values, and also in the minimal SM. In section 4, we present our model-independent parametrizations of all experimental data and, confront them with our theoretical predictions. The universal charge form factors and $\bar{\delta}_{b}\left(m_{Z}^{2}\right)$ are determined by assuming SM dominance in the remaining vertex and box corrections. Section 5 presents a systematic analysis of the electroweak data by gradually tightening the theoretical assumptions. First the running of the charge form factors $\bar{g}_{Z}^{2}\left(q^{2}\right)$ and $\bar{s}^{2}\left(q^{2}\right)$ is tested, then the 4-parameter $\left(S, T, U\right.$ and $\left.\bar{\delta}_{b}\left(m_{Z}^{2}\right)\right)$ fit to all electroweak data is performed by assuming SM running of the charge form factors. Finally, constraints on $m_{t}$ and $m_{H}$ are discussed in the SM fit. The total $\chi^{2}$ of the SM is parametrized in terms of $m_{t}, m_{H}, \alpha_{s}$ and $\delta_{\alpha}$. In section 6 , consequences of the new precision measurement 
of the left-right asymmetry [31] and the impact of a top quark mass measurement are considered. Section 7 summarizes our observations. Details of the theoretical formulae used are collected in the appendices. In appendix A, we give all the SM radiative correction terms completely at one-loop level, and partly at two-loop level for $O\left(\alpha \alpha_{s}\right)$ terms. They are classified into three parts, the propagator corrections, the vertex corrections and the box corrections. In appendix B, we discuss the renormalization group improvement of the charge form factors and hadronic contributions to the gauge boson propagators. Appendix $\mathrm{C}$ gives the complete analytical formulae for the $S, T, U$ parameters and the $Z b_{L} b_{L}$ vertex form factor $\bar{\delta}_{b}\left(m_{Z}^{2}\right)$ in the SM. Here all the known two-loop level corrections are included. We also give convenient approximations to the exact formulae. Appendix D provides explicit expressions for the $A, B, C, D$ functions 32 that are used to express all the one-loop correction factors.

\section{Basic formalism}

\subsection{S-matrix elements, weak boson masses, and charge form factors}

All the precision experiments sensitive to electroweak physics at one-loop level so far are concerned with processes involving external fermions, that is, leptons or quarks (excluding top quarks), whose masses can safely be neglected in the correction terms as compared to the weak boson masses. There are the $Z$ boson properties as measured at LEP and SLC, the neutral current $(N C)$ processes at low energies $\left(\ll m_{Z}\right)$, the measurements of charged current $(C C)$ processes at low energies and those of the $W$ mass at $p \bar{p}$ colliders. The relevant observables in these processes are then expressed in terms of the $S$-matrix elements of four external fermions which form a scalar product of two chirality conserving currents. All the information on electroweak physics is contained in the scalar amplitudes which multiply these current-current products.

For example, consider the $S$-matrix element responsible for the generic 4-fermion $N C$ process $i j \rightarrow i j$ (or any one of its crossed channels). This includes $e^{+} e^{-} \rightarrow f \bar{f}$ as well as $\nu_{\mu} q \rightarrow \nu_{\mu} q$. The matrix element has the form

$$
T_{i j}=M_{i j} J_{i} \cdot J_{j}
$$

where $J_{i}^{\mu}$ and $J_{j}^{\mu}$ denote currents without coupling factors, that is, $J_{i}^{\mu}=\bar{\psi}_{f} \gamma^{\mu} P_{\alpha} \psi_{f}$ for $i=f_{\alpha}$, where $P_{\alpha}=\left(1+\alpha \gamma_{5}\right) / 2$ with $\alpha= \pm 1$ are the chiral projectors.1 All radiative

\footnotetext{
${ }^{1}$ We use the chirality index $\alpha=+1$ for right-handedness and $\alpha=-1$ for left-handedness
} throughout the paper; e.g., $P_{+1}=P_{+}=P_{R}$ and $P_{-1}=P_{-}=P_{L}$ for the chiral projectors, $f_{+}=f_{R}$ and $f_{-}=f_{L}$ for chiral fermions. 
effects interfering with the tree-level SM amplitudes can be cast into the above form as long as terms of order $m_{f}^{2} / m_{Z}^{2}$ in the one-loop amplitudes are neglected $\left(m_{f}\right.$ denoting the external fermion mass). The one-loop corrections then appear in the scalar amplitudes $M_{i j}$ which depend on flavor and chirality of the currents and on the invariant momentum transfers $s$ and $t$ of the process.

In neutral current amplitudes, the photonic corrections attached only to the external fermion lines are U(1) gauge invariant by themselves [3]. Therefore, finite and gauge invariant amplitudes can be obtained by excluding all the external photonic corrections. We find the following closed form for the generic neutral current amplitude $M_{i j}$ of (2.1) at one-loop order (see details in Appendix A) :

$$
\begin{aligned}
M_{i j}^{N C}= & \frac{Q_{i} Q_{j}}{s}\left[\bar{e}^{2}(s)+\hat{e}^{2}\left(\Gamma_{1}^{i}+\Gamma_{1}^{j}\right)(s)-i \hat{e}^{2} \Delta_{\gamma \gamma}(s)\right]+\hat{e}^{2}\left[\left(Q_{i} I_{3 j}\right) \frac{\bar{\Gamma}_{2}^{j}(s)}{s}+\left(I_{3 i} Q_{j}\right) \frac{\bar{\Gamma}_{2}^{i}(s)}{s}\right] \\
+ & \frac{1}{s-m_{Z}^{2}+i s \frac{\Gamma_{Z}}{m_{Z}} \theta(s)} \\
& \quad \times\left\{\left(I_{3 i}-Q_{i} \hat{s}^{2}\right)\left(I_{3 j}-Q_{j} \hat{s}^{2}\right)\left[\bar{g}_{Z}^{2}(s)+\hat{g}_{Z}^{2}\left(\Gamma_{1}^{i}+\Gamma_{1}^{j}\right)(s)-i \hat{g}_{Z}^{2} \Delta_{Z Z}(s)\right]\right. \\
& \quad+\left(I_{3 i}-Q_{i} \hat{s}^{2}\right) \hat{g}_{Z}^{2}\left[I_{3 j}\left(\hat{c}^{2} \bar{\Gamma}_{2}^{j}+\Gamma_{3}^{j}\right)(s)+\Gamma_{4}^{j}(s)-Q_{j}\left(\bar{s}^{2}(s)-\hat{s}^{2}+i \Delta_{\gamma Z}(s)\right)\right] \\
& \left.+\left(I_{3 j}-Q_{j} \hat{s}^{2}\right) \hat{g}_{Z}^{2}\left[I_{3 i}\left(\hat{c}^{2} \bar{\Gamma}_{2}^{i}+\Gamma_{3}^{i}\right)(s)+\Gamma_{4}^{i}(s)-Q_{i}\left(\bar{s}^{2}(s)-\hat{s}^{2}+i \Delta_{\gamma Z}(s)\right)\right]\right\} \\
+ & B_{i j}^{N C}(s, t) .
\end{aligned}
$$

Here $s$ is the momentum transfer of the current $J_{i}^{\mu}$, and $t$ is the momentum transfer between the fermions $i$ and $j$. The hatted couplings $\hat{e} \equiv \hat{g} \hat{s} \equiv \hat{g}_{Z} \hat{s} \hat{c}$ and all the ultraviolet singular loop functions are renormalized in the $\overline{\mathrm{MS}}$ scheme, and hence they depend either implicitly or explicitly on the unit-of-mass $\mu$. Three of the four charge form factors of Table $1, \bar{e}^{2}(s), \bar{s}^{2}(s)$ and $\bar{g}_{Z}^{2}(s)$, appear in the $N C$ amplitudes :

$$
\begin{aligned}
\bar{e}^{2}\left(q^{2}\right) & =\hat{e}^{2}\left[1-\operatorname{Re} \bar{\Pi}_{T, \gamma}^{\gamma \gamma}\left(q^{2}\right)\right], \\
\bar{s}^{2}\left(q^{2}\right) & =\hat{s}^{2}\left[1+\frac{\hat{c}}{\hat{s}} \operatorname{Re} \bar{\Pi}_{T, \gamma}^{\gamma Z}\left(q^{2}\right)\right], \\
\bar{g}_{Z}^{2}\left(q^{2}\right) & =\hat{g}_{Z}^{2}\left[1-\operatorname{Re} \bar{\Pi}_{T, Z}^{Z Z}\left(q^{2}\right)\right] .
\end{aligned}
$$

Imaginary parts of the propagator correction factors denoted by $\Delta_{\gamma \gamma}(s), \Delta_{\gamma Z}(s)$ and $\Delta_{Z Z}(s)$ are defined as follows :

$$
\begin{aligned}
& \Delta_{\gamma \gamma}\left(q^{2}\right)=\operatorname{Im} \bar{\Pi}_{T, \gamma}^{\gamma \gamma}\left(q^{2}\right) \\
& \Delta_{\gamma Z}\left(q^{2}\right)=\hat{s} \hat{c} \operatorname{Im} \bar{\Pi}_{T, \gamma}^{\gamma Z}\left(q^{2}\right), \\
& \Delta_{Z Z}\left(q^{2}\right)=\operatorname{Im} \bar{\Pi}_{T, Z}^{Z Z}\left(q^{2}\right)-\frac{\operatorname{Im} \bar{\Pi}_{T}^{Z Z}\left(m_{Z}^{2}\right)}{m_{Z}^{2}} .
\end{aligned}
$$


The vertex functions $\Gamma_{n}^{f_{\alpha}}(s)$ and the box functions $B_{f_{\alpha} f_{\beta}^{\prime}}(s, t)$ are process specific. The SM contributions to all the two-, three-, and four-point functions in eq. (2.2) are calculated in the 't Hooft-Feynman gauge. Their explicit forms are found in appendix A.

The residues of the $\gamma$ - and $Z$-poles are separately $\mu$-independent and gauge invariant, and therefore physical observables. For $q^{2}=0$, the vanishing of the vertex functions

$$
\Gamma_{1}^{f_{\alpha}}(0)=0, \quad \bar{\Gamma}_{2}^{f_{\alpha}}(0)=0
$$

is ensured for all $f_{\alpha}$ by the Abelian and non-Abelian parts of the Ward identities, respectively. The universal residue of the photon pole gives the square of the unit electric charge $\bar{e}^{2}(0)=4 \pi \alpha$.

Likewise, the charged current $(C C)$ process $i j \rightarrow i^{\prime} j^{\prime}$ can be expressed by

$$
M_{i j}^{C C}=\frac{1}{s-m_{W}^{2}+i s \frac{\Gamma_{W}}{m_{W}} \theta(s)}\left\{\bar{g}_{W}^{2}(s)+\hat{g}^{2}\left[\bar{\Gamma}^{i i^{\prime}}(s)+\bar{\Gamma}^{j j^{\prime}}(s)-i \Delta_{W W}(s)\right]\right\}+B_{i j}^{C C}(s, t),
$$

with an appropriate CKM factor $V_{i i^{\prime}} V_{j j^{\prime}}^{*}$ accounting for quark family mixing. The $W$ propagator corrections appear in the charge form factor $\bar{g}_{W}^{2}(s)$ and in the imaginary part $\Delta_{W W}(s)$ :

$$
\begin{aligned}
\bar{g}_{W}^{2}\left(q^{2}\right) & =\hat{g}^{2}\left[1-\operatorname{Re} \bar{\Pi}_{T, W}^{W W}\left(q^{2}\right)\right], \\
\Delta_{W W}\left(q^{2}\right) & =\operatorname{Im} \bar{\Pi}_{T, W}^{W W}\left(q^{2}\right)-\frac{\operatorname{Im} \bar{\Pi}_{T}^{W W}\left(m_{W}^{2}\right)}{m_{W}^{2}} .
\end{aligned}
$$

Factorization of the external photonic corrections does not hold for the charged current processes, and hence all the one-loop correction terms are included in eq. (2.6). Explicit forms of the SM contributions to the propagator function $\bar{\Pi}_{T}^{W W}\left(q^{2}\right)$ are found in Appendix A.

The gauge boson two-point functions appearing in eqs. (2.3), (2.4), (2.7) and (2.8) are defined as follows :

$$
\bar{\Pi}_{T, V}^{A B}\left(q^{2}\right)=\frac{\bar{\Pi}_{T}^{A B}\left(q^{2}\right)-\bar{\Pi}_{T}^{A B}\left(m_{V}^{2}\right)}{q^{2}-m_{V}^{2}},
$$

where $m_{V}$ is the physical mass of the gauge boson $V$ (that is, $m_{W}, m_{Z}$ and $m_{\gamma}$ with $\left.m_{\gamma}=0\right)$ and the subscript $T$ stands for the transverse part of the vacuum polarization tensor $\Pi_{\mu \nu}(q)$,

$$
\Pi_{\mu \nu}^{A B}(q)=\left(-g_{\mu \nu}+\frac{q^{\mu} q^{\nu}}{q^{2}}\right) \Pi_{T}^{A B}\left(q^{2}\right)+\frac{q^{\mu} q^{\nu}}{q^{2}} \Pi_{L}^{A B}\left(q^{2}\right) .
$$

Contributions from the longitudinal part of the gauge boson propagators are consistently neglected in the one-loop corrections, because they give terms of order $m_{f}^{2} / m_{V}^{2}(V=Z$ or $W$ ) in the weak amplitudes. 
The gauge boson propagators are calculated in the 't Hooft-Feynman gauge and the so-called pinch terms [2, 33, 34 of the vertex functions arising from diagrams with the weak boson self-couplings are included in the overlined functions $\bar{\Pi}_{T}^{A B}\left(q^{2}\right)$ :

$$
\begin{aligned}
\bar{\Pi}_{T}^{\gamma \gamma}\left(q^{2}\right) & =\Pi_{T}^{\gamma \gamma}\left(q^{2}\right)-\frac{\hat{e}^{2}}{4 \pi^{2}} q^{2} B_{0}\left(q^{2} ; m_{W}, m_{W}\right), \\
\bar{\Pi}_{T}^{\gamma Z}\left(q^{2}\right) & =\Pi_{T}^{\gamma Z}\left(q^{2}\right)-\frac{\hat{e} \hat{g}_{Z} \hat{c}^{2}}{4 \pi^{2}}\left(q^{2}-\frac{m_{Z}^{2}}{2}\right) B_{0}\left(q^{2} ; m_{W}, m_{W}\right), \\
\bar{\Pi}_{T}^{Z Z}\left(q^{2}\right) & =\Pi_{T}^{Z Z}\left(q^{2}\right)-\frac{\hat{g}_{Z}^{2} \hat{c}^{4}}{4 \pi^{2}}\left(q^{2}-m_{Z}^{2}\right) B_{0}\left(q^{2} ; m_{W}, m_{W}\right), \\
\bar{\Pi}_{T}^{W W}\left(q^{2}\right) & =\Pi_{T}^{W W}\left(q^{2}\right)-\frac{\hat{g}^{2}}{4 \pi^{2}}\left(q^{2}-m_{W}^{2}\right)\left[\hat{c}^{2} B_{0}\left(q^{2} ; m_{W}, m_{Z}\right)+\hat{s}^{2} B_{0}\left(q^{2} ; m_{W}, m_{\gamma}\right)\right] .
\end{aligned}
$$

Here $B_{0}$ is a Passarino-Veltman function [32] in the notation of appendix D. The overlines on the vertex functions $\bar{\Gamma}_{2}^{f_{L}}(s)$ in eqs. (2.2) and (2.6) and $\bar{\Gamma}^{i i^{\prime}}(s)$ in eq. (2.6) indicate the subtraction of the pinch term associated with this prescription (note, the pinch terms in eqs. (2.11) have a negative sign in our convention).

The absorption of the above $q^{2}$ dependent propagator-like parts of the vertex functions into the effective charges [2] improves over the usual method of absorbing the relevant vertex term at zero momentum transfer [3] in two ways. One is that the remaining vertex parts do no longer give rise to large logarithms of the type $\ln \left(-q^{2} / m_{W}^{2}\right)$ at $\left|q^{2}\right| \gg m_{W}^{2}$, and hence the effective charges are useful in making the improved Born approximation [2] even at very high energies. The second is that the effective charges are now gauge invariant [2, 34], and hence their properties can be discussed independently of the other process specific corrections of the same order. Most importantly, we can obtain explicitly renormalization group invariant relations between the $\overline{\mathrm{MS}}$ couplings and the effective charges

$$
\begin{aligned}
\frac{1}{\bar{e}^{2}\left(q^{2}\right)} & =\frac{1}{\hat{e}^{2}(\mu)}\left[1+\operatorname{Re} \bar{\Pi}_{T, \gamma}^{\gamma \gamma}\left(q^{2}\right)\right] \\
\bar{s}^{2}\left(q^{2}\right) & =\hat{s}^{2}(\mu)+\frac{\bar{e}^{2}\left(q^{2}\right)}{\hat{e}(\mu) \hat{g}_{Z}(\mu)} \operatorname{Re} \bar{\Pi}_{T, \gamma}^{\gamma Z}\left(q^{2}\right),
\end{aligned}
$$

within the 't Hooft-Feynman gauge of the electroweak theory. This enables us to discuss the renormalization group improvement of the above two effective charges as a whole, that is, without separating the contributions from the SM fermions and the rest. The trajectories of all the $\overline{\mathrm{MS}}$ couplings $\left(\hat{e}=\hat{g} \hat{s}=\hat{g}_{Z} \hat{s} \hat{c}\right)$ are completely fixed by the above two equations at one-loop level, which can be used to study quantitatively the heavy particle threshold corrections in GUT theories [21].

In the analysis presented here the $\overline{\mathrm{MS}}$ couplings act as the expansion parameters of the perturbation series, since we find them the most convenient when studying consequences 
of various theoretical models beyond the SM. Their usefulness in the SM analysis has been emphasized in ref. [35], and they are often used in the analysis of new physics contributions to the precision experiments [5]. However, it is not convenient to use the $\overline{\mathrm{MS}}$ couplings at a specific unit-of-mass $(\mu)$ scale, such as $\mu=m_{Z}$, when dealing with a theory with particles much heavier than the weak bosons because of the appearance of large logarithms of their masses. Hence, we adopt the following renormalization conditions

$$
\hat{e}^{2}=\bar{e}^{2}\left(m_{Z}^{2}\right), \quad \hat{s}^{2}=\bar{s}^{2}\left(m_{Z}^{2}\right),
$$

consistently for all processes studied. The above conditions renormalize all the logarithms of large masses with the help of the renormalization group identities (2.12) at $\left|q^{2}\right|<O\left(m_{Z}^{2}\right)$. Note that the running of $\bar{e}^{2}\left(q^{2}\right)$ and $\bar{s}^{2}\left(q^{2}\right)$ at low energies arises from the $\mathrm{QED} \times \mathrm{QCD}$ interactions [36], and hence the ratio $\bar{e}^{2}\left(q^{2}\right) / \bar{s}^{2}\left(q^{2}\right)$ is not an appropriate expansion parameter of the weak corrections at $\left|q^{2}\right| \ll m_{Z}^{2}$. Note further that, apart from details concerning the higher order terms, the effective charges $\bar{e}^{2}\left(q^{2}\right)$ and $\bar{s}^{2}\left(q^{2}\right)$ (2.12) are the same as the real parts of the corresponding star-scheme [2] charges, $e_{*}^{2}\left(q^{2}\right)$ and $s_{*}^{2}\left(q^{2}\right)$, respectively. More details on the treatment of the renormalization group improvement and the hadronic contributions to the charge form factors are given in appendix B.

Since we adopt the LEP convention [3] regarding mass and width $\left(m_{V}\right.$ and $\left.\Gamma_{V}\right)$ for both $Z$ and $W$, the Breit-Wigner propagator factors in eqs. (2.2) and (2.6) have the running width factor, and the imaginary parts (2.40) and (2.8) have the associated subtraction terms. These masses and widths can also be defined in terms of the more conventional pole masses and widths [37, denoted by $m_{V, p}$ and $\Gamma_{V, p}$, as follows 38]:

$$
\begin{aligned}
& m_{V}^{2}=m_{V, p}^{2}+\Gamma_{V, p}^{2}, \\
& \Gamma_{V}=\Gamma_{V, p} \sqrt{1+\left(\Gamma_{V, p} / m_{V, p}\right)^{2}} .
\end{aligned}
$$

The Breit-Wigner propagator function with the fixed width and that with the running width are then related by the exact relation 38

$$
\frac{1}{s-m_{V, p}^{2}+i m_{V, p} \Gamma_{V, p}}=\frac{1+i \Gamma_{V} / m_{V}}{s-m_{V}^{2}+i s \Gamma_{V} / m_{V}} .
$$

The imaginary part of the numerator $\Delta_{Z Z}\left(q^{2}\right)(2.4 q)$ and $\Delta_{W W}\left(q^{2}\right)(2.8)$ are arranged such that the imaginary parts of the full amplitudes vanish exactly at zero momentum transfer: $\Delta_{V V}(0)=0$. The theta function $\theta(s)(\theta(s)=1$ for $s>0$ and $\theta(s)=0$ for $s \leq 0)$ in the running width factor of eqs. (2.2) and (2.6) then ensures the reality of the amplitudes at $s<0$. It should be noted that the imaginary part $\Delta_{V V}\left(q^{2}\right)$ vanishes at $q^{2}=m_{V}^{2}$ at one-loop level, if all the contributing particle masses can be neglected. As long 
as the relations (2.14) and (2.15) are respected, physical consequences for observables near the $W$ - or $Z$-poles remain unchanged. When constraining the electroweak parameters, however, we often refer to the weak currents at zero momentum transfer. The masses in the LEP convention are more appropriate to use in this case [38, since they absorb reducible higher order contributions from the $W$ and $Z$ widths.

\subsection{Vertex and box corrections}

In this subsection, the vertex and box corrections are numerically estimated in the $\mathrm{SM}$, while their explicit forms are given in appendix A. First the neutral current $(N C)$ amplitudes near the $Z$-pole and at low energies $\left(\left|q^{2}\right| \ll m_{Z}^{2}\right)$ are discussed, then the charged current $(C C)$ amplitudes in the zero momentum transfer limit. Except for the $Z b_{L} b_{L}$ vertex, all the vertex and box corrections are assumed to be dominated by these SM contributions in the following analysis.

Four types of vertex form factors appear in the $N C$ amplitudes (2.2). $\Gamma_{1}^{f}$ and $\bar{\Gamma}_{2}^{f}$ appear both in the $\gamma f f$ and $Z f f$ vertices, while $\Gamma_{3}^{f}$ and $\Gamma_{4}^{f}$ appear only in the $Z f f$ vertices:

$$
\begin{aligned}
& \Gamma^{\gamma f f}\left(q^{2}\right)=-\hat{e}\left\{Q_{f}\left[1+\Gamma_{1}^{f}\left(q^{2}\right)\right]+I_{3 f} \bar{\Gamma}_{2}^{f}\left(q^{2}\right)\right\} \\
& \Gamma^{Z f f}\left(q^{2}\right)=-\hat{g}_{Z}\left\{\left(I_{3 f}-Q_{f} \hat{s}^{2}\right)\left[1+\Gamma_{1}^{f}\left(q^{2}\right)\right]+I_{3 f}\left[\hat{c}^{2} \bar{\Gamma}_{2}^{f}\left(q^{2}\right)+\Gamma_{3}^{f}\left(q^{2}\right)\right]+\Gamma_{4}^{f}\left(q^{2}\right)\right\}
\end{aligned}
$$

The SM contribution to the vertex form factors that are non-vanishing at one-loop order are $\Gamma_{1}^{f_{\alpha}}\left(q^{2}\right), \bar{\Gamma}_{2}^{f_{L}}\left(q^{2}\right)$ and $\Gamma_{3}^{f_{L}}\left(q^{2}\right)$. They can be expressed by

$$
\begin{aligned}
& \Gamma_{1}^{f_{R}}\left(q^{2}\right)=\left(\frac{g_{R}^{Z f f}}{4 \pi}\right)^{2} \Gamma_{1 Z}^{f}\left(q^{2}\right), \\
& \Gamma_{1}^{f_{L}}\left(q^{2}\right)=\left(\frac{g_{L}^{Z f f}}{4 \pi}\right)^{2} \Gamma_{1 Z}^{f}\left(q^{2}\right)+\sum_{f^{\prime}}\left|\frac{g_{L}^{W f f^{\prime}}}{4 \pi}\right|^{2} \Gamma_{1 W}^{f^{\prime}}\left(q^{2}\right), \\
& \bar{\Gamma}_{2}^{f_{L}}\left(q^{2}\right)=-2 \sum_{f^{\prime}}\left|\frac{g_{L}^{W f f^{\prime}}}{4 \pi}\right|^{2} \bar{\Gamma}_{2 W}^{f^{\prime}}\left(q^{2}\right), \\
& \Gamma_{3}^{f_{L}}\left(q^{2}\right)=\sum_{f^{\prime}}\left|\frac{g_{L}^{W f f^{\prime}}}{4 \pi}\right|^{2} \Gamma_{m W}^{f^{\prime}}\left(q^{2}\right),
\end{aligned}
$$

with the gauge-boson-fermion coupling convention

$$
\begin{array}{cc}
g_{L}^{\gamma f f}=g_{R}^{\gamma f f}=\hat{e} Q_{f}, & g_{R}^{Z f f}=\hat{g}_{Z}\left(-Q_{f} \hat{s}^{2}\right), \\
g_{L}^{Z f f}=\hat{g}_{Z}\left(I_{3 f}-Q_{f} \hat{s}^{2}\right), & g_{L}^{W f f^{\prime}}=\frac{\hat{g}}{\sqrt{2}} V_{f f^{\prime}} .
\end{array}
$$


Explicit forms of the functions $\Gamma_{1 V}^{f}\left(q^{2}\right), \bar{\Gamma}_{2 W}^{f^{\prime}}\left(q^{2}\right)$ and $\Gamma_{m W}^{f^{\prime}}\left(q^{2}\right)$ are given in eqs. A.18)(A.20) in appendix A.2. External fermion self-energy corrections are included in the functions $\Gamma_{1 Z}^{f}\left(q^{2}\right)$ and $\Gamma_{1 W}^{f^{\prime}}\left(q^{2}\right)$. For right-handed fermions $\bar{\Gamma}_{2}^{f_{R}}\left(q^{2}\right)=0$ holds, since only those diagrams with $W$ exchange contribute to the vertex function $\bar{\Gamma}_{2}$ at one-loop order. The vertex functions $\Gamma_{3}^{f_{\alpha}}(s)$ are found to be proportional to the square of the fermion mass inside the loop, and are non-vanishing only for $f_{\alpha}=b_{L}$ in the SM, within our approximation of using diagonal KM matrix elements and neglecting terms of order $\left(m_{b} / m_{Z}\right)^{2} \alpha$. For large $m_{t}\left(m_{t}^{2} \gg|s|\right)$, the SM contribution to $\Gamma_{3}^{b_{L}}(s)$ is proportional to $m_{t}^{2} / m_{W}^{2}$ 39, 40]. The functions $\Gamma_{4}^{f_{\alpha}}(s)$ can, in general, be present, but happen to vanish for all $f_{\alpha}$ in the SM; they are, however, found to be non-vanishing in some extended models such as the minimal SUSY-SM. These analytic expressions agree with the known results of refs. 40 44][.

The numerical values of the vertex form factors $\Gamma_{1}^{f}\left(q^{2}\right), \bar{\Gamma}_{2}^{f}\left(q^{2}\right)$ and $\Gamma_{3}^{f}\left(q^{2}\right)$ at $q^{2}=m_{Z}^{2}$ are given in Table 3. All the numerical results presented in this section and in the following sections are obtained by setting

$$
\begin{aligned}
4 \pi / \hat{e}^{2} & =128.72, \\
\hat{s}^{2} & =0.2312,
\end{aligned}
$$

with $\hat{e}=\hat{g} \hat{s}=\hat{g}_{Z} \hat{s} \hat{c}$ in the one-loop correction terms. They are fixed by using the renormalization conditions (2.13) and the SM predictions for $\bar{\alpha}\left(m_{Z}^{2}\right)$ and $\bar{s}^{2}\left(m_{Z}^{2}\right)$ at $m_{t}=$ $150 \mathrm{GeV}, m_{H}=100 \mathrm{GeV}, \alpha_{s}\left(m_{Z}\right)=0.120$ and $\delta_{\alpha}=0$. We emphasize that we do not change the numerical values of eq. (2.19) when discussing experimental constraints on the charge form factors $\bar{e}^{2}\left(m_{Z}^{2}\right)$ and $\bar{s}^{2}\left(m_{Z}^{2}\right)$. All our predictions for the $Z$ parameters can be reproduced simply by using the numerical values listed in Table 3 and eq. (2.19), together with the imaginary parts of the gauge boson propagator corrections

\begin{tabular}{|l|cccc|}
\hline$\alpha_{s}\left(m_{Z}\right)_{\overline{\mathrm{MS}}}$ & 0 & 0.11 & 0.12 & 0.13 \\
\hline$\Delta_{\gamma \gamma}\left(m_{Z}^{2}\right)$ & 0.01726 & 0.01760 & 0.01763 & 0.01766 \\
$\Delta_{\gamma Z}\left(m_{Z}^{2}\right)$ & 0.00248 & 0.00257 & 0.00257 & 0.00258 \\
$\Delta_{Z Z}\left(m_{Z}^{2}\right)$ & 0.00005 & 0.00003 & 0.00003 & 0.00003 \\
\hline
\end{tabular}

which are obtained by using the perturbative order $\alpha \alpha_{s}$ approximations of appendix A with the effective quark masses of eqs. (B.25) and (B.26). It is worth noting that the

${ }^{2}$ We note the following misprints in ref. [40]. In the last line of eq. (2.7), the factor $1 /\left(m^{2}-M^{2}\right)^{2}$ should read $1 /\left(m^{2}-M^{2}\right)$. In the first line of eq. (2.8), the term $4 q^{4} M^{2}$ should read $4 q^{2} M^{4}$, and in the last line of eq. (2.9), the term $m / 2 M^{2}$ should read $m^{2} / 2 M^{2}$. Our vertex functions $\Gamma_{1 W}^{f}, \bar{\Gamma}_{2 W}^{f^{\prime}}$ and $\Gamma_{m W}^{f^{\prime}}$ are then related to their functions $\rho, \Lambda$ and $\Xi$ by the identities : $\Gamma_{1 W}^{f^{\prime}}\left(q^{2}\right)=\rho\left(-q^{2}, m_{W}^{2}, m_{f^{\prime}}^{2}\right), \bar{\Gamma}_{2 W}^{f^{\prime}}\left(q^{2}\right)=[\rho+\Lambda]\left(-q^{2}, m_{W}^{2}, m_{f^{\prime}}^{2}\right)+$ $2\left[B_{0}\left(q^{2} ; m_{W}, m_{W}\right)-B_{0}\left(0 ; m_{W}, m_{W}\right)\right]$, and $\Gamma_{m W}^{f^{\prime}}\left(q^{2}\right)=-2 \Xi\left(-q^{2}, m_{W}^{2}, m_{f^{\prime}}^{2}\right)$. 
real part of the vertex corrections (Table 3) and the imaginary part $\Delta_{\gamma \gamma}\left(m_{Z}^{2}\right)$ interfere with the leading $Z$-pole amplitude: the latter contribution has been subtracted in the $Z$ parameters [26], whereas the former contributions modify the scattering amplitudes by as much as $0.5 \%$, and hence they can contribute to the cross sections at the $1 \%$ level.

Note further that the vertex correction without the pinch term subtraction [3, 41] $\Gamma_{2}^{f_{L}}\left(q^{2}\right)$ is related to the $\bar{\Gamma}_{2}^{f_{L}}\left(q^{2}\right)$ function by

$$
\bar{\Gamma}_{2}^{f_{L}}\left(q^{2}\right)=\Gamma_{2}^{f_{L}}\left(q^{2}\right)-\frac{\hat{g}^{2}}{8 \pi^{2}} \operatorname{Re}\left[B_{0}\left(q^{2} ; W, W\right)-B_{0}(0 ; W, W)\right]
$$

in the t' Hooft-Feynman gauge. The difference is universal ( $f$-independent) and we find $\bar{\Gamma}_{2}^{f_{L}}\left(m_{Z}^{2}\right)=\Gamma_{2}^{f_{L}}\left(m_{Z}^{2}\right)-0.00134$. The vertex corrections are slightly larger in magnitude after subtraction of the pinch term.

It is convenient to introduce the following special form factor

$$
\bar{\delta}_{b}(s)=\Gamma_{1}^{b_{L}}(s)+\hat{c}^{2} \bar{\Gamma}_{2}^{b_{L}}(s)+\Gamma_{3}^{b_{L}}(s)+\text { higher order terms },
$$

which is treated also as a free parameter in our fit at $s=m_{Z}^{2}$ to deal with the strong $m_{t}$ dependence of the $Z b_{L} b_{L}$ vertex (see also ref. [12, 14]). In this way, the importance of the $Z b_{L} b_{L}$ vertex correction [45] can be assessed independently of the specific SM mechanism and also the data analysis is kept separate from the evaluation of $\bar{\delta}_{b}$ in a specific model. In the SM, the parameter $\bar{\delta}_{b}$ can be evaluated by including $O\left(\alpha_{s} m_{t}^{2}\right)$ 46] and $O\left(m_{t}^{4}\right)$ 47, 48] two-loop corrections of the SM, which are given explicitly in appendix C.4: see eq. (C.54).

At low energies, light fermion masses may not be neglected compared to the momentum transfer $q^{2}$. In the limit of $\left|q^{2}\right| / m_{Z}^{2} \ll 1$ and $m_{f}^{2} / m_{Z}^{2} \ll 1$ but at fixed $m_{f}^{2} / q^{2}$, the vertex functions reduce to

$$
\begin{aligned}
\Gamma_{1 Z}^{f}\left(q^{2}\right) & =\frac{-q^{2}}{m_{Z}^{2}}\left[J_{Z}\left(q^{2} ; f\right)+O\left(\frac{q^{2}}{m_{Z}^{2}}\right)\right], \\
\Gamma_{1 W}^{f^{\prime}}\left(q^{2}\right) & =\frac{-q^{2}}{m_{W}^{2}}\left[J_{W}\left(q^{2} ; f^{\prime}\right)+O\left(\frac{q^{2}}{m_{Z}^{2}}\right)\right], \\
\bar{\Gamma}_{2 W}^{f^{\prime}}\left(q^{2}\right) & =\frac{-q^{2}}{m_{W}^{2}}\left[\bar{J}_{W}\left(q^{2} ; f^{\prime}\right)+O\left(\frac{q^{2}}{m_{Z}^{2}}\right)\right] .
\end{aligned}
$$

The functions $J_{Z}\left(q^{2} ; f\right), J_{W}\left(q^{2} ; f\right)$ and $\bar{J}_{W}\left(q^{2} ; f\right)$ have the same form as the fermionic contribution to the neutral gauge boson vacuum polarization functions: see eq. (A.27). The form factor $\bar{\Gamma}_{2}^{\nu_{L}}\left(q^{2}\right)$ is often called the neutrino charge radius term 49. The subtraction of the pinch term makes it gauge invariant 34.

For the $N C$ process $f_{\alpha}\left(p_{1}\right) f_{\beta}^{\prime}\left(p_{2}\right) \rightarrow f_{\alpha}\left(p_{3}\right) f_{\beta}^{\prime}\left(p_{4}\right)$, as well as for its crossed channels, the box correction terms in eq. (2.2) can be expressed as

$$
B_{\alpha, \alpha}^{f f^{\prime}}(s, t)=\frac{1}{16 \pi^{2}}\left|g_{\alpha}^{Z f f} g_{\alpha}^{Z f^{\prime} f^{\prime}}\right|^{2}\left[I_{1}\left(u, s ; m_{Z}, 0\right)-I_{2}\left(t, s ; m_{Z}, 0\right)\right]
$$




$$
\begin{gathered}
+\frac{\delta_{\alpha L}}{16 \pi^{2}}\left|g_{L}^{W f f^{\prime \prime}} g_{L}^{W f^{\prime} f^{\prime \prime \prime}}\right|^{2} \times\left\{\begin{array}{l}
+I_{1}\left(u, s ; m_{W}, m_{f^{\prime \prime \prime}}\right) \\
-I_{2}\left(t, s ; m_{W}, m_{f^{\prime \prime \prime}}\right)
\end{array} \text { for } I_{3 f} I_{3 f^{\prime}}<0\right. \\
B_{\alpha,-\alpha}^{e f}(s, t)=\frac{1}{16 \pi^{2}}\left|g_{\alpha}^{Z f f} g_{-\alpha}^{Z f^{\prime} f^{\prime}}\right|^{2}\left[I_{2}\left(u, s ; m_{Z}, 0\right)-I_{1}\left(t, s ; m_{Z}, 0\right)\right],
\end{gathered}
$$

where $s=\left(p_{1}-p_{3}\right)^{2}, t=\left(p_{1}-p_{4}\right)^{2}$ and $u=\left(p_{1}+p_{2}\right)^{2}$ are the Mandelstam variables satisfying $s+t+u=0$. In the second term of (2.24b), $f^{\prime \prime}$ and $f^{\prime \prime \prime}$ are the weak isospin partners of $f$ and $f^{\prime}$, respectively, where all external and internal fermion masses except for $m_{f^{\prime \prime \prime}}$ are neglected : the upper term $\left(I_{3 f} I_{3 f^{\prime}}<0\right)$ should be taken for $\left(f, f^{\prime}\right)=(\ell, u)$, $(\nu, \ell)$, and $(\nu, d)$, whereas the lower term $\left(I_{3 f} I_{3 f^{\prime}}>0\right)$ for $\left(f, f^{\prime}\right)=(\ell, d)$, and $(\nu, u)$. The explicit form of the box functions $I_{1}$ and $I_{2}$ are given in eq. (A.30) of appendix A.3. These analytic expressions agree with the known results of refs. 40 44. It is worth noting here that the box contributions to the helicity amplitudes have the above simple current product form only when the external fermion masses can be neglected.

The numerical values of the box functions $B_{i j}(s, t)$ for the process $e^{+} e^{-} \rightarrow f \bar{f}$ are given in Table 4 for $s=-2 t=m_{Z}^{2}$. They contribute negligibly to the $Z$ parameters, because they do not interfere with the dominant $Z$-pole amplitudes being almost purely imaginary near the pole. The imaginary parts appear in the box functions only above the $W$-pair production threshold.

The box contributions are found to be non-negligible in some low energy $N C$ processes. In the $s=t=u=0$ limit, one finds

$$
\begin{aligned}
& I_{1}\left(0,0 ; m_{V}, 0\right)=-\frac{4}{m_{V}^{2}} \\
& I_{2}\left(0,0 ; m_{V}, 0\right)=-\frac{1}{m_{V}^{2}} .
\end{aligned}
$$

The $W W$ box contributions to the processes with the $I_{1}$ function, that is, the low energy $\nu-\ell, \nu-d$ and $e-u$ scattering processes are found to be significant.

Precise values of the charged current matrix elements are needed only at low energies. The muon decay constant is given by

$$
G_{F}=\frac{\bar{g}_{W}^{2}(0)+\hat{g}^{2} \bar{\delta}_{G}}{4 \sqrt{2} m_{W}^{2}},
$$

where the factor $\bar{\delta}_{G}$

$$
\bar{\delta}_{G}=\frac{\hat{g}^{2}}{8 \pi^{2}}\left[1+\left(\frac{1}{4 \hat{s}^{2}}-1\right) \ln \frac{1}{\hat{c}^{2}}\right] \approx 0.0055
$$

denotes the sum of the vertex and the box contributions in the SM. Its numerical value above is obtained for the couplings of eq. (2.19). The identity (2.26) gives the physical $W$ 
mass in terms of $\bar{g}_{W}^{2}(0)$, once the $\bar{\delta}_{G}$ value is known for a given model. The overline here again indicates the removal of the pinch terms with the consequence that the numerical value is significantly (about 20\%) smaller than the standard factor 50

$$
\delta_{G}=\frac{\hat{g}^{2}}{8 \pi^{2}}\left[3-\left(\frac{7}{4 \hat{s}^{2}}-1\right) \ln \frac{1}{\hat{c}^{2}}\right] \approx 0.0068
$$

which was obtained simply by subtracting the singular vertex function at zero momentum transfer. The difference

$$
\bar{\delta}_{G}-\delta_{G}=\frac{\hat{g}^{2}}{4 \pi^{2}}\left[B_{0}(0 ; W, W)-\hat{c}^{2} B_{0}(0 ; W, Z)-\hat{s}^{2} B_{0}(0 ; W, \gamma)\right]
$$

is the pinch term contribution [34]. Note that the sum of the propagator and the vertex/box corrections is scheme-independent and that the correction term $\bar{\delta}_{G}$ of eq. (2.27) should be used together with the charge form factor $\bar{g}_{W}^{2}(0)$ which contains the associated pinch term.

\subsection{Constraints due to $\alpha, G_{F}$ and $m_{Z}$}

Among the electroweak observables the three quantities $\alpha, G_{F}$ and $m_{Z}$ have been measured with outstanding precision, namely $\Delta \alpha / \alpha \approx 5 \times 10^{-8}, \Delta G_{F} / G_{F} \approx 2 \times 10^{-5}$ [25], and $\Delta m_{Z} / m_{Z} \approx 8 \times 10^{-5}$ [26]. For this reason $\alpha, G_{F}$ and $m_{Z}$ are chosen as our basic electroweak parameters and treated as constants in the analysis (see Tables 1 and 2).

On the other hand, the tree-level properties of the gauge boson propagators are fixed completely by three parameters, the two gauge couplings $g$ and $g^{\prime}$ for the $\mathrm{SU}(2)_{\mathrm{L}}$ and $\mathrm{U}(1)_{\mathrm{Y}}$ gauge groups, respectively, and one vacuum expectation value $v \equiv\left(\sqrt{2} G_{F}\right)^{-1 / 2} \approx$ $246 \mathrm{GeV}$, in models where the electroweak symmetry breaking sector has the custodial $\mathrm{SU}(2)$ symmetry [51]. Consequently, the four charge form factors $\bar{e}^{2}\left(q^{2}\right), \bar{s}^{2}\left(q^{2}\right), \bar{g}_{Z}^{2}\left(q^{2}\right)$, and $\bar{g}_{W}^{2}\left(q^{2}\right)$ are completely determined by finite quantum corrections in this class of models when expressed in terms of the three constants $\alpha, G_{F}$ and $m_{Z}$.

In this subsection, the prescription for calculating all charge form factors in terms of $\left(\alpha, G_{F}, m_{Z}\right)$ is given explicitly in an arbitrary model with the broken $\mathrm{SU}(2)_{\mathrm{L}} \times \mathrm{U}(1)_{\mathrm{Y}}$ gauge symmetry. Their numerical predictions are given in the SM.

The form factor $\bar{e}^{2}\left(q^{2}\right) \equiv 4 \pi \bar{\alpha}\left(q^{2}\right)$ is fixed by the following identity

$$
\frac{1}{\bar{\alpha}\left(q^{2}\right)}-\frac{1}{\alpha}=4 \pi \operatorname{Re}\left[\bar{\Pi}_{T, \gamma}^{Q Q}\left(q^{2}\right)-\bar{\Pi}_{T, \gamma}^{Q Q}(0)\right],
$$

which gives the renormalization group improved running $\bar{\alpha}\left(q^{2}\right)$ as explained in appendix B. Here $\bar{\Pi}_{T}^{Q Q}\left(q^{2}\right)$ is the $\gamma \gamma$ propagator function without the overall coupling factor $\hat{e}^{2}$ [2]: see eq. (A.1). 
In principle, the effective coupling $\bar{\alpha}\left(m_{Z}^{2}\right)$ can be calculated from the observed $\alpha$ value by using the above identity. In practice, however, the right-hand side suffers from nonperturbative QCD corrections to the light quark contributions. We make use of the dispersion analyses [27 29] to estimate the hadronic contributions to the running of $\bar{\alpha}\left(q^{2}\right)$ and $\bar{s}^{2}\left(q^{2}\right)$ form factors at $0<\left|q^{2}\right|<m_{Z}^{2}$. Details can be found in appendix B.

In order to take account of uncertainty in the hadronic contribution and also possible new physics contributions, the parameter $\delta_{\alpha}$ is introduced as an external parameter in the analysis :

$$
\frac{1}{\bar{\alpha}\left(m_{Z}^{2}\right)} \equiv 128.72+\delta_{\alpha}
$$

which can be expressed by

$$
\delta_{\alpha} \approx \delta_{\mathrm{had}}+4 \pi \operatorname{Re}\left[\bar{\Pi}_{T, \gamma}^{Q Q}\left(m_{Z}^{2}\right)-\bar{\Pi}_{T, \gamma}^{Q Q}(0)\right]_{\text {NewPhysics }},
$$

for $m_{t}=150-200 \mathrm{GeV}$ as stated in eqs. (B.32) and (B.30) of Appendix B. Here $\delta_{\text {had }}=$ $0 \pm 0.10(\mathbb{B} .22)$ is the present estimate [28] for the hadronic contribution. The parameter $\delta_{\alpha}$ being treated as an external parameter serves also to assess future improvements in low energy $e^{+} e^{-}$hadroproduction experiments as well as possible new physics contributions.

The remaining three charge form factors can be fixed by introducing the three radiative parameters $S, T$ and $U$ that are defined by the following identities:

$$
\begin{aligned}
& \frac{\bar{g}_{W}^{2}(0)}{m_{W}^{2}} \frac{m_{Z}^{2}}{\bar{g}_{Z}^{2}(0)} \equiv 1-\alpha T, \\
& \frac{4 \pi}{\bar{g}_{Z}^{2}(0)}-\frac{\bar{s}^{2}\left(m_{Z}^{2}\right) \bar{c}^{2}\left(m_{Z}^{2}\right)}{\bar{\alpha}\left(m_{Z}^{2}\right)} \equiv-\frac{S}{4}, \\
& \frac{4 \pi}{\bar{g}_{W}^{2}(0)}-\frac{\bar{s}^{2}\left(m_{Z}^{2}\right)}{\bar{\alpha}\left(m_{Z}^{2}\right)} \equiv-\frac{S+U}{4} .
\end{aligned}
$$

The parameters $S, T$ and $U$ can be calculated perturbatively in any models from the gauge boson propagator functions of eq. (A.1) by

$$
\begin{aligned}
S & =16 \pi \operatorname{Re}\left[\bar{\Pi}_{T, \gamma}^{3 Q}\left(m_{Z}^{2}\right)-\bar{\Pi}_{T, Z}^{33}(0)\right], \\
T & =\frac{4 \sqrt{2} G_{F}}{\alpha} \operatorname{Re}\left[\bar{\Pi}_{T}^{33}(0)-\bar{\Pi}_{T}^{11}(0)\right], \\
U & =16 \pi \operatorname{Re}\left[\bar{\Pi}_{T, Z}^{33}(0)-\bar{\Pi}_{T, W}^{11}(0)\right] .
\end{aligned}
$$

For models without custodial SU(2) symmetry, the $T$ parameter is sensitive to the ultraviolet cut-off, and hence is un-calculable from $\left(\alpha, G_{F}, m_{Z}\right)$ alone. In this case it should be regarded as the fourth basic parameter of the theory. 
Our definitions (2.33) of the three parameters $S, T, U$ are inspired by the pioneering work of Peskin and Takeuchi 田. Our definition, in contrast to theirs, includes all radiative effects from both SM and new physics contributions. The original parameters, denoted below by the index PT, are approximately related to ours by subtracting the SM contributions evaluated at $m_{t}=150 \mathrm{GeV}$ and $m_{H}=1000 \mathrm{GeV}$ :

$$
\begin{aligned}
& S_{\mathrm{PT}} \approx S-S_{\mathrm{SM}}\left(m_{t}=150 \mathrm{GeV}, m_{H}=1000 \mathrm{GeV}\right), \\
& T_{\mathrm{PT}} \approx T-T_{\mathrm{SM}}\left(m_{t}=150 \mathrm{GeV}, m_{H}=1000 \mathrm{GeV}\right), \\
& U_{\mathrm{PT}} \approx U-U_{\mathrm{SM}}\left(m_{t}=150 \mathrm{GeV}, m_{H}=1000 \mathrm{GeV}\right),
\end{aligned}
$$

provided the scale of new physics is much larger than $m_{Z}$. The expressions (2.34) agree with the modified $S, T, U$ parameters of ref. [34]. The same form of the definitions without the pinch terms (in the 't Hooft Feynman gauge) have been used in some earlier works [11, 52, 53].

Explicit forms of the SM contributions to the $S, T, U$ parameters are given in appendix $\mathrm{C}$, together with the SM contribution to the $Z b_{L} b_{L}$ form factor $\bar{\delta}_{b}\left(m_{Z}^{2}\right)$. All the known two-loop corrections of order $\alpha \alpha_{s}$ [46, 54 56] and order $m_{t}^{4}$ [47, 48, 57, 58] are included. The recently found [56, 59] small two-loop corrections of order $m_{H}^{2}$ are neglected. For practical reasons we adopt the perturbative order $\alpha \alpha_{s}$ [46, 54 56] corrections at $\alpha_{s}=\alpha_{s}\left(m_{Z}\right)_{\overline{\mathrm{MS}}}$ in calculating all the parameters $S, T, U$ and $\bar{\delta}_{b}\left(m_{Z}^{2}\right)$. The reader can therefore unambiguously reproduce our results. The effects due to non-perturbative threshold corrections [60 62] should be evaluated separately, and one can obtain more precise predictions of the SM from our formulae by adjusting the effective top-quark mass to produce the same $S, T, U$, and $\bar{\delta}_{b}\left(m_{Z}^{2}\right)$ values. It should be noted that at present the uncertainty in the SM contribution to the $T$ parameter is such that $m_{t}$ can be predicted with a few $\mathrm{GeV}$ uncertainty for a given $T$ value [62]. Fig. [1 shows the SM contributions to the $S, T, U$ and $\bar{\delta}_{b}\left(m_{Z}^{2}\right)$ parameters as functions of $m_{t}$ for $m_{H}=1-1000 \mathrm{GeV}$ at $\alpha_{s}\left(m_{Z}\right)=0.12$. It is worth noting that the $T$ and $\bar{\delta}_{b}\left(m_{Z}^{2}\right)$ parameters are proportional to $m_{t}^{2}$ for large $m_{t}\left(m_{t}^{2} \gg m_{Z}^{2}\right)$, the parameters $U$ and $\bar{\delta}_{b}\left(m_{Z}^{2}\right)$ are almost independent of $m_{H}$, the $T$ parameter decreases with increasing $m_{H}$, and the $S$ parameter becomes negative for small $m_{H}$.

Once the $S, T, U$ parameters are calculated in a given model, the three charge form factors can be predicted as follows :

$$
\begin{aligned}
\frac{1}{\bar{g}_{Z}^{2}(0)} & =\frac{1+\bar{\delta}_{G}-\alpha T}{4 \sqrt{2} G_{F} m_{Z}^{2}}, \\
\bar{s}^{2}\left(m_{Z}^{2}\right) & =\frac{1}{2}-\sqrt{\frac{1}{4}-\bar{e}^{2}\left(m_{Z}^{2}\right)\left(\frac{1}{\bar{g}_{Z}^{2}(0)}+\frac{S}{16 \pi}\right)},
\end{aligned}
$$




$$
\frac{1}{\bar{g}_{W}^{2}(0)}=\frac{\bar{s}^{2}\left(m_{Z}^{2}\right)}{\bar{e}^{2}\left(m_{Z}^{2}\right)}-\frac{1}{16 \pi}(S+U) .
$$

The expression (2.36a) follows from eqs. (2.33a) and (2.26) up to terms of order $\alpha^{2}$. Its explicit form takes account of the reducible order $m_{t}^{4}$ corrections [63], and it makes clear that the combination

$$
\bar{\delta}_{G}-\alpha T
$$

determines the neutral current charge form factor $\bar{g}_{Z}^{2}\left(q^{2}\right)$ in terms of $G_{F} m_{Z}^{2}$. In fact, the pinch term contribution to $T$ in eq. (2.34b) and the one removed from the vertex contribution in $\bar{\delta}_{G}(2.27)$ cancel in the combination $\bar{\delta}_{G}-\alpha T$.

It is clear from eqs. (2.36) that $\bar{g}_{Z}^{2}(0)$ is fixed by $\bar{\delta}_{G}-\alpha T, \bar{s}^{2}\left(m_{Z}^{2}\right)$ by $\bar{g}_{Z}^{2}(0), \bar{\alpha}\left(m_{Z}^{2}\right)$ and $S$, and $\bar{g}_{W}^{2}(0)$ by $\bar{s}^{2}\left(m_{Z}^{2}\right), \bar{\alpha}\left(m_{Z}^{2}\right)$ and $S+U$. It is instructive to express these form factors approximately as linear combinations of the parameters $S, T, U$ and $\delta_{\alpha}$ :

$$
\begin{array}{rlr}
\bar{g}_{Z}^{2}(0) & =0.5456 \quad+0.0040 T, \\
\bar{s}^{2}\left(m_{Z}^{2}\right) & =0.2334+0.0036 S-0.0024 T \quad-0.0026 \delta_{\alpha}, \\
\bar{g}_{W}^{2}(0) & =0.4183-0.0030 S+0.0044 T+0.0035 U+0.0014 \delta_{\alpha} .
\end{array}
$$

Expressed in this form, it becomes obvious that essentially $\bar{g}_{Z}^{2}(0)$ measures $T, \bar{s}^{2}\left(m_{Z}^{2}\right)$ measures $S-0.7 T$, and $\bar{g}_{W}^{2}(0)$ measures $T+0.8 U-0.7 S$, if the $\mathrm{SM}$ values of $\bar{\delta}_{G}$ and $\delta_{\alpha}$ are assumed. Here the coefficients are obtained by setting $\bar{\delta}_{G}=0.0055$. Results for arbitrary $\bar{\delta}_{G}$ are obtained by the replacement :

$$
T \rightarrow T+\frac{0.0055-\bar{\delta}_{G}}{\alpha}
$$

Note that the combination $\bar{\delta}_{G}-\alpha T$ vanishes in the $\operatorname{SM}\left(\bar{\delta}_{G} \approx 0.0055\right)$ for $T \approx 0.75$. Fig. 1 shows that this cancellation occurs at around $m_{t} \approx 175 \mathrm{GeV}$. The SM predictions for the neutral current experiments can then be reproduced rather accurately by using the 'tree-level' predictions with $\bar{\delta}_{G}-\alpha T=S=0$ in eqs. (2.36), since the SM contribution to $S$ is rather small. This should not, however, be interpreted as absence of any quantum corrections 64 (that is, $\bar{\delta}_{G}=T=0$ ), but rather as evidence for the large quantum correction $\alpha T \approx 0.0055$ within the $\mathrm{SM}$ (see also section 5.3).

Finally, the running of the remaining three charge form factors are calculated by

$$
\begin{aligned}
& \frac{\bar{s}^{2}\left(q^{2}\right)}{\bar{e}^{2}\left(q^{2}\right)}-\frac{\bar{s}^{2}\left(m_{Z}^{2}\right)}{\bar{e}^{2}\left(m_{Z}^{2}\right)}=\operatorname{Re}\left[\bar{\Pi}_{T, \gamma}^{3 Q}\left(q^{2}\right)-\bar{\Pi}_{T, \gamma}^{3 Q}\left(m_{Z}^{2}\right)\right] \\
& \frac{1}{\bar{g}_{Z}^{2}\left(q^{2}\right)}-\frac{1}{\bar{g}_{Z}^{2}(0)}=\operatorname{Re}\left[\bar{\Pi}_{T, Z}^{33}\left(q^{2}\right)-\bar{\Pi}_{T, Z}^{33}(0)\right]-2 \hat{s}^{2} \operatorname{Re}\left[\bar{\Pi}_{T, Z}^{3 Q}\left(q^{2}\right)-\bar{\Pi}_{T, Z}^{3 Q}(0)\right]
\end{aligned}
$$




$$
\begin{aligned}
& +\hat{s}^{4} \operatorname{Re}\left[\bar{\Pi}_{T, Z}^{Q Q}\left(q^{2}\right)-\bar{\Pi}_{T, Z}^{Q Q}(0)\right], \\
\frac{1}{\bar{g}_{W}^{2}\left(q^{2}\right)}-\frac{1}{\bar{g}_{W}^{2}(0)}= & \operatorname{Re}\left[\bar{\Pi}_{T, W}^{11}\left(q^{2}\right)-\bar{\Pi}_{T, W}^{11}(0)\right] .
\end{aligned}
$$

Equation (2.40a) is the solution of the RG equation (see appendix B), and hence is valid at arbitrary $q^{2}$. At $\left|q^{2}\right|<m_{Z}^{2}$, the parametrizations of the dispersive analysis [27 29] are used for the light quark contribution. Equations (2.40b) and (2.400) are valid perturbative expressions provided $\left|q^{2}\right| \lesssim O\left(m_{Z}^{2}\right)$. At very high energies $\left(\left|q^{2}\right| \gg m_{Z}^{2}\right)$, the more elaborate expressions (B.38)-(B.41) should be used to estimate accurately the charge form factors $\bar{g}_{Z}^{2}\left(q^{2}\right)$ and $\bar{g}_{W}^{2}\left(q^{2}\right)$.

Fig. 2 displays the four charge form factors $1 / \bar{\alpha}\left(q^{2}\right), \bar{s}^{2}\left(q^{2}\right), \bar{g}_{Z}^{2}\left(q^{2}\right)$ and $\bar{g}_{W}^{2}\left(q^{2}\right)$ as functions of $\sqrt{\left|q^{2}\right|}$ for both time-like $\left(q^{2}>0\right)$ and space-like $\left(q^{2}<0\right)$ momenta. They are obtained in the SM for several $m_{t}$ and $m_{H}$ values, namely $m_{t}=100,150,200 \mathrm{GeV}$, and $m_{H}=100,1000 \mathrm{GeV}$. The trajectories are fixed such that the known values of the three basic parameters $\left(\alpha, G_{F}\right.$, and $\left.m_{Z}\right)$ are reproduced for $\bar{\delta}_{G}=0.0055, \delta_{\alpha}=0$ and $\alpha_{s}\left(m_{Z}\right)=0.12$. The running of the form factors $\bar{\alpha}\left(q^{2}\right)$ and $\bar{s}^{2}\left(q^{2}\right)$ at $\left|q^{2}\right| \ll m_{Z}^{2}$ is due to the $\mathrm{QED} \times \mathrm{QCD}$ quantum effects [36], and its detailed treatment is given in appendix B. The threshold effects are clearly seen in the time-like trajectories. Light hadron threshold effects do not show up since we adopt the dispersion integral fit of the hadronic contributions to the vacuum polarizations in the space-like region [27 29] also for their contribution in the time-like region. The running of the $\bar{g}_{Z}^{2}\left(q^{2}\right)$ and $\bar{g}_{W}^{2}\left(q^{2}\right)$ form factors freezes at $\left|q^{2}\right| \ll m_{Z}^{2}$. It is clearly seen that the weak boson threshold effects are significant for all the charge form factors in the time-like region[].

In section 4 , the charge form factors (2.3) and (2.7) are determined from the three sectors of the electroweak precision experiments under the assumption that there are no new physics contributions to the vertex and box corrections, except for allowing the $Z b_{L} b_{L}$ vertex to take on arbitrary values.

\section{Predictions of electroweak observables}

In this section, all electroweak observables are expressed in terms of the helicity amplitudes of eqs. (2.2) and (2.6), together with the external QED and QCD correction factors.

${ }^{3}$ Note that the charge form factor $\bar{g}_{W}^{2}\left(q^{2}\right)$ suffers from an infrared singularity at $q^{2}=$ $m_{W}^{2}$ due to the opening of the $W+\gamma$ threshold on the pole [65. The charged current cross section near the $W$-pole may be expressed more conveniently in terms of $\bar{g}_{W}^{2}(0)$, or $G_{F} m_{W}^{2}$. 
The predictions are restricted to the models respecting $\mathrm{SU}(2)_{\mathrm{L}} \times \mathrm{U}(1)_{\mathrm{Y}}$ gauge symmetry with spontaneous breakdown to $\mathrm{U}(1)_{\mathrm{EM}}$ and presented as functions of the charge form factors $\bar{s}^{2}(0), \bar{g}_{Z}^{2}(0), \bar{g}_{W}^{2}(0), \bar{s}^{2}\left(m_{Z}^{2}\right), \bar{g}_{Z}^{2}\left(m_{Z}^{2}\right)$, and the vertex form factor $\bar{\delta}_{b}\left(m_{Z}^{2}\right)$. It is assumed that the remaining vertex and box correction are dominated by the SM contributions.

\section{$3.1 Z$ boson parameters}

The following observables on the $Z$-pole $\left(s=m_{Z}^{2}\right)$ are used in the fit :

$$
\Gamma_{Z}, \sigma_{h}^{0}, R_{\ell}, A_{\mathrm{FB}}^{0, \ell}, P_{\tau}, A_{\mathrm{LR}}, A_{\mathrm{FB}}^{0, b}, A_{\mathrm{FB}}^{0, c}, R_{b}
$$

Since the $Z$ mass $m_{Z}$ is measured very accurately, the value $m_{Z}=91.187 \mathrm{GeV}$ is treated as a constant in the fits. The contributions from the SM box corrections are very small on the $Z$-pole (see Table 4 ), thus the $\cos \theta$-dependence of the box correction factors is neglected.

The total cross section for the process $e^{+} e^{-} \rightarrow f \bar{f}$ is given by

$$
\begin{aligned}
\sigma_{f} \equiv \sigma\left(e^{+} e^{-} \rightarrow f \bar{f}\right) \\
=\frac{s}{48 \pi}\left\{\left(\left|M_{L L}^{e f}+M_{L R}^{e f}\right|^{2}+\left|M_{R L}^{e f}+M_{R R}^{e f}\right|^{2}\right) \frac{C_{f V}}{2}\right. \\
\left.\quad+\left(\left|M_{L L}^{e f}-M_{L R}^{e f}\right|^{2}+\left|M_{R L}^{e f}-M_{R R}^{e f}\right|^{2}\right) \frac{C_{f A}}{2}\right\}\left(1+\frac{3}{4} Q_{f}^{2} \frac{\bar{\alpha}(s)}{\pi}\right),
\end{aligned}
$$

for unpolarized beams, where the last term proportional to $\bar{\alpha}(s) / \pi$ accounts for the final state QED correction. Here and in the following

$$
M_{\alpha \beta}^{e f} \equiv M_{e_{\alpha} f_{\beta}}^{N C}\left(s=\left(p_{e^{-}}+p_{e^{+}}\right)^{2}, t=\left(p_{e^{-}}-p_{f}\right)^{2}\right)
$$

denote the $N C$ amplitudes of eq. (2.2). The factors $C_{q V}$ and $C_{q A}$ for quarks contain the final state QCD corrections for the vector [66] and axial vector current [67,68] contributions, respectively, together with the finite mass corrections of the final state fermions [69]:

$$
\begin{gathered}
C_{q V}=3\left\{\frac{\hat{\beta}_{q}\left(3-\hat{\beta}_{q}^{2}\right)}{2}+a+1.409 a^{2}-12.767 a^{3}\right. \\
\left.+12 \frac{\hat{m}_{q}^{2}(\sqrt{s})}{s}\left(a+8.736 a^{2}+45.146 a^{3}\right)\right\}, \\
C_{q A}=3\left\{\hat{\beta}_{q}^{3}+a+1.409 a^{2}-12.767 a^{3}-6 \frac{\hat{m}_{q}^{2}(\sqrt{s})}{s}\left(\frac{11}{3} a+14.286 a^{2}\right)\right. \\
\left.\mp a^{2}\left[f\left(m_{t}\right)+6 \frac{\hat{m}_{q}^{2}(\sqrt{s})}{s}\left(3+\ln \frac{m_{t}^{2}}{m_{Z}^{2}}\right)\right]\right\},
\end{gathered}
$$


with

$$
\begin{aligned}
a & \equiv a^{(5)}(\sqrt{s}) \equiv \frac{\alpha_{s}(\sqrt{s}) \overline{\mathrm{MS}}}{\pi}, \\
\hat{\beta}_{q} & \equiv \sqrt{1-\frac{4 \hat{m}_{q}^{2}(\sqrt{s})}{s}},
\end{aligned}
$$

where $\hat{m}_{q}(\sqrt{s})$ denotes the $\overline{\mathrm{MS}}$ running quark mass at $\mu=\sqrt{s}$. The masses of the three lightest quarks $(u, d, s)$ are neglected, while the bottom and charm quark running masses, $\hat{m}_{b}(\sqrt{s})$ and $\hat{m}_{c}(\sqrt{s})$, are obtained from $\hat{m}_{b}\left(m_{b}\right)$ and $\hat{m}_{c}\left(m_{c}\right)$ by the two-loop renormalization group equations:

$$
\begin{aligned}
\frac{\hat{m}_{b}(\sqrt{s})}{\hat{m}_{b}\left(m_{b}\right)}= & \frac{\hat{m}_{c}(\sqrt{s})}{\hat{m}_{c}\left(m_{b}\right)}=\left[\frac{a^{(5)}(\sqrt{s})}{a^{(5)}\left(m_{b}\right)}\right]^{\frac{\gamma_{0}^{(5)}}{b_{0}^{(5)}}}\left[\frac{b_{0}^{(5)}+b_{1}^{(5)} a^{(5)}(\sqrt{s})}{b_{0}^{(5)}+b_{1}^{(5)} a^{(5)}\left(m_{b}\right)}\right]\left(\frac{\gamma_{1}^{(5)}}{b_{1}^{(5)}}-\frac{\gamma_{0}^{(5)}}{b_{0}^{(5)}}\right) \\
& \frac{\hat{m}_{c}\left(m_{b}\right)}{\hat{m}_{c}\left(m_{c}\right)}=\left[\frac{a^{(4)}\left(m_{b}\right)}{a^{(4)}\left(m_{c}\right)}\right]^{\gamma_{0}^{(4)}}\left[\frac{b_{0}^{(4)}+b_{1}^{(4)} a^{(4)}\left(m_{b}\right)}{b_{0}^{(4)}+b_{1}^{(4)} a^{(4)}\left(m_{c}\right)}\right]\left(\frac{\gamma_{1}^{(4)}}{b_{1}^{(4)}}-\frac{\gamma_{0}^{(4)}}{b_{0}^{(4)}}\right)
\end{aligned}
$$

where 70,71

$$
\begin{aligned}
& b_{0}^{\left(n_{f}\right)}=\frac{33-2 n_{f}}{6}, \quad b_{1}^{\left(n_{f}\right)}=\frac{153-19 n_{f}}{12}, \\
& \gamma_{0}^{\left(n_{f}\right)}=2, \quad \gamma_{1}^{\left(n_{f}\right)}=\frac{303-10 n_{f}}{36},
\end{aligned}
$$

are the coefficients of the $\beta$-function and the anomalous mass dimension in the effective $n_{f}$-flavor QCD. The running coupling $a^{(4)}(\mu)$ of the effective $n_{f}=4$ theory is calculated from a given $a^{(5)}\left(m_{Z}\right) \equiv \alpha_{s}\left(m_{Z}\right) \overline{\mathrm{MS}} / \pi$ by solving the three-loop QCD renormalization group equation with the two-loop matching condition [72]:

$$
a^{(4)}\left(m_{b}\right)=a^{(5)}\left(m_{b}\right)+\frac{5}{36}\left[a^{(5)}\left(m_{b}\right)\right]^{3}
$$

at $\mu=m_{b}$. The relation between the $\overline{\mathrm{MS}}$ quark mass $\hat{m}_{q}\left(m_{q}\right)$ and the physical mass $m_{q}$ is given in ref. [73] as

$$
\hat{m}_{q}\left(m_{q}\right)=m_{q}\left[1+\frac{4}{3} a^{\left(n_{f}\right)}\left(m_{q}\right)+K_{q}\left[a^{\left(n_{f}\right)}\left(m_{q}\right)\right]^{2}\right]^{-1}
$$

with $K_{b} \approx 12.4\left(n_{f}=5\right)$ and $K_{c} \approx 13.3\left(n_{f}=4\right)$, for bottom and charm quarks. The following table summarizes the running quark masses, $\hat{m}_{b}(\mu)$ and $\hat{m}_{c}(\mu)$, for $\alpha_{s}\left(m_{Z}\right) \overline{\mathrm{MS}}=$ $0.11,0.12,0.13, m_{b}=4.7 \pm 0.2 \mathrm{GeV}, m_{c}=1.4 \pm 0.2 \mathrm{GeV}$ and $m_{Z}=91.187 \mathrm{GeV}$ (the 
difference $m_{b}-m_{c}$ is fixed to $3.3 \mathrm{GeV}$ [74 in evaluating $\left.\hat{m}_{c}(\mu)\right)$ :

\begin{tabular}{|l|ccc|}
\hline$\alpha_{s}\left(m_{Z}\right)_{\overline{\mathrm{MS}}}$ & 0.11 & 0.12 & 0.13 \\
\hline$m_{c}(\mathrm{GeV})$ & $1.40 \pm 0.20$ & $1.40 \pm 0.20$ & $1.40 \pm 0.20$ \\
\hline$\hat{m}_{c}\left(m_{c}\right)$ & $1.13 \pm 0.18$ & $1.03 \pm 0.19$ & $0.86 \pm 0.20$ \\
$\hat{m}_{c}\left(m_{b}\right)$ & $0.90 \pm 0.17$ & $0.76 \pm 0.17$ & $0.56 \pm 0.17$ \\
$\hat{m}_{c}\left(m_{Z}\right)$ & $0.65 \pm 0.13$ & $0.53 \pm 0.12$ & $0.37 \pm 0.12$ \\
\hline$m_{b}(\mathrm{GeV})$ & $4.70 \pm 0.20$ & $4.70 \pm 0.20$ & $4.70 \pm 0.20$ \\
\hline$\hat{m}_{b}\left(m_{b}\right)$ & $4.17 \pm 0.19$ & $4.06 \pm 0.18$ & $3.92 \pm 0.18$ \\
$\hat{m}_{b}\left(m_{Z}\right)$ & $3.05 \pm 0.16$ & $2.83 \pm 0.15$ & $2.59 \pm 0.15$ \\
\hline
\end{tabular}

The function $f\left(m_{t}\right)$ in the $O\left(\alpha_{s}^{2}\right)$ axial part of eq. (3.4b) is given by [56, 68] :

$$
f\left(m_{t}\right)=2 \ln \frac{m_{Z}}{m_{t}}-\frac{37}{12}+\frac{28}{81}\left(\frac{m_{Z}}{2 m_{t}}\right)^{2}-0.5767\left(\frac{m_{Z}}{2 m_{t}}\right)^{4}+0.7873\left(\frac{m_{Z}}{2 m_{t}}\right)^{6},
$$

The minus sign should be taken in front of $f\left(m_{t}\right)$ in eq. (3.4b) for $u, c$ quarks, and the plus sign for $d, s, b$ quarks. These formulae are sufficient to calculate the factors $C_{q V}$ and $C_{q A}$ as functions of $\alpha_{s}\left(m_{Z}\right), m_{b}$ and $m_{c}$. For charged leptons, the corresponding factors are

$$
\begin{aligned}
& C_{\ell V}=\frac{\beta_{\ell}\left(3-\beta_{\ell}^{2}\right)}{2}, \\
& C_{\ell A}=\beta_{\ell}^{3} .
\end{aligned}
$$

with

$$
\beta_{\ell}=\sqrt{1-\frac{4 m_{\ell}^{2}}{s}}
$$

The effect of the charged lepton masses is negligible except for the $\tau$ lepton.

Near the $Z$-pole, $s \sim m_{Z}^{2}$, the cross sections are sensitive to the total $Z$ width, $\Gamma_{Z}$, and hence it should be evaluated at two-loop level 441,44, 75. The $Z$ width is calculated in a similar way as the total cross section case (3.2):

$$
\begin{aligned}
& \Gamma_{Z}=\sum_{f} \Gamma_{f}, \\
& \Gamma_{f}=\frac{m_{Z}}{24 \pi}\left\{\left|M_{L}^{f}+M_{R}^{f}\right|^{2} \frac{C_{f V}}{2}+\left|M_{L}^{f}-M_{R}^{f}\right|^{2} \frac{C_{f A}}{2}\right\}\left(1+\frac{3}{4} Q_{f}^{2} \frac{\bar{\alpha}(s)}{\pi}\right),
\end{aligned}
$$

by using the $Z \rightarrow f_{\alpha} \overline{f_{\alpha}}$ decay amplitudes

$$
T\left(Z \rightarrow f_{\alpha} \overline{f_{\alpha}}\right)=M_{\alpha}^{f} \epsilon_{Z} \cdot J_{f_{\alpha}} .
$$

Here $\epsilon_{Z}^{\mu}$ is the normalized $Z$ wave function, $J_{f_{\alpha}}^{\mu}$ are the currents of eq. (2.1), and the scalar amplitudes $M_{\alpha}^{f}$ can be expressed by

$$
\begin{aligned}
M_{\alpha}^{f}= & \left(I_{3 f_{\alpha}}-Q_{f} \hat{s}^{2}\right)\left[\bar{g}_{Z}\left(m_{Z}^{2}\right)+\hat{g}_{Z} \operatorname{Re} \Gamma_{1}^{f_{\alpha}}\left(m_{Z}^{2}\right)\right] \\
& +\hat{g}_{Z} \operatorname{Re}\left[I_{3 f_{\alpha}}\left(\hat{c}^{2} \bar{\Gamma}_{2}^{f_{\alpha}}\left(m_{Z}^{2}\right)+\Gamma_{3}^{f_{\alpha}}\left(m_{Z}^{2}\right)\right)-Q_{f}\left(\bar{s}^{2}\left(m_{Z}^{2}\right)-\hat{s}^{2}\right)\right] .
\end{aligned}
$$


It is straightforward to evaluate the partial and total widths from the above formulae, once the three form factors $\bar{g}_{Z}^{2}\left(m_{Z}^{2}\right), \bar{s}^{2}\left(m_{Z}^{2}\right), \bar{\delta}_{b}\left(m_{Z}^{2}\right)$ and $\alpha_{s}\left(m_{Z}\right)$ are given. Fig. 3 shows the predicted $\Gamma_{Z}(\mathrm{GeV})$ in the plane of $\bar{s}^{2}\left(m_{Z}^{2}\right)$ and $\bar{g}_{Z}^{2}\left(m_{Z}^{2}\right)$ for $\alpha_{s}\left(m_{Z}\right)=0.11,0.12,0.13$ and $\bar{\delta}_{b}\left(m_{Z}^{2}\right)=0(\mathrm{a}),-0.01(\mathrm{~b})$ and $-0.02(\mathrm{c})$. In the SM, $\bar{\delta}_{b}\left(m_{Z}^{2}\right) \lesssim-0.003$ holds for all $m_{t}$ (see Fig. 目), $\bar{\delta}_{b}\left(m_{Z}^{2}\right)=-0.01(-0.02)$ for $m_{t} \approx 175(270) \mathrm{GeV}$. It It is clearly seen from the figure that $\Gamma_{Z}$ increases with growing $\alpha_{s}$ and $\bar{\delta}_{b}$, and that it remains roughly constant when $\alpha_{s}$ increases by 0.01 and, simultaneously, $\bar{\delta}_{b}$ decreases by about 0.006 . The net effect is a strong anti-correlation between the fitted $\alpha_{s}$ and $\bar{\delta}_{b}$ values (see section 4.1).

In the SM, all the form factors are calculable in terms of $m_{t}$ and $m_{H}$. In table 5 the SM predictions are shown for the partial and the total $Z$ widths for several $m_{t}$ and $m_{H}$ values, for $\alpha_{s}\left(m_{Z}\right)=0.12, \delta_{\alpha}=0, \bar{\delta}_{G}=0.0055$ and $\left(m_{b}, m_{c}\right)=(4.7,1.4) \mathrm{GeV}$. The numerical values turn out to be larger by about $1 / 5000$ than those quoted in ref. [44]. Uncertainties in our predictions are estimated as follows : (i) Change of $m_{b}$ and $m_{c}$ by $0.2 \mathrm{GeV}$ affects $\Gamma_{b}$ by less than $0.2 \mathrm{MeV}\left(\lesssim 1 / 2000\right.$ of $\left.\Gamma_{b}\right)$ and $\Gamma_{c}$ by about $0.03 \mathrm{MeV}$; (ii) Setting $\hat{g}_{Z}=\bar{g}_{Z}\left(m_{Z}^{2}\right)$ and $\hat{s}^{2}=\bar{s}^{2}\left(m_{Z}^{2}\right)$ in the amplitudes (3.18) affects the total width by about $0.2 \mathrm{MeV}$ for the $m_{t}$ and $m_{H}$ values of Table 5; (iii) If the imaginary parts in the amplitudes (3.18) are also included, the total width increases by about $0.01 \mathrm{MeV}$; (iv) QCD higher order effects may affect the hadronic widths at the level of $\alpha_{s}^{4} \sim 1 / 4000$; (v) The present uncertainty in $1 / \bar{\alpha}\left(m_{Z}^{2}\right), \delta_{\alpha}= \pm 0.1$ affects $\bar{s}^{2}\left(m_{Z}^{2}\right)$ by $\mp 0.00026$ (2.38b), and hence the $Z$ width by about $\pm 0.65 \mathrm{MeV}\left(\sim 1 / 3000\right.$ of $\left.\Gamma_{Z}\right)$. These uncertainties are still an order of magnitude smaller than the actual experimental error of $\Delta\left(\Gamma_{Z}\right)=7 \mathrm{MeV}$ [26] $\left(\Delta\left(\Gamma_{Z}\right) / \Gamma_{Z} \sim 0.003\right)$.

Note that we adopt the perturbative order $\alpha \alpha_{s}$ 46,54 56 corrections at $\alpha_{s}=\alpha_{s}\left(m_{Z}\right) \overline{\mathrm{MS}}$ in calculating all the SM predictions, since it allows the reader to reproduce our results straightforwardly. The effects of non-perturbative threshold corrections 60 62 may be

${ }^{4}$ The $m_{t}$-dependences of the electroweak $Z$ boson observables are not completely absorbed into the three form factors, $\bar{g}_{Z}^{2}\left(m_{Z}^{2}\right), \bar{s}^{2}\left(m_{Z}^{2}\right)$ and $\bar{\delta}_{b}\left(m_{Z}^{2}\right)$. Mild $m_{t}$-dependences remain in the two-loop QCD correction factor $f\left(m_{t}\right)$ of eq. (3.12) and in the $Z b_{L} b_{L}$ vertex function $\Gamma_{1}^{b_{L}}\left(m_{Z}^{2}\right)$ (Table 3$)$. When $\bar{\delta}_{b}\left(m_{Z}^{2}\right)$ is allowed to vary in the fit, these residual $m_{t}$-dependent terms are determined by using the SM $m_{t}$-dependence of the $\bar{\delta}_{b}\left(m_{Z}^{2}\right)$ form factor (see Fig. 11), which can be inverted approximately as $m_{t}(\mathrm{GeV})=$ $21.77 \sqrt{-10^{4} \bar{\delta}_{b}\left(m_{Z}^{2}\right)-9.9}-31.2$ valid in the region $75 \mathrm{GeV}<m_{t}<400 \mathrm{GeV}$. We set $m_{t}=75 \mathrm{GeV}$ for $\bar{\delta}_{b}\left(m_{Z}^{2}\right)>-0.0036$ and $m_{t}=400 \mathrm{GeV}$ for $\bar{\delta}_{b}\left(m_{Z}^{2}\right)<-0.0405$. With this prescription the parameter $\bar{\delta}_{b}\left(m_{Z}^{2}\right)$ covers the full $m_{t}$-dependences of the vertex corrections within the SM, while it still allows $\bar{\delta}_{b}\left(m_{Z}^{2}\right)$ to measure large new physics contributions to the $Z b_{L} b_{L}$ vertex because of the relatively mild $m_{t}$-dependences of the $f\left(m_{t}\right)$ and $\Gamma_{1}^{b_{L}}\left(m_{Z}^{2}\right)$ factors. 
accounted for by adjusting the effective top-quark mass to produce the same $T$ parameter value.

Once the $Z$ width, $\Gamma_{Z}$, is determined the formula (3.2) gives the total cross section for the process $e^{+} e^{-} \rightarrow f \bar{f}$ at all energies, up to the $\cos \theta$-dependence of the box form factors which can be safely neglected near the $Z$-pole. At LEP, the on-pole cross sections $\sigma_{f}^{0}$ are obtained after subtracting the $\gamma$-exchange contribution to the amplitudes. Because of this subtraction, we cannot simply compare $\sigma_{f}\left(m_{Z}^{2}\right)$ of eq. (3.2) with the corresponding published measurement. In fact, the subtraction procedure is not completely model-independent and the following two cases are examined : (i) In the amplitudes (2.2) only those terms multiplying the $Z$ propagator factor are retained; (ii) From the full amplitude (2.2) the $\gamma$-exchange amplitude $Q_{i} Q_{j}\left[\bar{e}^{2}\left(m_{Z}^{2}\right)-i \hat{e}^{2} \Delta_{\gamma \gamma}\left(m_{Z}^{2}\right)\right] / s$ is subtracted. The above two prescriptions differ by contributions from the $\gamma$ vertex corrections and the box corrections, but the numerical predictions for $\sigma_{h}^{0}$ are found to differ by at most $0.0003 \mathrm{nb}$ and are thus negligibly small compared to the actual experimental error of $\Delta\left(\sigma_{h}^{0}\right)=0.14 \mathrm{nb}$. The pole amplitudes (i), the term with the $Z$ propagator factor in eq. (2.2), are used below when confronting the theoretical predictions with the LEP/SLC experiments.

It must be pointed out here that the quantities quoted as $\sigma_{f}^{0}$ by the LEP electroweak working group [26] are not the peak cross sections as obtained above, but that they are rather defined by the following identities:

$$
\sigma_{f}^{0}(\mathrm{LEP}) \equiv \frac{12 \pi}{m_{Z}^{2}} \frac{\Gamma_{e} \Gamma_{f}}{\Gamma_{Z}^{2}} .
$$

This quantity does not agree with the pole cross section $\sigma_{f}^{0}$ as calculated above, but agrees rather accurately with the modified expression:

$$
\sigma_{f}^{0}(\mathrm{LEP}) \approx \sigma_{f}^{0} \cdot\left(1+\frac{3}{4} \frac{\bar{\alpha}(s)}{\pi}\right)
$$

For example, the SM predictions for $m_{t}=175 \mathrm{GeV}, m_{H}=100 \mathrm{GeV}, \alpha_{s}\left(m_{Z}\right)=0.12$ and

${ }^{5}$ We thank T. Mori for pointing out our misunderstanding of $\sigma_{h}^{0}$ affecting the earlier version of the present work. The notation of the LEP electroweak working group is misleading, since ref. [26] does not explicitly state that their $\sigma_{h}^{0}$ value is not the peak cross section. In order to avoid any ambiguity it would be better to call this quantity $\left(12 \pi / m_{Z}^{2}\right) \Gamma_{e} \Gamma_{h} / \Gamma_{Z}^{2}$ and explain precisely from which experimental quantities it is calculated. It is also desirable to publish the total hadronic cross sections at $\sqrt{s}=m_{Z}$ without subtracting the $\gamma$-exchange contributions, since the full total cross sections can be calculated unambiguously. 
$\delta_{\alpha}=0$ give :

\begin{tabular}{|c|c|c|r|}
\hline$f$ & $\sigma_{f}^{0}$ & $\sigma_{f}^{0} \cdot\left(1+\frac{3}{4} \frac{\bar{\alpha}\left(m_{Z}^{2}\right)}{\pi}\right)$ & $\sigma_{f}^{0}(\mathrm{LEP})$ \\
\hline$\ell=e, \mu$ & $1.995 \mathrm{nb}$ & $1.998 \mathrm{nb}$ & $1.997 \mathrm{nb}$ \\
\hline$h$ & $41.399 \mathrm{nb}$ & $41.476 \mathrm{nb}$ & $41.463 \mathrm{nb}$ \\
\hline$b$ & $8.928 \mathrm{nb}$ & $8.945 \mathrm{nb}$ & $8.942 \mathrm{nb}$ \\
\hline
\end{tabular}

The right-hand side of (3.20) reproduces the LEP definition (3.19) with an accuracy of $1 / 3000$, while the peak cross sections $\sigma_{f}^{0}$ as obtained from eq. (3.2) with the $Z$-pole part of the amplitudes are off by about $1 / 1000$ to $1 / 600$. The former uncertainty of about $1 / 3000$ is typically on the order of the higher order corrections, while the latter difference, especially the difference between $\sigma_{h}^{0}$ and $\sigma_{h}^{0}(\mathrm{LEP})$, shows up clearly in the fit as a significant shift in the fitted $\bar{\delta}_{b}\left(m_{Z}^{2}\right)$ and $\alpha_{s}$ values.

Figs. 1, 5, 6 show

$$
\begin{aligned}
& \sigma_{h}^{0}(\mathrm{LEP})=\sum_{f=u, d, s, c, b} \sigma_{f}^{0}(\mathrm{LEP}), \\
& R_{\ell}=\sigma_{h}^{0}(\mathrm{LEP}) / \sigma_{\ell}^{0}(\mathrm{LEP})=\Gamma_{h} / \Gamma_{\ell}, \\
& R_{b}=\sigma_{b}^{0}(\mathrm{LEP}) / \sigma_{h}^{0}(\mathrm{LEP})=\Gamma_{b} / \Gamma_{h},
\end{aligned}
$$

respectively, in the plane of $\bar{s}^{2}\left(m_{Z}^{2}\right)$ and $\bar{\delta}_{b}\left(m_{Z}^{2}\right)$ for $\alpha_{s}\left(m_{Z}\right)=0.11,0.12$ and 0.13 . All the three quantities turn out to be almost completely independent of $\bar{g}_{Z}^{2}\left(m_{Z}^{2}\right)$, as the predictions at $\bar{g}_{Z}^{2}\left(m_{Z}^{2}\right)=0.55$ (solid lines) and $\bar{g}_{Z}^{2}\left(m_{Z}^{2}\right)=0.57$ (dashed lines) are almost degenerate. Fig. 4 shows that $\sigma_{h}^{0}$ is sensitive to both $\alpha_{s}$ and $\bar{\delta}_{b}$, but an increase of $\alpha_{s}\left(m_{Z}\right)$ by 0.01 can be compensated by a simultaneous decrease of $\bar{\delta}_{b}$ by about 0.006 , just as for $\Gamma_{Z}$. Fig. 5 shows that the ratio $R_{\ell}$ is only sensitive to a linear combination of $\bar{s}^{2}\left(m_{Z}^{2}\right)$ and $\bar{\delta}_{b}\left(m_{Z}^{2}\right)$. At fixed $\bar{s}^{2}\left(m_{Z}^{2}\right)$, the correlated change of $\alpha_{s}$ and $\bar{\delta}_{b}$ leaving $\Gamma_{Z}$ and $\sigma_{h}^{0}$ unchanged, keeps also the $R_{\ell}$ value roughly unchanged. The reason for this behaviour is in the fact that the $\alpha_{s}$-dependences of the three $Z$-resonance observables, $\Gamma_{Z}, \sigma_{h}^{0}$ and $R_{l}$, are solely contained in just the quantity $\Gamma_{h}$ which depends on $\alpha_{s}$ and $\delta_{b}$ approximately in the combination

$$
\bar{\delta}_{b}\left(m_{Z}^{2}\right)+0.6 \alpha_{s}\left(m_{Z}\right) .
$$

Hence, in order to get $\alpha_{s}$ independently of $\bar{\delta}_{b}$, the measurement sensitive to another combination is required. For instance, Fig. 6 shows that the ratio $R_{b}$ does measure $\bar{\delta}_{b}\left(m_{Z}^{2}\right)$ rather independently of $\alpha_{s}$ and $\bar{s}^{2}\left(m_{Z}^{2}\right)$. An accurate measurement of $R_{b}$ offers the key to disentangle $\alpha_{s}$ and $\bar{\delta}_{b}$ (see also section 4.1 ).

The asymmetries on the $Z$-pole provide the measurement of the universal parameter $\bar{s}^{2}\left(m_{Z}^{2}\right)$ almost independently of $\bar{g}_{Z}^{2}\left(m_{Z}^{2}\right)$ and $\bar{\delta}_{b}\left(m_{Z}^{2}\right)$ and with little or no dependence on the QCD coupling $\alpha_{s}$. 
The forward-backward (FB) asymmetry is given by

$$
A_{\mathrm{FB}}^{0, \ell}=\frac{3}{4} \frac{\left|M_{L L}^{e \ell}\right|^{2}+\left|M_{R R}^{e \ell}\right|^{2}-\left|M_{L R}^{e \ell}\right|^{2}-\left|M_{R L}^{e \ell}\right|^{2}}{\left|M_{L L}^{e \ell}\right|^{2}+\left|M_{R R}^{e \ell}\right|^{2}+\left|M_{L R}^{e \ell}\right|^{2}+\left|M_{R L}^{e \ell}\right|^{2}},
$$

for leptons, and

$$
A_{\mathrm{FB}}^{0, q}=\frac{3}{4} \frac{2 \beta_{q}\left\{\left|M_{L L}^{e q}\right|^{2}+\left|M_{R R}^{e q}\right|^{2}-\left|M_{L R}^{e q}\right|^{2}-\left|M_{R L}^{e q}\right|^{2}\right\}}{\frac{3-\beta_{q}^{2}}{2}\left\{\left|M_{L L}^{e q}+M_{L R}^{e q}\right|^{2}+\left|M_{R L}^{e q}+M_{R R}^{e q}\right|^{2}\right\}+\beta_{q}^{2}\left\{\left|M_{L L}^{e q}-M_{L R}^{e q}\right|^{2}+\left|M_{R L}^{e q}-M_{R R}^{e q}\right|^{2}\right\}},
$$

for quarks $(q=b, c)$. Here, the physical heavy quark masses $m_{q}$ are used in the factor $\beta_{q}=\sqrt{1-4 m_{q}^{2} / m_{Z}^{2}}$. The QCD corrections for the FB asymmetries [76] have not been included in eq. (3.27). The reported asymmetries from LEP $A_{\mathrm{FB}}^{0, b}(\mathrm{LEP})$ and $A_{\mathrm{FB}}^{0, c}(\mathrm{LEP})$ have been corrected for these effects assuming a linear $\alpha_{s}$-dependence and $\alpha_{s}=0.12$. Therefore, we estimate the LEP asymmetries for a given value of $\alpha_{s}\left(m_{Z}\right)$ by using the following simple formula :

$$
A_{\mathrm{FB}}^{0, q}(\mathrm{LEP})=A_{\mathrm{FB}}^{0, q} \frac{1+k_{A}\left(\frac{\alpha_{s}}{\pi}\right)}{1+k_{A} \frac{0.12}{\pi}}
$$

with $k_{A}=0.75$ [26]. The uncertainty in the coefficient $\Delta k_{A}= \pm 0.25$ affects the above $\alpha_{s}$ dependence by less than $1 / 1000$ in the range $0.11<\alpha_{s}\left(m_{Z}\right)<0.13$. The QCD correction depends on details of the final charm and bottom quark tagging procedure, and hence it is desirable to have the $\alpha_{s}$-dependence of the corrected asymmetry value from each experiment.

The $\tau$ polarization asymmetry is defined by the ratio of the left- and right-handed $\tau$ pair cross sections :

$$
P_{\tau}=\frac{\sigma_{\tau_{R}}-\sigma_{\tau_{L}}}{\sigma_{\tau_{R}}+\sigma_{\tau_{L}}}
$$

By neglecting the $\tau$ mass one finds

$$
P_{\tau}=\frac{\left|M_{L R}^{e \ell}\right|^{2}+\left|M_{R R}^{e \ell}\right|^{2}-\left|M_{L L}^{e \ell}\right|^{2}-\left|M_{R L}^{e \ell}\right|^{2}}{\left|M_{L R}^{e \ell}\right|^{2}+\left|M_{R R}^{e \ell}\right|^{2}+\left|M_{L L}^{e \ell}\right|^{2}+\left|M_{R L}^{e \ell}\right|^{2}} .
$$

Likewise, the left-right beam polarization asymmetry is defined by

$$
A_{\mathrm{LR}}=\frac{\sum_{f}\left(\sigma_{f}^{L}-\sigma_{f}^{R}\right)}{\sum_{f}\left(\sigma_{f}^{L}+\sigma_{f}^{R}\right)} .
$$


where the cross sections for completely polarized beam are expressed in terms of the helicity amplitudes by

$$
\begin{aligned}
\sigma_{f}^{L} & \equiv \sigma\left(e_{L}^{-} e_{R}^{+} \rightarrow f \bar{f}\right) \\
& =\frac{s}{24 \pi}\left\{\left|M_{L L}^{e f}+M_{L R}^{e f}\right|^{2} \frac{C_{f V}}{2}+\left|M_{L L}^{e f}-M_{L R}^{e f}\right|^{2} \frac{C_{f A}}{2}\right\}\left(1+\frac{3}{4} Q_{f}^{2} \frac{\bar{\alpha}(s)}{\pi}\right), \\
\sigma_{f}^{R} & \equiv \sigma\left(e_{R}^{-} e_{L}^{+} \rightarrow f \bar{f}\right) \\
& =\frac{s}{24 \pi}\left\{\left|M_{R L}^{e f}+M_{R R}^{e f}\right|^{2} \frac{C_{f V}}{2}+\left|M_{R L}^{e f}-M_{R R}^{e f}\right|^{2} \frac{C_{f A}}{2}\right\}\left(1+\frac{3}{4} Q_{f}^{2} \frac{\bar{\alpha}(s)}{\pi}\right) .
\end{aligned}
$$

The cross section for the electron beam polarization $P_{e}$ is then

$$
\sigma_{f}\left(P_{e}\right)=\frac{1-P_{e}}{2} \sigma_{f}^{L}+\frac{1+P_{e}}{2} \sigma_{f}^{R}
$$

We comment here that the factorization identities

$$
\begin{aligned}
& A_{\mathrm{LR}}=-P_{\tau}, \\
& A_{\mathrm{FB}}^{0, \ell}=\frac{3}{4}\left(P_{\tau}\right)^{2},
\end{aligned}
$$

do not hold exactly even in our $Z$-pole approximation to the amplitudes (2.2), since they do not factorize into $Z$ production and $Z$ decay amplitudes at $s=m_{Z}^{2}$. We find for instance for the SM predictions at $m_{t}=175 \mathrm{GeV}, m_{H}=100 \mathrm{GeV}, \alpha_{s}\left(m_{Z}\right)=0.12$ and $\delta_{\alpha}=0$ :

\begin{tabular}{|c|c|}
\hline $\bar{s}^{2}\left(m_{Z}^{2}\right)$ & 0.23040 \\
\hline$A_{\mathrm{LR}}$ & 0.14801 \\
$-P_{\tau}$ & 0.14802 \\
\hline$A_{\mathrm{FB}}^{0, \ell}$ & 0.01667 \\
$\frac{3}{4}\left(P_{\tau}\right)^{2}$ & 0.01643 \\
\hline
\end{tabular}

The identity (3.34) holds rather accurately, but the identity (3.35) is violated by a factor of $1.4 \%$. This is mainly because of the subtle cancellation among the squared amplitudes of eq. (3.26) rendering the asymmetry $A_{\mathrm{FB}}^{\ell}$ sensitive to our detailed treatment of the order $\alpha^{2}$ effects such as the treatment of the imaginary part and the choice of the couplings $\hat{g}_{Z}^{2}$ and $\hat{s}^{2}$.

In Fig. 17, all asymmetry parameters on the $Z$-pole are plotted as functions of $\bar{s}^{2}\left(m_{Z}^{2}\right)$. For each asymmetry, the contributions from both the $\gamma$-pole and $Z$-pole terms are examined using the following helicity amplitudes : (i) The full helicity amplitudes (2.2) including the $\gamma$ and $Z$ exchange as well as the box contributions. (ii) The helicity amplitudes obtained from the full amplitudes (2.2) by subtracting the real and imaginary parts of the $\gamma$ exchange contribution $Q_{i} Q_{j}\left[\bar{e}^{2}\left(m_{Z}^{2}\right)-i \hat{e}^{2} \Delta_{\gamma \gamma}\left(m_{Z}^{2}\right)\right] / s$. (iii) The helicity 
amplitude retaining only the $Z$-pole term, the term multiplying the $Z$ propagator factor in eq. (2.2). (iv) The helicity amplitude in the improved Born approximation (IBA) of the $Z$-exchange amplitudes :

$$
\left(M_{\alpha \beta}^{e f}\right)_{\mathrm{IBA}}=\frac{\bar{g}_{Z}^{2}\left(m_{Z}^{2}\right)\left[I_{3 e_{\alpha}}-Q_{e} \bar{s}^{2}\left(m_{Z}^{2}\right)\right]\left[I_{3 f_{\beta}}-Q_{f} \bar{s}^{2}\left(m_{Z}^{2}\right)\right]}{s-m_{Z}^{2}+i s \frac{\Gamma_{Z}}{m_{Z}}},
$$

on the $Z$-pole $s=m_{Z}^{2}$. In Fig. 7, the predictions of (i) are denoted by 'Full', (ii) by 'Full $-\gamma$ ', (iii) by ' $Z$ only', and (iv) by 'IBA'. The prescriptions (ii) and (iii) give almost identical predictions, and we adopt (iii) in the fit. It is worth noting that the subtraction of the $\gamma$-exchange amplitudes affects the asymmetry $A_{\mathrm{FB}}^{\ell}$ significantly, but not the other asymmetries. Note particularly that the IBA gives consistently larger asymmetries by as much as $10 \%$ for $A_{\mathrm{FB}}^{\ell}$, and by about $5 \%$ for the rest. Hence, the 'process-dependent' effective $\sin ^{2} \theta_{W}$ factor determined from each asymmetry by making use of the IBA-like formula (3.37) differs significantly from the process-independent universal form factor $\bar{s}^{2}\left(m_{Z}^{2}\right)$. We find approximately,

$$
\begin{aligned}
& \sin ^{2} \theta_{W}^{\mathrm{eff}}\left(A_{\mathrm{FB}}^{0, \ell}\right) \approx \bar{s}^{2}\left(m_{Z}^{2}\right)+0.0009, \\
& \sin ^{2} \theta_{W}^{\mathrm{eff}}\left(A_{\mathrm{LR}}^{0}\right) \approx \bar{s}^{2}\left(m_{Z}^{2}\right)+0.0010, \\
& \sin ^{2} \theta_{W}^{\mathrm{e} f}\left(A_{\mathrm{FB}}^{0, b}\right) \approx \bar{s}^{2}\left(m_{Z}^{2}\right)+0.0010, \\
& \sin ^{2} \theta_{W}^{\mathrm{eff}}\left(A_{\mathrm{FB}}^{0, c}\right) \approx \bar{s}^{2}\left(m_{Z}^{2}\right)+0.0009 .
\end{aligned}
$$

A related study is found in ref. [77].

In the SM, all the form factors $\bar{g}_{Z}^{2}\left(m_{Z}^{2}\right), \bar{s}^{2}\left(m_{Z}^{2}\right)$ and $\bar{\delta}_{b}\left(m_{Z}^{2}\right)$ are calculable as functions of $m_{t}$ and $m_{H}$ (see appendix $\mathrm{C}$ for details). The main uncertainty in these calculations appears in the parameter $\delta_{\alpha}$ (2.31) which parametrizes the uncertainty in the hadronic vacuum polarization contribution to $1 / \bar{\alpha}\left(m_{Z}^{2}\right)$. Hence, all $Z$ parameters can be predicted accurately in the SM as functions of four parameters: $m_{t}, m_{H}, \alpha_{s}$ and $\delta_{\alpha}$.

Figs. 8 shows the $m_{t}$-dependence of all $Z$ parameters for three $m_{H}$ values $60 \mathrm{GeV}$ (dashed lines), $300 \mathrm{GeV}$ (solid lines) and $1000 \mathrm{GeV}$ (dash-dotted lines), at $\alpha_{s}\left(m_{Z}\right)=0.11$, $0.12,0.13$ and $\delta_{\alpha}=0\left(1 / \bar{\alpha}\left(m_{Z}^{2}\right)=128.72\right)$. Shown by horizontal lines are the experimental data from LEP [26] and SLC [31] (see sections 4 and 6). The $m_{t}$-dependence is sizeable for all the observables. In $R_{b}$ and $\sigma_{h}^{0}$, the $m_{t}$-dependence comes mainly from the $Z b_{L} b_{L}$ form factor $\bar{\delta}_{b}\left(m_{Z}^{2}\right)$, and hence these parameters have little sensitivity to $m_{H}$ (see Fig. 国). The $m_{t}$-dependences of all asymmetry parameters including $P_{\tau}$ come from the form factor $\bar{s}^{2}\left(m_{Z}^{2}\right) . \quad R_{\ell}$ receives $m_{t}$-dependences from both $\bar{\delta}_{b}\left(m_{Z}^{2}\right)$ and $\bar{s}^{2}\left(m_{Z}^{2}\right)$. Finally, the total $Z$ width is the only quantity sensitive to the form factor $\bar{g}_{Z}^{2}\left(m_{Z}^{2}\right)$. In conclusion, the 
$m_{t}$-dependence of $\Gamma_{Z}$ is a combined effect of all three form factors $\bar{g}_{Z}^{2}\left(m_{Z}^{2}\right), \bar{s}^{2}\left(m_{Z}^{2}\right)$ and $\bar{\delta}_{b}\left(m_{Z}^{2}\right)$.

Likewise, Fig. 9 shows the $\alpha_{s}$ dependences of the hadronic $Z$ parameters for the three $m_{t}$ values $100 \mathrm{GeV}$ (dashed lines), $150 \mathrm{GeV}$ (solid lines) and $200 \mathrm{GeV}$ (dash-dotted lines), all at $m_{H}=100 \mathrm{GeV}$ and $\delta_{\alpha}=0$. It can be seen that the ratio $R_{b}$ and the asymmetries $A_{\mathrm{FB}}^{0, b}$ and $A_{\mathrm{FB}}^{0, c}$ are almost independent of $\alpha_{s} . \Gamma_{Z}$ and $R_{\ell}$ grow linearly with $\alpha_{s}$ because of the final state QCD correction factor (3.4). $\sigma_{h}^{0}$ decreases with increasing $\alpha_{s}$, since it is proportional to the factor $\Gamma_{h} / \Gamma_{Z}^{2}$. The ratio $R_{\ell}$ exhibits the strongest dependence to $\alpha_{s}$. As emphasized above, however, the $\alpha_{s}$-dependences of all $Z$ observables are approximately proportional to a common factor $\bar{\delta}_{b}\left(m_{Z}^{2}\right)+0.6 \alpha_{s}\left(m_{Z}\right)$, and hence either an accurate determination of $\bar{\delta}_{b}\left(m_{Z}^{2}\right)$ (via $R_{b}$ ) or else the assumption of SM dominance to the form factor $\bar{\delta}_{b}\left(m_{Z}^{2}\right)$ is crucial for the extraction of $\alpha_{s}\left(m_{Z}\right)$ from these experiments.

\subsection{Low energy neutral current experiments}

The data of four types of low energy neutral current experiments are analysed : neutrino-nucleon scattering $\left(\nu_{\mu}-q\right)$, neutrino-electron scattering $\left(\nu_{\mu}-e\right)$, atomic parity violation (APV), and polarized electron-deuteron scattering $(e-\mathrm{D})$. Theoretical predictions are given for all model-independent parameters [24, 88, 79] characterizing the electroweak low energy neutral current experiments. They are the effective $\nu_{\mu}-q$ coupling factors [78]

$$
g_{L}^{2}, \quad g_{R}^{2}, \quad \delta_{L}^{2}, \quad \delta_{R}^{2},
$$

for the $\nu_{\mu}-q$ scattering experiments, the effective neutral current parameters [79]

$$
\rho_{\nu e}, s_{\nu e}^{2}
$$

for the $\nu_{\mu}-e$ scattering experiments, the weak charge of nuclei [B]]

$$
Q_{W}(A, Z),
$$

for parity violation in atoms, and the effective neutral current couplings [24]

$$
2 C_{1 u}-C_{1 d}, \quad 2 C_{2 u}-C_{2 d},
$$

for the $e-\mathrm{D}$ polarization asymmetry. Definitions of these model-independent parameters are given below and re-expressed in terms of the helicity amplitudes of eq. (2.2).

In this subsection terms of order $\alpha \cdot\left(q^{2} / m_{W}^{2}\right)$ are neglected, while keeping terms of order $q^{2} / m_{W}^{2}$ and $\alpha \cdot\left(m_{f}^{2} / q^{2}\right)$. The generic amplitude for the process $i j \rightarrow i j$ follows then 
from eq. (2.2) :

$$
\begin{aligned}
M_{i j}^{N C}=\frac{1}{q^{2}}\left\{\left(Q_{i} Q_{j}\right)\right. & {\left[\bar{e}^{2}\left(q^{2}\right)+\hat{e}^{2} \Gamma_{1}^{i}\left(q^{2}\right)+\hat{e}^{2} \Gamma_{1}^{j}\left(q^{2}\right)\right] } \\
& \left.+\left(Q_{i} I_{3 j}\right) \hat{e}^{2} \bar{\Gamma}_{2}^{j}\left(q^{2}\right)+\left(Q_{j} I_{3 i}\right) \hat{e}^{2} \bar{\Gamma}_{2}^{i}\left(q^{2}\right)\right\} \\
+\frac{1}{q^{2}-m_{Z}^{2}}\{ & \left(I_{3 i}-Q_{i} \hat{s}^{2}\right)\left(I_{3 j}-Q_{j} \hat{s}^{2}\right) \bar{g}_{Z}^{2}(0) \\
& -\left(I_{3 i}-Q_{i} \hat{s}^{2}\right) Q_{j} \hat{g}_{Z}^{2}\left[\bar{s}^{2}\left(q^{2}\right)-\hat{s}^{2}\right] \\
& \left.-\left(I_{3 j}-Q_{j} \hat{s}^{2}\right) Q_{i} \hat{g}_{Z}^{2}\left[\bar{s}^{2}\left(q^{2}\right)-\hat{s}^{2}\right]\right\} \\
+B_{i j}^{N C}(0,0) & +O\left(\hat{e}^{2} \frac{q^{2}}{m_{W}^{2}}\right) .
\end{aligned}
$$

All electroweak observables of the low energy neutral current sector are calculated by using the above approximation. Contributions from the neglected terms are completely negligible. The numerical predictions for all observables (3.39)-(3.42) depend on just the two universal charge form factors $\bar{s}^{2}(0)$ and $\bar{g}_{Z}^{2}(0)$, since the running of the charge form factors $1 / \bar{\alpha}\left(q^{2}\right)-1 / \alpha$ and $\bar{s}^{2}\left(q^{2}\right) / \bar{\alpha}\left(q^{2}\right)-\bar{s}^{2}(0) / \alpha$ at low energies $\left|q^{2}\right| \ll m_{Z}^{2}$ are governed completely by known physics only and are hence accurately calculable (see appendix B). Although the expression (3.43) with the $\overline{\mathrm{MS}}$ coupling normalization (2.19) is used in all numerical calculations presented below, we often quote below a slightly more compact expression that is obtained from eq. (3.43) by dropping the terms proportional to $\left[\bar{s}^{2}\left(q^{2}\right)-\hat{s}^{2}\right]$ and replacing $\hat{s}^{2}$ by $\bar{s}^{2}\left(q^{2}\right)$ in the term multiplying the $Z$ propagator factor. This is a valid approximation to eq. (3.43) differing only by terms of order $\hat{g}_{Z}^{2}\left[\bar{s}^{2}\left(q^{2}\right)-\hat{s}^{2}\right]^{2}$.

\subsubsection{Neutral currents in $\nu_{\mu}-q$ scattering}

The neutral current data from the $\nu-q$ scattering experiments can be conveniently parametrized by the four model-independent parameters [78]

$$
\begin{aligned}
& g_{\alpha}^{2} \equiv u_{\alpha}^{2}+d_{\alpha}^{2}, \\
& \delta_{\alpha}^{2} \equiv u_{\alpha}^{2}-d_{\alpha}^{2},
\end{aligned}
$$

for $\alpha=L$ or $R$. The effective chiral couplings $q_{\alpha}\left(=u_{L}, d_{L}, u_{R}, d_{R}\right)$ can be directly expressed in terms of the helicity amplitudes of eq. (3.43) by

$$
q_{\alpha}=-\frac{M_{L \alpha}^{\nu_{\mu} q}}{2 \sqrt{2} G_{\text {c.c. }}} \quad(q=u, d ; \alpha=L, R),
$$

with the notation $M_{\alpha \beta}^{i j} \equiv M_{i_{\alpha} j_{\beta}}^{N C}$. The amplitudes (3.43) can then be written in compact form :

$$
M_{L \alpha}^{\nu_{\mu} q}=\frac{1}{2} Q_{q} \hat{e}^{2} \frac{\bar{\Gamma}_{2}^{\nu}(t)}{t}+\frac{1}{2}\left[I_{3 q_{\alpha}}-Q_{q} \bar{s}^{2}(t)\right] \frac{\bar{g}_{Z}^{2}(0)}{t-m_{Z}^{2}}+B_{L \alpha}^{\nu_{\mu} q}(0,0)
$$


and the charged current factor is approximated by

$$
G_{\text {c.c. }}=G_{F} \frac{\left[1+\delta_{\text {c.c. }}\right]^{1 / 2}}{1+\frac{\langle-t\rangle_{\text {c.c. }}}{m_{W}^{2}}} .
$$

The QED correction factor $\delta_{\text {c.c. }}$ is accounted for (following Sirlin and Marciano 81]) by,

$$
\begin{aligned}
\delta_{\text {c.c. }} & =\frac{\alpha}{\pi}\left[\ln \frac{m_{Z}^{2}}{2\langle-t\rangle_{\text {c.c. }}}+2\right] \\
& \approx 0.017 \quad \text { for }\langle-t\rangle_{\text {c.c. }}=20 \mathrm{GeV}^{2} .
\end{aligned}
$$

Note that the leading logarithm approach of ref. [36] gives

$$
\begin{aligned}
\delta_{\text {c.c. }} & =\frac{\alpha}{\pi} \ln \frac{m_{W}^{2}}{\langle-t\rangle_{\text {c.c. }}} \\
& \approx 0.013 \quad \text { for }\langle-t\rangle_{\text {c.c. }}=20 \mathrm{GeV}^{2},
\end{aligned}
$$

for the above correction factor. In our numerical calculation we adopt the factor (3.48b). The $\nu_{\mu}$ charge radius factor $\bar{\Gamma}_{2}^{\nu}(t) / t$ and the box form factors $B_{L \alpha}^{\nu_{\mu} q}(0,0)$ in the amplitude (3.46) can easily be read off from the generic expressions in appendix A :

$$
\frac{\bar{\Gamma}_{2}^{\nu}(t)}{t}=\frac{1}{m_{W}^{2}} \frac{\hat{g}^{2}}{16 \pi^{2}} \bar{J}_{W}\left(t ; m_{\mu}\right)
$$

with

$$
\begin{aligned}
\bar{J}_{W}\left(t ; m_{\mu}\right) & =4 F_{3}\left(t ; m_{\mu}, m_{\mu}\right)-\frac{2}{3} \ln m_{W}^{2}-1 \\
& =\frac{2}{3} \ln \frac{-t}{m_{W}^{2}}-\frac{19}{9}+O\left(\frac{t}{m_{W}^{2}}, \frac{m_{\mu}^{2}}{t}\right),
\end{aligned}
$$

from eq. (A.27b) and

$$
\begin{array}{ll}
B_{L L}^{\nu_{\mu} u}(0,0)= & -\frac{\hat{g}^{4}}{64 \pi^{2} m_{W}^{2}}+\frac{3 \hat{g}_{Z}^{4}}{64 \pi^{2} m_{Z}^{2}}\left(\frac{1}{2}-\frac{2}{3} \hat{s}^{2}\right)^{2}, \\
B_{L R}^{\nu_{\mu} u}(0,0)= & -\frac{3 \hat{g}_{Z}^{4}}{64 \pi^{2} m_{Z}^{2}}\left(-\frac{2}{3} \hat{s}^{2}\right)^{2}, \\
B_{L L}^{\nu_{\mu} d}(0,0)= & \frac{\hat{g}^{4}}{16 \pi^{2} m_{W}^{2}}+\frac{3 \hat{g}_{Z}^{4}}{64 \pi^{2} m_{Z}^{2}}\left(-\frac{1}{2}+\frac{1}{3} \hat{s}^{2}\right)^{2}, \\
B_{L R}^{\nu_{\mu} d}(0,0)= & -\frac{3 \hat{g}_{Z}^{4}}{64 \pi^{2} m_{Z}^{2}}\left(\frac{1}{3} \hat{s}^{2}\right)^{2},
\end{array}
$$

from eq. (A.35). These expressions are sufficient to evaluate the helicity amplitudes (3.46) as functions of $\bar{s}^{2}(0)$ and $\bar{g}_{Z}^{2}(0)$, for the $\overline{\mathrm{MS}}$ coupling normalization of eq. (2.19). We set $m_{W}=80.24 \mathrm{GeV}$ and $m_{Z}=91.187 \mathrm{GeV}$ in all numerical calculations. 


$$
\begin{aligned}
& \text { At }\langle-t\rangle_{\text {n.c. }}=20 \mathrm{GeV}^{2}, \\
& \qquad \frac{\hat{e}^{2}}{16 \pi^{2}} \bar{J}_{W}\left(t=-20 \mathrm{GeV}^{2} ; m_{\mu}\right) \approx-0.0037,
\end{aligned}
$$

and the $q_{\alpha}$ 's are approximated as

$$
q_{\alpha} \approx 0.9923 \bar{\rho}\left[I_{3 q_{\alpha}}-Q_{q} \bar{s}^{2}(t)\right]+ \begin{cases}+0.0031 & \left(q_{\alpha}=u_{L}\right) \\ +0.0026 & \left(q_{\alpha}=u_{R}\right) \\ -0.0074 & \left(q_{\alpha}=d_{L}\right) \\ -0.0012 & \left(q_{\alpha}=d_{R}\right)\end{cases}
$$

for the $\overline{\mathrm{MS}}$ coupling normalization of eq. (2.19). Here the universal $\bar{\rho}$ parameter is defined by

$$
\bar{\rho} \equiv \frac{\bar{g}_{Z}^{2}(0)}{4 \sqrt{2} G_{F} m_{Z}^{2}}=\frac{1}{1+\bar{\delta}_{G}-\alpha T} \approx \frac{\bar{g}_{Z}^{2}(0)}{0.54864} .
$$

The relation between the form factor $\bar{g}_{Z}^{2}(0)$ and the $T$ parameter is seen in eq. (2.36a). The running of $\bar{s}^{2}(t)$ is estimated as

$$
\begin{aligned}
\bar{s}^{2}\left(t=-20 \mathrm{GeV}^{2}\right) & \approx \frac{\bar{\alpha}\left(t=-20 \mathrm{GeV}^{2}\right)}{\alpha}\left[\bar{s}^{2}(0)-0.0097\right] \\
& \approx 1.0295 \bar{s}^{2}(0)-0.0100 .
\end{aligned}
$$

The approximations (3.54)-(3.56) are found to give excellent numerical predictions for all $q_{\alpha}$ as functions of the two charge form factors, $\bar{s}^{2}(0)$ and $\bar{g}_{Z}^{2}(0)$.

The major effects of radiative corrections can be made transparent by parametrizing the model-independent coupling factors of eq. (3.45) in terms of the effective couplings $\rho_{\nu q}$ and $s_{\nu q}^{2}$ of ref. [82]:

$$
\begin{aligned}
& u_{L}=\rho_{\nu q}\left(\frac{1}{2}-\frac{2}{3} s_{\nu q}^{2}\right)+\Delta_{u_{L}}, \\
& d_{L}=\rho_{\nu q}\left(-\frac{1}{2}+\frac{1}{3} s_{\nu q}^{2}\right)+\Delta_{d_{L}}, \\
& u_{R}=\rho_{\nu q}\left(-\frac{2}{3} s_{\nu q}^{2}\right)+\Delta_{u_{R}}, \\
& d_{R}=\rho_{\nu q}\left(\frac{1}{3} s_{\nu q}^{2}\right)+\Delta_{d_{R}} .
\end{aligned}
$$

The extra terms $\Delta_{q_{\alpha}}$ are fixed such that they do not interfere with the leading terms in the most accurately measured quantities, that is, $g_{L}^{2}$ and $g_{R}^{2}$. One finds

$$
\begin{aligned}
& \Delta_{u_{L}}=\frac{\hat{g}_{Z}^{2}}{8 \pi^{2}}\left(\hat{c}^{2}+\frac{\hat{s}^{2}}{3}\right) a_{\beta L}, \\
& \Delta_{d_{L}}=\frac{\hat{g}_{Z}^{2}}{8 \pi^{2}}\left(\hat{c}^{2}-\frac{\hat{s}^{2}}{3}\right) a_{\beta L}, \\
& \Delta_{d_{R}}=2 \Delta_{u_{R}}=\frac{\hat{g}_{Z}^{2}}{8 \pi^{2}} \frac{\hat{s}^{4}}{5},
\end{aligned}
$$


with

$$
a_{\beta L}=-\frac{1}{2 \hat{c}^{2}}\left(\frac{9}{8}-\frac{3}{2} \hat{s}^{2}+\frac{8}{15} \hat{s}^{4}\right) .
$$

The radiatively corrected amplitudes can then be expressed approximately in terms of the effective strengths ' $\rho_{\nu q}$ ' of the neutral current and the effective weak mixing factor ' $s_{\nu q}^{2}$ ' in the $\nu_{\mu}-q$ scattering process. In terms of the two universal charge form factors $\bar{g}_{Z}^{2}(0)$ and $\bar{s}^{2}(t)$ they are given by

$$
\begin{aligned}
\rho_{\nu q} & =\frac{\bar{\rho}}{\left[1+\delta_{\text {c.c. }}\right]^{1 / 2}} \frac{1+\frac{\langle-t\rangle_{\text {c.c. }}}{m_{W}^{W}}}{1+\frac{\left\langle-t t_{\text {n.c. }}\right.}{m_{Z}^{2}}}+\frac{\hat{g}_{Z}^{2}}{8 \pi^{2}} a_{Z}, \\
s_{\nu q}^{2}(t) & =\bar{s}^{2}(t)+\frac{\hat{e}^{2}}{16 \pi^{2}} \bar{J}_{W}\left(t ; m_{\mu}\right)-\frac{\hat{e}^{2}}{8 \pi^{2} \hat{c}^{2}} a_{\gamma} .
\end{aligned}
$$

The box factors in eq. (3.60) are obtained from eq. (3.52):

$$
\begin{gathered}
\frac{\hat{g}_{Z}^{2}}{8 \pi^{2}} a_{Z}=\frac{\hat{g}_{Z}^{2}}{16 \pi^{2} \hat{c}^{2}}\left(\frac{5}{2}-\frac{15}{4} \hat{s}^{2}-\frac{1}{5} \hat{s}^{4}+\frac{14}{9} \hat{s}^{6}\right) \approx 0.0074, \\
\frac{\hat{e}^{2}}{8 \pi^{2} \hat{c}^{2}} a_{\gamma}=\frac{\hat{e}^{2}}{16 \pi^{2} \hat{c}^{4}}\left(\frac{5}{2}-\frac{61}{20} \hat{s}^{2}-\frac{9}{10} \hat{s}^{4}+\frac{14}{9} \hat{s}^{6}\right) \approx 0.0018,
\end{gathered}
$$

where $m_{W}^{2} / m_{Z}^{2}$ is replaced by $\hat{c}^{2}$ in order to reproduce the expressions in ref. [82]. With the estimates (3.53) and (3.56), we find

$$
\begin{aligned}
\rho_{\nu q} & \approx 0.9923 \bar{\rho}+0.0074, \\
s_{\nu q}^{2} & \approx 1.0295 \bar{s}^{2}(0)-0.0155 .
\end{aligned}
$$

These equations are useful in understanding qualitatively the effect of the $\nu_{\mu}-q$ scattering experiments off isoscalar targets, but we find that they give slightly inaccurate approximations to the quantities $q_{\alpha}$ (3.45).

In the following table, we compare the numerical predictions for the basic quantities $q_{\alpha}$ and the model-independent parameters of eq. (3.44) by using the exact matrix elements (3.45) and by using the approximation (3.57), for $\bar{g}_{Z}^{2}(0)=0.5492$ and $\bar{s}^{2}\left(-20 \mathrm{GeV}^{2}\right)=$ 0.2359 (the SM predictions for $m_{t}=175 \mathrm{GeV}$ and $m_{H}=100 \mathrm{GeV}$ ) :

\begin{tabular}{|l|r|r|}
\hline & eq. (3.45) & eq. (3.57) \\
& exact & approx. \\
\hline$u_{L}$ & 0.3435 & 0.3343 \\
$u_{R}$ & -0.1537 & -0.1537 \\
$d_{L}$ & -0.4260 & -0.4336 \\
$d_{R}$ & 0.0769 & 0.0769 \\
\hline$g_{L}^{2}$ & 0.2995 & 0.2998 \\
$g_{R}^{2}$ & 0.0295 & 0.0295 \\
$\delta_{L}^{2}$ & -0.0634 & -0.0763 \\
$\delta_{R}^{2}$ & 0.0177 & 0.0177 \\
\hline
\end{tabular}


It is clearly seen that the formulae (3.57), although reproducing $u_{L}$ and $d_{L}$ rather poorly, give, as expected, an excellent approximation for the most precisely measured parameter $g_{L}^{2}$. They give, however, a rather poor approximation for the parameter $\delta_{L}^{2}$ being off by $20 \%$, which is unsatisfactory in view of the experimental uncertainty (see section 4.2.1).

Fig. 10 illustrates the relation between the model-independent parameters $\left(g_{L}^{2}, g_{R}^{2}\right)$ and the two universal form factors $\left(\bar{s}^{2}(0), \bar{g}_{Z}^{2}(0)\right)$. The present data [78] (see section 4) constrain the 2-dimensional parameter space to the ellipse drawn in the same figure. The dashed line is the $\bar{\rho}=1\left(\bar{\delta}_{G}-\alpha T=0\right)$ curve $: \bar{g}_{Z}^{2}(0)=4 \sqrt{2} G_{F} m_{Z}^{2}=0.5486$. The thinness of the ellipse in the $\left(g_{L}^{2}, g_{R}^{2}\right)$ plane implies a strong correlation between $\bar{s}^{2}(0)$ and $\bar{g}_{Z}^{2}(0)$. It is worth noting that the effective charge $\bar{s}^{2}(0)$ derived from $\nu_{\mu}-q$ scattering experiments at $q^{2} \approx-20 \mathrm{GeV}^{2}$ is larger than the process-dependent effective mixing factor $s_{\nu q}^{2}$ by as much as 0.01: see eq. (3.62b).

\subsubsection{Neutral currents in $\nu_{\mu}-e$ scattering}

The total cross section for the processes $\nu_{\mu} e \rightarrow \nu_{\mu} e$ and $\bar{\nu}_{\mu} e \rightarrow \bar{\nu}_{\mu} e$ in terms of the helicity amplitudes $M_{L L}^{\nu_{\mu} e}$ and $M_{L R}^{\nu_{\mu} e}$ are given by

$$
\begin{aligned}
\sigma^{\nu e} & =\frac{m_{e} E_{\nu}}{4 \pi} \int_{0}^{1} d z\left\{\left|M_{L L}^{\nu_{\mu} e}\right|^{2}+(1-z)^{2}\left|M_{L R}^{\nu_{\mu} e}\right|^{2}\right\}, \\
\sigma^{\bar{\nu} e} & =\frac{m_{e} E_{\nu}}{4 \pi} \int_{0}^{1} d z\left\{(1-z)^{2}\left|M_{L L}^{\nu_{\mu} e}\right|^{2}+\left|M_{L R}^{\nu_{\mu} e}\right|^{2}\right\},
\end{aligned}
$$

where the variable $z$ is related to the momentum transfer $t$ by

$$
z=-t / t_{\max }, \quad t_{\max }=\frac{\left(s-m_{e}^{2}\right)^{2}}{s} \approx 2 m_{e} E_{\nu}
$$

with the approximation $s \equiv\left(p_{\nu}+p_{e}\right)^{2} \approx 2 m_{e} E_{\nu}$. The amplitudes in eq. (3.64) are obtained from eq. (3.43)

$$
\begin{aligned}
& M_{L L}^{\nu_{\mu} e}=-\frac{1}{2} \hat{e}^{2} \frac{\bar{\Gamma}_{2}^{\nu}(t)}{t}+\frac{1}{2}\left[-\frac{1}{2}+\bar{s}^{2}(t)\right] \frac{\bar{g}_{Z}^{2}(0)}{t-m_{Z}^{2}}+B_{L L}^{\nu_{\mu} e}(0,0), \\
& M_{L R}^{\nu_{\mu} e}=-\frac{1}{2} \hat{e}^{2} \frac{\bar{\Gamma}_{2}^{\nu}(t)}{t} \quad+\frac{1}{2} \bar{s}^{2}(t) \frac{\bar{g}_{Z}^{2}(0)}{t-m_{Z}^{2}}+B_{L R}^{\nu_{\mu} e}(0,0),
\end{aligned}
$$

where the $\nu_{\mu}$ charge radius factor $\bar{\Gamma}_{2}^{\nu}(t) / t$ is given by eq. (3.51) and the box form factors $B_{L L}^{\nu_{\mu} e}, B_{L R}^{\nu_{\mu} e}$ by

$$
\begin{array}{ll}
B_{L L}^{\nu_{\mu} e}(0,0)=\frac{\hat{g}^{4}}{16 \pi^{2} m_{W}^{2}} & +\frac{3 \hat{g}_{Z}^{4}}{16 \pi^{2} m_{Z}^{2}}\left(\frac{1}{2}\right)^{2}\left(-\frac{1}{2}+\hat{s}^{2}\right)^{2}, \\
B_{L R}^{\nu_{\mu} e}(0,0)= & -\frac{3 \hat{g}_{Z}^{4}}{16 \pi^{2} m_{Z}^{2}}\left(\frac{1}{2}\right)^{2}\left(\hat{s}^{2}\right)^{2},
\end{array}
$$


see eq. A.35). It is then straightforward to express the cross sections (3.64) in terms of the universal charge form factors $\bar{s}^{2}(0)$ and $\bar{g}_{Z}^{2}(0)$. Our results (3.66) and (3.67) agree with ref. 83.

As in the case of the $\nu_{\mu}-q$ scattering analysis it is useful to introduce the processdependent effective couplings $\rho_{\nu e}$ and $s_{\nu e}^{2}$ [24:

$$
\begin{aligned}
& M_{L L}^{\nu_{\mu} e}=2 \sqrt{2} G_{F} \rho_{\nu e}\left[\frac{1}{2}-s_{\nu e}^{2}(t)\right], \\
& M_{L R}^{\nu_{\mu} e}=2 \sqrt{2} G_{F} \rho_{\nu e}\left[-s_{\nu e}^{2}(t)\right] .
\end{aligned}
$$

From eqs. (3.66) and (3.68) one finds

$$
\begin{aligned}
\rho_{\nu e} & =\bar{\rho}+\frac{\hat{g}_{Z}^{2}}{16 \pi^{2}}\left[\frac{19}{4}-7 \hat{s}^{2}+6 \hat{s}^{4}\right], \\
s_{\nu e}^{2}(t) & =\bar{s}^{2}(t)+\frac{\hat{e}^{2}}{16 \pi^{2}} \bar{J}_{W}\left(t ; m_{\mu}\right)-\frac{\hat{g}_{Z}^{2} \hat{s}^{2}}{16 \pi^{2}}\left[\frac{19}{4}-\frac{17}{2} \hat{s}^{2}+6 \hat{s}^{4}\right],
\end{aligned}
$$

by neglecting higher order terms and by setting $m_{W}^{2} / m_{Z}^{2}=\hat{c}^{2}$. Here $\bar{\rho}$ and $\bar{J}_{W}\left(t ; m_{\mu}\right)$ are given by (3.55) and (3.51), respectively. The cross sections can then be expressed in terms of the model-independent parameters $\rho_{\nu e}$ and $s_{\nu e}^{2}$ by

$$
\begin{aligned}
& \frac{\sigma^{\nu e}}{E_{\nu}}=\frac{2 m_{e} G_{F}^{2}}{\pi} \rho_{\nu e}^{2} \int_{0}^{1} d z\left\{\left[\frac{1}{2}-s_{\nu e}^{2}(t)\right]^{2}+(1-z)^{2}\left[s_{\nu e}^{2}(t)\right]^{2}\right\}, \\
& \frac{\sigma^{\bar{\nu} e}}{E_{\nu}}=\frac{2 m_{e} G_{F}^{2}}{\pi} \rho_{\nu e}^{2} \int_{0}^{1} d z\left\{(1-z)^{2}\left[\frac{1}{2}-s_{\nu e}^{2}(t)\right]^{2}+\left[s_{\nu e}^{2}(t)\right]^{2}\right\},
\end{aligned}
$$

where $t=-2 m_{e} E_{\nu} z$ (3.65). For $E_{\nu}=25.7 \mathrm{GeV}$ (CHARM-II [84]), we find

$$
t_{\max }=2 m_{e} E_{\nu} \sim 2 m_{\mu}^{2} .
$$

In this momentum region the running of $\bar{s}^{2}(t)$ is negligible:

$$
\bar{s}^{2}(t)=\left\{\begin{array}{ll}
1.0072 \bar{s}^{2}(0)-0.0018 & \left(t=-m_{\mu}^{2}\right) \\
1.0080 \bar{s}^{2}(0)-0.0020 & \left(t=-2 m_{\mu}^{2}\right)
\end{array} .\right.
$$

Also the $\nu$-charge radius factor $\bar{J}_{W}\left(t ; m_{\mu}\right)$ has little $t$-dependence:

$$
\frac{\hat{e}^{2}}{16 \pi} \bar{J}_{W}\left(t ; m_{\mu}\right)=\left\{\begin{array}{ll}
-0.0061 & (t=0) \\
-0.0060 & \left(t=-m_{\mu}^{2}\right) \\
-0.0059 & \left(t=-2 m_{\mu}^{2}\right)
\end{array} .\right.
$$

Thus, the $t$-dependence of the effective mixing factor $s_{\nu e}^{2}(t)$ (3.69b) is negligibly small. From eqs. (3.69), (3.72) and (3.73) follows

$$
\begin{aligned}
\rho_{\nu e} & \approx \bar{\rho}+0.0121, \\
s_{\nu e}^{2}(0) \approx s_{\nu e}^{2}\left(-m_{\mu}^{2}\right) & \approx 1.0072 \bar{s}^{2}(0)-0.0103 .
\end{aligned}
$$


In the limit of negligible $t$-dependence of $s_{\nu e}^{2}$, eq. (3.70) becomes :

$$
\begin{aligned}
& \frac{\sigma^{\nu e}}{E_{\nu}}=\frac{2 m_{e} G_{F}^{2}}{\pi} \rho_{\nu e}^{2}\left[\left(\frac{1}{2}-s_{\nu e}^{2}\right)^{2}+\frac{1}{3}\left(s_{\nu e}^{2}\right)^{2}\right], \\
& \frac{\sigma^{\bar{\nu} e}}{E_{\nu}}=\frac{2 m_{e} G_{F}^{2}}{\pi} \rho_{\nu e}^{2}\left[\frac{1}{3}\left(\frac{1}{2}-s_{\nu e}^{2}\right)^{2}+\left(s_{\nu e}^{2}\right)^{2}\right],
\end{aligned}
$$

with $s_{\nu e}^{2}=s_{\nu e}^{2}(0)$. This is the form entering the analysis of ref. [79]: they combined the three experiments [84] and expressed the result in terms of the model-independent parameters $\rho_{\nu e}$ and $s_{\nu e}^{2}(3.40)$. In our analysis the above parametrization (3.75) is used to reproduce the combined measured cross sections from the fit [79] in terms of $\rho_{\nu e}$ and $s_{\nu e}^{2}$. These cross sections are then analysed in our framework by using the defining equation (3.64).

Fig. 11 illustrates the constraint by the data similarly to Fig. 10. The approximation (3.74) is found to reproduce our results accurately. The dashed line denotes the curve $\bar{\rho}=1\left(\bar{\delta}_{G}-\alpha T=0\right)$. The ratio of the $\nu_{\mu} e$ and $\bar{\nu}_{\mu} e$ cross sections is measured accurately, and hence the form factor $\bar{s}^{2}(0)$ is constrained fairly independently of $\bar{g}_{Z}^{2}(0)$ from the $\nu_{\mu}-e$ scattering experiments.

\subsubsection{Neutral currents in $e-q$ interactions}

The effective Lagrangian of the parity-violating $e-q$ interaction [24]

$$
\mathcal{L}_{P V}=-\frac{G_{F}}{\sqrt{2}} \sum_{q}\left[C_{1 q} \bar{\psi}_{e} \gamma^{\mu} \gamma_{5} \psi_{e} \cdot \bar{\psi}_{q} \gamma_{\mu} \psi_{q}+C_{2 q} \bar{\psi}_{e} \gamma^{\mu} \psi_{e} \cdot \bar{\psi}_{q} \gamma_{\mu} \gamma_{5} \psi_{q}\right]
$$

can be rewritten in terms of left- and right-handed currents as follows :

$$
\mathcal{L}_{P V}=-\frac{G_{F}}{\sqrt{2}} \sum_{q}\left[C_{1 q}\left(J_{R}^{e}-J_{L}^{e}\right) \cdot\left(J_{R}^{q}+J_{L}^{q}\right)+C_{2 q}\left(J_{R}^{e}+J_{L}^{e}\right) \cdot\left(J_{R}^{q}-J_{L}^{q}\right)\right] .
$$

The effective couplings $C_{1 q}, C_{2 q}$ expressed in terms of the helicity amplitudes read :

$$
\begin{aligned}
& C_{1 q}=C_{1 q}^{M}+C_{1 q}^{(\gamma)}=\frac{1}{2 \sqrt{2} G_{F}}\left[M_{L L}^{e q}-M_{R L}^{e q}+M_{L R}^{e q}-M_{R R}^{e q}\right]+C_{1 q}^{(\gamma)}, \\
& C_{2 q}=C_{2 q}^{M}+C_{2 q}^{(\gamma)}=\frac{1}{2 \sqrt{2} G_{F}}\left[M_{L L}^{e q}-M_{L R}^{e q}+M_{R L}^{e q}-M_{R R}^{e q}\right]+C_{2 q}^{(\gamma)} .
\end{aligned}
$$

Here $C_{1 q}^{(\gamma)}$ and $C_{2 q}^{(\gamma)}$ denote the sum of the contributions from the photonic correction to the axial vector $Z e e$ vertex and the $Z \gamma$ box correction [85], which are not included in our helicity amplitudes (3.43). They are found in refs. 855, 86, :

$$
\begin{aligned}
& \left(4 \sqrt{2} G_{F} m_{Z}^{2}\right) C_{1 q}^{(\gamma)}=\frac{\hat{g}_{Z}^{2} \hat{e}^{2}}{16 \pi^{2}}\left\{-2\left(I_{3 q}-2 Q_{q} \hat{s}^{2}\right)+6 I_{3 q} Q_{q}\left(1-4 \hat{s}^{2}\right)\left(\ln \frac{m_{Z}^{2}}{M^{2}}+\frac{3}{2}\right)\right\},(3 . \\
& \left(4 \sqrt{2} G_{F} m_{Z}^{2}\right) C_{2 q}^{(\gamma)}=\frac{\hat{g}_{Z}^{2} \hat{e}^{2}}{16 \pi^{2}}\left\{-2 I_{3 q} Q_{q}^{2}\left(1-4 \hat{s}^{2}\right)+6 Q_{q}\left(I_{3 q}-2 Q_{q} \hat{s}^{2}\right)\left(\ln \frac{m_{Z}^{2}}{M^{2}}+\frac{3}{2}\right)\right\} .
\end{aligned}
$$


$M^{2} \approx\langle-t\rangle \approx 1.5 \mathrm{GeV}^{2}$ is used in the analysis of the SLAC $e D$ scattering experiments 87. By inserting eq. (3.43) into $C_{1 q}^{M}$ and $C_{2 q}^{M}$ defined above, one finds

$$
\begin{aligned}
2 \sqrt{2} G_{F} \cdot C_{1 q}^{M}= & \frac{\hat{e}^{2}}{t}\left(-Q_{q}\right)\left[2\left(\Gamma_{1}^{e_{L}}-\Gamma_{1}^{e_{R}}\right)+\bar{\Gamma}_{2}^{e_{L}}\right](t)+\frac{\bar{g}_{Z}^{2}(0)}{t-m_{Z}^{2}}\left(-\frac{1}{2}\right)\left(I_{3 q}-2 Q_{q} \bar{s}^{2}(t)\right) \\
& +B_{L L}^{e q}-B_{R L}^{e q}+B_{L R}^{e q}-B_{R R}^{e q} \\
2 \sqrt{2} G_{F} \cdot C_{2 q}^{M}= & \frac{\hat{e}^{2}}{t}\left[\left(-Q_{q}\right) 2\left(\Gamma_{1}^{q_{L}}-\Gamma_{1}^{q_{R}}\right)-2 I_{3 q} \bar{\Gamma}_{2}^{q_{L}}\right](t)+\frac{\bar{g}_{Z}^{2}(0)}{t-m_{Z}^{2}}\left(-\frac{1}{2}+2 \bar{s}^{2}(t)\right)\left(I_{3 q}\right) \\
& +B_{L L}^{e q}-B_{L R}^{e q}+B_{R L}^{e q}-B_{R R}^{e q} .
\end{aligned}
$$

By adopting the SM predictions for the vertex and box form factors of appendix A, the model-independent parameters $C_{i q}$ of the low energy effective Lagrangian (3.76) are readily evaluated as functions of $\bar{s}^{2}(t)$ and $\bar{g}_{Z}^{2}(0)$. More explicitly, one finds

$$
\begin{aligned}
4 & \sqrt{2} G_{F} m_{Z}^{2} C_{1 q}^{M} \\
= & \frac{\bar{g}_{Z}^{2}(0)}{1-t / m_{Z}^{2}}\left[I_{3 q}-2 Q_{q} \bar{s}^{2}(t)\right]+\frac{\hat{g}_{Z}^{2} \hat{e}^{2}}{16 \pi^{2}} Q_{q}\left[\left(1-4 \hat{s}^{2}\right) J_{Z}+2\left(J_{W}-\bar{J}_{W}\right)\right] \\
& +\frac{\hat{g}_{Z}^{4}}{16 \pi^{2}}\left\{\frac{3}{4} I_{3 q}\left(I_{3 q}-2 Q_{q} \hat{s}^{2}\right)\left[1+\left(1-4 \hat{s}^{2}\right)^{2}\right]+2 \hat{c}^{2}(q=u)-\frac{\hat{c}^{2}}{2}(q=d)\right\}, \quad \text { (3.81a) } \\
4 & \sqrt{2} G_{F} m_{Z}^{2} C_{2 q}^{M} \\
= & \left.\frac{\bar{g}_{Z}^{2}(0)}{1-t / m_{Z}^{2}} I_{3 q}\left[1-4 \bar{s}^{2}(t)\right]+\frac{\hat{g}_{Z}^{2} \hat{e}^{2}}{16 \pi^{2}}\left[2 I_{3 q} Q_{q}\left(2 I_{3 q}-4 Q_{q} \hat{s}^{2}\right) J_{Z}+2 Q_{q} J_{W}-4 I_{3 q} \bar{J}_{W}\right)\right] \\
& +\frac{\hat{g}_{Z}^{4}}{16 \pi^{2}}\left\{\frac{3}{2}\left(1-4 \hat{s}^{2}\right)\left[\left(I_{3 q}-Q_{q} \hat{s}^{2}\right)^{2}+\left(Q_{q} \hat{s}^{2}\right)^{2}\right]+2 \hat{c}^{2}(q=u)-\frac{\hat{c}^{2}}{2}(q=d)\right\}, \quad(3.81 \mathrm{~b})
\end{aligned}
$$

where the factors $J_{Z} \equiv J_{Z}\left(t ; m_{e}\right), J_{W} \equiv J_{W}\left(t ; m_{\nu_{e}}\right)$ and $\bar{J}_{W} \equiv \bar{J}_{W}\left(t ; m_{\nu_{e}}\right)$ are given in appendix A. The sum of (3.81) and (3.79) agrees with ref. 85.

At $t=-1.5 \mathrm{GeV}^{2}, \bar{s}^{2}(t)$ is calculated from $\bar{s}^{2}(0)$ as

$$
\bar{s}^{2}(t) \approx 1.0183 \bar{s}^{2}(0)-0.0058,
$$

and the numerical values for $J_{Z}, J_{W}$ and $\bar{J}_{W}$ are $J_{Z} \approx-6.97, J_{W} \approx-6.80, \bar{J}_{W} \approx-7.69$. The non-universal (vertex and box) corrections for $C_{i q}$ are estimated numerically as

$$
\begin{aligned}
& C_{1 u} \approx\left[C_{1 u}^{M}\right]_{\mathrm{IBA}}+0.0061+0.0007, \\
& C_{1 d} \approx\left[C_{1 d}^{M}\right]_{\mathrm{IBA}}-0.0011+0.0009, \\
& C_{2 u} \approx\left[C_{2 u}^{M}\right]_{\mathrm{IBA}}+0.0082+0.0048, \\
& C_{2 d} \approx\left[C_{2 d}^{M}\right]_{\mathrm{IBA}}-0.0070+0.0043,
\end{aligned}
$$

where the second terms in the r.h.s. denote the electroweak vertex/box corrections for $C_{i q}^{M}$, and the last terms denote the external photonic corrections, $C_{i q}^{(\gamma)}$. The improved 
Born expressions $\left[C_{i q}^{M}\right]_{\text {IBA }}$ 's can be expressed by

$$
\begin{aligned}
{\left[C_{1 q}^{M}\right]_{\mathrm{IBA}} } & =\frac{\bar{\rho}}{1-t / m_{Z}^{2}}\left[I_{3 q}-2 Q_{q} \bar{s}^{2}(t)\right], \\
{\left[C_{2 q}^{M}\right]_{\mathrm{IBA}} } & =\frac{\bar{\rho}}{1-t / m_{Z}^{2}} I_{3 q}\left[1-4 \bar{s}^{2}(t)\right],
\end{aligned}
$$

with $\bar{\rho} \equiv \bar{g}_{Z}^{2}(0) /\left(4 \sqrt{2} G_{F} m_{Z}^{2}\right)$ as in eq. (3.55).

In the polarized $e D$ experiment only the combinations $2 C_{1 u}-C_{1 d}$ and $2 C_{2 u}-C_{2 d}$ [24] are well measured. A model-independent determination of these two combinations is performed in section 4. Fig. 12 shows the relation between the model-independent parameters $\left(2 C_{1 u}-C_{1 d}, 2 C_{2 u}-C_{2 d}\right)$ and the two universal parameters $\left(\bar{s}^{2}(0), \bar{g}_{Z}^{2}(0)\right)$, together with the 1- $\sigma$ contour of the result of the analysis obtained in section 4 from the experimental data [87. Note that the vertex and box corrections (especially the $W W$ box contributions) in eqs. (3.80) are important in these combinations yielding :

$$
\begin{aligned}
& 2 C_{1 u}-C_{1 d} \approx\left[2 C_{1 u}^{M}-C_{1 d}^{M}\right]_{\mathrm{IBA}}+0.0134+0.0005, \\
& 2 C_{2 u}-C_{2 d} \approx\left[2 C_{2 u}^{M}-C_{2 d}^{M}\right]_{\mathrm{IBA}}+0.0234+0.0052 .
\end{aligned}
$$

As before, the second terms denote the vertex/box corrections in $C_{i q}^{M}$, while the last terms denote contributions from $C_{i q}^{\gamma}$. The majority of the non-universal contributions above come from the $W W$ box diagram. Since the typical contribution of the improved Born approximation to these factors are $\left[2 C_{1 u}^{M}-C_{1 d}^{M}\right]_{\mathrm{IBA}} \approx 0.7089$ and $\left[2 C_{2 u}^{M}-C_{2 d}^{M}\right]_{\mathrm{IBA}} \approx 0.0751$ for $\bar{g}_{Z}^{2}(0)=0.5492$ and $\bar{s}^{2}\left(-1.5 \mathrm{GeV}^{2}\right)=0.2375$ (the SM predictions for $m_{t}=175 \mathrm{GeV}$ and $m_{H}=100 \mathrm{GeV}$ ), the non-propagator correction terms are appreciable in these observables.

In the case of atomic parity violation the momentum transfer is so small that the matrix elements for nucleons should be calculated. Marciano and Sirlin [85] introduced effective couplings $C_{1 p}$ and $C_{1 n}$ for nucleons, which may be separated as in eq. (3.78)

$$
\begin{aligned}
& C_{1 p}=C_{1 p}^{M}+C_{1 p}^{(\gamma)}, \\
& C_{1 n}=C_{1 n}^{M}+C_{1 n}^{(\gamma)} .
\end{aligned}
$$

Here $C_{1 p}^{M}$ and $C_{1 n}^{M}$ are the contributions from the neutral current amplitudes (3.43), which can be expressed in terms of $C_{1 u}^{M}$ and $C_{1 d}^{M}$ by

$$
\begin{aligned}
& C_{1 p}^{M}=2 C_{1 u}^{M}+C_{1 d}^{M}, \\
& C_{1 n}^{M}=C_{1 u}^{M}+2 C_{1 d}^{M},
\end{aligned}
$$

or more explicitly,

$$
\left(4 \sqrt{2} G_{F} m_{Z}^{2}\right) C_{1 p}^{M}=\bar{g}_{Z}^{2}(0)\left[\frac{1}{2}-2 \bar{s}^{2}(0)\right]+\frac{\hat{g}_{Z}^{2} \hat{e}^{2}}{16 \pi^{2}}\left\{\left(1-4 \hat{s}^{2}\right) J_{Z}+2\left(J_{W}-\bar{J}_{W}\right)\right\}
$$




$$
\begin{gathered}
+\frac{\hat{g}_{Z}^{4}}{16 \pi^{2}}\left\{\frac{9}{16}\left(1-\frac{20}{9} \hat{s}^{2}\right)\left[1+\left(1-4 \hat{s}^{2}\right)^{2}\right]+\frac{7}{2} \hat{c}^{2}\right\} \\
\left(4 \sqrt{2} G_{F} m_{Z}^{2}\right) C_{1 n}^{M}=\bar{g}_{Z}^{2}(0)\left[-\frac{1}{2}\right]+\frac{\hat{g}_{Z}^{4}}{16 \pi^{2}}\left\{\frac{9}{16}\left(1-\frac{16}{9} \hat{s}^{2}\right)\left[1+\left(1-4 \hat{s}^{2}\right)^{2}\right]+\hat{c}^{2}\right\}
\end{gathered}
$$

with

$$
\begin{aligned}
& J_{Z}=\frac{2}{3} \ln \frac{m_{e}^{2}}{m_{Z}^{2}}-\frac{1}{9}, \\
& J_{W}-\bar{J}_{W}=\frac{8}{9},
\end{aligned}
$$

which can be obtained from eqs. (A.27) by taking the $q^{2} \rightarrow 0$ limit. $C_{1 p}^{(\gamma)}$ and $C_{1 n}^{(\gamma)}$ are the contributions from the photonic correction to the axial vector $Z e e$ vertex and the $Z \gamma$ box correction 85:

$$
\begin{aligned}
& 4 \sqrt{2} G_{F} m_{Z}^{2} C_{1 p}^{(\gamma)}=\frac{\hat{g}_{Z}^{2} \hat{e}^{2}}{16 \pi^{2}}\left\{-\left(1-4 \hat{s}^{2}\right)^{2}+5\left(1-4 \hat{s}^{2}\right)\left[K+\frac{4}{5}\left(\xi_{1}\right)_{B}^{p}\right]\right\} \\
& 4 \sqrt{2} G_{F} m_{Z}^{2} C_{1 n}^{(\gamma)}=\frac{\hat{g}_{Z}^{2} \hat{e}^{2}}{16 \pi^{2}}\left\{-\left(1-4 \hat{s}^{2}\right)^{2}+4\left(1-4 \hat{s}^{2}\right)\left[K+\left(\xi_{1}\right)_{B}^{n}\right]\right\}
\end{aligned}
$$

The last terms on the right-hand sides of eqs. (3.90) denote the $\gamma Z$-box corrections which are sensitive to the nucleon structure. The constants $K,\left(\xi_{1}\right)_{B}^{p}$ and $\left(\xi_{1}\right)_{B}^{n}$ have been estimated in ref. [85] to be

$$
K=9.6 \pm 1,(\xi)_{B}^{p}=2.55,(\xi)_{B}^{n}=1.74
$$

By estimating numerically the vertex/box corrections in eqs. (3.88) and (3.90), we find

$$
\begin{aligned}
& C_{1 p} \approx\left[C_{1 p}^{M}\right]_{\mathrm{IBA}}+0.0107+0.0027 \\
& C_{1 n} \approx\left[C_{1 n}^{M}\right]_{\mathrm{IBA}}+0.0038+0.0023
\end{aligned}
$$

where the second terms denote the weak vertex/box corrections for $C_{1 p}^{M}$ and $C_{1 n}^{M}$, and the last terms denote the photonic corrections, $C_{1 p}^{(\gamma)}$ and $C_{1 n}^{(\gamma)}$. The improved Born approximations $\left[C_{1 p}^{M}\right]_{\mathrm{IBA}}$ and $\left[C_{1 n}^{M}\right]_{\mathrm{IBA}}$ are given simply by

$$
\begin{aligned}
{\left[C_{1 p}^{M}\right]_{\mathrm{IBA}} } & =\bar{\rho}\left[\frac{1}{2}-2 \bar{s}^{2}(0)\right], \\
{\left[C_{1 n}^{M}\right]_{\mathrm{IBA}} } & =\bar{\rho}\left[-\frac{1}{2}\right] .
\end{aligned}
$$

Their typical numerical values are found to be $\left[C_{1 p}^{M}\right]_{\mathrm{IBA}} \approx 0.0223$ and $\left[C_{1 n}^{M}\right]_{\mathrm{IBA}} \approx-0.5005$, for $\bar{g}_{Z}^{2}(0)=0.5492$ and $\bar{s}^{2}(0)=0.2389$ (the SM predictions for $m_{t}=175 \mathrm{GeV}$ and $\left.m_{H}=100 \mathrm{GeV}\right)$. Note that the non-universal corrections are important especially for 
$C_{1 p}$, where the effect comes mainly from the $W W$ box contribution, or the term with the factor $\frac{7}{2} \hat{c}^{2}$ in eq. (3.88a).

The weak charge $Q_{W}(A, Z)$ of an atom is given in terms of $C_{1 p}$ an $C_{1 n}$ by

$$
Q_{W}(A, Z)=2(A-Z) C_{1 n}+2 Z C_{1 p}
$$

which in the case of cesium is

$$
Q_{W}\left({ }_{55}^{133} \mathrm{Cs}\right)=156 C_{1 n}+110 C_{1 p} .
$$

Numerically they are estimated as

$$
Q_{W}(\mathrm{Cs}) \approx \bar{g}_{Z}^{2}(0)\left[-41.92-400.99 \bar{s}^{2}(0)\right]+1.77+0.65 .
$$

where the first term comes from the IBA approximation to $C_{1 p}^{M}$ and $C_{1 n}^{M}(3.93)$, the second term comes from the electroweak vertex/box contributions to them, and the last term from the external photonic corrections of eq. (3.90). It is clear from the above result that the vertex and box corrections should be carefully taken account of in extracting the electroweak parameters from the $Q_{W}$ measurements.

In Fig. 13, the parameter $Q_{W}(\mathrm{Cs})$ of eq. (3.95) is shown as a function of the two universal parameters $\left(\bar{s}^{2}(0), \bar{g}_{Z}^{2}(0)\right)$ in the range $0.20<\bar{s}^{2}(0)<0.26$ and $0.52<\bar{g}_{Z}^{2}(0)<$ 0.57 together with the $1-\sigma$ contour of the data 80] (dashed lines). The horizontal straight dashed line denotes the line $\bar{\rho}=1\left(\bar{\delta}_{G}-\alpha T=0\right)$. It is worth noting that the correlation in the $\bar{s}^{2}(0)$ and $\bar{g}_{Z}^{2}(0)$ form factors obtained from the cesium weak charge $Q_{W}(133,55)$ in the figure is opposite to that obtained from the $\nu_{\mu}-q$ scattering experiments (see Fig. 10). The cesium $Q_{W}$ measurement implies an anti-correlation between $\bar{s}^{2}(0)$ and $\bar{g}_{Z}^{2}(0)$, or $\bar{\rho}$. This is opposite to the trend observed for the constraints from the $\nu_{\mu}-q$ experiments. For further discussion, see section 4.

\subsection{Charged current experiments}

In the charged current sector we consider two precision experiments: the muon lifetime [25] and the $W$ boson mass measurements [25, 88].

From the matrix element (2.6) one finds for the muon decay constant

$$
G_{F}=\frac{\bar{g}_{W}^{2}(0)+\hat{g}^{2} \bar{\delta}_{G}}{4 \sqrt{2} m_{W}^{2}},
$$

where the factor $\bar{\delta}_{G}$ denotes the sum of the vertex and the box contributions. It has been calculated within the SM in ref. [50] :

$$
\begin{aligned}
\bar{\delta}_{G} & =\frac{\hat{g}^{2}}{8 \pi^{2}}\left[1+\left(\frac{1}{4 \hat{s}^{2}}-1\right) \ln \frac{1}{\hat{c}^{2}}\right], \\
& \approx 0.0055,
\end{aligned}
$$


where the pinch term 34 has been subtracted as explained in section 2: see eqs. (2.27) and (2.28).

The expression (3.97) enables one to predict the physical $W$ mass in terms of the charge form factor $\bar{g}_{W}^{2}(0)$. Numerically, one finds :

$$
\begin{aligned}
m_{W}^{2} & =\frac{\bar{g}_{W}^{2}(0)+\hat{g}^{2} \bar{\delta}_{G}}{4 \sqrt{2} G_{F}} \\
& \approx\left[15155.9 \bar{g}_{W}^{2}(0)+46.7 \frac{\bar{\delta}_{G}-0.0055}{\alpha}+35.2\right] \mathrm{GeV}^{2}
\end{aligned}
$$

Once the numerical value of $\bar{\delta}_{G}$ factor is known, the measurement of the $m_{W}$ mass determines directly the charge form factor $\bar{g}_{W}^{2}(0)$.

The form factor $\bar{g}_{W}^{2}(0)$ can be calculated in terms of the $S, T$ and $U$ parameters in the $\mathrm{SU}(2)_{\mathrm{L}} \times \mathrm{U}(1)_{\mathrm{Y}}$ models. Insertion of the expansion $(2.38 \mathrm{c})$ leads to

$$
m_{W}(\mathrm{GeV})=79.840-0.291 S+0.417 T+0.332 U-0.136 \delta_{\alpha}
$$

in excellent agreement with eq. (3.99) for $\bar{\delta}_{G}=0.0055$. The prediction for a different $\bar{\delta}_{G}$ value follows from the above expression by simply making the substitution (2.39).

Fig. 14 shows the SM predictions for $m_{W}$ in the plane of $m_{t}$ and $m_{H}$, for $\delta_{\alpha}=0$ and $\bar{\delta}_{G}=0.0055$. In the $\mathrm{O}\left(\alpha \alpha_{s}\right)$ corrections to the SM contributions to the $S, T, U$ parameters $\alpha_{s}\left(m_{Z}\right)$ is set to 0.12 . Changing $\alpha_{s}\left(m_{Z}\right)$ by \pm 0.01 affects the prediction of $m_{W}$ by about $\mp 0.004 \mathrm{GeV}$. The mean and standard deviation of the present $m_{W}$ measurement (see section 4.3) are indicated by dashed lines.

Note that among the electroweak observables examined in this paper, only $m_{W}$ is sensitive to the $U$ parameter. Hence, when performing a general fit to the $S, T, U$ parameters, the mean $(\langle U\rangle)$ and standard deviation $(\Delta U)$ of the $U$ parameter are determined solely by the mean $\left(\left\langle m_{W}\right\rangle\right)$ and standard deviation $\left(\Delta m_{W}\right)$ of $m_{W}$ :

$$
\begin{aligned}
& \langle U\rangle=\left[\left\langle m_{W}(\mathrm{GeV})\right\rangle-79.840+0.291\langle S\rangle-0.417\langle T\rangle+0.136 \delta_{\alpha}\right] / 0.332,(3.101 \mathrm{a}) \\
& \Delta U \approx \Delta m_{W}(\mathrm{GeV}) / 0.332 .
\end{aligned}
$$

Here $\langle S\rangle$ and $\langle T\rangle$ denote the best-fit values from other experiments. The present experimental error of $\Delta m_{W}=0.16 \mathrm{GeV}$ induces $\Delta U=0.48$, while $\Delta m_{W}=0.05 \mathrm{GeV}$, the precision anticipated in future LEP200 experiments, would give $\Delta U=0.15$. The full error $\Delta U$ should be slightly larger than the above estimate, since $S$ and $T$ were fixed and set at their best values in deriving (3.101b). 


\section{Experimental data and the electroweak parameters}

Based on the formalism introduced in the previous sections the values for the form factors are inferred from fits to the data of electroweak precision experiments : $\bar{g}_{Z}^{2}\left(m_{Z}^{2}\right)$, $\bar{s}^{2}\left(m_{Z}^{2}\right), \bar{\delta}_{b}\left(m_{Z}^{2}\right)$ from the LEP/SLC experiments on the $Z$-pole, $\bar{g}_{Z}^{2}(0), \bar{s}^{2}(0)$ from the low energy neutral current experiments at $q^{2} \approx 0$, and $\bar{g}_{W}^{2}(0)$ from the $W$ mass measurements at $p \bar{p}$ colliders.

\section{1 $Z$ boson parameters}

The analysis is based on the data from the LEP and SLC experiments published up to the year 1993 [26, 89, 90]. Discussions of the recent update from LEP [91] and the precision measurement of the left-right asymmetry at SLC [31] are postponed to section 6 .

The $Z$ line-shape parameters resulting from a combined fit performed by the LEP electroweak group [26] are :

$$
\begin{array}{ll}
m_{Z}(\mathrm{GeV}) & =91.187 \pm 0.007 \\
\Gamma_{Z}(\mathrm{GeV}) & =2.489 \pm 0.007 \\
\sigma_{h}^{0}(\mathrm{nb}) & =41.56 \pm 0.14 \\
R_{\ell}=\sigma_{h}^{0} / \sigma_{\ell}^{0} & =20.763 \pm 0.049 \\
A_{\mathrm{FB}}^{0, \ell} & =0.0158 \pm 0.0018
\end{array} \quad \rho_{\text {corr }}=\left(\begin{array}{rrrrr}
1 & -0.157 & 0.017 & 0.012 & 0.075 \\
& 1 & -0.070 & 0.003 & 0.006 \\
& 1 & 0.137 & 0.003 \\
& & 1 & 0.008 \\
& & & & 1
\end{array}\right) .
$$

The other electroweak data used in our fit are [26, 89]:

$$
\begin{aligned}
P_{\tau} & =-0.139 \pm 0.014, \\
A_{\mathrm{LR}}^{0} & =0.10 \pm 0.044 \quad(\mathrm{SLD}[90]), \\
A_{\mathrm{FB}}^{0, b} & =0.099 \pm 0.006 \\
A_{\mathrm{FB}}^{0, c} & =0.075 \pm 0.015 \\
R_{b}=\sigma_{b}^{0} / \sigma_{h}^{0} & =0.2203 \pm 0.0027 \quad(\mathrm{LEP}+\mathrm{SLD}) .
\end{aligned}
$$

Definitions of all the above observables and their theoretical expressions have been given in section 3.1.

The $Z$ mass, $m_{Z}=91.187 \mathrm{GeV}$, is treated as an input parameter neglecting its error. This is justified because of the smallness of the experimental uncertainty and correlations. For the fits to be described below a few general conditions are anticipated : (a) only three neutrinos $\left(N_{\nu}=3\right)$ contribute to the invisible width of $Z$, (b) the perturbative QCD corrections with the finite quark mass effects are taken as given explicitly in section 3.1, (c) the vertex and box corrections are calculated in the SM and given in Table 3 and \&, (d) the $Z b_{L} b_{L}$ vertex is taken into account by the quantity $\bar{\delta}_{b}\left(m_{Z}^{2}\right)$, which is treated in the fit as a free parameter just as the universal parameters $\bar{g}_{Z}^{2}\left(m_{Z}^{2}\right)$ and $\bar{s}^{2}\left(m_{Z}^{2}\right)$. 
Various methods to determine the QCD coupling constant have led to consistent results with a typical uncertainty of $\Delta \alpha_{s}\left(m_{Z}\right) \approx 0.01$. However, this is far from making it precise enough to be used as a fixed input parameter, since the fitted electroweak parameters are found to be rather sensitive to the assumed value of $\alpha_{s}\left(m_{Z}\right)$ : see, for instance, eq. (4.3) below. For this reason, and also for the convenience of GUT studies, $\alpha_{s} \equiv \alpha_{s}\left(m_{Z}\right)_{\overline{\mathrm{MS}}}$ is treated throughout our fits as an external input parameter and, consequently, the best-fit values of the fit parameters and the minimum $\chi^{2}$ are always presented as functions of $\alpha_{s}$. Once a precise determination of $\alpha_{s}$ from independent data is available, it is straightforward to get the correspondingly adjusted best-fit values without repeating the fit. It is also easy to infer from our results the quantitative consequences of a particular GUT model predicting the relationship between $\alpha_{s}$ and $\sin ^{2} \theta_{W}\left(m_{Z}\right) \overline{\mathrm{MS}}$.

The overall fit to all $Z$ parameters listed above gives the following result :

$$
\begin{aligned}
\bar{g}_{Z}^{2}\left(m_{Z}^{2}\right)= & 0.5542-0.00030 \frac{\alpha_{s}-0.12}{0.01} \pm 0.0017 \\
\bar{s}^{2}\left(m_{Z}^{2}\right)= & 0.2313+0.00008 \frac{\alpha_{s}-0.12}{0.01} \pm 0.0007 \\
\bar{\delta}_{b}\left(m_{Z}^{2}\right)=- & 0.0061-0.00430 \frac{\alpha_{s}-0.12}{0.01} \pm 0.0034 \\
& \chi_{\min }^{2}=1.53+\left(\frac{\alpha_{s}-0.1029}{0.0128}\right)^{2},
\end{aligned}
$$

where the errors and correlations are nearly independent of $\alpha_{s}$. The above parametrization for the $\alpha_{s}$ dependences of the mean values and $\chi_{\min }^{2}$ are accurate interpolations of our fit results (at the level of 1\%) in the range $0.09<\alpha_{s}<0.15$. The bottom and charm quark masses were set to $m_{b}=4.7 \mathrm{GeV}$ and $m_{c}=1.4 \mathrm{GeV}$. A shift of the bottom mass by $\pm 0.2 \mathrm{GeV}$ implies only the fitted $\bar{\delta}_{b}\left(m_{Z}^{2}\right)$ value to be displaced by \pm 0.0002 , which is negligibly small compared to its error $( \pm 0.0034)$. Similarly, shifting the charm quark mass by $\pm 0.2 \mathrm{GeV}$ does not affect the above results, as expected. In particular, in the favored range $0.11 \lesssim \alpha_{s}\left(m_{Z}\right) \lesssim 0.13$ the quality of the fit is good, e.g. $\chi_{\min }^{2}=4.6$ at $\alpha_{s}=0.12$ for $9-3=6$ degrees of freedom.

Results of the fit (4.3) are displayed in Fig. 15 by the $1-\sigma$ allowed contours in the three projections, $\left(\bar{s}^{2}\left(m_{Z}^{2}\right), \bar{g}_{Z}^{2}\left(m_{Z}^{2}\right)\right),\left(\bar{s}^{2}\left(m_{Z}^{2}\right), \bar{\delta}_{b}\left(m_{Z}^{2}\right)\right)$, and $\left(\bar{\delta}_{b}\left(m_{Z}^{2}\right), \bar{g}_{Z}^{2}\left(m_{Z}^{2}\right)\right)$. The contours are shown for three representative $\alpha_{s}$-values : $\alpha_{s}=0.11$ (dashed), 0.12 (solid) and 0.13 (dashdotted). Also shown by the lattices are the SM predictions for $m_{t}=(100-200) \mathrm{GeV}$ and $m_{H}=(50-1000) \mathrm{GeV}$. In these SM predictions, all known two-loop corrections of the $O\left(m_{t}^{4}\right)$ and $O\left(\alpha \alpha_{s}\right)$ level [46, 47, 54 58, 63] are included, as explained in detail in appendix C. Hence, the predictions depend weakly on $\alpha_{s}$ due to the $O\left(\alpha \alpha_{s}\right)$ corrections, as well as on $\bar{\delta}_{G}$ and $\delta_{\alpha}$ which are needed to predict the charge form factors from the known $\left(\alpha, G_{F}, m_{Z}\right)$ values. The SM predictions in Fig. 15 are calculated for $\alpha_{s}=0.12, \bar{\delta}_{G}=0.0055$ and $\delta_{\alpha}=0$. Changing $\alpha_{s}$ by \pm 0.01 has little effect, but changing $\delta_{\alpha}$ by \pm 0.10 leads to 
a shift in the SM predictions for $\bar{s}^{2}\left(m_{Z}^{2}\right)$ by $\mp 0.00026$, which is as large as $40 \%$ of its uncertainty: see eq. (4.3).

It is clearly seen from the figure and eq. (4.3) that the weak mixing form factor $\bar{s}^{2}\left(m_{Z}^{2}\right)$ is determined almost independently of $\alpha_{s}$, while the $Z$ coupling strength $\bar{g}_{Z}^{2}\left(m_{Z}^{2}\right)$ is anti-correlated with the assumed $\alpha_{s}$ value as a reflection of its sensitivity to the total $Z$ width. This anti-correlation leads in the SM to a preference of larger $m_{t}$ for smaller $\alpha_{s}$, since $\bar{g}_{Z}^{2}\left(m_{Z}^{2}\right)$ grows with $m_{t}$ (see Fig. 2). Furthermore, the fitted $\bar{\delta}_{b}\left(m_{Z}^{2}\right)$ value depends strongly on the $\alpha_{s}$ value assumed. The minimum of $\chi^{2}$ is reached at $\alpha_{s}=0.1029$ in eq. (4.3b), a value slightly outside the range $0.11 \lesssim \alpha_{s}\left(m_{Z}\right) \lesssim 0.13$ expected from various QCD analyses [30].

It is instructive to elucidate the properties of the fit to the $Z$ parameters in three steps. First, the relatively small sensitivity of the parameter $\bar{s}^{2}\left(m_{Z}^{2}\right)$ to $\alpha_{s}$ can be understood easily, since it is derived essentially from the asymmetry parameters being either completely or nearly insensitive to QCD corrections. Indeed, the fitted values of $\bar{s}^{2}\left(m_{Z}^{2}\right)$ as determined from each asymmetry measurement (see also Fig. 7) turn out to be :

$$
\begin{array}{lll}
\bar{s}^{2}\left(m_{Z}^{2}\right)=0.2309 & \pm 0.0010 & \left(\text { from } A_{\mathrm{FB}}^{0, \ell}\right), \\
\bar{s}^{2}\left(m_{Z}^{2}\right)=0.2316 & \pm 0.0018 & \left(\text { from } P_{\tau}\right), \\
\bar{s}^{2}\left(m_{Z}^{2}\right)=0.2365 & \pm 0.0055 & \left(\text { from } A_{\mathrm{LR}}^{0}\right), \\
\bar{s}^{2}\left(m_{Z}^{2}\right)=0.2313+0.00004 \frac{\alpha_{s}-0.12}{0.01} \pm 0.0011 & \left(\text { from } A_{\mathrm{FB}}^{0, b}\right), \\
\bar{s}^{2}\left(m_{Z}^{2}\right)=0.2302+0.00004 \frac{\alpha_{s}-0.12}{0.01} \pm 0.0035 & \left(\text { from } A_{\mathrm{FB}}^{0, c}\right),
\end{array}
$$

almost independent of $\bar{g}_{Z}^{2}\left(m_{Z}^{2}\right)$ and $\bar{\delta}_{b}\left(m_{Z}^{2}\right)$. Note that although the quark $(q=b, c)$ forward-backward asymmetries have mild $\alpha_{s}$-dependences due to the perturbative QCD corrections [76], they still can be neglected compared to the experimental uncertainties. From the above asymmetry data alone one finds

$$
\begin{array}{ll}
\bar{s}^{2}\left(m_{Z}^{2}\right)=0.2312 \quad \pm 0.0009 & \left(\text { from } A_{\mathrm{FB}}^{0, \ell}, P_{\tau}, A_{\mathrm{LR}}^{0}\right) \\
\bar{s}^{2}\left(m_{Z}^{2}\right)=0.2312+0.00002 \frac{\alpha_{s}-0.12}{0.01} \pm 0.0007 & \left(\text { from } A_{\mathrm{FB}}^{0, \ell}, P_{\tau}, A_{\mathrm{LR}}^{0}, A_{\mathrm{FB}}^{0, b}, A_{\mathrm{FB}}^{0, c}\right) .
\end{array}
$$

The precision of the above determination of $\bar{s}^{2}\left(m_{Z}^{2}\right)$ from the asymmetry data alone is almost as good as that of the global fit to all the $Z$ parameters. These asymmetry measurements are particularly important for GUT studies, since the parameter $\bar{s}^{2}\left(m_{Z}^{2}\right)$ is directly related to the unifying coupling $\hat{s}^{2}(\mu) \equiv \sin ^{2} \theta_{W}(\mu) \overline{\mathrm{MS}}$ via eq. (2.12).

Next, the best-fit value $\bar{s}^{2}\left(m_{Z}^{2}\right) \approx 0.2313$ is taken to probe the sensitivity of the remaining four observables to the parameters $\bar{g}_{Z}^{2}\left(m_{Z}^{2}\right)$ and $\bar{\delta}_{b}\left(m_{Z}^{2}\right)$. As explained in section 3.1, three of the remaining four observables, $\Gamma_{Z}, \sigma_{h}^{0}$ and $R_{\ell}$, are sensitive to the $\alpha_{s}$ 
value assumed, but only through the combination $\bar{\delta}_{b}\left(m_{Z}^{2}\right)+0.6 \alpha_{s}$ (3.25), or equivalently $\alpha_{s}+1.6 \bar{\delta}_{b}\left(m_{Z}^{2}\right) . \Gamma_{Z}$ is also sensitive to $\bar{g}_{Z}^{2}\left(m_{Z}^{2}\right)$. Hence, a 2-parameter fit to the above three observables for $\bar{s}^{2}\left(m_{Z}^{2}\right)=0.2313$ leads to :

$$
\left.\begin{array}{ll}
\bar{g}_{Z}^{2}\left(m_{Z}^{2}\right) & =0.5547 \pm 0.0017 \\
\alpha_{s}+1.6 \bar{\delta}_{b}\left(m_{Z}^{2}\right)= & 0.106 \pm 0.007
\end{array}\right\} \rho_{\text {corr }}=-0.46 .
$$

The above result is found to be insensitive to the $\alpha_{s}$ value in the range $0.10<\alpha_{s}<$ 0.14. The above result for $\bar{g}_{Z}^{2}\left(m_{Z}^{2}\right)$ is consistent with the global fit (4.3), as may be verified by evaluating $\bar{g}_{Z}^{2}\left(m_{Z}^{2}\right)$ at the minimum of $\chi^{2}\left(\alpha_{s}=0.1029\right)$. The anti-correlation above reflects the fact that $\Gamma_{Z}$ remains unaltered, while increasing $\bar{g}_{Z}^{2}\left(m_{Z}^{2}\right)$ and decreasing $\alpha_{s}+1.6 \bar{\delta}_{b}\left(m_{Z}^{2}\right)$ simultaneously.

Only one $Z$ observable is now left, namely $R_{b}$. In section $3.1 R_{b}$ was found to be sensitive to the parameter $\bar{\delta}_{b}\left(m_{Z}^{2}\right)$ alone. A 1-parameter fit to $R_{b}$ yields :

$$
\bar{\delta}_{b}\left(m_{Z}^{2}\right)=0.0012 \pm 0.0068,
$$

keeping the other parameters fixed at $\bar{s}^{2}\left(m_{Z}^{2}\right)=0.2313, \bar{g}_{Z}^{2}\left(m_{Z}^{2}\right)=0.5542$ and $\alpha_{s}=0.12$. However, this fit is insensitive to variations around the values of the fixed parameters. Note, the SM predicts a negative value of $\bar{\delta}_{b}\left(m_{Z}^{2}\right)$ for large $m_{t}$ (see Fig. (1). Thus, there is poor agreement with the expected large $m_{t}$ behavior of the $Z b_{L} b_{L}$ vertex correction from the present $R_{b}$ measurement alone. Since the parameter $\alpha_{s}$ enters the fit only in the combination $\alpha_{s}+1.6 \bar{\delta}_{b}\left(m_{Z}^{2}\right)$, the fitted $\bar{\delta}_{b}\left(m_{Z}^{2}\right)$ can be interpreted as a constraint of $\alpha_{s}$. From eqs. (4.6) and (4.7) follows

$$
\alpha_{s}=0.104 \pm 0.013 .
$$

The fit to the $Z$ shape parameters with both $\bar{\delta}_{b}$ and $\alpha_{s}$ left free yields :

$$
\left.\begin{array}{l}
\bar{\delta}_{b}\left(m_{Z}^{2}\right)=0.0014 \pm 0.0070 \\
\alpha_{s}\left(m_{Z}\right)=0.103 \pm 0.013
\end{array}\right\} \rho_{\text {corr }}=-0.85 .
$$

The large errors and the strong anti-correlation among them show that it makes little sense to extract $\alpha_{s}$ model-independently from the electroweak experiments on the $Z$-pole, as also noted in ref. 92. The low best-fit value of $\alpha_{s}$ reflects essentially the actual value of $R_{b}$, which is larger than the SM prediction in the range $150 \mathrm{GeV}<m_{t}<200 \mathrm{GeV}$ (see Fig. 8). It is therefore necessary to assume the SM contributions to $\bar{\delta}_{b}\left(m_{Z}^{2}\right)$, and to a lesser extent those to $\bar{g}_{Z}^{2}\left(m_{Z}^{2}\right)$, in order to extract $\alpha_{s}$ from the electroweak $Z$ parameters. The result of such an analysis is given in section 5.4, where consequences of the minimal SM are studied. 
Finally, we present the result of 1-parameter fits to four observables, $\Gamma_{Z}, \sigma_{h}^{0}, R_{\ell}$ and $R_{b}$, respectively, in terms of the parameter $\bar{\delta}_{b}\left(m_{Z}^{2}\right)$, for various values of $\bar{g}_{Z}^{2}\left(m_{Z}^{2}\right), \bar{s}^{2}\left(m_{Z}^{2}\right)$ and $\alpha_{s}$. Here, we neglect correlations in the errors and find:

$$
\begin{aligned}
& \bar{\delta}_{b}\left(m_{Z}^{2}\right)=-0.0068-0.0084 \frac{\bar{g}_{Z}^{2}\left(m_{Z}^{2}\right)-0.5542}{0.0017}+0.0020 \frac{\bar{s}^{2}\left(m_{Z}^{2}\right)-0.2313}{0.0007}-0.0061 \frac{\alpha_{s}-0.12}{0.01} \pm 0.0077 \\
& \left(\text { from } \Gamma_{Z}\right) \text {, } \\
& \bar{\delta}_{b}\left(m_{Z}^{2}\right)=-0.0210+0.0000 \frac{\bar{g}_{Z}^{2}\left(m_{Z}^{2}\right)-0.5542}{0.0017}+0.0004 \frac{\bar{s}^{2}\left(m_{Z}^{2}\right)-0.2313}{0.0007}-0.0063 \frac{\alpha_{s}-0.12}{0.01} \pm 0.0168 \\
& \text { (from } \sigma_{h}^{0} \text { ), } \\
& \bar{\delta}_{b}\left(m_{Z}^{2}\right)=-0.0078+0.0000 \frac{\bar{g}_{Z}^{2}\left(m_{Z}^{2}\right)-0.5542}{0.0017}+0.0011 \frac{\bar{s}^{2}\left(m_{Z}^{2}\right)-0.2313}{0.0007}-0.0061 \frac{\alpha_{s}-0.12}{0.01} \pm 0.0044 \\
& \text { (from } R_{\ell} \text { ), } \\
& \bar{\delta}_{b}\left(m_{Z}^{2}\right)=0.0012+0.0000 \frac{\bar{g}_{Z}^{2}\left(m_{Z}^{2}\right)-0.5542}{0.0017}-0.0001 \frac{\bar{s}^{2}\left(m_{Z}^{2}\right)-0.2313}{0.0007}-0.0001 \frac{\alpha_{s}-0.12}{0.01} \pm 0.0068 \\
& \text { (from } R_{b} \text { ). }
\end{aligned}
$$

The above fits clearly confirm quantitatively our observations that $\Gamma_{Z}, \sigma_{h}^{0}$ and $R_{\ell}$ measure the combination $\bar{\delta}_{b}+0.6 \alpha_{s}$ (3.25), that $\Gamma_{Z}$ is also sensitive to $\bar{g}_{Z}^{2}\left(m_{Z}^{2}\right)$, and that $R_{b}$ is sensitive only to $\bar{\delta}_{b}\left(m_{Z}^{2}\right)$. At present the data $\Gamma_{Z}, \sigma_{h}^{0}$ and $R_{\ell}$ favor a negative $\bar{\delta}_{b}\left(m_{Z}^{2}\right)$ value consistent with the SM prediction for $150 \mathrm{GeV}<m_{t}<200 \mathrm{GeV}$, while $R_{b}$ data gives a $\bar{\delta}_{b}\left(m_{Z}^{2}\right)$ value consistent with zero, at $\alpha_{s} \approx 0.12$. The combination of all the above measurements together with all the asymmetry data, and properly accounting for the correlations in the errors, yields

$\bar{\delta}_{b}\left(m_{Z}^{2}\right)=-0.0062-0.0014 \frac{\bar{g}_{Z}^{2}\left(m_{Z}^{2}\right)-0.5542}{0.0017}+0.0009 \frac{\bar{s}^{2}\left(m_{Z}^{2}\right)-0.2313}{0.0007}-0.0046 \frac{\alpha_{s}-0.12}{0.01} \pm 0.0031$,

in accordance with the result (4.3). Note that the coefficient in front of $\alpha_{s}$ in eq. (4.11) is smaller than 0.6 in the combination (3.25) as a consequence of including the additional information due to $R_{b}$.

\subsection{Low energy neutral current experiments}

The two universal parameters $\bar{s}^{2}(0)$ and $\bar{g}_{Z}^{2}(0)$ can be extracted from four types of low energy neutral current experiments : the neutrino-nucleon scattering $\left(\nu_{\mu}-q\right)$, the neutrino-electron scattering $\left(\nu_{\mu}-e\right)$, atomic parity violation (APV) and the polarized electron-deuteron scattering experiments $(e-\mathrm{D})$. Effects due to small, but finite, momentum transfer in these processes are accounted for by assuming the running of these form factors to be governed by the SM particles only (see Fig. 2), which, at low energies, is an excellent approximation. Vertex and box corrections are calculated by assuming that they are dominated by the SM contributions. For details of the theoretical predictions, 
see section 3.2. For each sector, first a model-independent parametrization of the data is given, and then the fit result in the $\left(\bar{s}^{2}(0), \bar{g}_{Z}^{2}(0)\right)$ plane.

\subsubsection{Neutral currents in $\nu_{\mu}-q$ scattering}

For the $\nu_{\mu}-q$ data, the results of the analysis of ref. [78 are adopted. In terms of the model-independent parameters $\left(g_{L}^{2}, g_{R}^{2}, \delta_{L}^{2}, \delta_{R}^{2}\right)$, the following fit has been obtained:

$$
\begin{aligned}
& g_{L}^{2}=\left[0.2982-0.0058\left(m_{c}-1.5\right)\right] \pm 0.0028 \pm 0.0029 \\
& g_{R}^{2}=\left[0.0309-0.0053\left(m_{c}-1.5\right)\right] \pm 0.0034 \pm 0.0028 \\
& \delta_{L}^{2}=\left[-0.0588-0.0025\left(m_{c}-1.5\right)\right] \pm 0.0233 \pm 0.0042 \\
& \delta_{R}^{2}=\left[0.0206+0.0010\left(m_{c}-1.5\right)\right] \pm 0.0155 \pm 0.0039
\end{aligned}
$$

where the former and the latter errors denote the experimental and the parametrization errors. The correlation matrices for the two types of uncertainties also quoted in ref. [78] are respectively

$$
\rho_{\text {corr }}^{(\exp )}=\left(\begin{array}{rrrr}
1 & -0.751 & -0.100 & 0.118 \\
& 1 & 0.064 & 0.097 \\
& & 1 & -0.436 \\
& & & 1
\end{array}\right), \quad \rho_{\text {corr }}^{(\mathrm{par})}=\left(\begin{array}{rrrr}
1 & -0.914 & -0.975 & 0.606 \\
& 1 & 0.945 & -0.677 \\
& & 1 & -0.712 \\
& & & 1
\end{array}\right) .
$$

The fitted parameters depend on the assumed value of the charm quark mass $\left(m_{c}\right.$ in $\mathrm{GeV}$ units) 93] entering the slow-rescaling formula [94] for the charged current cross sections. The data [78] constrain the charm quark mass to

$$
m_{c}=1.54 \pm 0.33 \mathrm{GeV}
$$

After summing the experimental and the parametrization errors in quadrature, and integrating out the $m_{c}$ dependence of the above parametrization under the constraint (4.14), the new model-independent parametrization of the $\nu_{\mu}-q$ data gives :

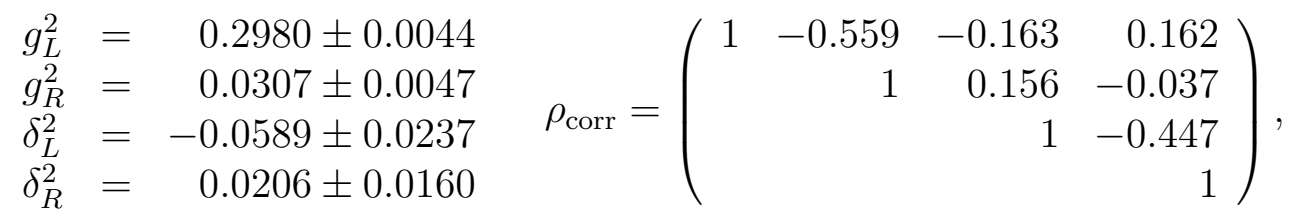

which properly accounts for the uncertainty in $m_{c}$. The parametrization (4.15) serves as input to our analysis.

By using the theoretical formulae (3.44) and (3.45) of section 3.2.1 the data (4.15) can be confronted with the predictions in terms of $\bar{s}^{2}(0)$ and $\bar{g}_{Z}^{2}(0)$. Corrections due to small, but finite, momentum transfer are evaluated at

$$
\langle-t\rangle_{n . c .}=\langle-t\rangle_{\text {c.c. }}=20 \mathrm{GeV}^{2}
$$


in eqs. (3.47) and (3.51) and in the running of $\bar{s}^{2}(t)$ : see eq. (3.56). The fit result is :

$$
\left.\begin{array}{c}
\bar{g}_{Z}^{2}(0)=0.5500_{-0.0083}^{+0.0077} \\
\bar{s}^{2}(0)=0.2420_{-0.0142}^{+0.0130}
\end{array}\right\} \quad \rho_{\text {corr }}=0.916,
$$

Asymmetric errors are quoted. The non-gaussian behaviour of the $\chi^{2}$ function reflects the non-linear transformation between the charge form factors $\left(\bar{g}_{Z}^{2}(0), \bar{s}^{2}(0)\right)$ and the modelindependent parameters $\left(g_{L}^{2}, g_{R}^{2}, \delta_{L}^{2}, \delta_{R}^{2}\right)$, as seen in Fig. 10. The strong positive correlation between the fitted values of $\bar{s}^{2}(0)$ and $\bar{g}_{Z}^{2}(0)$ is a consequence of eq. (4.15): the precisely measured combination $g_{L}^{2}+g_{R}^{2}$ in (4.15) dominates the total neutral current cross section off isoscalar targets. The 1- $\sigma$ contour of the above fit is shown in Fig. 16. It can be reproduced rather accurately by the following parametrization:

$$
\begin{aligned}
& \left.\begin{array}{l}
\bar{g}_{Z}^{2}(0)=0.5497 \pm 0.0080 \\
\bar{s}^{2}(0)=0.2413 \pm 0.0136
\end{array}\right\} \quad \rho_{\text {corr }}=0.916, \\
& \chi_{\min }^{2}=0.13 \text {, }
\end{aligned}
$$

which serves merely for estimating the constraints from the $\nu_{\mu}-q$ experiments. We stress that all the quantitative analyses in the following sections are performed by fitting directly to the original parametrization of the data (4.15).

\subsubsection{Neutral currents in $\nu_{\mu}-e$ scattering}

The $\nu_{\mu}-e$ data from the three experiments: CHARM, BNL E374 and CHARM-II [84], have been summarized in ref. [79] in terms of the model-independent parameters $s_{\nu e}^{2}$ and $\rho_{\nu e}$ :

$$
\left.\begin{array}{ll}
\rho_{\nu e} \equiv(\rho)_{\text {eff }}^{\nu_{\mu} e} & =1.007 \pm 0.028 \\
s_{\nu e}^{2} \equiv\left(\sin ^{2} \theta_{W}\right)_{\text {eff }}^{\nu_{\mu} e} & =0.233 \pm 0.008
\end{array}\right\} \quad \rho_{\text {corr }}=0.09 .
$$

As explained in detail in section 3.2.2, first the total cross section $\sigma^{\nu e}$ and $\sigma^{\bar{\nu} e}$ is reconstructed by using the formula (3.75), and then the fit is performed by using the theoretical expressions (3.64). The reconstructed cross sections are found to be

$$
\left.\begin{array}{l}
\sigma^{\nu e} / E_{\nu}\left(10^{-42} \mathrm{~cm}^{2} / \mathrm{GeV}\right)=1.56 \pm 0.10 \\
\sigma^{\bar{\nu} e} / E_{\bar{\nu}}\left(10^{-42} \mathrm{~cm}^{2} / \mathrm{GeV}\right)=1.36 \pm 0.09
\end{array}\right\} \quad \rho_{\text {corr }}=0.51
$$

and the 2-parameter fit to the above data gives

$$
\left.\begin{array}{l}
\bar{g}_{Z}^{2}(0)=0.5459 \pm 0.0154 \\
\bar{s}^{2}(0)=0.2416 \pm 0.0079
\end{array}\right\} \quad \rho_{\text {corr }}=0.09
$$

The same result follows if we use the approximation (3.74) directly to fit the parametrization (4.19). Here $\chi_{\min }^{2}=0$, since the number of degrees is $2-2=0$. The result is 
shown in Fig. 16 by the 1- $\sigma$ contour. The weak mixing form factor $\bar{s}^{2}(0)$ is measured more accurately in the $\nu_{\mu}-e$ experiments than in the $\nu_{\mu}-q$ experiments, whereas for $\bar{g}_{Z}^{2}(0)$ it is the other way around.

\subsubsection{Atomic parity violation}

As for the APV experiments the result of the analysis 80 on the parity violating transitions in the cesium atom $(A, Z)=(133,55)$ are used :

$$
Q_{W}(135,55)=-71.04 \pm 1.81 \text {. }
$$

The quoted uncertainty is the quadratic sum of experimental and theoretical errors. After correcting for the vertex and box corrections [85] as explained in detail in section 3.2.3, one finds

$$
\bar{s}^{2}(0)=0.2294-0.6178\left[\bar{g}_{Z}^{2}(0)-0.5486\right] \pm 0.0082 .
$$

Here the value $\bar{g}_{Z}^{2}(0)=0.5486$ stands for the prediction at $\bar{\rho}=1$ or $T=\bar{\delta}_{G} / \alpha$. The result is shown in Fig. 16 by 1- $\sigma$ contours. As anticipated in the previous section, the correlation between the fitted $\bar{s}^{2}(0)$ and $\bar{g}_{Z}^{2}(0)$ values is opposite to that from $\nu-q$ fit. As a consequence, the constraints on both $\bar{s}^{2}(0)$ and $\bar{g}_{Z}^{2}(0)$ are improved significantly by combining the two types of experiments.

\subsubsection{Polarization asymmetry in $e-\mathrm{D}$ scattering}

Finally, for the SLAC $e \mathrm{D}$ polarization asymmetry experiment [87 a model-independent fit is performed to the original data by using the two combinations, $2 C_{1 u}-C_{1 d}$ and $2 C_{2 u}-C_{2 d}$ of the coefficients of the effective parity violating $e-q$ neutral current operators [24]: see eq. (3.76). In the quark parton model with the valence quark approximation the observed polarization asymmetry is expressed in terms of the above parameters by

$$
-\frac{A}{Q^{2}}=\frac{6 G_{F}}{5 \sqrt{2} \bar{e}^{2}\left(-Q^{2}\right)}\left\{\left(2 C_{1 u}-C_{1 d}\right)+\left(2 C_{2 u}-C_{2 d}\right) \frac{1-(1-y)^{2}}{1+(1-y)^{2}}\right\},
$$

which depends on the scaling variable $y$, but not on $x$. The mild $Q^{2}$ dependence due to the running of the effective QED charge $\bar{e}^{2}\left(-Q^{2}\right)$ is accounted for. There have been extensive studies [95,96], which show that the above approximation is in fact valid on more general grounds, but that it may suffer from higher-twist contributions. We therefore perform a new model-independent fit to the original data [87], and obtain quantitatively the theoretical uncertainty in the fitted parameters.

By taking account of the sea-quark contributions and finite $R=\sigma_{L} / \sigma_{T}$ [95], as well as possible higher twist contributions [96,97], the above simple expression for the asymmetry 
(4.24) is modified as follows :

$$
-\frac{A}{Q^{2}}=\frac{6 G_{F}}{5 \sqrt{2} \bar{e}^{2}\left(-Q^{2}\right)}\left\{\left(2 C_{1 u}-C_{1 d}\right)\left(1-\frac{3}{4} c\right)+\left(2 C_{2 u}-C_{2 d}\right)\left(b+\frac{5}{12} c\right)\right\},
$$

with

$$
\begin{aligned}
& b=\frac{1-(1-y)^{2}}{1+(1-y)^{2}-y^{2} \frac{R}{1+R}}\left(1+8.35 \delta-\epsilon_{u}-\frac{\epsilon_{s}}{5}\right), \\
& c=1.34 \delta-\epsilon_{s} / 5 .
\end{aligned}
$$

Here the $x$-dependent parameters $\epsilon_{u}$ and $\epsilon_{s}$ denote the relative contribution of the sea $u$-quark and that of $s$ and $\bar{s}$ quarks, respectively, which are parametrized by

$$
\begin{aligned}
\epsilon_{u} & =\epsilon \frac{(1-x)^{4}}{\sqrt{x}}, \\
\epsilon_{s} & =\frac{\epsilon}{3} \frac{(1-x)^{4}}{\sqrt{x}} .
\end{aligned}
$$

The uncertainty in the factor $\epsilon$ above is estimated to be

$$
\epsilon=0.1 \pm 0.03
$$

The effects of introducing sea-quark contributions in the fit is shown in Fig. 17(a). As found in ref. [95], the effect is very small along the tree level SM prediction as shown by the straight line in the figure. Some representative values of $\sin ^{2} \theta_{W}$ in the SM are denoted by blobs. The longitudinal to transverse cross section ratio $R=\sigma_{L} / \sigma_{T}$ is allowed to vary within the rather conservative limits

$$
R=0.2 \pm 0.2
$$

The effect of introducing the $R$ parameter alone is shown in Fig. 17(b) and the result turns out to be insensitive to its uncertainty, especially along the tree-level SM trajectory, confirming the earlier observation of ref. [95]. Finally, the parameter $\delta$ in the factors $b$ and $c$ parametrizes the higher twist effects as expected in the MIT bag model [97]. Taking as the magnitude of the uncertainty the largest value of the MIT bag model estimate of ref. 97 yields

$$
\delta=(1.58 \pm 1.58) \times 10^{-3}
$$

The effects of introducing the $\delta$ parameter alone are shown in Fig. 17(c). As in the case of the sea-quark contributions (Fig. 17(a)), the effect is negligibly small along the line representing the tree-level SM prediction. Note that the higher-twist effects are found to 
be rather model dependent [98]. The MIT-Bag model estimates [97] adopted here lead to quite small corrections, as in the neutrino scattering off isoscalar targets [99]. Further study on the higher twist effects may be needed to achieve precision measurements of the electroweak parameters in these reactions.

After allowing for all of the above uncertainties, one finds

$$
\left.\begin{array}{l}
2 C_{1 u}-C_{1 d}=+0.94 \pm 0.26 \\
2 C_{2 u}-C_{2 d}=-0.66 \pm 1.23
\end{array}\right\} \quad \rho_{\text {corr }}=-0.975
$$

with $\chi_{\min }^{2}=9.95$ for 11 data points, that is, a good fit. The above result is shown in Fig. 17(d). Because of the strong correlation, only a linear combination of the two coupling factors is measured well.

By using the theoretical formulae (3.78), a fit is made to the data (4.33) in terms of the two parameters $\bar{s}^{2}(0)$ and $\bar{g}_{Z}^{2}(0)$. In order to fix the $q^{2}$-dependent factors $\left(Q^{2} \equiv-q^{2}\right)$ $\Gamma_{1}\left(-Q^{2}\right), \bar{\Gamma}_{2}\left(-Q^{2}\right)$ and $\bar{s}^{2}\left(-Q^{2}\right)$ in the amplitudes we choose $\left\langle Q^{2}\right\rangle=1.5 \mathrm{GeV}^{2}$. Note, however, that $Q^{2}$-dependence of each data point [87] and that of the QED running coupling $\bar{e}^{2}\left(-Q^{2}\right)$ in eq. (4.25) have been respected in the model-independent fit (4.33). The result is :

$$
\begin{aligned}
\bar{s}^{2}(0) & =0.2273+0.3067\left[\bar{g}_{Z}^{2}(0)-0.5486\right] \pm 0.0092, \\
\chi_{\min }^{2} & =0.46-1.77\left[\bar{g}_{Z}^{2}(0)-0.5486\right],
\end{aligned}
$$

and shown in Fig. 16. Note that the parametrization (4.34b) is valid only in the vicinity of the SM predictions $\bar{g}_{Z}^{2}(0) \sim 0.55$ (but is valid in the whole region of Fig. 16), and that the global $\chi_{\min }^{2}$ is zero, since the two parameter parametrization (4.33) is adopted as the original data of our fit.

\subsubsection{Summary of low energy neutral current experiments}

In this section the fits to the electroweak observables in the four low energy neutral current experiments are summarized. The fit results are illustrated in Fig. 16 by 1$\sigma$ allowed regions in the $\left(\bar{s}^{2}(0), \bar{g}_{Z}^{2}(0)\right)$ plane. Since all four pieces of information are consistent with each other, a combined fit can been performed :

$$
\left.\begin{array}{c}
\bar{g}_{Z}^{2}(0)=0.5462 \pm 0.0036 \\
\bar{s}^{2}(0)=0.2353 \pm 0.0044
\end{array}\right\} \quad \rho_{\text {corr }}=0.53,
$$

The fit with $7=9-2$ degrees of freedom is good and its result is shown in Fig. 16 by the ellipse with the thick $1-\sigma$ contour. 
It is sometimes useful to analyse the neutral current sector with and without inclusion of the neutrino data, since in some models they receive different new physics contributions. To this end the fit is done separately for $\nu_{\mu}-q$ and $\nu_{\mu}-e$ experiments :

$$
\left.\begin{array}{c}
\bar{g}_{Z}^{2}(0)=0.5496 \pm 0.0068 \\
\bar{s}^{2}(0)=0.2414 \pm 0.0047
\end{array}\right\} \quad \rho_{\text {corr }}=0.75,
$$

The fit for the APV and $e \mathrm{D}$ experiments gives :

$$
\left.\begin{array}{c}
\bar{g}_{Z}^{2}(0)=0.5510 \pm 0.0165 \\
\bar{s}^{2}(0)=0.2280 \pm 0.0088
\end{array}\right\} \quad \rho_{\text {corr }}=-0.62,
$$

These two fits are again consistent and their combination reproduces, of course, the above global fit (4.35).

\subsection{Charged current experiments}

The $W$ mass measurements have been updated recently by the CDF and D0 collaborations. By combining the most recent measurements 88 and the previous result of PDG [25] one obtains

$$
m_{W}=80.24 \pm 0.16 \mathrm{GeV} .
$$

Note that in this analysis the $W$ mass definition follows the LEP convention [3], as opposed to the pole mass definition: see eq. (2.14). The pole mass should be smaller by about $0.03 \mathrm{GeV}$. The difference is still negligibly small as compared to the error of $0.16 \mathrm{GeV}$. It is worth noting that the $W$ propagator with running width factor gives a more accurate description of the scattering amplitudes when no imaginary parts are introduced outside the propagator factor.

The electroweak parameter $\bar{g}_{W}^{2}(0)$ is now obtained by combining the $m_{W}$ measurement with the $\mu$ life-time parameter $G_{F}$ (3.99) : we find

$$
\bar{g}_{W}^{2}(0)=0.4225-0.0031 \frac{\bar{\delta}_{G}-0.0055}{\alpha} \pm 0.0017,
$$

where $\bar{\delta}_{G}=0.0055$ is the SM estimate for the process specific correction to the $\mu$ life-time: see eq. (3.98). No other experiment in the charged current sector is accurate enough to provide adequate information for our electroweak analysis. Precise measurements of the $W$ shape parameters 100 would improve our knowledge in this sector considerably. 


\section{Systematic analysis}

In this section, first the $q^{2}$-dependence of the two charge form factors $\bar{g}_{Z}^{2}\left(q^{2}\right)$ and $\bar{s}^{2}\left(q^{2}\right)$ is examined between $q^{2}=0$ and $q^{2}=m_{Z}^{2}$. Next a combined fit in terms of the $S, T$ and $U$ parameters is made assuming the $q^{2}$-dependence of these charge form factors to be governed by the SM. Finally, only the SM particles are assumed to contribute to the radiative effects and the preferred range of the two mass parameters $m_{t}$ and $m_{H}$ is searched for. Also the $\alpha_{s}$ and $\delta_{\alpha}$ dependences of the fits are discussed in detail.

\subsection{Summary of all experimental constraints on the electroweak parameters}

The information on all electroweak precision data has been represented in the previous sections in terms of the charge form factor values (see eqs. (4.3), (4.35) and (4.39)) and is, for convenience, collected in Table 6. In addition, the fine structure constant $\alpha$ determining the charge form factor $\bar{e}^{2}(0)=4 \pi \alpha$ (see Tables 1 and 2) has been used as an input parameter. In calculating $\chi^{2}$ the model-independent parametrizations of the original data are used as inputs for the fit : eqs. (4.1)-(4.2) for the $Z$ parameters (section 4.1), eq. (4.15) for the $\nu_{\mu}-q$ scattering experiments (section 4.2.1), eq. (4.19) for the $\nu_{\mu}-e$ scattering experiments (section 4.2.2), eq. (4.22) for the atomic parity violation experiments (section 4.2.3), eq. (4.33) for the $e-\mathrm{D}$ polarization asymmetry measurements (section 4.2.3), and eq. (4.38) for the $W$ mass measurements (section 4.3). The $\chi^{2}$ fits in each of the various sectors look all fine and it is concluded that the whole body of data is consistent with the assumption of the $\mathrm{SU}(2)_{\mathrm{L}} \times \mathrm{U}(1)_{\mathrm{Y}}$ universality and the SM dominance of the vertex and box corrections.

\subsection{Testing the running of the charge form factors}

If there are new particles coupled to the weak gauge bosons with masses near or below $m_{W}$ and $m_{Z}$, their signal can be identified as an anomalous running of the charge form factors [11, 12]. In principle, the running of all four charge form factors provides us with information on new physics contributions via eq. (2.30) for $1 / \bar{\alpha}\left(q^{2}\right)$, eq. (2.40a) for $\bar{s}^{2}\left(q^{2}\right) / \bar{\alpha}\left(q^{2}\right)$, eq. (2.40b) for $1 / \bar{g}_{Z}^{2}\left(q^{2}\right)$ and via eq. (2.40G) for $1 / \bar{g}_{W}^{2}\left(q^{2}\right)$. At present, only two of the four form factors, $\bar{s}^{2}\left(q^{2}\right)$ and $\bar{g}_{Z}^{2}\left(q^{2}\right)$, have been determined with sufficient accuracy at two different energy scales, $q^{2}=0$ and $m_{Z}^{2}$.

The results collected in Table 6 yield :

$$
\left.\begin{array}{c}
\frac{4 \pi}{\bar{g}_{Z}^{2}\left(m_{Z}^{2}\right)}-\frac{4 \pi}{\bar{g}_{Z}^{2}(0)}=-0.33+1.2\left(\alpha_{s}-0.12\right) \pm 0.17 \\
\frac{\bar{s}^{2}\left(m_{Z}^{2}\right)}{\bar{\alpha}\left(m_{Z}^{2}\right)_{\mathrm{SM}}}-\frac{\bar{s}^{2}(0)}{\alpha}=-2.47+1.1\left(\alpha_{s}-0.12\right) \pm 0.62
\end{array}\right\} \quad \rho_{\text {corr }}=-0.49 .
$$


In the absence of a precise value for $\bar{\alpha}\left(m_{Z}^{2}\right)$ the SM prediction $\bar{\alpha}\left(m_{Z}^{2}\right)_{\mathrm{SM}}=1 / 128.72$ (or, more generally, $\left.\delta_{\alpha} \equiv 1 / \bar{\alpha}\left(m_{Z}^{2}\right)-128.72=0\right)$ is used above.

Fig. 18 illustrates SM running of the charge form factor $\bar{g}_{Z}^{2}\left(q^{2}\right)$,

$$
\frac{4 \pi}{\bar{g}_{Z}^{2}\left(m_{Z}^{2}\right)}-\frac{4 \pi}{\bar{g}_{Z}^{2}(0)}=\frac{1}{4}\left[S_{Z}\left(m_{Z}^{2}\right)-S_{Z}(0)\right]
$$

as a function of $m_{H}$, together with the experimental constraint (5.1). The $q^{2}$-dependent $S_{Z}$ function is defined in terms of the gauge boson two-point functions in eq. (B.41a) of appendix B. The difference (5.2) takes the form (2.40b) of section 2. The $m_{t}$ dependence of the SM prediction is very small compared to the experimental error for $m_{t}>100 \mathrm{GeV}$. The SM is consistent with the data as long as the Higgs boson mass is not too small. Note that the $1-\sigma$ constraint on $m_{H}, m_{H}>2.9 \mathrm{GeV}(67 \% \mathrm{CL})$, is obtained merely by comparing the $Z$ boson coupling strengths at $q^{2}=0$ and $q^{2}=m_{Z}^{2}$. These values are, however, obtained by neglecting the $Z \rightarrow H f \bar{f}$ contribution to $\Gamma_{Z}$, and are anyway excluded by direct searches at LEP $\left(m_{H}>63 \mathrm{GeV}\right)$ 101].

The Higgs boson does not contribute to the running of the other neutral current form factors, $1 / \bar{\alpha}\left(q^{2}\right)$ and $\bar{s}^{2}\left(q^{2}\right) / \bar{\alpha}\left(q^{2}\right)$. They are affected by loops of charged particles only, and, for instance, the top quark contributions to the running of these form factors are parametrized in appendix B, in eqs. (B.27) and (B.28):

$$
\begin{aligned}
\frac{1}{\bar{\alpha}\left(m_{Z}^{2}\right)_{\mathrm{SM}}} & =128.71+\delta_{\mathrm{had}}+0.024\left(1+5 \frac{\alpha_{s}}{\pi}\right)\left(\frac{100 \mathrm{GeV}}{m_{t}}\right)^{2}, \\
{\left[\frac{\bar{s}^{2}\left(m_{Z}^{2}\right)}{\bar{\alpha}\left(m_{Z}^{2}\right)}-\frac{\bar{s}^{2}(0)}{\alpha}\right]_{\mathrm{SM}} } & =-3.09+\frac{\delta_{\mathrm{had}}}{2}+0.009\left(1+5 \frac{\alpha_{s}}{\pi}\right)\left(\frac{100 \mathrm{GeV}}{m_{t}}\right)^{2},
\end{aligned}
$$

The $m_{t}$ dependences of these runnings are very small for $m_{t} \gtrsim 100 \mathrm{GeV}$.

The running may be appreciable, if there is a charged fermion with mass near to half the $Z$ mass [12]. The case of a light wino, the fermionic partner of the $W$ in the supersymmetric SM, is shown in Fig. 19: (a) $4 \pi / \bar{g}_{Z}^{2}\left(m_{Z}^{2}\right)-4 \pi / \bar{g}_{Z}^{2}(0)$, (b) $\bar{s}^{2}\left(m_{Z}^{2}\right) / \bar{\alpha}\left(m_{Z}^{2}\right)-$ $\bar{s}^{2}(0) / \alpha$, and (c) $\delta_{\alpha} \equiv 1 / \bar{\alpha}\left(m_{Z}^{2}\right)-128.72$. The singularity at $m_{\text {wino }}=m_{Z} / 2$ of the charge form factor $4 \pi / \bar{g}_{Z}^{2}\left(m_{Z}^{2}\right)$ in (a) reflects [65] the deviation of the $Z$ line-shape from the Breit-Wigner form assumed both in the experimental fit and the corresponding theoretical formulae, and is unphysical. The $1-\sigma$ bound on the wino mass, $m_{\text {wino }}>46.1 \mathrm{GeV}$, as read off from Fig. 19 is unrealistic, since the threshold $2 m_{\text {wino }}=92.2 \mathrm{GeV}$ is less than a half width away from the $Z$-pole. In order to derive constraints on particles very near to the threshold, one should look for deviations of the $Z$ line shape from the simple BreitWigner form [37, 65]. When calculating the predictions for (b) and (c) the hadronic vacuum polarization contribution to the running of these form factors is set to $\delta_{\text {had }}=0$, while the present estimate [28] is $\delta_{\text {had }}=0 \pm 0.1$ (B.22). Wino of masses around $50 \mathrm{GeV}$ 
may shift $\delta_{\alpha}=1 / \bar{\alpha}\left(m_{Z}^{2}\right)-128.72$ from its canonical value $\delta_{\alpha}=0$ by about 0.1 , which is of the same order as the present uncertainty in the SM prediction.

It is clearly seen from Fig. 18 and from eq. (5.3) that the results (5.1) are consistent with the SM predictions in the range $m_{H}>60 \mathrm{GeV}$ and $m_{t}>100 \mathrm{GeV}$. The study of the two examples, a very light Higgs boson and a supersymmetric wino, demonstrates that more accurate values of $\bar{s}^{2}(0)$ and $\bar{g}_{Z}^{2}(0)$ are needed to detect effects of new physics through the running of the charge form factors. Accurate measurements of the charge form factor $1 / \bar{\alpha}\left(q^{2}\right)$ at $\left|q^{2}\right| \sim m_{Z}^{2}$ should also provide independent information.

Fig. 20 shows the above results in the $\left(\bar{s}^{2}\left(m_{Z}^{2}\right), \bar{g}_{Z}^{2}\left(m_{Z}^{2}\right)\right)$ plane, where the $Z$ parameter fit ('LEP+SLC') is taken from Fig. 15 for $\alpha_{s}=0.12$, and the combined low energy fit of Fig. 16 has been rescaled to the $m_{Z}$ scale by assuming SM running of the two charge form factors, $\bar{s}^{2}\left(q^{2}\right)$ and $\bar{g}_{Z}^{2}\left(q^{2}\right)$. The combined low energy neutral current data (see Table [6 and Fig. [16) are displayed for various choices of $m_{t}$ and $m_{H}$ in order to put in evidence their small, but finite, effects on the running of these form factors. The four contours are obtained for $m_{t}=100,200 \mathrm{GeV}$ and $m_{H}=60,1000 \mathrm{GeV}$. At $m_{t}=175 \mathrm{GeV}$ and $m_{H}=100 \mathrm{GeV}$, the fit (4.35a) for the low energy neutral current data can be reparametrized as

$$
\left.\begin{array}{l}
\bar{g}_{Z}^{2}\left(m_{Z}^{2}\right)=0.5533 \pm 0.0037 \\
\bar{s}^{2}\left(m_{Z}^{2}\right)=0.2266 \pm 0.0047
\end{array}\right\} \quad \rho_{\text {corr }}=0.53 .
$$

It is seen from the figure that the low energy neutral current fit and the $Z$ parameter fit in terms of the charge form factors $\bar{s}^{2}\left(q^{2}\right)$ and $\bar{g}_{Z}^{2}\left(q^{2}\right)$ are in accordance with the running of these form factors as predicted by the SM.

The thick solid contour marks the result of the fit to all neutral current experiments as summarized in Table 6 assuming the SM for the running of $\bar{g}_{Z}^{2}\left(q^{2}\right)$ and $\bar{s}^{2}\left(q^{2}\right)$ :

$$
\begin{aligned}
\bar{g}_{Z}^{2}\left(m_{Z}^{2}\right)= & 0.5544-0.00023 \frac{\alpha_{s}-0.12}{\bar{S}_{0} 01} \pm 0.0015 \\
\bar{s}^{2}\left(m_{Z}^{2}\right)= & 0.2312+0.00008 \frac{\alpha_{s}-0.12}{0.01} \pm 0.0007 \\
\bar{\delta}_{b}\left(m_{Z}^{2}\right)= & -0.0064-0.00437 \frac{\alpha_{s}-0.12}{0.01} \pm 0.0034 \\
& \chi_{\min }^{2}=4.67+\left(\frac{\alpha_{s}-0.1024}{0.0127}\right)^{2} .
\end{aligned}
$$

In the global fit the uncertainty due to $m_{t}$ and $m_{H}$ in the running of the form factors is negligible in the range $m_{t}=100-200 \mathrm{GeV}$ and $m_{H}=60-1000 \mathrm{GeV}$. The $\chi_{\min }^{2}$ value of 6.6 for $\alpha_{s}=0.12$ is acceptable for $15(=18-3)$ degrees of freedom. In conclusion, there is no indication of new particles with mass $\lesssim m_{Z}$ in the running of the charge form factors.

Note that the errors in (5.1) are dominated by those of the low energy experiments. Further improvements in the low energy precision experiments are required to detect a signal of relatively light new particles, should they exist, through anomalous running of 
the charge form factors. In comparing the global fit of Fig. 20 with the individual fit to the low energy $N C$ data in Fig. 16, the fit from the $\nu_{\mu}-q$ and $\nu_{\mu}-e$ experiments (4.36) are remarkably consistent with the $Z$ parameter fit of Table 6, whereas the fit of the $e-q$ sector (4.37) based on the APV and the $e-\mathrm{D}$ asymmetry measurements are about 1.5 standard deviations away. For $m_{t}=175 \mathrm{GeV}$ and $m_{H}=100 \mathrm{GeV}$, the fit (4.36a) for $\nu_{\mu}-q$ and $\nu_{\mu}-e$ scattering is re-parametrized as

$$
\left.\begin{array}{l}
\bar{g}_{Z}^{2}\left(m_{Z}^{2}\right)=0.5568 \pm 0.0048 \\
\bar{s}^{2}\left(m_{Z}^{2}\right)=0.2331 \pm 0.0072
\end{array}\right\} \quad \rho_{\text {corr }}=0.75,
$$

while the fit (4.37a) for the APV and polarized $e-\mathrm{D}$ experiments gives

$$
\left.\begin{array}{l}
\bar{g}_{Z}^{2}\left(m_{Z}^{2}\right)=0.5583 \pm 0.0170 \\
\bar{s}^{2}\left(m_{Z}^{2}\right)=0.2188 \pm 0.0093
\end{array}\right\} \quad \rho_{\text {corr }}=-0.62 .
$$

Further studies of polarization asymmetries in the $e-q$ sector as well as studies of the neutral current processes at TRISTAN energies might be potentially rewarding.

\subsection{Testing the 3 parameter universality}

Once the $q^{2}$-dependence of the charge form factors is assumed to be governed by SM physics alone, all radiative effects to the gauge bosons depend on three universal parameters : $S, T, U$. They include the SM radiative effects as well as new physics contributions, as opposed to the original definitions of ref. [4]. While the charge form factors $\bar{g}_{Z}^{2}\left(m_{Z}^{2}\right), \bar{s}^{2}\left(m_{Z}^{2}\right), \bar{g}_{W}^{2}(0)$ can be directly confronted with experiments, the $S, T, U$ parameter fit suffers from uncertainty in the QED effective coupling $\bar{\alpha}\left(m_{Z}^{2}\right)$, the reason being the fact that the charge form factors are determined by the $S, T, U$ parameters under the $\left(\alpha, G_{F}, m_{Z}\right)$ constraints (see discussion in section 2.3). The magnitude of $\bar{\alpha}\left(m_{Z}^{2}\right)$ is controlled by the external parameter $\delta_{\alpha} \equiv 1 / \bar{\alpha}\left(m_{Z}^{2}\right)-128.72$.

A 4-parameter fit yields :

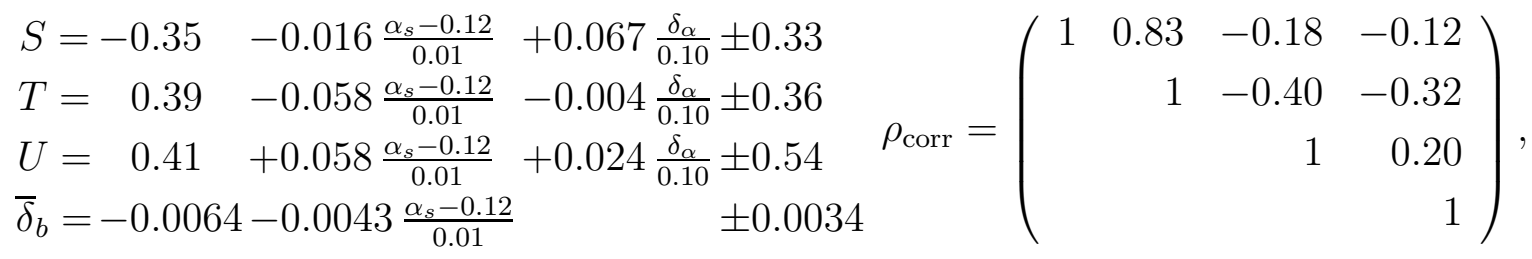

from the result of the global fit summarized in Table 6. The best-fit values of $S, T, U$ and $\bar{\delta}_{b}$ are weakly dependent upon $\alpha_{s}$ and $\delta_{\alpha}$ as quoted explicitly in eq. (5.8a). The minimum of $\chi^{2}$ turns out to be practically independent of $\delta_{\alpha}$. We therefore add to the fit 
the independent knowledge $\delta_{\alpha}=0.0 \pm 0.1$ [28] leading then to :

$$
\chi_{\min }^{2}=4.67+\left(\frac{\alpha_{s}-0.1024}{0.0127}\right)^{2}+\left(\frac{\delta_{\alpha}}{0.10}\right)^{2} .
$$

The correlation between $S$ and $T$ is strong, since they are constrained by the precisely measured weak mixing form factor $\bar{s}^{2}\left(m_{Z}^{2}\right)$ via eq. (2.38b).

The above results are shown in Fig. 21 by $1-\sigma$ contours as projections onto the $(S, T)$, $(S, U)$, and $(U, T)$ planes. The contours are drawn for three $\alpha_{s}$ values, $\alpha_{s}=0.11$ (dashed lines), 0.12 (solid lines) and 0.13 (dash-dotted lines), and for $m_{t}=150,200 \mathrm{GeV}$ and $m_{H}=100,1000 \mathrm{GeV}$ in the running of the charge form factors $\bar{s}^{2}\left(q^{2}\right)$ and $\bar{g}_{Z}^{2}\left(q^{2}\right)$ between $q^{2}=0$ and $q^{2}=m_{Z}^{2}$. The fit results depend slightly on $m_{t}$ and $m_{H}$ in the above range. The numerical values of eq. (5.8) are obtained for $m_{t}=175 \mathrm{GeV}$ and $m_{H}=100 \mathrm{GeV}$. The SM predictions of appendix $\mathrm{C}$ are drawn in Fig. 21 by lattices in the region $m_{t}=100-200 \mathrm{GeV}$ and $m_{H}=50-1000 \mathrm{GeV}$.

The fitted $T$ parameter depends only slightly on $\alpha_{s}$, when the parameter $\bar{\delta}_{b}$ is allowed to vary freely within the experimental constraints. If we fix $\bar{\delta}_{b}$ by a theoretical model, then the $T$ parameter should have stronger $\alpha_{s}$ dependence due to the correlation -0.31 between the errors of $T$ and $\bar{\delta}_{b}$ (see section 6.3 for more discussions). The $S$ parameter depends on $\delta_{\alpha}$. The fitted $S$ value is shifted by about 0.07 (that is, $20 \%$ of its present uncertainty of 0.33 ) for $\left[\delta_{\alpha}\right]_{\mathrm{SM}} \approx \delta_{\text {had }}=0 \pm 0.10$.

The parameters $S, T$ and $U$ measure electroweak radiative effects in the gauge boson propagators. The fit (5.8) shows that the data favor negative $S$ and positive $T$ at $\alpha_{s}=$ 0.12 and $\delta_{\alpha}=0$. The point $S=T=U=\bar{\delta}_{b}=0$, which represent no electroweak radiative effects in the gauge boson propagators nor in the $Z b_{L} b_{L}$ vertex, is about 4.5 standard deviations away from the minimal for $\alpha_{s}=0.12$ and $\delta_{\alpha}=0$. However, if in addition the electroweak radiative effects are dropped in the muon decay by setting $\bar{\delta}_{G}=0$ in eq. (2.26), then according to the substitution rule (2.39) the 'no-radiative effects' point becomes $T=0.0055 / \alpha=0.75, S=U=\bar{\delta}_{b}=0$ in the fit (5.8), which is only 2.6 standard deviations away from the minimal. Although this result still assumes the SM radiative corrections for the remaining vertex/box corrections, it is essentially the mechanism that led the authors of ref. 64 to state that there had not yet been an evidence for genuine electroweak radiative effects. Our analysis makes it clear that it is more natural to interpret significant radiative effects in the $T$ parameter which are approximately cancelled by the effect of the radiative effect $\bar{\delta}_{G}$ in the prediction of the electroweak observables.

The resulting $\chi_{\min }^{2}$ of eq. (5.8b) agrees nearly with that of eq. (5.5b). The effective number of degrees of freedom is in both cases 15 , namely $19-4$ respectively $18-3$. The fit 
to the $N C$ data contains actually only three parameters, $S, T$ and $\bar{\delta}_{b}\left(m_{Z}^{2}\right)$, corresponding to the charge form factors $\bar{s}^{2}\left(m_{Z}^{2}\right), \bar{g}_{Z}^{2}\left(m_{Z}^{2}\right)$ and $\bar{\delta}_{b}\left(m_{Z}^{2}\right)$ in the global fit. The present fit depends in addition upon $U$, when the charged current data (and thus the forth form factor, $\left.\bar{g}_{W}^{2}(0)\right)$ are included.

\subsection{Testing the Minimal Standard Model}

In the minimal SM, all the parameters $\bar{g}_{Z}^{2}\left(m_{Z}^{2}\right), \bar{s}^{2}\left(m_{Z}^{2}\right), \bar{g}_{Z}^{2}(0), \bar{s}^{2}(0), \bar{g}_{W}^{2}(0)$ and $\bar{\delta}_{b}\left(m_{Z}^{2}\right)$ depend uniquely upon the two mass parameters $m_{t}$ and $m_{H}$. Consequently, the results of the fits summarized in Table 6 are constraining $m_{t}$ and $m_{H}$. We should repeat here that the SM contributions from the top-bottom doublet to the form factors are calculated by using the simple $O\left(\alpha \alpha_{s}\right)$ two-loop formula [54 56]. Non-perturbative $t \bar{t}$ threshold effects [60] 62] will affect these corrections and the predicted $m_{t}$ value will shift upwards by as much as a few $\mathrm{GeV}$ 62] from the effect in the $T$ parameter. Our approach separates clearly the data analysis in terms of the generic form factors and the analysis of the SM contributions to these form factors. Uncertainties in the latter process can hence be studied separately. In fact if the SM $m_{t}$-dependence of the fit is dictated by the $m_{t}$-dependence of the $T$ parameter alone, then the sole effect of the non-perturbative threshold corrections can be expressed as a rescaling of the $m_{t}$ parameter in the following analysis.

Fig. 22 shows the result of the global SM fit to all electroweak data in the $\left(m_{H}, m_{t}\right)$ plane [102, 103 for three representative $\alpha_{s}$ values. The " $\times$ " indicate the minimum of $\chi^{2}$; 7.4, 6.6, 10.3 for $\alpha_{s}=0.11,0.12,0.13$, respectively, the inner contours correspond to $1-\sigma$, the outer to $\chi^{2}=\chi_{\min }^{2}+4.61$ (that is, $90 \% \mathrm{CL}$ ). Dashed lines show the best $m_{t}$ values for a given $m_{H}$. Note the positive correlation between the preferred values of $m_{t}$ and $m_{H}$, which is found to be independent of the assumed $\alpha_{s}$ value. On the other hand, the preferred range of $m_{H}$ depends rather sensitively on $\alpha_{s}$. For the cases $\alpha_{s}\left(m_{Z}\right)=0.11$ and 0.12 smaller $m_{H}$ values are preferred, whereas for $\alpha_{s}\left(m_{Z}\right)=0.13$ larger $m_{H}$ is slightly favored. If the lower bound for $m_{H}, m_{H}>63 \mathrm{GeV}$ at $95 \% \mathrm{CL}$ measured by the LEP experiments [101], is imposed, $m_{t}$ below $100 \mathrm{GeV}$ is clearly disfavored for all $\alpha_{s}$, in agreement with the directly established lower top mass limit 104, 105.

The $\chi^{2}$ function in the global fit to all electroweak data can be represented in terms of the four parameters $m_{t}, m_{H}, \alpha_{s}\left(m_{Z}\right)$ and $\delta_{\alpha}$ together with the constraint $\delta_{\alpha}=0.0 \pm 0.1$ [28] by :

$$
\chi_{\mathrm{SM}}^{2}\left(m_{t}, m_{H}, \alpha_{s}, \delta_{\alpha}\right)=\left(\frac{m_{t}-\left\langle m_{t}\right\rangle}{\Delta m_{t}}\right)^{2}+\chi_{H}^{2}\left(m_{H}, \alpha_{s}, \delta_{\alpha}\right)
$$


where

$$
\begin{aligned}
& \left\langle m_{t}\right\rangle=145.2+12.5 \ln \frac{m_{H}}{100}+0.9 \ln ^{2} \frac{m_{H}}{100}-1.9\left(\frac{\alpha_{s}-0.12}{0.01}\right)-4.6\left(\frac{\delta_{\alpha}}{0.10}\right), \\
& \Delta m_{t}=14.6-0.23 \ln \frac{m_{H}}{100}-\left(0.38-0.05 \ln \frac{m_{H}}{100}\right) \frac{m_{t}-150}{10},
\end{aligned}
$$

and

$$
\begin{aligned}
\chi_{H}^{2}\left(m_{H}, \alpha_{s}, \delta_{\alpha}\right)= & 6.11+\left(\frac{\delta_{\alpha}-0.31}{0.43}\right)^{2}+\left(\frac{\alpha_{s}-0.1173+0.005 \delta_{\alpha}}{0.0060}\right)^{2} \\
& -\left(\frac{\alpha_{s}-0.1244+0.025 \delta_{\alpha}}{0.0136}\right) \ln \frac{m_{H}}{100}-\left(\frac{\alpha_{s}-0.1322}{0.0700}\right) \ln ^{2} \frac{m_{H}}{100}+\left(\frac{\delta_{\alpha}}{0.10}\right)^{2} .
\end{aligned}
$$

Here $m_{t}$ and $m_{H}$ are measured in $\mathrm{GeV}$. This parametrization reproduces the exact $\chi^{2}$ within a few $\%$ accuracy in the range $100 \mathrm{GeV}<m_{t}<250 \mathrm{GeV}, 60 \mathrm{GeV}<m_{H}<$ $1000 \mathrm{GeV}$ and $0.10<\alpha_{s}\left(m_{Z}\right)<0.13$. The best-fit value of $m_{t}$ for a given set of $m_{H}, \alpha_{s}$ and $\delta_{\alpha}$ is readily obtained from eq. (5.9b) with its approximate error of (5.9g), mutatis mutandis for $m_{H}$. Due to the quadratic form it is easy to get the $\alpha_{s}$ or $\delta_{\alpha}$ independent results. Also additional constraints on the external parameters $\alpha_{s}$ and $\delta_{\alpha}$, such as those from their improved measurements, can be discussed without difficulty. As explained in section 4.1, the SM does not fit well the ratio $R_{b}$. If we remove from our global fit the data on $R_{b}$, we find that the best-fit $m_{t}$ value above becomes larger by $3.9 \mathrm{GeV}$, almost independent of $m_{H}$ and $\alpha_{s}$, and the $\chi_{\min }^{2}$ decreases by 2.4 .

Fig. 23 displays the overall $\chi^{2}$ of the SM fit, $\chi_{\mathrm{SM}}^{2}$, as function of $m_{t}$ for $m_{H}=60,300$, $1000 \mathrm{GeV}$ and $\alpha_{s}\left(m_{Z}\right)=0.11,0.12,0.13$. Also the uncertainty due to $\delta_{\alpha}$ is shown for three cases, $\delta_{\alpha}=-0.1$ (a), 0 (b), +0.1 (c). The results of the parametrization eq. (5.9) is shown by the dotted line. It is remarkable to see that the present knowledge of $\delta_{\alpha}$ to \pm 0.10 affects the best-fit value of $m_{t}$ by about $5 \mathrm{GeV}$, while the uncertainty in $\alpha_{s}$ of \pm 0.01 affects it by about $2 \mathrm{GeV}$. This observation emphasizes the importance of the asymmetry measurements for the prediction of $m_{t}$ through $\bar{s}^{2}\left(m_{Z}^{2}\right)$, where the dependence on $\delta_{\alpha}$ in the SM is not negligible: see eq. (2.38b). On the other hand, the $\alpha_{s}$-dependence of the fitted $m_{t}$ comes from the constraint due to the $Z$ total width, $\Gamma_{Z}$, which in turn is sensitive to $m_{t}$ mainly through $\bar{g}_{Z}^{2}\left(m_{Z}^{2}\right)$. We come back to this point in the next section when discussing the new left-right asymmetry measurement [31].

In Fig. 24 the overall $\chi^{2}$ is plotted as functions of $m_{H}$ for $m_{t}=120,140,160,180$, $200 \mathrm{GeV}$ and $\alpha_{s}\left(m_{Z}\right)=0.11,0.12,0.13$ setting $\delta_{\alpha}=0$. The dotted lines indicate our approximation $\chi_{\mathrm{SM}}^{2}$ of (5.9). Obviously, the best-fit value of $m_{H}$ depends very sensitively on the $m_{t}$ and $\alpha_{s}$ values. A small value of the Higgs mass is favored for $m_{t}<140 \mathrm{GeV}$, values of a few hundred $\mathrm{GeV}$ for $m_{t}$ around $160 \mathrm{GeV}$ and large values for $m_{t}>180 \mathrm{GeV}$. 
The preference of lighter $m_{H}$ is more pronounced for small $\alpha_{s}$, while heavier $m_{H}$ for larger $\alpha_{s}$. However, the $m_{H}$ dependence of $\chi^{2}$ is very mild and meaningful upper bound on $m_{H}$ can only be obtained for small $\alpha_{s}$ and small $m_{t}$. The upper and lower bounds on $m_{H}$ will be discussed more quantitatively in section 6 after inclusion of the new left-right asymmetry data 31].

For given $m_{t}$ and $m_{H}$ the QCD coupling $\alpha_{s}\left(m_{Z}\right)$ may be extracted within the SM from the electroweak data alone with the result :

$$
\begin{aligned}
\alpha_{s} & =\left\langle\alpha_{s}\right\rangle \pm 0.0060, \\
\left\langle\alpha_{s}\right\rangle & =0.1165-0.00085\left(\frac{m_{t}}{100}\right)^{2}+0.00031\left(\ln \frac{m_{H}}{100}+2.6\right)^{2}-0.0006 \frac{\delta_{\alpha}}{0.10},
\end{aligned}
$$

where $m_{t}$ and $m_{H}$ are measured in GeV. The above parametrization reproduces well the $\alpha_{s}$ dependence of the $\chi^{2}$ function (5.9) in the range $100 \mathrm{GeV}<m_{t}<200 \mathrm{GeV}$ and $60 \mathrm{GeV}<m_{H}<1000 \mathrm{GeV}$. The error on $\alpha_{s}$ determined from the electroweak data is found to be approximately 0.0060 , almost independently of the assumed $m_{t}, m_{H}$ and $\delta_{\alpha}$, while the mean value $\left\langle\alpha_{s}\right\rangle$ is slightly sensitive to them;

$$
\left\langle\alpha_{s}\right\rangle= \begin{cases}0.1159 & \text { for } \quad\left(m_{t}, m_{H}\right)=(150,60) \mathrm{GeV} \\ 0.1153 & \text { for } \quad\left(m_{t}, m_{H}\right)=(175,60) \mathrm{GeV} \\ 0.1145 \text { for } \quad\left(m_{t}, m_{H}\right)=(200,60) \mathrm{GeV} \\ 0.1220 \text { for } \quad\left(m_{t}, m_{H}\right)=(150,1000) \mathrm{GeV} \\ 0.1214 \text { for } \quad\left(m_{t}, m_{H}\right)=(175,1000) \mathrm{GeV} \\ 0.1206 \text { for } \quad\left(m_{t}, m_{H}\right)=(200,1000) \mathrm{GeV}\end{cases}
$$

for $\delta_{\alpha}=0$. There is a tendency in the SM fit to prefer larger $\alpha_{s}$ for larger $m_{H}$.

Furthermore, if all radiative effects are assumed to be dominated by the SM contributions, the present electroweak data have some sensitivity to the parameter $\delta_{\alpha} \equiv$ $1 / \bar{\alpha}\left(m_{Z}^{2}\right)-128.72$. By excluding the last term in eq. (5.9d), $\left(\delta_{\alpha} / 0.1\right)^{2}$ [28], the electroweak data alone provide the constraint :

$$
\begin{aligned}
\delta_{\alpha} & =\left\langle\delta_{\alpha}\right\rangle \pm 0.24, \\
\left\langle\delta_{\alpha}\right\rangle & =0.010-0.139 \frac{m_{t}-150}{10}+0.246 \ln \frac{m_{H}}{100}-0.112 \frac{\alpha_{s}-0.12}{0.01},
\end{aligned}
$$

where $m_{t}$ and $m_{H}$ are measured in $\mathrm{GeV}$. The above parametrization is valid in the range $120 \mathrm{GeV}<m_{t}<200 \mathrm{GeV}, 60 \mathrm{GeV}<m_{H}<1000 \mathrm{GeV}$ and $0.11<\alpha_{s}<0.13$. For some representative $m_{t}$ and $m_{H}$ values the exact evaluation of the $\chi^{2}$ function leads to :

$$
\left\langle\delta_{\alpha}\right\rangle=\left\{\begin{aligned}
&-0.09 \text { for } \quad\left(m_{t}, m_{H}\right)=(150,60) \mathrm{GeV} \\
&-0.45 \text { for } \quad\left(m_{t}, m_{H}\right)=(175,60) \mathrm{GeV} \\
&-0.87 \text { for } \quad\left(m_{t}, m_{H}\right)=(200,60) \mathrm{GeV} \\
& 0.59 \text { for } \quad\left(m_{t}, m_{H}\right)=(150,1000) \mathrm{GeV} \\
& 0.25 \text { for } \quad\left(m_{t}, m_{H}\right)=(175,1000) \mathrm{GeV} \\
&-0.12 \text { for } \quad\left(m_{t}, m_{H}\right)=(200,1000) \mathrm{GeV}
\end{aligned}\right.
$$


for $\alpha_{s}=0.12$. The above fit is consistent with the direct measurement $\left[\delta_{\alpha}\right]_{\mathrm{SM}} \approx \delta_{\text {had }}=$ $0 \pm 0.10$ when $m_{t}$ and $m_{H}$ are in the preferred range in Fig. 22. This confirms the importance of the direct $\delta_{\text {had }}$ measurement in constraining the model parameters from the electroweak precision measurements.

Considering the $\chi_{\min }^{2}$ per degree of freedom (see parametrization (5.9) and Figs. 22 24) the SM predictions provide a good description of the data over a still wide range of $m_{t}$ and $m_{H}$ for the values of $\alpha_{s}$ and $\delta_{\alpha}$ in the ranges: $0.11 \lesssim \alpha_{s} \lesssim 0.13$ and $-0.1 \lesssim \delta_{\alpha} \lesssim 0.1$. In conclusion, the analysis of the present precision experiments does not show a signal of new physics beyond the SM.

\section{Discussion}

In this section, the consequences of the update of LEP data, the new precision measurement of the left-right asymmetry at SLC [31] and the impact of a direct top mass measurement are considered. Finally, the predictions of all electroweak observables within the SM are discussed.

\subsection{Update of LEP data}

Recently the LEP Electroweak Working Group has published a report 91 summarizing the combination of preliminary LEP data for the 1994 La Thuile and Moriond conferences. During 1993 the four LEP experiments have performed a high precision scan roughly $1.8 \mathrm{GeV}$ above and below the $Z$ resonance and within $200 \mathrm{MeV}$ of $m_{Z}$. The new $Z$ shape parameters agree with the ones quoted in section 4.1 within one standard deviation. The $Z$ mass moved to $91.1895 \pm 0.0044 \mathrm{GeV}$ with improved uncertainty. Changing of the 'constant' $m_{Z}$ from $91.187 \mathrm{GeV}$ to $91.1895 \mathrm{GeV}$ does not lead to noticeable effects in the analysis. The total $Z$ width increased to $2.4969 \pm 0.0038 \mathrm{GeV}$ with considerably reduced uncertainty, also the forward-backward lepton asymmetry increased to $0.0170 \pm$ 0.0016. Other parameters, $\sigma_{h}^{0}, R_{\ell}, R_{b}$, have changed very little. The correlations in the $Z$ line-shape parameter fit have become slightly smaller.

For the time being no attempt has been made to incorporate the updated values, since the analyses of the 1993 data are still preliminary.

\subsection{The new left-right asymmetry data at SLC}

As emphasized in sections 3 and 4, the left-right asymmetry as well as the other asymmetry measurements at LEP have the advantage of determining the universal parameter $\bar{s}^{2}\left(m_{Z}^{2}\right)$ almost independently of the other form factors, $\bar{g}_{Z}^{2}\left(m_{Z}^{2}\right)$ and $\bar{\delta}_{b}\left(m_{Z}^{2}\right)$, and almost 
unaffected by uncertainty in $\alpha_{s}$. Since the parameter $\bar{s}^{2}\left(m_{Z}^{2}\right)$ is directly related to the $\overline{\mathrm{MS}}$ coupling $\hat{s}^{2}(\mu)$, these asymmetry measurements are particularly important for the GUT studies.

The new measurement of the left-right asymmetry [31,

$$
A_{\mathrm{LR}}^{0}=0.1656 \pm 0.0076,
$$

implies

$$
\bar{s}^{2}\left(m_{Z}^{2}\right)=0.2282 \pm 0.0010 .
$$

This value is 2.5 standard deviations smaller than (4.5b). Excluding the possibility of a shift caused by a systematic effect this measurement may be considered as a statistical fluctuation and then be combined with the other asymmetry data on the $Z$-pole, that is, the lepton $(e, \mu, \tau)$ forward-backward asymmetry [26], the $\tau$ polarization asymmetry [26], the left-right asymmetry [31] and the quark $(b, c)$ forward-backward asymmetries [26], as well as with the old left-right asymmetry data from SLD [90]. The result is

$$
\bar{s}^{2}\left(m_{Z}^{2}\right)=0.2302 \pm 0.0005 .
$$

The new average (denoted by "ALL") is shown in Fig. 25 together with the individual contributions. [ Note that $\bar{s}^{2}\left(m_{Z}^{2}\right)$ derived from the $\tau$ forward-backward asymmetry is as small as (6.2) from the new left-right asymmetry. Although the inclusion of the new left-right asymmetry lowers the $\bar{s}^{2}\left(m_{Z}^{2}\right)$ fit value by about 1.5 standard deviations, the quality of the fit ( $\chi^{2}=6.6$ for 5 degrees of freedom) does not indicate an inconsistency with the other data, as may be seen also from the histogram of the distribution in the figure.

With the proviso of excluding a shift due to systematic error sources we include the data (6.1) into our global analysis, and discuss its effect by comparing the results with those obtained in section 4 . The 3 parameter fit to the $Z$ parameters only gives

$$
\begin{aligned}
\bar{g}_{Z}^{2}\left(m_{Z}^{2}\right)= & 0.5538-0.00031 \frac{\alpha_{s}-0.12}{0_{0} 01} \pm 0.0017 \\
\bar{s}^{2}\left(m_{Z}^{2}\right)= & 0.2303+0.00006 \frac{\alpha_{s}-0.12}{0.01} \pm 0.0005 \\
\bar{\delta}_{b}\left(m_{Z}^{2}\right)= & -0.0071-0.00432 \frac{\alpha_{s}-0.12}{0.01} \pm 0.0035 \\
& \chi_{\min }^{2}=5.78+\left(\frac{\alpha_{s}-0.1000}{0.0127}\right)^{2} .
\end{aligned}
$$

The result is shown in the $\left(\bar{s}^{2}\left(m_{Z}^{2}\right), \bar{g}_{Z}^{2}\left(m_{Z}^{2}\right)\right)$ plane by the thick lines of Fig. 26(a) for three values $\alpha_{s}=0.11,0.12,0.13$ along with the old fits (thin lines) copied from Fig. 15.

\footnotetext{
${ }^{6}$ In Fig. 25 and in the following analysis, we use the combined result of [90] and [31] as the data for $A_{\mathrm{LR}}^{0}: A_{\mathrm{LR}}^{0}=0.1637 \pm 0.0075$ gives $\bar{s}^{2}\left(m_{Z}^{2}\right)=0.2284 \pm 0.0010$.
} 
The SM prediction for $\delta_{\alpha}=0$ is also shown in the range $100 \mathrm{GeV}<m_{t}<240 \mathrm{GeV}$ and $1 \mathrm{GeV}<m_{H}<1000 \mathrm{GeV}$. It can be seen that the new $A_{\mathrm{LR}}$ measurement by itself implies large $m_{t}\left(m_{t} \gtrsim 200 \mathrm{GeV}\right)$ for $m_{H}>50 \mathrm{GeV}$. The combined fit, however, favors $m_{t} \sim 180 \mathrm{GeV}$ for $m_{H} \sim 100 \mathrm{GeV}$. The remaining two parameters $\bar{g}_{Z}^{2}\left(m_{Z}^{2}\right)$ and $\bar{\delta}_{b}\left(m_{Z}^{2}\right)$ are less affected. The $\chi_{\min }^{2}$ per degree of freedom is $8.4 / 6$ for $\alpha_{s}=0.12$, which is fine.

Next, the 4-parameter fit in terms of $S, T, U$ and $\bar{\delta}_{b}$ is performed analogously to the one in section 5.3. Combining the above result with eq. (4.35) from the low energy neutral current experiments and eq. (4.39) from the $W$ mass measurements leads to

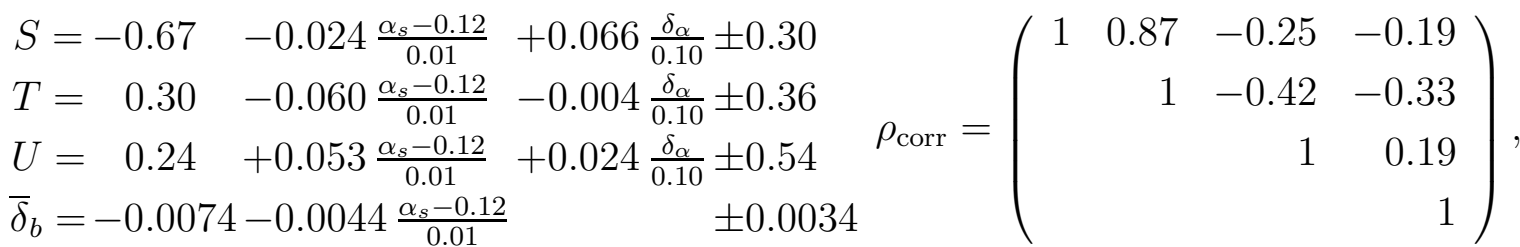

$$
\begin{aligned}
& \chi_{\min }^{2}=8.60+\left(\frac{\alpha_{s}-0.0998}{0.0126}\right)^{2}+\left(\frac{\delta_{\alpha}}{0.10}\right)^{2},
\end{aligned}
$$

where $m_{t}=175 \mathrm{GeV}$ and $m_{H}=100 \mathrm{GeV}$ are used to calculate SM running of the form factors between $q^{2}=0$ and $q^{2}=m_{Z}^{2}$. Fig. 26(b) shows the 1- $\sigma$ contours in the $(S, T)$ plane for the three values $\alpha_{s}=0.11,0.12,0.13$ for $\delta_{\alpha}=0$. The old fits (5.8) are also shown by thin lines. The results are insensitive to the above $\left(m_{t}, m_{H}\right)$ values assumed in the running of the charge form factors in the region $m_{t}>100 \mathrm{GeV}$ and $m_{H}>50 \mathrm{GeV}$, although they are considerably modified for $m_{H} \lesssim 50 \mathrm{GeV}$ (see Fig. 18). It is worth noting this qualitative difference between the fit to $\bar{s}^{2}\left(m_{Z}^{2}\right)$ and $\bar{g}_{Z}^{2}\left(m_{Z}^{2}\right)$ and that to $S$ and $T$. As a matter of fact, the experiments on the $Z$ resonance are far more precise than those from the low energy neutral current experiments implying that the global fit to all the electroweak measurements in the neutral current sector measures essentially $\bar{s}^{2}\left(m_{Z}^{2}\right)$ and $\bar{g}_{Z}^{2}\left(m_{Z}^{2}\right)$. In the SM the two charge form factors can be calculated for arbitrary $m_{t}$ and $m_{H}$, as shown in the figure for $m_{t}=100-240 \mathrm{GeV}$ and $m_{H}=1-1000 \mathrm{GeV}$. On the other hand, in our definition, the $T$ parameter determines $\bar{g}_{Z}^{2}(0)$ rather than $\bar{g}_{Z}^{2}\left(m_{Z}^{2}\right)$. Hence, only if the running of the $\bar{g}_{Z}^{2}\left(q^{2}\right)$ between $q^{2}=0$ and $q^{2}=m_{Z}^{2}$ is small, can we make the global fit to the $S, T$ parameters. For this reason we restrict the SM predictions to the region $m_{H}=50-1000 \mathrm{GeV}$ in the $(S, T)$ figure. It is remarkable that the electroweak data including the new left-right asymmetry measurement clearly favor negative $S$, thus putting severe constraints on technicolor models 迎. Note that in the $(S, T)$ plane only the $S$ parameter is strongly affected by the new $A_{\mathrm{LR}}$ data, while the $T$ parameter is constrained, independent of the $S$ parameter, by $\bar{g}_{Z}^{2}\left(m_{Z}^{2}\right)$ from $\Gamma_{Z}$.

Next, the impact of the left-right asymmetry measurement on the SM fit is discussed using all electroweak data. Fig. 27 shows the results of the SM fit in the $\left(m_{t}, m_{H}\right)$ plane 
for $\alpha_{s}\left(m_{Z}\right)=0.11,0.12,0.13$, and for $\delta_{\alpha}=-0.1(\mathrm{a}), 0$ (b), and +0.1 (c). The contours of $\chi^{2}=\chi_{\min }^{2}+1$ and $\chi^{2}=\chi_{\min }^{2}+4.61$ are shown by thick lines. The minima of $\chi^{2}$ in the figure are marked by crosses : $12.1,11.4,15.7$ for $\alpha_{s}=0.11,0.12,0.13$, respectively, for $\delta_{\alpha}=0$. The $1-\sigma$ contour for each $\alpha_{s}$ value is now clearly outside the physical region allowed by the direct Higgs searches at LEP $\left(m_{H}>63 \mathrm{GeV}\right.$, denoted by "LEP limit" in the figures), although the $m_{H}$ dependence of the $\chi^{2}$ is very mild for $\alpha_{s} \gtrsim 0.12$. The result favoring a light Higgs boson reflects the fact that the new left-right asymmetry measurement shifts the $S$ parameter to negative values.

Finally, the status of the SM fit is studied in detail as in section 5. To this end the representation of the $\chi^{2}$ of the SM fit including the new left-right asymmetry data is obtained (analogous to section 5.4) :

$$
\chi_{\mathrm{SM}}^{2}\left(m_{t}, m_{H}, \alpha_{s}, \delta_{\alpha}\right)=\left(\frac{m_{t}-\left\langle m_{t}\right\rangle}{\Delta m_{t}}\right)^{2}+\chi_{H}^{2}\left(m_{H}, \alpha_{s}, \delta_{\alpha}\right),
$$

where

$$
\begin{aligned}
& \left\langle m_{t}\right\rangle=162.2+12.6 \ln \frac{m_{H}}{100}+0.8 \ln ^{2} \frac{m_{H}}{100}-1.2\left(\frac{\alpha_{s}-0.12}{0.01}\right)-4.8\left(\frac{\delta_{\alpha}}{0.10}\right), \\
& \Delta m_{t}=12.0-0.09 \ln \frac{m_{H}}{100}-\left(0.31-0.05 \ln \frac{m_{H}}{100}\right) \frac{m_{t}-175}{10},
\end{aligned}
$$

and

$$
\begin{aligned}
\chi_{H}^{2}\left(m_{H}, \alpha_{s}, \delta_{\alpha}\right)= & 9.56+\left(\frac{\delta_{\alpha}-0.75}{0.39}\right)^{2}+\left(\frac{\alpha_{s}-0.1164+0.005 \delta_{\alpha}}{0.0060}\right)^{2} \\
& -\left(\frac{\alpha_{s}-0.1365+0.030 \delta_{\alpha}}{0.0144}\right) \ln \frac{m_{H}}{100}-\left(\frac{\alpha_{s}-0.1255}{0.0639}\right) \ln ^{2} \frac{m_{H}}{100}+\left(\frac{\delta_{\alpha}}{0.10}\right)^{2} .
\end{aligned}
$$

Fig. 28 (in analogy to the previous results of Fig. 23) shows the total $\chi^{2}$ of the SM fit as functions of $m_{t}$ for $m_{H}=60,300,1000 \mathrm{GeV}$ and $\alpha_{s}\left(m_{Z}\right)=0.11,0.12,0.13$. The uncertainty $\delta_{\alpha}$ is shown for three cases : $\delta_{\alpha}=-0.1$ (a), 0 (b), +0.1 (c). The dotted lines are obtained by the approximate formulae (6.6). It is obvious from Fig. 28 and Fig. 23, or from eq. (6.6b) and eq. (5.9b), that the best-fit value of $m_{t}$ is shifted by about $+17 \mathrm{GeV}$ for given $m_{H}, \alpha_{s}$ and $\delta_{\alpha}$ values. Here again the uncertainty of $\delta_{\alpha}$ is important for the top mass prediction, as observed from (6.6b) and Fig. 28: $\delta_{\alpha}= \pm 0.1$ causes a shift $\mp 5 \mathrm{GeV}$ in the best-fit value $\left\langle m_{t}\right\rangle$. The $\alpha_{s}$-dependence of the $\left\langle m_{t}\right\rangle$ values is considerably weakened.

Fig. 29 (in correspondence to Fig. 24 in the previous fit) shows the total $\chi^{2}$ of the SM fit including the new left-right asymmetry data [31], as functions of $m_{H}$ for $m_{t}=100$ $200 \mathrm{GeV}$. Three $\alpha_{s}$ cases are displayed; $\alpha_{s}\left(m_{Z}\right)=0.11$ (a), 0.12 (b), and 0.13 (c), all for $\delta_{\alpha}=0$. The dotted lines show our approximation (6.6), valid only in the 'physical' 
region $63 \mathrm{GeV}<m_{H}<1000 \mathrm{GeV}$. As seen, the best-fit value of $m_{H}$ is as low as $10 \mathrm{GeV}$ for $m_{t} \lesssim 150 \mathrm{GeV}$, while it increases with $m_{t}$ for $m_{t} \gtrsim 150 \mathrm{GeV}$. This trend can also be appreciated from the global fit of Fig. 26(a) in the $\left(\bar{s}^{2}\left(m_{Z}^{2}\right), \bar{g}_{Z}^{2}\left(m_{Z}^{2}\right)\right)$ plane.

\subsection{The impact of the top mass measurement}

The top quark searches of the two collaborations CDF and D0 at the Tevatron entered in their decisive phase [105, 106]. The range of values for the top quark mass coming out of the fits to the electroweak precision data is within reach for direct observation in the detectors at the Tevatron. In view of the recent publication by the CDF collaboration [106] it is instructive to examine the impact of the constraint

$$
m_{t}=174 \pm 16 \mathrm{GeV} .
$$

First, the $m_{t}$-dependence of the global fit to the electroweak data in terms of the charge form factors $\bar{s}^{2}\left(m_{Z}^{2}\right)$ and $\bar{g}_{Z}^{2}\left(m_{Z}^{2}\right)$ is considered, now assuming SM dominance to the $\bar{\delta}_{b}\left(m_{Z}^{2}\right)$ form factor. Using the $Z$ parameters including the new $A_{\mathrm{LR}}$ measurement [31] one obtains

$$
\begin{aligned}
& \left.\begin{array}{l}
\bar{g}_{Z}^{2}\left(m_{Z}^{2}\right)=0.55430-0.00109 \frac{\alpha_{s}-0.12-0.0023 \frac{m_{t}-174}{16}}{0.01} \pm 0.00156 \\
\bar{s}^{2}\left(m_{Z}^{2}\right)=0.23023+0.00016 \frac{\alpha_{s}-0.12-0.0021 \frac{m_{t}-174}{16}}{0.01} \pm 0.00054
\end{array}\right\} \rho_{\text {corr }}=0.19, \\
& \chi_{\min }^{2}=6.86+\left(\frac{m_{t}-90}{60}\right)^{2}+\left(\frac{\alpha_{s}-0.1187-0.0022 \frac{m_{t}-174}{16}}{0.0068}\right)^{2},
\end{aligned}
$$

which is a good approximation in the region $150 \mathrm{GeV}<m_{t}<200 \mathrm{GeV}$. Here the errors and the correlations are almost independent of the $m_{t}$ value. The fit to all electroweak data gives

$$
\begin{aligned}
& \left.\begin{array}{l}
S=-0.62-0.097 \frac{\alpha_{s}-0.12-0.0022 \frac{m_{t}-174}{16}}{0.01}+0.066 \frac{\delta_{\alpha}}{0.10} \pm 0.30 \\
T=0.39-0.214 \frac{\alpha_{s}-0.12-0.0022 \frac{m_{t}-174}{16}}{0.01}-0.004 \frac{\delta_{\alpha}}{0.10} \pm 0.34 \\
U=0.17+0.182 \frac{\alpha_{s}-0.12-0.0022 \frac{m_{t}-174}{16}}{0.01}+0.023 \frac{\delta_{\alpha}}{0.10} \pm 0.53
\end{array}\right\} \rho_{\text {corr }}=\left(\begin{array}{rrr}
1 & 0.87 & -0.22 \\
1 & -0.38 \\
& 1
\end{array}\right), \\
& \chi_{\min }^{2}=9.58+\left(\frac{m_{t}-84}{63}\right)^{2}+\left(\frac{\alpha_{s}-0.1185-0.0022 \frac{m_{t}-174}{16}}{0.0067}\right)^{2}+\left(\frac{\delta_{\alpha}}{0.10}\right)^{2} .
\end{aligned}
$$

The appearance of essentially the same combination

$$
\alpha_{s}-0.12-0.0022 \frac{m_{t}-174}{16}
$$

in eqs. (6.8) and (6.9) is the expected consequence of the strong correlation between $\bar{\delta}_{b}\left(m_{Z}^{2}\right)$ and $\alpha_{s}$ as discussed in detail in section 4. 
Next, the above constraint on the top quark mass (6.7) is imposed on the $\chi^{2}$ function of the SM fit in the previous subsection. The result displayed in Fig. 31 shows the improvement over Fig. 27. Now, light Higgs boson masses are moderately favored, as a consequence of the constraint (6.7) being somewhat larger than the best-fit value of $m_{t}$ obtained by freely fitting the two parameters, $m_{t}$ and $m_{H}$ without the $m_{H}$ constraint from LEP.

It is instructive to anticipate the impact a precise measurement of the top mass would have in the context of the present electroweak data. The top quark mass is expected to be measured eventually with an uncertainty of about $5 \mathrm{GeV}$ at Tevatron by the end of this decade [107], which may be improved to about $3 \mathrm{GeV}$ at an upgraded Tevatron [108]. The uncertainty is expected to be reduced by an order of magnitude to a few hundred $\mathrm{MeV}$ at next linear $e^{+} e^{-}$colliders [109]. The top mass acts then like an external parameter and the only remaining free parameter is the Higgs mass. Fig. 32 shows the 95\% CL constraints for three values of $\alpha_{s}\left(m_{Z}\right)=0.11,0.12,0.13$, and for $\delta_{\alpha}=0$. For small $m_{t}$ values, rather strict upper bounds on $m_{H}$ are found. On the other hand no strict upper bound is obtained for $m_{t} \gtrsim 180 \mathrm{GeV}$. In the region $160 \mathrm{GeV}<m_{t}<190 \mathrm{GeV}$, the upper bound on $m_{H}$ at the $95 \%$ CL is approximately expressed as

$$
\ln \frac{m_{H}}{100}<\left\{\begin{array}{lll}
1.20+1.12 \frac{m_{t}-174}{16} & \text { for } & \alpha_{s}=0.11 \\
1.55+1.25 \frac{m_{t}-174}{16} & \text { for } & \alpha_{s}=0.12 \\
1.95+1.45 \frac{m_{t}-174}{16} & \text { for } & \alpha_{s}=0.13
\end{array},\right.
$$

where $m_{t}$ and $m_{H}$ are measured in $\mathrm{GeV}$. The upper bound is lower for smaller $m_{t}$. Since these bounds are very sensitive to the $m_{t}$ value as well as the assumed $\alpha_{s}$ value, further accurate measurements of $m_{t}$ are needed to obtain more stringent limits on $m_{H}$. Nevertheless, it is remarkable that the constraint on the top quark mass (6.7) would favor a relatively light Higgs boson, $m_{H}=O(100 \mathrm{GeV})$, which may exist in the minimal SUSYSM. Also, the direct $m_{H}$ bound from LEP [101] $m_{H}>63 \mathrm{GeV}(95 \% \mathrm{CL})$ implies that the top quark should be heavier than about $145 \mathrm{GeV}$, since otherwise the Higgs boson should have been discovered at the $95 \% \mathrm{CL}$. This lower $m_{t}$ bound changes by about $\mp 5 \mathrm{GeV}$ for $\delta_{\alpha}= \pm 0.1$.

One comment is in order. Though our approximate formulae of the $\chi^{2}$ for the SM fit, (6.6), reproduce the exact result within about 1\% accuracy in the Higgs mass range $63 \mathrm{GeV}<m_{H}<1000 \mathrm{GeV}$ as seen Figs. 28 29, one should not use them in finding the confidence levels of $m_{H}$ for small $m_{t}$, since the neighborhood of the minimum of the $\chi^{2}$ is outside the above range, where the exact $\chi^{2}$ and the approximate formulae are fairly different as seen from Fig. 29. 


\subsection{Summary of the data and the SM fit}

Table 7 collects the complete list of all input data (except for $\alpha, G_{F}$ and $m_{Z}$ ) and the corresponding minimal SM predictions for several sets of $\left(m_{t}, m_{H}, \alpha_{s}\right)$ values. The total $\chi^{2}$ of each sector is also given in the table. The correlations between the errors (given in the text) are properly taken into account. The numbers demonstrate that the present electroweak experiments are well described by the SM, perhaps except for a combination of a light top and a heavy Higgs, see the case $\left(m_{t}, m_{H}\right)=(150,1000) \mathrm{GeV}$ in the last column of the table. Its total $\chi^{2}$ at $\alpha_{s}=0.12$ is 30.22 for 19 data points, whose $\chi^{2}$-probability corresponds to $95 \%$. In Table 7 also the results of two approximations are listed. The 'no-EW' column is obtained by dropping all electroweak corrections to the two-point functions $(S=T=U=0)$ as well as vertex/box corrections $\left(\bar{\delta}_{G}=\bar{\delta}_{b}=\Gamma_{i}=B_{i j}=0\right)$, while retaining the QED running of the charge form factors $\bar{\alpha}\left(q^{2}\right)$ and $\bar{s}^{2}\left(q^{2}\right) / \bar{\alpha}\left(q^{2}\right)$ due to light particles (excluding the $W$ and $t$ contributions). The 'IBA' column shows the result of the improved Born approximation, where all the gauge boson propagator corrections are retained and hence all the four charge form factors are kept exact, but all vertex/box corrections $\left(\bar{\delta}_{b}=\Gamma_{i}=B_{i j}=0\right)$ dropped, except for $\bar{\delta}_{G}$ in the $\mu$ decay.

It is amazing to note that the 'no-EW' hypothesis is, from a statistical point of view, not completely unacceptable. The comparison between the 'no-EW' and the 'IBA' hypothesis is surprising, since in the 'IBA' prediction all the most important electroweak corrections are supposed to be contained, including the dominant $m_{t}^{2}$ corrections in the $T$ parameter. It is even more striking, if $\bar{\delta}_{G}$ in IBA is set to 0 (this may be called a genuine IBA), to obtain $\bar{s}^{2}\left(m_{Z}^{2}\right)=0.2286$ for $m_{t}=175 \mathrm{GeV}$ and the total $\chi^{2}$ jumps nearly to 100 . The measurement of the $Z$ parameters are equally well described by the 'no-EW' and the full calculation for $m_{t}=175 \mathrm{GeV}$. This confirms the observation of ref. [64, 110 that there is no evidence of the genuine electroweak correction in the present electroweak precision experiments. As explained in sections 2.3 and 5.3, this is because of the accidental cancellation between the propagator corrections and the remaining vertex/box corrections. The no-EW calculation for all the asymmetries on the $Z$-pole give almost the same values with the predictions of the exact calculation for $m_{t}=175 \mathrm{GeV}$ and $m_{H}=100 \mathrm{GeV}$. As discussed in section 4.1, $R_{b}$ also gives a large contribution to $\chi^{2}$ in the full calculation. For a large top quark mass, the $Z b_{L} b_{L}$ vertex from factor $\bar{\delta}_{b}\left(m_{Z}^{2}\right)$ decreases (see Fig. 1), and hence it gives smaller $R_{b}$. For this reason the present data of $R_{b}$ agree better with the no-EW and the IBA calculations, where $\bar{\delta}_{b}\left(m_{Z}^{2}\right)$ is set to 0 .

The most significant differences between the no-EW prediction and the full SM predictions in $\left(m_{t}=175 \mathrm{GeV}, m_{H}=100 \mathrm{GeV}\right)$ column appear actually in the predictions for the low energy $\nu_{\mu}-q$ scattering and the atomic parity violation experiments. When 
evaluating the no-EW and IBA predictions, all the external photonic corrections and the tree-level propagator effects are retained, as explained in section 3.2. The difference between the full SM predictions and the no-EW or IBA predictions is mainly caused by the absence of the $W W$ box contribution in the latter.

Another significant difference appears in the predictions for $m_{W}$, where the no-EW prediction $(79.95 \mathrm{GeV})$ is much smaller than the observed value, $80.24 \pm 0.16 \mathrm{GeV}$. This observation has also been made in refs. [110 112]. In contrast to the low energy neutral current experiments above, the difference here is due to $S$ and $U$ contributing to $m_{W}$ proportional to $-0.294 S+0.332 U$ (c.f. eq. (3.100)). For instance, the full SM for $m_{t}=$ $175 \mathrm{GeV}$ and $m_{H}=100 \mathrm{GeV}$ predicts $S=-0.2323$ and $U=0.3577$, which implies for $m_{W}$ a shift by $0.19 \mathrm{GeV}$ corresponding to more than one standard deviation.

Finally, Fig. 33 shows separately for each sector the $\chi^{2}$ of the SM fit as functions of $m_{H}$ for $m_{t}=100-200 \mathrm{GeV}$. In all sectors, the preferred Higgs mass range is strongly correlated with the assumed top mass. For $m_{t}=170-180 \mathrm{GeV}$, a light Higgs boson is favored by the $Z$ parameter measurements and by the low energy neutral current experiments, while the data of $m_{W}$ alone prefer a rather heavy Higgs boson. Although the overall trend of the total $\chi^{2}$ shown in Fig. 29 is dominated by the contribution from the $\mathrm{Z}$ parameter measurements, also the $W$ mass measurement plays an important role for some $m_{t}, m_{H}$ ranges. For instance, a relatively light Higgs boson $\left(m_{H} \lesssim 100 \mathrm{GeV}\right)$ appears incompatible with a heavy top quark $\left(m_{t} \sim 200 \mathrm{GeV}\right)$ by the $m_{W}$ measurement alone.

\section{Conclusions}

A novel method to confront electroweak data with theory at the quantum level has been proposed and a comprehensive analysis has been carried out. The electroweak observables were first expressed in terms of model-independent parameters, which in turn were expressed in terms of $S$-matrix elements of processes with four light fermions and factorized into the short-distance part and the part related with the external QED/QCD corrections for neutral current processes. Only two quantities, the Fermi coupling constant $G_{F}$ and the $W$ mass are considered for charged current processes. Since all electroweak observables were expressed in terms of helicity amplitudes, they can be evaluated in an arbitrary model on and off the $Z$ resonance. Our formalism is hence useful to study effects of tree-level deviations from the SM, arising, for instance, from an additional $Z$ boson. After careful evaluation of the external QED/QCD corrections, the theoretical predictions were confronted with experiment in three steps of increasing theoretical stringency. First, in the class of theories respecting the electroweak gauge group $\mathrm{SU}(2)_{\mathrm{L}} \times \mathrm{U}(1)_{\mathrm{Y}}$ broken spontaneously to $\mathrm{U}(1)_{\mathrm{EM}}$ the radiative effects were classified into process-independent and 
process-dependent ones. Apart from the $Z b_{L} b_{L}$ vertex, all vertex and box corrections were assumed to be given by the SM, while new physics contributions were studied in the most general way by four universal charge form factors. Next, by assuming the running of the charge form factors to be governed by SM physics alone, the electroweak parameters $S$, $T, U$ were determined. Finally, the SM itself was confronted with experiments.

It was our aim to render this analysis as transparent as possible by developing the theoretical formalism in full detail and by presenting the results in figures and parametrizations in a form useful for appreciating consequences of future improvements in the experimental data.

The analysis proceeded in two steps. First, the information in the whole body of electroweak precision data has been condensed in the 9 electroweak parameters : $m_{W}$ and $m_{Z}, \bar{e}^{2}(0), \bar{s}^{2}(0), \bar{g}_{Z}^{2}(0)$ and $\bar{g}_{W}^{2}(0), \bar{s}^{2}\left(m_{Z}^{2}\right), \bar{g}_{Z}^{2}\left(m_{Z}^{2}\right)$ and $\bar{\delta}_{b}^{2}\left(m_{Z}^{2}\right)$. At the present time no direct information exists for $\bar{e}^{2}\left(m_{Z}^{2}\right)$. In order to keep the analysis flexible $\bar{e}^{2}\left(m_{Z}^{2}\right)$ and also the QCD coupling constant $\alpha_{s}$ have been treated as external parameters in the fit procedure. Second, this universal set of quantities with the complete covariance matrix has been interpreted within the electroweak theory at three qualitatively distinct levels.

The main result is that the data can be consistently interpreted at all levels, in particular there is nowhere evidence against the SM. This conclusion is not affected, when the new precision measurements of the left-right asymmetry from SLD [31] is included. The fits to the universal charge form factors or that to the universal $S, T, U$ parameters work well and do not hint at a violation of the $\mathrm{SU}(2)_{\mathrm{L}} \times \mathrm{U}(1)_{\mathrm{Y}}$ universality, nor at an anomalously large non-standard vertex/box corrections. Generally speaking, the inclusion of the SM vertex/box corrections improves the fit to the data, while the improved Born approximation gives a poor fit to experiments. The ratio $R_{b} \equiv \sigma_{b}^{0} / \sigma_{h}^{0}$ measured by the LEP experiments turned out to be in poor agreement with the large $Z b_{L} b_{L}$ vertex correction predicted by the SM. The fit to the $S, T, U$ parameters gives us information on spontaneous symmetry breaking. The $T$ parameter is essentially determined by the charge form factor $\bar{g}_{Z}^{2}\left(m_{Z}^{2}\right)$, and positive value is favored. The $S$ parameter is then fixed mainly via $\bar{s}^{2}\left(m_{Z}^{2}\right)$, and hence its best-fit value is affected by the asymmetry data. A negative $S$ value is favored by the new left-right asymmetry from SLD, and the naive technicolor models are disfavored 困. Due to strong correlation between the fitted $S$ and $T$ values, the region of the $(S, T)$ plane with relatively large $S$ and $T(-0.3 \lesssim S \lesssim-0.1$ and $0.5 \lesssim T \lesssim 1)$ is consistent with the SM prediction for $150 \mathrm{GeV} \lesssim m_{t} \lesssim 200 \mathrm{GeV}$ and $50 \mathrm{GeV} \lesssim m_{H} \lesssim 200 \mathrm{GeV}$ (see Figs. 26(b) and 30(b)). The $U$ parameter is measured only via $\bar{g}_{W}^{2}(0)$, and it is consistent with zero.

The analysis showed that the experimental precision required to detect a deviation 
from the SM is still insufficient. For instance, the running of the charge form factors can be tested presently only for $\bar{s}^{2}\left(q^{2}\right)$ and $\bar{g}_{Z}^{2}\left(q^{2}\right)$ and is limited by the precision of the low energy neutral current experiments. Nevertheless, the data are precise enough to show that their consistent description within the SM is only guarantied, if the top quark mass exceeds about $145 \mathrm{GeV}$. This low mass bound of $m_{t}$ is nearly independent of $\alpha_{s}$, but changes by about $\mp 5 \mathrm{GeV}$ due to the uncertainty \pm 0.1 in the hadronic vacuum polarization contribution to $\delta_{\alpha} \equiv 1 / \bar{\alpha}\left(m_{Z}^{2}\right)-128.72$. Note that the SM top-bottom contribution to the form factors have been calculated by using the $O\left(\alpha \alpha_{s}\right)$ two-loop formula [54 56]. Perturbative $t \bar{t}$ threshold effects 60 62 will affect these corrections, and the predicted $m_{t}$ value may shift upwards by as much as a few $\mathrm{GeV}$ [62].

The near future promises a clarification of the value of the left-right asymmetry published by the SLD group and the ratio $R_{b}$ from LEP experiments. The precision scan around the $Z$ resonance performed 1993 by the four LEP experiments will further improve substantially the $Z$ resonance parameters. It would be advantageous to publish the data without the subtraction of the $Z-\gamma$ interference contribution. Eagerly awaited is the definitive observation of the top quark. If its mass turns out to be compatible with the electroweak analysis of the 1-loop effects there is hope to constrain for the first time the elusive Higgs sector.

By introducing the QCD coupling strength $\alpha_{s}\left(m_{Z}\right)_{\overline{\mathrm{MS}}}$ and the shift $\delta_{\alpha} \equiv 1 / \bar{\alpha}-128.72$ as external parameters in the fit, we have made clear the significance of their precise measurements. Unless these parameters are accurately measured, the search for effects beyond the SM through the electroweak radiative effects gets increasingly limited.

\section{Acknowledgements}

We thank K. Hara, H. Masuda and T. Mori for their help in understanding the experimental data. We also thank B.K. Bullock, S. Ishihara, B. Kniehl, K. Kondo, P. Langacker, J. Schneps, R. Szalapski, Y. Yamada and D. Zeppenfeld for clarifying discussions. The work of CSK was supported in part by the Korean Science and Engineering Foundation, in part by Non-Direct-Research-Fund, Korea Research Foundation 1993, and in part by the Basic Science Research Institute Program, Ministry of Education, 1994, Project No. BSRI-94-2425. 


\section{App. A SM radiative corrections at one-loop order}

In this appendix the propagator, vertex and box corrections of the standard model $(\mathrm{SM})$ are presented, all at one-loop level and partly at two-loop level for the $O\left(\alpha \alpha_{s}\right)$ terms of the gauge boson propagators. All the Green's functions are calculated in the 't Hooft Feynman gauge in the dimensional regularization and renormalized in the $\overline{\mathrm{MS}}$ scheme. Definitions of the scalar one-loop integrals, $A, B, C, D$ functions, are given in Appendix D. Vector boson propagators are given in A.1, the vector boson fermion vertex functions and the fermion wave function corrections follow in A.2, while the box corrections are listed in A.3. All the one-loop calculations are done independently and we reproduce the known results of ref. [2, 32, 113] for two-point functions and those of ref. [41 44] for the three- and four-point functions.

\section{A.1 Propagator corrections}

There are four vector boson two-point functions contributing to processes with external light quarks and leptons at one-loop order. They can be parametrized by [0]

$$
\begin{aligned}
\bar{\Pi}_{T}^{\gamma \gamma}\left(q^{2}\right) & =\hat{e}^{2} \bar{\Pi}_{T}^{Q Q}\left(q^{2}\right), \\
\bar{\Pi}_{T}^{Z \gamma}\left(q^{2}\right) & =\hat{e} \hat{g}_{Z}\left\{\bar{\Pi}_{T}^{3 Q}\left(q^{2}\right)-\hat{s}^{2} \bar{\Pi}_{T}^{Q Q}\left(q^{2}\right)\right\} \\
\bar{\Pi}_{T}^{Z Z}\left(q^{2}\right) & =\hat{g}_{Z}^{2}\left\{\bar{\Pi}_{T}^{33}\left(q^{2}\right)-2 \hat{s}^{2} \bar{\Pi}_{T}^{3 Q}\left(q^{2}\right)+\hat{s}^{4} \bar{\Pi}_{T}^{Q Q}\left(q^{2}\right)\right\} \\
\bar{\Pi}_{T}^{W W}\left(q^{2}\right) & =\hat{g}^{2} \bar{\Pi}_{T}^{11}\left(q^{2}\right),
\end{aligned}
$$

with the coupling factors

$$
\hat{g}_{Z}=\frac{\hat{g}}{\hat{c}}=\frac{\hat{e}}{\hat{s} \hat{c}},
$$

and the use of the compact notation

$$
\hat{s}^{2}=1-\hat{c}^{2}=\sin ^{2} \hat{\theta}_{W}
$$

throughout the appendix. These two-point functions and the coupling factors are renormalized in the $\overline{\mathrm{MS}}$ (the modified minimal subtraction) scheme, and hence they depend on the 't Hooft unit of mass $\mu$ which appears explicitly in the $B$ functions as defined in appendix D. The coupling factors of (A.2) and (A.3) also depend implicitly on the unit of mass $\mu$. The subscripts $T$ in eqs. (A.1) denote the transverse part of the polarization tensor

$$
\Pi_{\mu \nu}\left(q^{2}\right)=\left(-g_{\mu \nu}+\frac{q_{\mu} q_{\nu}}{q^{2}}\right) \Pi_{T}\left(q^{2}\right)+\frac{q_{\mu} q_{\nu}}{q^{2}} \Pi_{L}\left(q^{2}\right) .
$$

The longitudinal parts $\Pi_{L}\left(q^{2}\right)$ do not contribute to processes with light external fermions. 
With the help of the four $B$ functions, $B_{0}, B_{3}, B_{4}$ and $B_{5}$ (see appendix D), all SM contributions to the above two-point functions are expressed compactly. $\bar{\Pi}_{T}^{A B}\left(q^{2}\right)^{\prime}$ s is decomposed into the bosonic and the fermionic contributions,

$$
\bar{\Pi}_{T}^{A B}\left(q^{2}\right)=\bar{\Pi}_{T}^{A B}\left(q^{2}\right)_{B}+\Pi_{T}^{A B}\left(q^{2}\right)_{F},
$$

and the expressions are given separately.

\section{A.1.1 Bosonic contributions}

The bosonic contributions with pinch terms are given by [34]

$$
\begin{aligned}
\bar{\Pi}_{T}^{Q Q}\left(q^{2}\right)_{B} & =\Pi_{T}^{Q Q}\left(q^{2}\right)_{B}-\frac{1}{4 \pi^{2}} q^{2} B_{0}\left(q^{2} ; W, W\right), \\
\bar{\Pi}_{T}^{3 Q}\left(q^{2}\right)_{B} & =\Pi_{T}^{3 Q}\left(q^{2}\right)_{B}-\frac{1}{4 \pi^{2}}\left(q^{2}-\frac{1}{2} m_{W}^{2}\right) B_{0}\left(q^{2} ; W, W\right), \\
\bar{\Pi}_{T}^{33}\left(q^{2}\right)_{B} & =\Pi_{T}^{33}\left(q^{2}\right)_{B}-\frac{1}{4 \pi^{2}}\left(q^{2}-m_{W}^{2}\right) B_{0}\left(q^{2} ; W, W\right), \\
\bar{\Pi}_{T}^{11}\left(q^{2}\right)_{B} & =\Pi_{T}^{11}\left(q^{2}\right)_{B}-\frac{1}{4 \pi^{2}}\left(q^{2}-m_{W}^{2}\right)\left[\hat{c}^{2} B_{0}\left(q^{2} ; W, Z\right)+\hat{s}^{2} B_{0}\left(q^{2} ; W, \gamma\right)\right],
\end{aligned}
$$

where the short-hand notation

$$
B_{n}\left(q^{2} ; A, B\right)=B_{n}\left(q^{2} ; m_{A}, m_{B}\right),
$$

is introduced for the $B$ functions. Each $\Pi_{T}\left(q^{2}\right)$ function without overline is calculated in the 't Hooft-Feynman gauge, whereas the $\bar{\Pi}_{T}\left(q^{2}\right)$ functions with pinch terms are gauge invariant [34. The explicit expressions are

$$
\begin{aligned}
\bar{\Pi}_{T}^{Q Q}\left(q^{2}\right)_{B}= & -\frac{q^{2}}{16 \pi^{2}}\left\{\left[5 B_{0}+12 B_{3}\right]\left(q^{2} ; W, W\right)+\frac{2}{3}\right\} \\
\bar{\Pi}_{T}^{3 Q}\left(q^{2}\right)_{B}= & -\frac{q^{2}}{16 \pi^{2}}\left\{\left[\frac{11}{2} B_{0}+10 B_{3}\right]\left(q^{2} ; W, W\right)+\frac{2}{3}\right\} \\
\bar{\Pi}_{T}^{33}\left(q^{2}\right)_{B}= & \frac{1}{16 \pi^{2}}\left[m_{Z}^{2} B_{0}+\frac{1}{4} B_{5}\right]\left(q^{2} ; Z, H\right) \\
& -\frac{1}{16 \pi^{2}}\left[\left(\frac{23}{4} q^{2}-2 m_{W}^{2}\right) B_{0}+9 q^{2} B_{3}\right]\left(q^{2} ; W, W\right)-\frac{q^{2}}{24 \pi^{2}} \\
\bar{\Pi}_{T}^{11}\left(q^{2}\right)_{B}= & \frac{1}{16 \pi^{2}}\left[m_{W}^{2} B_{0}+\frac{1}{4} B_{5}\right](q ; W, H) \\
& -\frac{1}{16 \pi^{2}}\left[\left(8 \hat{c}^{2} q^{2}-\left(1-4 \hat{s}^{2}\right) m_{W}^{2}-m_{Z}^{2}\right) B_{0}-\left(\frac{9}{4}-2 \hat{s}^{2}\right) B_{5}\right]\left(q^{2} ; W, Z\right) \\
& -\frac{\hat{s}^{2}}{8 \pi^{2}}\left[\left(4 q^{2}-2 m_{W}^{2}\right) B_{0}-B_{5}\right]\left(q^{2} ; W, \gamma\right)-\frac{q^{2}}{24 \pi^{2}}
\end{aligned}
$$

At one-loop order of the minimal SM, the first terms in eqs. (A.8d) and (A.8d) are the only ones in the transverse component of the vector boson propagators being dependent on the Higgs boson mass $\left(m_{H}\right)$. 


\section{A.1.2 Fermionic contributions}

The fermionic contributions to the gauge boson propagators are known to $O\left(\alpha \alpha_{s}\right)$ two-loop level :

$$
\begin{aligned}
\Pi_{T}^{Q Q}\left(q^{2}\right)_{F}= & \frac{q^{2}}{16 \pi^{2}} \sum_{i} Q_{\ell}^{2} 8 B_{3}\left(q^{2} ; \ell_{i}, \ell_{i}\right) \\
& +\frac{q^{2}}{16 \pi^{2}} C_{q} \sum_{f=u_{i}, d_{i}} Q_{f}^{2}\left\{8 B_{3}\left(q^{2} ; f, f\right)+\frac{4}{3} \frac{\alpha_{s}}{\pi} B_{V}^{\prime}\left(q^{2} ; f, f\right)\right\}, \\
\Pi_{T}^{3 Q}\left(q^{2}\right)_{F}= & \frac{q^{2}}{16 \pi^{2}} \sum_{i} Q_{\ell} I_{3 \ell} 4 B_{3}\left(q^{2} ; \ell_{i}, \ell_{i}\right), \\
& +\frac{q^{2}}{16 \pi^{2}} C_{q} \sum_{f=u_{i}, d_{i}} Q_{f} I_{3 f}\left\{4 B_{3}\left(q^{2} ; f, f\right)+\frac{2}{3} \frac{\alpha_{s}}{\pi} B_{V}^{\prime}\left(q^{2} ; f, f\right)\right\}, \\
\Pi_{T}^{33}\left(q^{2}\right)_{F}= & \frac{1}{16 \pi^{2}} \sum_{f=\ell_{i}, \nu_{i}}\left(I_{3 f}\right)^{2}\left[4 q^{2} B_{3}-2 m_{f}^{2} B_{0}\right]\left(q^{2} ; f, f\right) \\
& +\frac{1}{16 \pi^{2}} C_{q} \sum_{f=u_{i}, d_{i}}\left(I_{3 f}\right)^{2}\left\{\left[4 q^{2} B_{3}-2 m_{f}^{2} B_{0}\right]\left(q^{2} ; f, f\right)+\frac{1}{3} \frac{\alpha_{s}}{\pi}\left[B_{V}+B_{A}\right]\left(q^{2} ; f, f\right)\right\}, \\
\Pi_{T}^{11}\left(q^{2}\right)_{F}= & \frac{1}{16 \pi^{2}} \sum_{i}\left[2 q^{2} B_{3}-B_{4}\right]\left(q^{2} ; \nu_{i}, \ell_{i}\right) \\
& +\frac{1}{16 \pi^{2}} C_{q} \sum_{i, j}\left|V_{u_{i} d_{j}}\right|^{2}\left\{\left[2 q^{2} B_{3}-B_{4}\right]\left(q^{2} ; u_{i}, d_{j}\right)+\frac{1}{6} \frac{\alpha_{s}}{\pi}\left[B_{V}+B_{A}\right]\left(q^{2} ; u_{i}, d_{j}\right)\right\},
\end{aligned}
$$

The summation over $i, j$ extends over the three generations of lepton and quark flavors, $\left(\nu_{1}, \nu_{2}, \nu_{3}\right)=\left(\nu_{e}, \nu_{\mu}, \nu_{\tau}\right),\left(\ell_{1}, \ell_{2}, \ell_{3}\right)=(e, \mu, \tau),\left(u_{1}, u_{2}, u_{3}\right)=(u, c, t)$ and $\left(d_{1}, d_{2}, d_{3}\right)=$ $(d, s, b) . C_{q}=3$ is the color factor, $Q_{f}$ the electric charge of the fermion $f$ in units of the proton charge, $I_{3 f}$ the weak isospin

$$
I_{3 f}=\left\{\begin{aligned}
+\frac{1}{2} & \text { for } f=\nu_{i} \text { or } u_{i L} \\
-\frac{1}{2} & \text { for } f=\ell_{i L} \text { or } d_{i L} \\
0 & \text { for } f=\ell_{i R}, u_{i R} \text { or } d_{i R}
\end{aligned}\right.
$$

while $V_{u_{i} d_{j}}$ are the Kobayashi-Maskawa quark mixing matrix elements. The $O\left(\alpha \alpha_{s}\right)$ corrections in perturbative QCD [52, 55, 56] are given by the functions $B_{V}$ and $B_{A}$ :

$$
\begin{aligned}
B_{V}\left(q^{2} ; m, m\right) & =q^{2} B_{V}^{\prime}\left(q^{2} ; m, m\right), \\
B_{A}\left(q^{2} ; m, m\right) & =q^{2} B_{A}^{\prime}\left(q^{2} ; m, m\right)+B_{A}(0 ; m, m), \\
B_{V}\left(q^{2} ; m, 0\right) & =q^{2} B_{V}^{\prime}\left(q^{2} ; m, 0\right)+B_{V}(0 ; m, 0), \\
B_{A}\left(q^{2} ; m, 0\right) & =q^{2} B_{A}^{\prime}\left(q^{2} ; m, 0\right)+B_{A}(0 ; m, 0),
\end{aligned}
$$


where

$$
\begin{aligned}
B_{V}^{\prime}\left(q^{2} ; m, m\right) & =\ln \frac{\mu^{2}}{m^{2}}+\frac{55}{12}-4 \zeta_{3}+\frac{4 m^{2}}{q^{2}} V_{1}\left(\frac{q^{2}}{4 m^{2}}\right) \\
B_{A}^{\prime}\left(q^{2} ; m, m\right) & =\ln \frac{\mu^{2}}{m^{2}}+\frac{55}{12}-4 \zeta_{3}+\frac{4 m^{2}}{q^{2}}\left[A_{1}\left(\frac{q^{2}}{4 m^{2}}\right)-A_{1}(0)\right], \\
B_{V}^{\prime}\left(q^{2} ; m, 0\right) & =B_{A}^{\prime}\left(q^{2} ; m, 0\right) \\
& =\ln \frac{\mu^{2}}{m^{2}}+\frac{55}{12}-4 \zeta_{3}+\frac{4 m^{2}}{q^{2}}\left[F_{1}\left(\frac{q^{2}}{m^{2}}\right)-F_{1}(0)\right]
\end{aligned}
$$

and

$$
\begin{aligned}
B_{A}(0 ; m, m) & =m^{2}\left[12 \ln ^{2} \frac{\mu^{2}}{m^{2}}+22 \ln \frac{\mu^{2}}{m^{2}}+\frac{31}{2}\right], \\
B_{V}(0 ; m, 0) & =B_{A}(0 ; m, 0) \\
& =m^{2}\left[3 \ln ^{2} \frac{\mu^{2}}{m^{2}}+\frac{11}{2} \ln \frac{\mu^{2}}{m^{2}}+\zeta_{2}+\frac{35}{8}\right] .
\end{aligned}
$$

Here $\zeta_{2}=\pi^{2} / 6, \zeta_{3}=1.2020569$, and the complex functions $V_{1}, A_{1}$ and $F_{1}$ are given in refs. [55, 56]. The following limits are useful :

$$
\begin{aligned}
B_{V}^{\prime}\left(M^{2} ; 0,0\right) & =B_{A}^{\prime}\left(M^{2} ; 0,0\right) \\
& =\ln \frac{\mu^{2}}{M^{2}}+\frac{55}{12}-4 \zeta_{3}+i \pi \\
B_{V}^{\prime}\left(M^{2} ; m, m\right) & =\ln \frac{\mu^{2}}{m^{2}}+\frac{15}{4}+O\left(\frac{M^{2}}{m^{2}}\right) \\
B_{A}^{\prime}\left(M^{2} ; m, m\right) & =\ln \frac{\mu^{2}}{m^{2}}+\frac{67}{36}+O\left(\frac{M^{2}}{m^{2}}\right), \\
B_{V}^{\prime}\left(M^{2} ; m, 0\right) & =B_{A}^{\prime}\left(M^{2} ; m, 0\right) \\
& =\ln \frac{\mu^{2}}{m^{2}}+\frac{115}{36}-\frac{4}{9} \zeta_{2}+O\left(\frac{M^{2}}{m^{2}}\right) .
\end{aligned}
$$

\section{A.2 Vertex correction}

The vertex form factors $\Gamma_{1}^{f}\left(q^{2}\right), \bar{\Gamma}_{2}^{f}\left(q^{2}\right), \Gamma_{3}^{f}\left(q^{2}\right)$ and $\Gamma_{4}^{f}\left(q^{2}\right)$ appearing in the helicity amplitudes (2.2) contribute to the $\gamma f f$ and $Z f f$ vertices as follows:

$$
\begin{aligned}
\Gamma^{\gamma f f}\left(q^{2}\right) & =-\hat{e}\left\{Q_{f}\left[1+\Gamma_{1}^{f}\left(q^{2}\right)\right]+I_{3 f} \bar{\Gamma}_{2}^{f}\left(q^{2}\right)\right\} \\
\Gamma^{Z f f}\left(q^{2}\right) & =-\hat{g}_{Z}\left\{\left(I_{3 f}-Q_{f} \hat{s}^{2}\right)\left[1+\Gamma_{1}^{f}\left(q^{2}\right)\right]+I_{3 f}\left[\hat{c}^{2} \bar{\Gamma}_{2}^{f}\left(q^{2}\right)+\Gamma_{3}^{f}\left(q^{2}\right)\right]+\Gamma_{4}^{f}\left(q^{2}\right)\right\} .
\end{aligned}
$$

It should be noted that the functions $\Gamma_{1}^{f}\left(q^{2}\right)$ and $\bar{\Gamma}_{2}^{f}\left(q^{2}\right)$ are common to the $\gamma f f$ and $Z f f$ vertices, and that $\Gamma_{3}^{f}\left(q^{2}\right)$ and $\Gamma_{4}^{f}\left(q^{2}\right)$ are additional contributions to the $Z f f$ vertex. These vertex functions depend on the chirality of $f$ and their explicit forms at one-loop 
level of the SM are

$$
\begin{aligned}
& \Gamma_{1}^{f_{R}}\left(q^{2}\right)=\left(\frac{g_{R}^{Z f f}}{4 \pi}\right)^{2} \Gamma_{1 Z}^{f}\left(q^{2}\right), \\
& \Gamma_{2}^{f_{R}}\left(q^{2}\right)=\Gamma_{3}^{f_{R}}\left(q^{2}\right)=\Gamma_{4}^{f_{R}}\left(q^{2}\right)=0, \\
& \Gamma_{1}^{f_{L}}\left(q^{2}\right)=\left(\frac{g_{L}^{Z f f}}{4 \pi}\right)^{2} \Gamma_{1 Z}^{f}\left(q^{2}\right)+\sum_{f^{\prime}}\left|\frac{g_{L}^{W f f^{\prime}}}{4 \pi}\right|^{2} \Gamma_{1 W}^{f^{\prime}}\left(q^{2}\right), \\
& \Gamma_{2}^{f_{L}}\left(q^{2}\right)=-2 \sum_{f^{\prime}}\left|\frac{g_{L}^{W f f^{\prime}}}{4 \pi}\right|^{2} \bar{\Gamma}_{2 W}^{f^{\prime}}\left(q^{2}\right), \\
& \Gamma_{3}^{f_{L}}\left(q^{2}\right)=\sum_{f^{\prime}}\left|\frac{g_{L}^{W f f^{\prime}}}{4 \pi}\right|^{2} \Gamma_{m W}^{f^{\prime}}\left(q^{2}\right), \\
& \Gamma_{4}^{f_{L}}\left(q^{2}\right)=0
\end{aligned}
$$

with the gauge boson coupling convention

$$
\begin{array}{cc}
g_{L}^{\gamma f f}=g_{R}^{\gamma f f}=\hat{e} Q_{f}, & g_{R}^{Z f f}=-\hat{g}_{Z} Q_{f} \hat{s}^{2}, \\
g_{L}^{Z f f}=\hat{g}_{Z}\left(I_{3 f}-Q_{f} \hat{s}^{2}\right), & g_{L}^{W f f^{\prime}}=\frac{\hat{g}}{\sqrt{2}} V_{f f^{\prime}},
\end{array}
$$

where

$$
\begin{aligned}
\Gamma_{1 Z}^{f}\left(q^{2}\right) & =\Gamma_{1}\left(q^{2} ; f, Z, f\right)-\Sigma^{\prime}\left(m_{f}^{2} ; f, Z\right) \\
\Gamma_{1 W}^{f^{\prime}}\left(q^{2}\right) & =\left(\Gamma_{1}+\Gamma_{1 m}\right)\left(q^{2} ; f^{\prime}, W, f^{\prime}\right)-\Sigma^{\prime}\left(m_{f}^{2} ; f^{\prime}, W\right) \\
\bar{\Gamma}_{2 W}^{f^{\prime}}\left(q^{2}\right) & =\left(\Gamma_{1}+\Gamma_{1 m}\right)\left(q^{2} ; f^{\prime}, W, f^{\prime}\right)-\Gamma_{2}\left(q^{2} ; W, f^{\prime}, W\right)+2 \operatorname{Re} B_{0}\left(q^{2} ; W, W\right) \\
\Gamma_{m W}^{f^{\prime}}\left(q^{2}\right) & =\Gamma_{1 m}\left(q^{2} ; f^{\prime}, W, f^{\prime}\right)+\Gamma_{2 m}\left(q^{2} ; W, f^{\prime}, W\right)
\end{aligned}
$$

Here

$$
\Sigma^{\prime}\left(q^{2} ; m, M\right)=-\left(2+\frac{m^{2}}{M^{2}}\right) B_{1}\left(q^{2} ; m, M\right)-1,
$$

is the external light fermion self energy correction, and the last term in eqs. (A.18G) of $\bar{\Gamma}_{2 W}^{f^{\prime}}$ is the pinch term [2, 34] which is subtracted from the vertex functions as calculated in the 't Hooft-Feynman gauge. The remaining vertex functions in eqs. (A.18) are

$$
\begin{aligned}
\Gamma_{1}\left(q^{2} ; m, M, m\right) & =\left[2 q^{2}\left(C_{11}+C_{23}\right)+4 C_{24}-\frac{m^{4}}{M^{2}} C_{0}\right]\left(q^{2} ; m, M, m\right)-2, \\
\Gamma_{1 m}\left(q^{2} ; m, M, m\right) & =\frac{m^{2}}{M^{2}}\left\{\left[q^{2}\left(C_{12}+C_{23}\right)+2 C_{24}-2 M^{2} C_{0}\right]\left(q^{2} ; m, M, m\right)-\frac{1}{2}\right\},(\text { (A.20b) } \\
\Gamma_{2}\left(q^{2} ; M, m, M\right) & =2\left[q^{2}\left(C_{11}+C_{23}\right)+\left(6+\frac{m^{2}}{M^{2}}\right) C_{24}+\left(q^{2}-m^{2}\right) C_{0}\right]\left(q^{2} ; M, m, M\right)-2, \\
\Gamma_{2 m}\left(q^{2} ; M, m, M\right) & =2 \frac{m^{2}}{M^{2}}\left[2 M^{2} C_{0}-C_{24}\right]\left(q^{2} ; M, m, M\right),
\end{aligned}
$$


with the shorthand notation for the $C$ functions of appendix D:

$$
C_{i}\left(q^{2} ; m_{1}, m_{2}, m_{3}\right) \equiv C_{i}\left(0,0, q^{2} ; m_{1}, m_{2}, m_{3}\right) .
$$

In the limit of the diagonal KM matrix elements $V_{u_{i} d_{j}}=\delta_{i j}$, which is assumed in all our numerical results, the internal fermion mass $m=m_{f^{\prime}}$ is non-negligible only for $f=b_{L}\left(f^{\prime}=t\right)$. Otherwise we can set $m=0$ at high energies $\left(m^{2} / q^{2} \sim 0\right)$ and find

$$
\begin{aligned}
\Gamma_{1}\left(q^{2} ; 0, M, 0\right)= & \ln \frac{\mu^{2}}{M^{2}}-4-2 \frac{M^{2}}{q^{2}}+\left(3+2 \frac{M^{2}}{q^{2}}\right) \ln \frac{-q^{2}-i \epsilon}{M^{2}} \\
& +2\left(1+\frac{M^{2}}{q^{2}}\right)^{2}\left[\operatorname{Sp}\left(1+\frac{q^{2}}{M^{2}}+i \epsilon\right)-\operatorname{Sp}(1)\right] \\
\Gamma_{2}\left(q^{2} ; M, 0, M\right)= & 3 \ln \frac{\mu^{2}}{M^{2}}+2-2 \frac{M^{2}}{q^{2}}+\left(1+2 \frac{M^{2}}{q^{2}}\right) \beta L+2 \frac{M^{2}}{q^{2}}\left(2+\frac{M^{2}}{q^{2}}\right) L^{2}, \\
\Gamma_{1 m}\left(q^{2} ; 0, M, 0\right)= & \Gamma_{2 m}\left(q^{2} ; M, 0, M\right)=0
\end{aligned}
$$

and

$$
\begin{aligned}
\Sigma^{\prime}(0 ; 0, M) & =\ln \frac{\mu^{2}}{M^{2}}-\frac{1}{2}, \\
\operatorname{Re} B_{0}\left(q^{2} ; M, M\right) & =\ln \frac{\mu^{2}}{M^{2}}+2-\beta L .
\end{aligned}
$$

Here $\operatorname{Sp}(z)=-\int_{0}^{z} \frac{1-t}{t} d t$ is the complex Spence (dilogarithm) function, and

$$
\begin{aligned}
& \beta=\sqrt{1-4\left(M^{2}-i \epsilon\right) / q^{2}}, \\
& L=\ln \frac{\beta+1}{\beta-1} .
\end{aligned}
$$

At low energies, light fermion masses may not be neglected as compared to the momentum transfer $q^{2}$. In the limit of $\left|q^{2}\right| / m_{Z}^{2} \ll 1$ and $m_{f}^{2} / m_{Z}^{2} \ll 1$, but at fixed $m_{f}^{2} / q^{2}$, the vertex functions reduce to

$$
\begin{aligned}
\Gamma_{1 Z}^{f}\left(q^{2}\right) & =\frac{-q^{2}}{m_{Z}^{2}}\left[J_{Z}\left(q^{2} ; f\right)+O\left(\frac{q^{2}}{m_{Z}^{2}}\right)\right], \\
\Gamma_{1 W}^{f^{\prime}}\left(q^{2}\right) & =\frac{-q^{2}}{m_{W}^{2}}\left[J_{W}\left(q^{2} ; f^{\prime}\right)+O\left(\frac{q^{2}}{m_{Z}^{2}}\right)\right], \\
\bar{\Gamma}_{2 W}^{f^{\prime}}\left(q^{2}\right) & =\frac{-q^{2}}{m_{W}^{2}}\left[\bar{J}_{W}\left(q^{2} ; f^{\prime}\right)+O\left(\frac{q^{2}}{m_{Z}^{2}}\right)\right],
\end{aligned}
$$

where

$$
\begin{aligned}
J_{V}\left(q^{2} ; f\right) & =4 F_{3}\left(q^{2} ; f, f\right)-\frac{2}{3} \ln m_{V}^{2}-\frac{1}{9}, \\
\bar{J}_{W}\left(q^{2} ; f\right) & =4 F_{3}\left(q^{2} ; f, f\right)-\frac{2}{3} \ln m_{W}^{2}-\frac{2}{3}-\frac{1}{3} .
\end{aligned}
$$

The function $F_{3}$ is defined in appendix D. The last $1 / 3$ factor is the pinch term. 


\section{A.3 Box correction}

Box corrections for the process $e_{\lambda} \overline{e_{\lambda}} \rightarrow f_{\sigma} \overline{f_{\sigma}}$ are expressed by $B_{\lambda \sigma}^{e f}$, where $\lambda, \sigma=-1$ is used for left-handed fermions and $\lambda, \sigma=+1$ for right-handed fermions.

$$
\begin{aligned}
& B_{\lambda, \lambda}^{e f}(s, t)=\frac{1}{16 \pi^{2}}\left|g_{\lambda}^{Z e e} g_{\lambda}^{Z f f}\right|^{2}\left[I_{1}\left(u, s ; m_{Z}, m_{f}\right)-I_{2}\left(t, s ; m_{Z}, m_{f}\right)\right] \\
& +\frac{\delta_{\lambda L}}{16 \pi^{2}}\left|g_{L}^{W e \nu} g_{L}^{W f f^{\prime}}\right|^{2} \times\left\{\begin{array}{ll}
+I_{1}\left(u, s ; m_{W}, m_{f^{\prime}}\right) & \text { for } I_{3 f}=+\frac{1}{2}\left(f=\nu_{\ell}, u_{i}\right) \\
-I_{2}\left(t, s ; m_{W}, m_{f^{\prime}}\right) & \text { for } I_{3 f}=-\frac{1}{2}\left(f=\ell, d_{i}\right)
\end{array},\right. \\
& B_{\lambda,-\lambda}^{e f}(s, t)=\frac{1}{16 \pi^{2}}\left|g_{\lambda}^{Z e e} g_{-\lambda}^{Z f f}\right|^{2}\left[I_{2}\left(u, s ; m_{Z}, m_{f}\right)-I_{1}\left(t, s ; m_{Z}, m_{f}\right)\right],
\end{aligned}
$$

with

$$
\begin{aligned}
s & =\left(p_{e}+p_{\bar{e}}\right)^{2}=\left(p_{f}+p_{\bar{f}}\right)^{2}, \\
t & =\left(p_{e}-p_{f}\right)^{2}=\left(p_{\bar{e}}-p_{\bar{f}}\right)^{2}, \\
u & =\left(p_{e}-p_{\bar{f}}\right)^{2}=\left(p_{\bar{e}}-p_{f}\right)^{2},
\end{aligned}
$$

and $p_{i}$ being the 4-momentum of particle $i$. The internal fermion mass $m_{f^{\prime}}$ is nonnegligible only for $f=b_{L}$, for which the top quark contributes in the limit of the diagonal KM matrix elements. The functions $I_{1}\left(u, s ; m_{V}, m_{f}\right)$ and $I_{2}\left(u, s ; m_{V}, m_{f}\right)$ are expressed in terms of the $D$ functions of appendix D:

$$
\begin{aligned}
& I_{1}\left(u, s ; m_{V}, m_{f}\right)=-2 u\left(D_{11}+D_{12}-D_{13}+2 D_{24}\right)-4 t D_{25}-4 s D_{26}-16 D_{27}, \\
& I_{2}\left(u, s ; m_{V}, m_{f}\right)=-2 u\left(D_{11}+D_{24}-D_{25}\right)-4 D_{27}
\end{aligned}
$$

where

$$
D_{i} \equiv D_{i}\left(0,0,0,0, u, s ; 0, m_{V}, m_{f}, m_{V}\right), \quad i=0,11-13,21-27 .
$$

After reduction of the higher $D$ functions

$$
\begin{aligned}
I_{1}(u, s ; M, m)= & -2 C_{0}^{(124)}-2 C_{0}^{(234)}+2\left(u-m^{2}\right) D_{0}^{(1234)} \\
I_{2}(u, s ; M, m)= & \frac{2}{s+u}\left[B_{0}^{(13)}-B_{0}^{(24)}\right] \\
& +\frac{u}{(s+u)^{2}}\left(s+2 u+2 M^{2}-m^{2}\right)\left[C_{0}^{(123)}+C_{0}^{(134)}\right] \\
& -\frac{1}{(s+u)^{2}}\left(s\left(s+2 u-2 M^{2}+m^{2}\right)+2 u^{2}\right)\left[C_{0}^{(124)}+C_{0}^{(234)}\right] \\
& +\frac{1}{(s+u)^{2}}\left\{\left(u-m^{2}\right) s^{2}-\left(2 M^{4}-2 M^{2} m^{2}+m^{4}+m^{2} u-2 u^{2}\right) s\right. \\
& \left.+2\left(M^{2}+u\right)\left(M^{2}-m^{2}+u\right) u\right\} D_{0}^{(1234)}
\end{aligned}
$$


is obtained. For the case $f \neq b_{L}$ the limit $m \rightarrow 0$ can be carried out :

$$
\begin{aligned}
I_{1}(u, s ; M, 0)= & -4 C_{0}^{(124)}+2 u D_{0}^{(1234)} \\
I_{2}(u, s ; M, 0)= & \frac{2}{s+u}\left[B_{0}^{(13)}-B_{0}^{(24)}\right] \\
& +\frac{2}{(s+u)^{2}} u\left(s+2 u+2 M^{2}\right) C_{0}^{(123)} \\
& -\frac{2}{(s+u)^{2}}\left(s\left(s+2 u-2 M^{2}\right)+2 u^{2}\right) C_{0}^{(124)} \\
& +\frac{1}{(s+u)^{2}}\left\{s^{2} u-2 s\left(M^{4}-u^{2}\right)+2 u\left(M^{2}+u\right)^{2}\right\} D_{0}^{(1234)} .
\end{aligned}
$$

Eqs. A.33) agree exactly with ref. 41.

In the low energy limit, only $D_{27}$ survives:

$$
\begin{aligned}
D_{27}\left(0,0,0,0,0,0 ; 0, m_{V}, 0, m_{V}\right) & =\frac{1}{4 m_{V}^{2}} \\
D_{27}\left(0,0,0,0,0,0 ; 0, m_{W}, m_{t}, m_{W}\right) & =\frac{1}{4\left(m_{t}^{2}-m_{W}^{2}\right)}\left[\frac{m_{t}^{2}}{m_{t}^{2}-m_{W}^{2}} \ln \frac{m_{t}^{2}}{m_{W}^{2}}-1\right]
\end{aligned}
$$

and hence

$$
\begin{aligned}
B_{\lambda, \lambda}^{e f}(0,0) & =\frac{1}{16 \pi^{2}}\left|g_{\lambda}^{Z e e} g_{\lambda}^{Z f f}\right|^{2} \frac{3}{m_{Z}^{2}} \\
& +\frac{\delta_{\lambda L}}{16 \pi^{2}}\left|g_{L}^{W e \nu} g_{L}^{W f f^{\prime}}\right|^{2} \times\left\{\begin{array}{l}
+\frac{4}{m_{W}^{2}} \quad \text { for } I_{3 f}=+\frac{1}{2}\left(f=\nu_{\ell}, u_{i}\right) \\
-\frac{1}{m_{W}^{2}} \quad \text { for } I_{3 f}=-\frac{1}{2}\left(f=\ell, d_{i}\right)
\end{array},\right. \\
B_{\lambda,-\lambda}^{e f}(0,0) & =-\frac{1}{16 \pi^{2}}\left|g_{\lambda}^{Z e e} g_{-\lambda}^{Z f f}\right|^{2} \frac{3}{m_{Z}^{2}},
\end{aligned}
$$

for $f \neq b_{L}$, and

$$
B_{L, L}^{e b}(0,0)=\frac{\left|g_{L}^{Z e e} g_{L}^{Z b b}\right|^{2}}{16 \pi^{2}} \frac{3}{m_{Z}^{2}}-\frac{\left|g_{L}^{W e \nu} g_{L}^{W b t}\right|^{2}}{16 \pi^{2}} \frac{1}{m_{t}^{2}-m_{W}^{2}}\left[\frac{m_{t}^{2}}{m_{t}^{2}-m_{W}^{2}} \ln \frac{m_{t}^{2}}{m_{W}^{2}}-1\right]
$$

for $f=b_{L}$. 


\section{App. B Renormalization group and hadronic contributions}

The effective charges of the $S U(2) \times U(1)$ theory are expressed in terms of the $\overline{\mathrm{MS}}$ couplings by

$$
\begin{aligned}
& \frac{1}{\bar{e}^{2}\left(q^{2}\right)}=\frac{1}{\hat{e}^{2}(\mu)}+\operatorname{Re} \bar{\Pi}_{T, \gamma}^{Q Q}\left(q^{2}\right), \\
& \frac{1}{\bar{g}^{2}\left(q^{2}\right)}=\frac{1}{\hat{g}^{2}(\mu)}+\operatorname{Re} \bar{\Pi}_{T, \gamma}^{3 Q}\left(q^{2}\right),
\end{aligned}
$$

where the $S U(2)$ effective charge

$$
\bar{g}^{2}\left(q^{2}\right) \equiv \frac{\bar{e}^{2}\left(q^{2}\right)}{\bar{s}^{2}\left(q^{2}\right)}
$$

is introduced for convenience. The expressions (B.1) and (B.2) are explicit solutions of the renormalization group $(\mathrm{RG})$ equation in the $\overline{\mathrm{MS}}$ scheme :

$$
D[\text { effective charges }]=0,
$$

with the RG operator

$$
\begin{aligned}
D & \equiv\left[\mu^{2} \frac{d^{2}}{d \mu^{2}}\right]_{\text {Bare }} \\
& =\mu^{2} \frac{\partial}{\partial \mu^{2}}+\hat{\beta}_{e}\left(\frac{\hat{e}^{2}}{16 \pi^{2}}\right) \frac{\partial}{\partial\left(\hat{e}^{2} / 16 \pi^{2}\right)}+\hat{\beta}_{g}\left(\frac{\hat{g}^{2}}{16 \pi^{2}}\right) \frac{\partial}{\partial\left(\hat{g}^{2} / 16 \pi^{2}\right)} .
\end{aligned}
$$

The $\overline{\mathrm{MS}} \hat{\beta}$-functions in the minimal SM read at one-loop order

$$
\begin{aligned}
& \hat{\beta}_{e}\left(\frac{\hat{e}^{2}}{16 \pi^{2}}\right)=\left[-7+\frac{4}{3} \Sigma_{f} C_{f} Q_{f}^{2}\right]\left(\frac{\hat{e}^{2}}{16 \pi^{2}}\right)^{2} \\
& \hat{\beta}_{g}\left(\frac{\hat{g}^{2}}{16 \pi^{2}}\right)=\left[-\frac{43}{6}+\frac{2}{3} \Sigma_{f} C_{f} I_{3 f} Q_{f}\right]\left(\frac{\hat{g}^{2}}{16 \pi^{2}}\right)^{2},
\end{aligned}
$$

where $C_{f}=1(3)$ for $f=\ell(q)$. The two-loop $O\left(\alpha \alpha_{s}\right)$ contributions are accounted for by replacing $C_{q} \rightarrow C_{q}\left(1+\frac{\hat{\alpha}_{s}}{\pi}\right)$ in eqs. (B.6) and (B.7). Note that the effective charges $\bar{e}^{2}\left(q^{2}\right)$ and $\bar{g}^{2}\left(q^{2}\right)$ behaves similarly to the $\overline{\mathrm{MS}}$ couplings at asymptotically high energies, $\left|q^{2}\right| \gg m_{W}^{2}$, since the functions $\bar{\Pi}_{T, \gamma}^{Q Q}\left(q^{2}\right)$ and $\bar{\Pi}_{T, \gamma}^{3 Q}\left(q^{2}\right)$ do not have large logarithms at $\mu^{2} \sim\left|q^{2}\right| \gg m_{W}^{2}$ [2]. This is enabled by adding the pinch terms [2, 34] in the self energy $\bar{\Pi}\left(q^{2}\right)$, and our $\bar{e}^{2}\left(q^{2}\right)$ and $\bar{s}^{2}\left(q^{2}\right)$ are equivalent to the corresponding *-charges [2] up to the imaginary parts and the two-loop corrections.

Although the $\overline{\mathrm{MS}}$ couplings $\hat{e}$ and $\hat{g}$ could be adopted directly in our analysis, we prefer the effective charges of (B.1) and (B.2) as quantities to be used when confronting theory with experiment. We give two reasons, one being associated with the non-decoupling of 
heavy particles in the $\overline{\mathrm{MS}}$ scheme, the other being related with the treatment of nonperturbative hadronic contributions to the electroweak parameters.

Traditionally, the appearance of large logarithms of heavy particle masses (nondecoupling) in the $\overline{\mathrm{MS}}$ scheme is avoided by adopting the effective field theory [114, 115], where the heavy particle fields are integrated out in the action. The couplings of the effective theories are then related to each others by matching conditions ensuring that all effective theories give identical results at zero momentum transfer, since the effects of heavy particles in the effective light field theory must be proportional to $q^{2} / m_{\text {heavy }}^{2}$.

In general, the two $\overline{\mathrm{MS}}$ couplings $\hat{e}^{2}(\mu)_{\text {eff }}$ and $\hat{g}^{2}(\mu)_{\text {eff }}$ of the effective light particle theory can be obtained by the matching conditions

$$
\begin{aligned}
\frac{1}{\bar{e}^{2}(0)} & =\frac{1}{\hat{e}^{2}(\mu)_{e f f}}+\left[\operatorname{Re} \bar{\Pi}_{T, \gamma}^{Q Q}(0)\right]_{e f f}, \\
\frac{1}{\bar{g}^{2}(0)} & =\frac{1}{\hat{g}^{2}(\mu)_{e f f}}+\left[\operatorname{Re} \bar{\Pi}_{T, \gamma}^{3 Q}(0)\right]_{e f f},
\end{aligned}
$$

where only the light particles at the scale $\mu$ contribute to the two-point functions at the right-hand side. In the minimal SM, one may, for instance, employ an effective theory of particles of mass up to the scale $\mu$ :

$$
\begin{aligned}
& \frac{16 \pi^{2}}{\bar{e}^{2}(0)}=\frac{16 \pi^{2}}{\hat{e}^{2}(\mu)_{e f f}}+\frac{4}{3} \Sigma_{f} Q_{f}^{2} \ln \frac{\mu^{2}}{m_{f}^{2}} \theta\left(\mu-m_{f}\right)-\left(7 \ln \frac{\mu^{2}}{m_{W}^{2}}+\frac{2}{3}\right) \theta\left(\mu-m_{W}\right), \\
& \frac{16 \pi^{2}}{\bar{g}^{2}(0)}=\frac{16 \pi^{2}}{\hat{g}^{2}(\mu)_{e f f}}+\frac{2}{3} \Sigma_{f} I_{3 f} Q_{f} \ln \frac{\mu^{2}}{m_{f}^{2}} \theta\left(\mu-m_{f}\right)-\left(\frac{43}{6} \ln \frac{\mu^{2}}{m_{W}^{2}}+\frac{2}{3}\right) \theta\left(\mu-m_{W}\right),
\end{aligned}
$$

Such a scheme is often adopted in quantum chromodynamics (QCD), but leads to a discontinuity at $\mu=m_{W}$ of the effective $\overline{\mathrm{MS}}$ coupling constants. The appearance of the discontinuity in the unphysical $\overline{\mathrm{MS}}$ couplings is not really a problem】, but the appearance of many quark and lepton mass scales renders the use of these effective couplings impractical at the scale $\mu<m_{Z}$. Furthermore, direct use of the effective $\overline{\mathrm{MS}}$ couplings at lower energies leads to expressions with light-quark masses suffering from large non-perturbative QCD corrections.

These two problems of the $\overline{\mathrm{MS}}$ scheme can be overcome simultaneously by adopting the effective charges $(\mathrm{B} .1)$ and $(\mathrm{B} .2)$ as expansion parameters at

$$
0 \leq\left|q^{2}\right|<O\left(m_{Z}^{2}\right)
$$

when confronting with experiments. The connection with a high energy theory, e.g. at $q^{2}=m_{Z}^{2}$, can then be made free from light quark mass ambiguities by the use of the

${ }^{7}$ In fact the discontinuity can be evaded by using yet another unphysical effective coupling, the so called dimensional reduction $\overline{\mathrm{DR}}$ scheme 116 . 
manifestly RG invariant expressions (B.1) and (B.2). In the region (B.12) the effective charges at two different $q^{2}$ are related by dispersion relations.

The light hadron (first 5-quark, or " $5 q$ ") contributions to the differences

$$
\begin{aligned}
& \frac{1}{\bar{\alpha}\left(q^{2}\right)}-\frac{1}{\alpha}=\frac{4 \pi}{\bar{e}^{2}\left(q^{2}\right)}-\frac{4 \pi}{\bar{e}^{2}(0)}=4 \pi\left[\operatorname{Re} \bar{\Pi}_{T, \gamma}^{Q Q}\left(q^{2}\right)-\bar{\Pi}_{T, \gamma}^{Q Q}(0)\right], \\
& \frac{\bar{s}^{2}\left(q^{2}\right)}{\bar{\alpha}\left(q^{2}\right)}-\frac{\bar{s}^{2}(0)}{\alpha}=\frac{4 \pi}{\bar{g}^{2}\left(q^{2}\right)}-\frac{4 \pi}{\bar{g}^{2}(0)}=4 \pi\left[\operatorname{Re} \bar{\Pi}_{T, \gamma}^{3 Q}\left(q^{2}\right)-\bar{\Pi}_{T, \gamma}^{3 Q}(0)\right],
\end{aligned}
$$

have been parametrized in the region $0<\left|q^{2}\right|<m_{Z}^{2}$ as follows. For the photon vacuum polarization function, we use

$$
\begin{aligned}
4 \pi & {\left[\operatorname{Re} \bar{\Pi}_{T, \gamma}^{Q Q}\left(q^{2}\right)-\bar{\Pi}_{T, \gamma}^{Q Q}(0)\right]_{5 q} } \\
& =\left\{\begin{array}{lll}
-f\left(q^{2}\right) & \text { for } & -m_{Z}^{2}<q^{2}<0, \\
-f\left(q^{2}\right)+4 \pi \sum_{q=c, b}\left[\operatorname{Re} \bar{\Pi}_{T, \gamma}^{Q Q}\left(q^{2}\right)-\bar{\Pi}_{T, \gamma}^{Q Q}\left(-q^{2}\right)\right]_{q} & \text { for } & 0<q^{2}<m_{Z}^{2} .
\end{array}\right.
\end{aligned}
$$

Here the results of the dispersion integral analyses [27, 28] are parametrized by

$$
f\left(q^{2}\right)= \begin{cases}1.096 \ln \left(1+\left|q^{2}\right|\right) & \text { for } 0.0 \leq \sqrt{\left|q^{2}\right|}(\mathrm{GeV}) \leq 0.3 \\ 0.3261 \ln \left(1+3.927\left|q^{2}\right|\right) & \text { for } 0.3 \leq \sqrt{\left|q^{2}\right|}(\mathrm{GeV}) \leq 3 \\ 0.2486+0.4009 \ln \left(1+\left|q^{2}\right|\right) & \text { for } 3.0 \leq \sqrt{\left|q^{2}\right|}(\mathrm{GeV}) \leq 50 \\ 3.878+0.4084\left\{\ln \frac{\left|q^{2}\right|}{s_{0}}+0.005696\left(\frac{\left|q^{2}\right|}{s_{0}}-1\right)\right\} & \text { for } 50 \leq \sqrt{\left|q^{2}\right|}(\mathrm{GeV}) \leq m_{Z}\end{cases}
$$

with $s_{0}=(91.176 \mathrm{GeV})^{2}$. The parametrization (B.16) is copied from ref. [27 for $0 \mathrm{GeV}<$ $\sqrt{\left|q^{2}\right|}<50 \mathrm{GeV}$ and smoothly connected to the most recent estimates of ref. [28] at $q^{2}=m_{Z}^{2} ; f\left(m_{Z}^{2}\right)=(0.0283 \pm 0.0007) / \alpha$. In the time-like region $\left(0<q^{2}<m_{Z}^{2}\right)$, the second term in (B.15b) is added in order to account approximately for the threshold contributions of the charm and bottom quarks.

Hadronic contribution to the photon- $Z$ mixing two-point function can then be estimated as [29]

$$
4 \pi\left[\operatorname{Re} \bar{\Pi}_{T, \gamma}^{3 Q}\left(q^{2}\right)-\bar{\Pi}_{T, \gamma}^{3 Q}(0)\right]_{5 q}=2 \pi\left[\operatorname{Re} \bar{\Pi}_{T, \gamma}^{Q Q}\left(q^{2}\right)-\bar{\Pi}_{T, \gamma}^{Q Q}(0)\right]_{5 q}+\Delta_{\omega \phi}\left(q^{2}\right)+\Delta_{c}\left(q^{2}\right)+\Delta_{b}\left(q^{2}\right),
$$

where

$$
\Delta_{\omega \phi}\left(q^{2}\right)=\frac{3 q^{2}}{4 \alpha^{2}}\left\{\frac{2 \Gamma\left(\omega \rightarrow e^{+} e^{-}\right)}{m_{\omega}\left(m_{\omega}^{2}+\left|q^{2}\right|\right)}-\frac{\Gamma\left(\phi \rightarrow e^{+} e^{-}\right)}{m_{\phi}\left(m_{\phi}^{2}+\left|q^{2}\right|\right)}\right\}
$$


is an estimate [29] for the extra contribution from the $u, d, s$ quarks, and

$$
\begin{aligned}
\Delta_{q}\left(q^{2}\right)=\frac{1}{6 \pi} C_{q} Q_{q}\{ & B_{3}\left(0 ; m_{q}, m_{q}\right)-B_{3}\left(q^{2} ; m_{q}, m_{q}\right) \\
& \left.+\frac{\alpha_{s}}{6 \pi}\left[B_{V}^{\prime}\left(0 ; m_{q}, m_{q}\right)-B_{V}^{\prime}\left(q^{2} ; m_{q}, m_{q}\right)\right]\right\},
\end{aligned}
$$

for $q=c$ and $b$, are calculated perturbatively. Note that in the $m_{u}=m_{d}=m_{s}$ limit, the identity $\Delta_{u}+\Delta_{d}+\Delta_{s}=0$ holds. Thus, the term $\Delta_{\omega \phi}$ gives an estimate [29] of the flavor $\mathrm{SU}(3)$ violation effect. Contributions of leptons, the top quark and any other new particles, as well as the light 5-quark contributions at $\sqrt{\left|q^{2}\right|}>m_{Z}$ are treated perturbatively.

The light quark masses to be used in the region $\left|q^{2}\right|>m_{Z}^{2}$ are determined by requiring continuity of the two effective charges at $q^{2}=m_{Z}^{2}$. The left-hand sides of eqs. (B.15) and (B.17) are evaluated perturbatively, and equated with the estimate at $q^{2}=m_{Z}^{2}$ :

$$
\begin{aligned}
4 \pi\left[\operatorname{Re} \bar{\Pi}_{T, \gamma}^{Q Q}\left(m_{Z}^{2}\right)-\bar{\Pi}_{T, \gamma}^{Q Q}(0)\right]_{5 q} & =-f\left(m_{Z}^{2}\right), \\
4 \pi\left[\operatorname{Re} \bar{\Pi}_{T, \gamma}^{3 Q}\left(m_{Z}^{2}\right)-\bar{\Pi}_{T, \gamma}^{3 Q}(0)\right]_{5 q} & =-\frac{1}{2} f\left(m_{Z}^{2}\right)+\Delta_{\omega \phi}\left(m_{Z}^{2}\right)+\Delta_{c}\left(m_{Z}^{2}\right)+\Delta_{b}\left(m_{Z}^{2}\right),
\end{aligned}
$$

where the mean value of the estimate [28]

$$
-f\left(m_{Z}^{2}\right)=\frac{-0.0283 \pm 0.0007}{\alpha} \equiv-3.88+\delta_{\text {had }} ; \quad \delta_{\text {had }}=0 \pm 0.1
$$

is taken at $m_{Z}=91.187 \mathrm{GeV}$. Note that the additional term at the right-hand side of (B.15b) is less than 0.001 and the discontinuity at $q^{2}=m_{Z}^{2}$ is negligibly small. With the use of the expressions (A.9a) and (A.9b), the two matching conditions can be approximated by:

$$
\begin{aligned}
& \sum_{5 q} Q_{q}^{2}\left[\frac{1}{6} \ln \frac{m_{Z}^{2}}{m_{q}^{2}}-\frac{5}{18}\right]+O\left(\frac{m_{q}^{2}}{m_{Z}^{2}}, \frac{\alpha_{s}}{\pi}\right)=\frac{\pi}{6} f\left(m_{Z}^{2}\right), \\
& \ln \frac{m_{d} m_{s}}{m_{u}^{2}}+O\left(\frac{\alpha_{s}}{\pi}, \frac{m_{s}^{2}}{m_{Z}^{2}}\right)=18 \pi \Delta_{\omega \phi}\left(m_{Z}^{2}\right)=0.152 .
\end{aligned}
$$

Taking the charm and bottom quark masses

$$
\begin{aligned}
& m_{c}=1.4 \mathrm{GeV}, \\
& m_{b}=4.7 \mathrm{GeV},
\end{aligned}
$$

and including $O\left(\alpha_{s}\right)$ corrections one finds for $\delta_{\text {had }}=0$ :

\begin{tabular}{|c|c|c|c|c|}
\hline$\alpha_{s}\left(m_{Z}\right)$ & 0 & 0.11 & 0.12 & 0.13 \\
\hline$m_{u}=m_{d}(\mathrm{GeV})$ & 0.055 & 0.089 & 0.093 & 0.097 \\
\hline$m_{s}(\mathrm{GeV})$ & 0.064 & 0.104 & 0.108 & 0.113 \\
\hline
\end{tabular}


Our program finds appropriate light quark masses for arbitrary $\alpha_{s}\left(m_{Z}\right), \delta_{\text {had }}, m_{c}$ and $m_{b}$ input values by solving the continuity conditions (B.20) and (B.21). It should be pointed out here that these light quark masses are fixed merely to ensure the continuity of the effective charges at $q^{2}=m_{Z}^{2}$ and that they do not have a direct physical significance. At $\left|q^{2}\right|>m_{Z}^{2}$, where those quark masses are used, the mass effects are suppressed by $m_{q}^{2} / m_{Z}^{2}$ and never become significant. Whenever the light quark mass values play a physically significant role, their values must be chosen independent of those of eq. (B.26) by appropriate physics arguments.

In Fig. 2 the SM predictions for the effective charge $4 \pi / \bar{e}^{2}\left(q^{2}\right)$ and the effective weak mixing angle $\bar{s}^{2}\left(q^{2}\right)$ are shown in the region $1 \mathrm{MeV}<\sqrt{\left|q^{2}\right|}<1 \mathrm{TeV}$ for $m_{t}=100,150$, $200 \mathrm{GeV}$ and $m_{H}=100,1000 \mathrm{GeV}$ with $\delta_{\text {had }}=0$. The solid lines show the space-like $\left(q^{2}<0\right)$ effective charge, whereas the dashed lines the time-like $\left(q^{2}>0\right)$ effective charge. The top-quark effect at $q^{2}=m_{Z}^{2}$ can be parametrized by

$$
\begin{aligned}
\left.\frac{1}{\bar{\alpha}\left(m_{Z}^{2}\right)}\right)_{\mathrm{SM}} & =128.71+\delta_{\mathrm{had}}+0.024\left(1+5 \frac{\alpha_{s}}{\pi}\right)\left(\frac{100 \mathrm{GeV}}{m_{t}}\right)^{2}, \\
{\left[\frac{\bar{s}^{2}\left(m_{Z}^{2}\right)}{\bar{\alpha}\left(m_{Z}^{2}\right)}-\frac{\bar{s}^{2}(0)}{\alpha}\right]_{\mathrm{SM}} } & =-3.09+\frac{\delta_{\mathrm{had}}}{2}+0.009\left(1+5 \frac{\alpha_{s}}{\pi}\right)\left(\frac{100 \mathrm{GeV}}{m_{t}}\right)^{2},
\end{aligned}
$$

for $m_{t}>100 \mathrm{GeV}$ representing typical contributions of a heavy particle to the running of the effective charge form factor $\bar{\alpha}\left(q^{2}\right)$ and $\bar{s}^{2}\left(q^{2}\right)$ between $q^{2}=0$ and $m_{Z}^{2}$.

When constraining new physics contributions the value of $\bar{\alpha}\left(m_{Z}^{2}\right)$ is required, but only $\alpha=\bar{\alpha}(0)$, that is, the fine structure constant, is precisely measured. When new physics is contributing significantly to the running of the effective charge form factors between $q^{2}=0$ and $q^{2}=m_{Z}^{2}$, its value can deviate from the SM prediction (B.27). In order to account for both such new physics contributions and future improvements in the measurement of $\delta_{\text {had }}$, the parameter

$$
\delta_{\alpha} \equiv \frac{1}{\bar{\alpha}\left(m_{Z}^{2}\right)}-128.72 .
$$

is introduced. For instance, in the SM one finds from (B.27)

$$
\left[\delta_{\alpha}\right]_{\mathrm{SM}}=\delta_{\mathrm{had}}+0.024\left(1+5 \frac{\alpha_{s}}{\pi}\right)\left(\frac{100 \mathrm{GeV}}{m_{t}}\right)^{2}-0.01 .
$$

The last two terms are close to zero for $m_{t}=150-200 \mathrm{GeV}$, such that within the SM :

$$
\left[\delta_{\alpha}\right]_{\mathrm{SM}} \approx \delta_{\text {had }} .
$$

In general, new physics contributions can be accounted for by

$$
\delta_{\alpha}=\left[\delta_{\alpha}\right]_{\mathrm{SM}}+4 \pi\left[\operatorname{Re} \Pi_{T, \gamma}^{Q Q}\left(m_{Z}^{2}\right)-\Pi_{T, \gamma}^{Q Q}(0)\right]_{\mathrm{NewPhysics}} .
$$


An example of the extra term is found in ref. [11], where consequences of the gaugeinvariant dimension six operators [10] have been studied in detail.

The $\overline{\mathrm{MS}}$ couping constants $\hat{e}^{2}(\mu)$ and $\hat{g}^{2}(\mu)$ are determined from the identities eqs. (B.1) and (B.2) evaluated at large $\left|q^{2}\right|$, say at $q^{2}=m_{Z}^{2}$. The magnitude of $\hat{e}^{2}\left(m_{Z}\right)$ depends on $m_{t}$ and the assumed $\alpha_{s}\left(m_{Z}\right)$ value, and that of $\hat{s}^{2}\left(m_{Z}\right)$ depends also on the $\bar{s}^{2}\left(m_{Z}^{2}\right)$ value as observed at LEP/SLC. For $\alpha_{s}=0.12$ one obtains

$$
\frac{1}{\hat{\alpha}\left(m_{Z}\right)_{\mathrm{SM}}} \equiv \frac{4 \pi}{\hat{e}^{2}\left(m_{Z}\right)_{\mathrm{SM}}}=128.00+\delta_{\mathrm{had}}+ \begin{cases}-0.12 & \text { for } m_{t}=100 \mathrm{GeV} \\ +0.00 & \text { for } m_{t}=150 \mathrm{GeV} \\ +0.08 & \text { for } m_{t}=200 \mathrm{GeV} \\ +0.15 & \text { for } m_{t}=250 \mathrm{GeV}\end{cases}
$$

and

$$
\hat{s}^{2}\left(m_{Z}\right)_{\mathrm{SM}}=\bar{s}^{2}\left(m_{Z}^{2}\right)+\frac{\alpha}{2} \delta_{\mathrm{had}}+\left\{\begin{array}{ll}
0.0007 & \text { for } m_{t}=100 \mathrm{GeV} \\
0.0009 & \text { for } m_{t}=150 \mathrm{GeV} \\
0.0010 & \text { for } m_{t}=200 \mathrm{GeV} \\
0.0011 & \text { for } m_{t}=250 \mathrm{GeV}
\end{array},\right.
$$

The relatively large $m_{t}$ dependences above, as opposed to those of eqs. (B.27) and (B.28), result from the non-decoupling of the heavy top quark due to the logarithmic $m_{t}$ dependence of the $\overline{\mathrm{MS}}$ renormalized two-point functions in eqs. (B.1) and (B.2), as explained earlier. In the presence of many new particles at the $\mathrm{TeV}$ scale, such as in the supersymmetric standard model, all new particle contributions are suppressed by their inverse mass-squared as demonstrated for a heavy top quark in (B.27) and (B.28) for the effective charges, while the magnitude of $\hat{e}^{2}\left(m_{Z}\right)$ and $\hat{s}^{2}\left(m_{Z}\right)$ are affected strongly. One should then either adopt the effective light particle theory for the $\overline{\mathrm{MS}}$ couplings [5, 9, [7, 23] or use the above effective charges below $\mathrm{TeV}$ scale.

Finally, it is worth mentioning that the expressions for the running of the remaining two charge form factors:

$$
\begin{aligned}
& \frac{1}{\bar{g}_{Z}^{2}\left(q^{2}\right)}=\frac{1}{\hat{g}_{Z}^{2}(\mu)}+\operatorname{Re} \bar{\Pi}_{T, Z}^{33}\left(q^{2}\right)-2 \hat{s}^{2} \operatorname{Re} \bar{\Pi}_{T, Z}^{3 Q}\left(q^{2}\right)+\hat{s}^{4} \operatorname{Re} \bar{\Pi}_{T, Z}^{Q Q}\left(q^{2}\right), \\
& \frac{1}{\bar{g}_{W}^{2}\left(q^{2}\right)}=\frac{1}{\hat{g}^{2}(\mu)}+\operatorname{Re} \bar{\Pi}_{T, W}^{11}\left(q^{2}\right),
\end{aligned}
$$

are not the exact solution of the one-loop RG equations of the $\overline{\mathrm{MS}}$ couplings, but that the $O(1)$ terms at the right-hand sides remain small at all $q^{2}$, provided the renormalization condition

$$
\begin{aligned}
& \hat{e}^{2}(\mu)=\bar{e}^{2}\left(Q^{2}\right), \\
& \hat{s}^{2}(\mu)=\bar{s}^{2}\left(Q^{2}\right),
\end{aligned}
$$


is chosen with

$$
Q^{2}=\left\{\begin{array}{lll}
m_{Z}^{2} & \text { if } & \left|q^{2}\right| \lesssim O\left(m_{Z}^{2}\right), \\
q^{2} & \text { if } & \left|q^{2}\right|>m_{Z}^{2}
\end{array}\right.
$$

Therefore, $\bar{g}_{Z}^{2}\left(q^{2}\right)$ and $\bar{g}_{W}^{2}\left(q^{2}\right)$ can be safely calculated from

$$
\begin{aligned}
\frac{4 \pi}{\bar{g}_{Z}^{2}\left(q^{2}\right)} & =\frac{\bar{s}^{2}\left(Q^{2}\right) \bar{c}^{2}\left(Q^{2}\right)}{\bar{\alpha}\left(Q^{2}\right)}+\frac{1}{4} S_{Z}\left(q^{2}\right), \\
\frac{4 \pi}{\bar{g}_{W}^{2}\left(q^{2}\right)} & =\frac{\bar{s}^{2}\left(Q^{2}\right)}{\bar{\alpha}\left(Q^{2}\right)}+\frac{1}{4} S_{W}\left(q^{2}\right),
\end{aligned}
$$

where the two quantities

$$
\begin{gathered}
S_{Z}\left(q^{2}\right) \equiv 16 \pi \operatorname{Re}\left\{\bar{\Pi}_{T, \gamma}^{3 Q}\left(Q^{2}\right)-\bar{\Pi}_{T, Z}^{33}\left(q^{2}\right)-2 \bar{s}^{2}\left(Q^{2}\right)\left[\bar{\Pi}_{T, \gamma}^{3 Q}\left(Q^{2}\right)-\bar{\Pi}_{T, Z}^{3 Q}\left(q^{2}\right)\right]\right. \\
\left.+\bar{s}^{4}\left(Q^{2}\right)\left[\bar{\Pi}_{T, \gamma}^{Q Q}\left(Q^{2}\right)-\bar{\Pi}_{T, Z}^{Q Q}\left(q^{2}\right)\right]\right\}, \\
S_{W}\left(q^{2}\right) \equiv 16 \pi \operatorname{Re}\left[\bar{\Pi}_{T, \gamma}^{3 Q}\left(Q^{2}\right)-\bar{\Pi}_{T, W}^{11}\left(q^{2}\right)_{\hat{s}^{2}=\bar{s}^{2}\left(Q^{2}\right)}\right],
\end{gathered}
$$

remain small (free of large logarithm) at all $q^{2}, 0<\left|q^{2}\right|<\infty$.

In principle, the parametrization (B.16) can be used to account for the hadronic contributions to the $\bar{\Pi}_{T, Z}^{33}\left(q^{2}\right)$ and $\bar{\Pi}_{T, W}^{11}\left(q^{2}\right)$ terms at $\left|q^{2}\right|<m_{Z}^{2}$ with the help of the CVC and PCAC hypotheses. However, we find that the contribution of light hadrons are negligible at low momentum transfers $\left|q^{2}\right| \ll m_{Z}^{2}$, and hence the perturbative expressions (A.9) with the light quark masses as obtained by the matching conditions $(\mathbb{B . 2 0})$ and $(\mathbb{B . 2 1})$ are used when evaluating these functions.

It is important to note that the expressions $(\mathbb{B . 3 9})$ and $(\mathbb{B . 4 0})$ are valid in the sense of a perturbative expressions, and therefore the scale $Q^{2}$ has been chosen such that the $S_{Z}\left(q^{2}\right)$ and $S_{W}\left(q^{2}\right)$ terms remain small. The typical scale of the charge form factors $\bar{g}_{Z}^{2}\left(q^{2}\right)$ and $\bar{g}_{W}^{2}\left(q^{2}\right)$ are $Q^{2}=m_{Z}^{2}$ rather than $Q^{2}=q^{2}$ for $\left|q^{2}\right| \ll m_{Z}^{2}$. Our definitions of the $S$ and $U$ parameters then follow

$$
\begin{aligned}
S & =S_{Z}(0)=16 \pi \operatorname{Re}\left[\bar{\Pi}_{T, \gamma}^{3 Q}\left(m_{Z}^{2}\right)-\bar{\Pi}_{T, Z}^{33}(0)\right], \\
S+U & =S_{W}(0)=16 \pi \operatorname{Re}\left[\bar{\Pi}_{T, \gamma}^{3 Q}\left(m_{Z}^{2}\right)-\bar{\Pi}_{T, W}^{11}(0)_{\hat{s}^{2}=\bar{s}^{2}\left(m_{Z}^{2}\right)}\right] .
\end{aligned}
$$




\section{App. C SM contributions to $S, T, U$ and $\bar{\delta}_{b}\left(m_{Z}^{2}\right)$}

This Appendix deals with the SM contributions to the universal electroweak parameters $S, T, U$ and the $Z b_{L} b_{L}$ vertex form factor $\bar{\delta}_{b}\left(m_{Z}^{2}\right)$ used as free fit parameters. The complete analytic formulae are given at one-loop level and the two-loop corrections are also included as far as they are known. We adopt the perturbative order $\alpha \alpha_{s}$ 46, 52,54 56] corrections at $\alpha_{s}=\alpha_{s}\left(m_{Z}\right)_{\overline{\mathrm{MS}}}$ in evaluating the $S, T, U$ and $\bar{\delta}_{b}\left(m_{Z}^{2}\right)$ parameters, since it allows the readers to reproduce our results unambiguously and straightforwardly. The effects due to non-perturbative threshold corrections [60 62] should be evaluated carefully, and one can obtain more precise predictions of the SM from our formulae by adjusting the effective top-quark mass to produce the same $S, T, U$ and $\bar{\delta}_{b}\left(m_{Z}^{2}\right)$ values.

\section{C.1 $S_{\mathrm{SM}}$}

The $S$ parameter in the SM can be expressed as a sum of three pieces:

$$
S_{\mathrm{SM}}=S_{\ell}+S_{q}+S_{B}
$$

where the indices denote contributions from the leptonic, hadronic and the bosonic (that is, $W, Z, H)$ sectors of the $\mathrm{SM}$, respectively. Each term is separately finite. $S_{\ell}$ and $S_{B}$ are given at one-loop order, whereas the hadronic contribution $S_{q}$ with the two-loop $O\left(\alpha \alpha_{s}\right)$ correction 52, 55, 56.

The leptonic contribution is a sum of three terms

$$
S_{\ell}=\frac{1}{\pi} \sum_{i=1}^{3} G_{S}^{\ell}\left(\frac{m_{\ell_{i}}^{2}}{m_{Z}^{2}}\right),
$$

where each generation $\left(\nu_{i}, \ell_{i}\right)$ contributes

$$
G_{S}^{\ell}(x)=-\frac{1}{6}\{\ln x+(1+5 x) A(x)-10 x\} .
$$

The real function $A$ is

$$
A(x)= \begin{cases}2 \sqrt{1-4 x} \ln \frac{1+\sqrt{1-4 x}}{2 \sqrt{x}} & \text { for } 0<x<\frac{1}{4}, \\ 2 \sqrt{4 x-1} \tan ^{-1} \frac{1}{\sqrt{4 x-1}} & \text { for } x>\frac{1}{4} .\end{cases}
$$

For the case of charged lepton masses much smaller than the $Z$ mass one finds

$$
G_{S}^{\ell}(x)=\left(2+\frac{1}{2} \ln x\right) x+O\left(x^{2}\right) .
$$

The $\left(\nu_{\tau}, \tau\right)$ doublet contribution is hence $S_{\ell} \approx-0.0002$. 
The hadronic contribution calculated up to $O\left(\alpha \alpha_{s}\right)$ two-loop level is [52, 55, 56]:

$$
S_{q}=S_{q}^{(0)}+S_{q}^{(1)}
$$

The one-loop contribution is again a sum of the three terms

$$
S_{q}^{(0)}=\frac{C_{q}}{\pi} \sum_{i=1}^{3} G_{S}^{q}\left(\frac{m_{u_{i}}^{2}}{m_{Z}^{2}}, \frac{m_{d_{i}}^{2}}{m_{Z}^{2}}\right)
$$

with $C_{q}=3$, where each quark generation contributes

$$
G_{S}^{q}(x, y)=\frac{1}{18}\left\{\ln \frac{y}{x}-(1+11 x) A(x)+(1-7 y) A(y)+22 x+14 y\right\} .
$$

When both quarks are light as compared to $Z$, one finds

$$
G_{S}^{q}(x, y)=\left(\frac{4}{3}+\frac{1}{2} \ln x\right) x+\left(\frac{2}{3}+\frac{1}{2} \ln y\right) y+O\left(x^{2}, y^{2}\right) .
$$

When only the down-type quark is light $(y \ll 1)$, one finds

$$
\begin{aligned}
G_{S}^{q}(x, y) & =\frac{1}{18}\left\{22 x-\ln x-(1+11 x) A(x)+3 y(4+3 \ln y)+O\left(y^{2}\right)\right\}, \\
& =\frac{1}{18}\left\{-\ln x-\frac{1}{6}+\frac{7}{20 x}+3 y(4+3 \ln y)+O\left(\frac{1}{x^{2}}, y^{2}\right)\right\} .
\end{aligned}
$$

For large $m_{t}$, the quark contribution $S_{q}$ becomes negative and its magnitude grows logarithmically.

The two-loop $O\left(\alpha \alpha_{s}\right)$ correction [52,55, 56] can be expressed in terms of the $B_{V}^{\prime}$ and $B_{A}^{\prime}$ functions (A.12) of appendix A :

$$
\begin{aligned}
S_{q}^{(1)}=C_{q} \frac{\alpha_{s}}{\pi^{2}} \sum_{i=1}^{3}\{ & \frac{5}{36} B_{V}^{\prime}\left(m_{Z}^{2} ; u_{i}, u_{i}\right)+\frac{1}{36} B_{V}^{\prime}\left(m_{Z}^{2} ; d_{i}, d_{i}\right) \\
& \left.-\frac{1}{12} B_{A}^{\prime}\left(m_{Z}^{2} ; u_{i}, u_{i}\right)-\frac{1}{12} B_{A}^{\prime}\left(m_{Z}^{2} ; d_{i}, d_{i}\right)\right\},
\end{aligned}
$$

where the quark label stands for its mass as in appendix A. It is easily seen that the righthand side of the above equation is independent of the unit-of-mass $\mu$ for each generation, and that they are in fact a function of the ratios $m_{u_{i}}^{2} / m_{Z}^{2}$ and $m_{d_{i}}^{2} / m_{Z}^{2}$. The contributions from the first two quark generations are again negligible. The two-loop term $S_{q}^{(1)}$ is hence dominated by the $(t, b)$ doublet contribution, which can be approximated by

$$
\begin{aligned}
S_{q}^{(1)} & \approx C_{q} \frac{\alpha_{s}}{\pi^{2}}\left\{-\frac{1}{18} \ln x_{t}+\frac{5 x_{t}}{9} V_{1}\left(\frac{1}{4 x_{t}}\right)-\frac{x_{t}}{3}\left[A_{1}\left(\frac{1}{4 x_{t}}\right)-A_{1}(0)\right]\right\} \\
& =C_{q} \frac{\alpha_{s}}{\pi^{2}}\left\{-\frac{1}{18} \ln x_{t}+\frac{2}{9} \zeta_{3}+\frac{1}{9}+O\left(\frac{1}{x_{t}}\right)\right\}
\end{aligned}
$$


with $x_{t} \equiv m_{t}^{2} / m_{Z}^{2}$. The expression (C.13) agrees with ref. 52. The following table shows the full hadronic contribution $S_{q}$ for several values of $m_{t}$ in lowest order $\left(\alpha_{s}=0\right)$ and with the $O\left(\alpha \alpha_{s}\right)$ corrections for $\alpha_{s}=0.12$ :

\begin{tabular}{|c|r|r|}
\hline$m_{t}(\mathrm{GeV})$ & \multicolumn{2}{|c|}{$S_{q}$} \\
\cline { 2 - 3 } & $\alpha_{s}=0$ & $\alpha_{s}=0.12$ \\
\hline 100 & -0.008 & 0.010 \\
120 & -0.033 & -0.017 \\
140 & -0.052 & -0.038 \\
160 & -0.069 & -0.055 \\
180 & -0.083 & -0.070 \\
200 & -0.095 & -0.082 \\
\hline
\end{tabular}

using for the bottom mass $m_{b}=4.7 \mathrm{GeV}$. The two-loop correction is important for relatively small $m_{t}$ values.

The bosonic contribution is expressed as

$$
S_{B}=\frac{1}{\pi}\left\{F_{S}\left(\frac{m_{W}^{2}}{m_{Z}^{2}}\right)+H_{S}\left(\frac{m_{H}^{2}}{m_{Z}^{2}}\right)\right\},
$$

where

$$
\begin{aligned}
F_{S}\left(c^{2}\right) & =-\frac{1}{12} \ln c^{2}-\frac{7}{8}-\frac{14}{3} c^{2}+\left(\frac{7}{3} c^{2}-\frac{1}{12}\right) A\left(c^{2}\right) \\
& =-1.451 \quad \text { for } \quad c^{2}=\left(\frac{80.24}{91.187}\right)^{2}, \\
H_{S}(x) & =\frac{3}{8} x-\frac{1}{12} x^{2}+\left[\frac{3-x}{4}+\frac{x^{2}}{24}+\frac{3}{4(1-x)}\right] x \ln x+\left[1-\frac{x}{3}+\frac{x^{2}}{12}\right] B(x) .
\end{aligned}
$$

Here $A(x)$ is given by (C.4), and

$$
B(x)=\left\{\begin{array}{lll}
\sqrt{x(4-x)} \tan ^{-1} \sqrt{\frac{4}{x}-1} & \text { for } & 0<x<4, \\
\sqrt{x(x-4)} \ln \frac{2}{\sqrt{x}+\sqrt{x-4}} & \text { for } \quad x>4 .
\end{array}\right.
$$

For large $m_{H}$ one has

$$
H_{S}\left(\frac{m_{H}^{2}}{m_{Z}^{2}}\right)=\frac{1}{12} \ln \frac{m_{H}^{2}}{m_{Z}^{2}}+\frac{37}{36}-\frac{17}{48} \frac{m_{Z}^{2}}{m_{H}^{2}}+O\left(\frac{m_{Z}^{4}}{m_{H}^{4}}\right) .
$$

The total bosonic contribution $S_{B}$ is tabulated below for several $m_{H}$ values:

\begin{tabular}{|c|c|}
\hline$m_{H}(\mathrm{GeV})$ & $S_{B}$ \\
\hline 50 & -0.234 \\
100 & -0.166 \\
200 & -0.107 \\
400 & -0.061 \\
1000 & -0.008 \\
\hline
\end{tabular}




\section{C.2 $T_{\mathrm{SM}}$}

The $T$ parameter in the SM can be expressed as a sum of three individually finite pieces:

$$
T_{\mathrm{SM}}=T_{\ell}+T_{q}+T_{B}
$$

where the indices denote the leptonic, the hadronic and the bosonic (that is, $\gamma, W, Z, H$ ) contributions, respectively. $T_{\ell}$ and $T_{B}$ are evaluated at one-loop order, whereas $T_{q}$ contains irreducible two-loop contributions in $\alpha \alpha_{s}$ order [52,54, 56] and in the $m_{t}^{4}$ order [47,57, 58]. Reducible higher-oder contributions [63] are taken account of by the identity (2.36a).

The leptonic contribution is a sum of three terms

$$
T_{\ell}=\frac{G_{F} m_{Z}^{2}}{2 \sqrt{2} \pi^{2} \alpha} \sum_{i=1}^{3} G_{T}\left(0, \frac{m_{\ell_{i}}^{2}}{m_{Z}^{2}}\right)
$$

where

$$
G_{T}(x, y)=\frac{x+y}{4}+\frac{x y}{2(x-y)} \ln \frac{y}{x}
$$

The leptonic contribution of the first three generations is hence negligible; even the $\left(\nu_{\tau}, \tau\right)$ doublet contributes to $T_{\ell}$ only about 0.00005 .

The hadronic contribution is calculated including the $O\left(\alpha \alpha_{s}\right)$ [52, 54 56] and the irreducible $O\left(m_{t}^{4}\right)$ 47,57,58 two-loop corrections:

$$
T_{q}=T_{q}^{(0)}+T_{q}^{(1)}+T_{q}^{(2)}
$$

The one-loop contribution is a sum of the nine terms

$$
T_{q}^{(0)}=\frac{G_{F} m_{Z}^{2}}{2 \sqrt{2} \pi^{2} \alpha} C_{q} \sum_{i, j=1}^{3}\left|V_{u_{i}, d_{j}}\right|^{2} G_{T}\left(\frac{m_{u_{i}}^{2}}{m_{Z}^{2}}, \frac{m_{d_{j}}^{2}}{m_{Z}^{2}}\right),
$$

with $C_{q}=3$. The function $G_{T}(x, y)$ is found in (C.22). In the limit of the diagonal KM matrix elements $V_{i j}=\delta_{i j}$, the contributions from the light quarks of the first and second generations can be neglected.

The two-loop contributions are only important for the $t$ - $b$ doublet :

$$
\begin{aligned}
& T_{q}^{(1)}=-C_{q} \frac{\alpha_{s}}{\pi} \cdot \frac{3+\pi^{2}}{18}\left(\frac{m_{t}^{2}}{m_{Z}^{2}}\right)\left(\frac{G_{F} m_{Z}^{2}}{2 \sqrt{2} \pi^{2} \alpha}\right) \\
& T_{q}^{(2)}=C_{q} \alpha\left(\frac{m_{t}^{2}}{4 m_{Z}^{2}}\right)^{2}\left(\frac{G_{F} m_{Z}^{2}}{2 \sqrt{2} \pi^{2} \alpha}\right)^{2} \rho^{(2)}\left(m_{H} / m_{t}\right)
\end{aligned}
$$


where terms of order $m_{b}^{2} / m_{t}^{2}$ are neglected. The function $\rho^{(2)}\left(m_{H} / m_{t}\right)$ gives 47 : $\rho^{(2)}\left(m_{H} / m_{t}\right)=-0.74,-4.72,-6.95,-11.70,-10.74$, for $m_{H} / m_{t}=0, \frac{1}{2}, 1,5,10$. The numerical value of the 'expansion parameter' in the above expressions is $G_{F} m_{Z}^{2} / 2 \sqrt{2} \pi^{2} \alpha=$ 0.4761 .

The following table shows the contributions from each term in eq. (C.23) for several values of $m_{t}$, the lowest order contribution $T_{q}^{(0)}$ and the $O\left(\alpha \alpha_{s}\right)$ contribution $T_{q}^{(1)}$ with $\alpha_{s}=0.12$, and $O\left(m_{t}^{4}\right)$ contribution $T_{q}^{(2)}$ with $m_{H}=100,1000 \mathrm{GeV}$ :

\begin{tabular}{|c|c|c|c|c|}
\hline \multirow{2}{*}{$m_{t}(\mathrm{GeV})$} & \multirow{2}{*}{$T_{q}^{(0)}$} & $T_{q}^{(1)}$ & \multicolumn{2}{|c|}{$T_{q}^{(2)}$} \\
\cline { 3 - 5 } & & $\alpha_{s}=0.12$ & $m_{H}=100$ & $m_{H}=1000$ \\
\hline 100 & 0.419 & -0.047 & -0.003 & -0.005 \\
120 & 0.607 & -0.068 & -0.006 & -0.011 \\
140 & 0.830 & -0.092 & -0.010 & -0.020 \\
160 & 1.087 & -0.120 & -0.016 & -0.035 \\
180 & 1.379 & -0.152 & -0.024 & -0.055 \\
200 & 1.705 & -0.188 & -0.034 & -0.084 \\
\hline
\end{tabular}

using for the bottom mass $m_{b}=4.7 \mathrm{GeV}$ in $T_{q}^{(0)}$.

The bosonic contribution is

$$
T_{B}=\frac{G_{F} m_{Z}^{2}}{2 \sqrt{2} \pi^{2} \alpha}\left[F_{T}\left(\frac{m_{W}^{2}}{m_{Z}^{2}}\right)+H_{T}\left(\frac{m_{H}^{2}}{m_{Z}^{2}}\right)\right],
$$

where

$$
\begin{aligned}
F_{T}\left(c^{2}\right) & =\left(\frac{1}{4}+2 \hat{s}^{2}\right) \frac{c^{2} \ln c^{2}}{1-c^{2}}-\frac{3}{4} c^{2}+1-\hat{s}^{2} \\
& =-0.4371 \quad \text { for } \quad c^{2}=\left(\frac{80.24}{91.187}\right)^{2} \text { and } \hat{s}^{2}=0.2312, \\
H_{T}(x) & =\frac{3}{4} x\left[\frac{\ln x}{1-x}-\frac{\ln \left(x / c^{2}\right)}{1-x / c^{2}}\right] ; \quad c^{2} \equiv \frac{m_{W}^{2}}{m_{Z}^{2}} .
\end{aligned}
$$

For a heavy Higgs boson $\left(m_{H}^{2} \gg m_{Z}^{2}\right)$ one finds

$H_{T}\left(\frac{m_{H}^{2}}{m_{Z}^{2}}\right)=-\frac{3}{4}\left[\left(1-c^{2}\right) \ln \frac{m_{H}^{2}}{m_{Z}^{2}}+c^{2} \ln c^{2}+\left\{\left(1-c^{4}\right) \ln \frac{m_{H}^{2}}{m_{Z}^{2}}+c^{4} \ln c^{2}\right\} \frac{m_{Z}^{2}}{m_{H}^{2}}+O\left(\frac{m_{Z}^{4}}{m_{H}^{4}}\right)\right]$.

The total bosonic contribution $T_{B}$ is tabulated below for several $m_{H}$ values:

\begin{tabular}{|c|c|}
\hline$m_{H}(\mathrm{GeV})$ & $T_{B}$ \\
\hline 50 & -0.227 \\
100 & -0.257 \\
200 & -0.314 \\
400 & -0.396 \\
1000 & -0.529 \\
\hline
\end{tabular}




\section{C.3 $U_{\mathrm{SM}}$}

The $U$ parameter in the SM can be expressed as a sum of three pieces:

$$
U_{\mathrm{SM}}=U_{\ell}+U_{q}+U_{B}
$$

where the indices denote the leptonic, the hadronic and the bosonic (that is, $\gamma, W, Z, H$ ) contributions. Each term is separately finite. $U_{\ell}$ and $U_{B}$ are given at one-loop order, whereas the hadronic contribution $U_{q}$ is given with the two-loop $O\left(\alpha \alpha_{s}\right)$ correction [52, 54 56].

The leptonic contribution is a sum of three terms

$$
U_{\ell}=\frac{1}{\pi} \sum_{i=1}^{3} G_{U}\left(0, \frac{m_{\ell_{i}}^{2}}{m_{Z}^{2}}\right),
$$

where the contribution of each generation $\left(\nu_{i}, \ell_{i}\right)$ is

$$
G_{U}(x, y)=-\frac{x+y}{3}-\frac{1-x}{6} A(x)-\frac{1-y}{6} A(y)+f_{U}\left(x / c^{2}, y / c^{2}\right) .
$$

Here $A(x)$ is given in (C.4), and

$$
\begin{aligned}
f_{U}(x, y)= & \frac{x+y}{12}+\frac{(x-y)^{2}}{6}+\frac{(x-y)^{4}-3\left(x^{2}+y^{2}\right)}{12(x-y)} \ln \frac{y}{x} \\
& +\frac{(x-y)^{2}+x+y-2}{6} \beta(x, y) L(x, y),
\end{aligned}
$$

with

$$
\beta(x, y)=\sqrt{\left|1-2(x+y)^{2}+(x-y)^{2}\right|},
$$

and

$$
L(x, y)= \begin{cases}\frac{1}{2} \ln \frac{1-x-y+\beta(x, y)}{1-x-y-\beta(x, y)} & \text { for } \quad|\sqrt{x}-\sqrt{y}|>1 \text { or } \sqrt{x}+\sqrt{y}<1 \\ \tan ^{-1} \frac{1-x+y}{\beta(x, y)}+\tan ^{-1} \frac{1+x-y}{\beta(x, y)} & \text { for } \quad|\sqrt{x}-\sqrt{y}|<1<\sqrt{x}+\sqrt{y} .\end{cases}
$$

In the limit of vanishing lepton mass one has

$$
G_{U}(0,0)=\frac{1}{3} \ln c^{2}
$$

for $c^{2} \equiv m_{W}^{2} / m_{Z}^{2}$, and hence the contribution of the first three lepton generations is

$$
U_{\ell} \approx \frac{1}{\pi} \ln c^{2}=-0.0814 \text { for } c^{2}=\left(\frac{80.24}{91.187}\right)^{2} \text {. }
$$


The hadronic contribution is calculated up to two-loop $O\left(\alpha \alpha_{s}\right)$ level [52, 55, 56]:

$$
U_{q}=U_{q}^{(0)}+U_{q}^{(1)}
$$

The one-loop contribution is again a sum of the three terms in the limit of diagonal KM matrix elements $V_{i j}=\delta_{i j}$ :

$$
U_{q}^{(0)}=\frac{C_{q}}{\pi} \sum_{i=1}^{3} G_{U}\left(\frac{m_{u_{i}}^{2}}{m_{Z}^{2}}, \frac{m_{d_{i}}^{2}}{m_{Z}^{2}}\right)
$$

with $C_{q}=3$, where $G_{U}(x, y)$ has been given above in (C.35). For the first two quark generations the approximation (C.39) holds. For the contribution of the $(t, b)$ doublet the following approximation is useful:

$$
G_{U}(x, y)=\frac{1}{6} \ln x+\frac{1}{12}-\frac{1}{2} y \ln y+\left(\frac{1}{40}-\frac{1}{8} c^{2}+\frac{2}{3} y\right) \frac{1}{x}+O\left(\frac{1}{x^{2}}, y^{2}\right) .
$$

The two-loop $O\left(\alpha \alpha_{s}\right)$ correction [52, 55, 56] can be expressed by

$$
U_{q}^{(1)}=C_{q} \frac{\alpha_{s}}{\pi^{2}} \sum_{i=1}^{3} G_{U}^{(1)}\left(m_{u_{i}}, m_{d_{i}}\right),
$$

where the two-loop function $G_{U}^{(1)}$ is given in terms of the $B_{V}$ and $B_{A}$ functions (A.11) of appendix A:

$$
\begin{aligned}
G_{U}^{(1)}\left(m_{u}, m_{d}\right)=\operatorname{Re}\left\{\frac{1}{12}\left[B_{V}^{\prime}+B_{A}^{\prime}\right]\left(m_{Z}^{2} ; m_{u}, m_{u}\right)+\frac{1}{12}\left[B_{V}^{\prime}+B_{A}^{\prime}\right]\left(m_{Z}^{2} ; m_{d}, m_{d}\right)\right. \\
\left.-\frac{1}{6}\left[B_{V}^{\prime}+B_{A}^{\prime}\right]\left(m_{W}^{2} ; m_{u}, m_{d}\right)\right\} .
\end{aligned}
$$

It is readily seen that the function $G_{U}^{(1)}$ is independent of the unit-of-mass $\mu$. The contribution of the first two quark generations can be approximated by

$$
G_{U}^{(1)}(0,0)=\frac{1}{3} \ln c^{2}
$$

just like in eq. (C.39) for the one-loop contribution. The top-bottom contribution can be approximated by

$$
\begin{aligned}
G_{U}^{(1)}\left(m_{t}, 0\right)= & \frac{1}{6} \ln \frac{m_{t}^{2}}{m_{Z}^{2}}+\frac{m_{t}^{2}}{3 m_{Z}^{2}}\left[V_{1}\left(\frac{m_{Z}^{2}}{4 m_{t}^{2}}\right)+A_{1}\left(\frac{m_{Z}^{2}}{4 m_{t}^{2}}\right)-A_{1}(0)\right] \\
& -\frac{4 m_{t}^{2}}{3 m_{W}^{2}}\left[F_{1}\left(\frac{m_{W}^{2}}{m_{t}^{2}}\right)-F_{1}(0)\right] \\
= & \frac{1}{6} \ln \frac{m_{t}^{2}}{m_{Z}^{2}}-\frac{2}{3} \zeta_{3}+\frac{4}{27} \zeta_{2}+\frac{1}{6}+O\left(\frac{m_{Z}^{2}}{m_{t}^{2}}\right) .
\end{aligned}
$$


The expressions $(\overline{\text { C.46 }})$ and $(\overline{\text { C.47 }})$ agree with ref. [52]. The following table shows the total hadronic contribution $U_{q}$ for several values of $m_{t}$ in lowest order $\left(\alpha_{s}=0\right)$ and with $O\left(\alpha \alpha_{s}\right)$ corrections for $\alpha_{s}=0.12$ :

\begin{tabular}{|c|r|r|}
\hline \multirow{2}{*}{$m_{t}(\mathrm{GeV})$} & \multicolumn{2}{|c|}{$U_{q}$} \\
\cline { 2 - 3 } & $\alpha_{s}=0$ & $\alpha_{s}=0.12$ \\
\hline 100 & -0.118 & -0.148 \\
120 & -0.034 & -0.057 \\
140 & 0.029 & 0.009 \\
160 & 0.079 & 0.063 \\
180 & 0.122 & 0.108 \\
200 & 0.159 & 0.147 \\
\hline
\end{tabular}

using for the bottom mass $m_{b}=4.7 \mathrm{GeV}$ in evaluating the lowest order $G_{U}^{(0)}$, while the $O\left(\alpha \alpha_{s}\right)$ correction $G_{U}^{(1)}$ is calculated in the limit of vanishing bottom quark mass.

The bosonic contributions are given as

$$
U_{B}=\frac{1}{\pi}\left\{F_{U}\left(\frac{m_{W}^{2}}{m_{Z}^{2}}\right)+H_{U}\left(\frac{m_{H}^{2}}{m_{Z}^{2}}\right)\right\}
$$

where the constant term $F_{U}\left(m_{W}^{2} / m_{Z}^{2}\right)$ is found to be

$$
\begin{aligned}
F_{U}\left(c^{2}\right)= & \left\{-2\left(\frac{1}{1-c^{2}}+\frac{1}{c^{4}}-\frac{1}{6 c^{6}}\right) \hat{s}^{2}+\frac{1}{4}\left(\frac{1}{3}-\frac{1}{1-c^{2}}+\frac{9}{c^{2}}+\frac{7}{c^{4}}-\frac{3}{2 c^{6}}\right)\right\} \ln c^{2} \\
& +\left(c^{2}+\frac{29}{4}\right) A\left(c^{2}\right)+\left\{\left(6+\frac{4}{c^{2}}-\frac{1}{c^{4}}\right) \frac{2 \hat{s}^{2}}{3}-\left(7+\frac{2}{c^{2}}-\frac{3}{4 c^{4}}\right)\right\} B\left(\frac{1}{c^{2}}\right) \\
& -\left(3-\frac{2}{3 c^{2}}\right) \frac{\hat{s}^{2}}{c^{2}}-\left(2 c^{2}-\frac{1}{8}-\frac{19}{8 c^{2}}+\frac{3}{4 c^{4}}\right) \\
= & 1.043 \text { for } c^{2}=\left(\frac{80.24}{91.187}\right)^{2} \text { and } \hat{s}^{2}=0.2312,
\end{aligned}
$$

and the $m_{H}$ dependence is given by

$$
H_{U}\left(x_{H}\right)=-H_{S}\left(x_{H}\right)+H_{S}\left(\frac{x_{H}}{c^{2}}\right) ; x_{H} \equiv \frac{m_{H}^{2}}{m_{Z}^{2}} .
$$

The function $H_{S}\left(x_{H}\right)$ is defined in eq. (C.16b). In the large mass limit $\left(m_{H} \gg m_{Z}\right)$ the leading logarithm $\left(\ln m_{H}\right)$ of the function $H_{S}$ (see eq. (C.18) ) cancels in $H_{U}$ of eq. (C.51), and hence one has

$$
H_{U}\left(\frac{m_{H}^{2}}{m_{Z}^{2}}\right)=\frac{1}{12} \ln c^{2}+\frac{17}{48}\left(1-c^{2}\right) \frac{m_{Z}^{2}}{m_{H}^{2}}+O\left(\frac{m_{Z}^{4}}{m_{H}^{4}}\right) .
$$

Note, however, that the $m_{H}$ dependence is very small as seen from the table below showing 
the total bosonic contribution $U_{q}$ for several $m_{H}$ values:

\begin{tabular}{|c|c|}
\hline$m_{H}(\mathrm{GeV})$ & $U_{B}$ \\
\hline 50 & 0.345 \\
100 & 0.344 \\
200 & 0.341 \\
400 & 0.340 \\
1000 & 0.339 \\
\hline
\end{tabular}

C.4 $\bar{\delta}_{b}\left(m_{Z}^{2}\right)_{\mathrm{SM}}$

The $Z b_{L} b_{L}$ vertex form factor $\bar{\delta}_{b}\left(m_{Z}^{2}\right)_{\mathrm{SM}}$ in the SM is expressed by:

$$
\bar{\delta}_{b}\left(m_{Z}^{2}\right)=\bar{\delta}_{b}^{(0)}\left(m_{Z}^{2}\right)+\bar{\delta}_{b}^{(1)}\left(m_{Z}^{2}\right)+\bar{\delta}_{b}^{(2)}\left(m_{Z}^{2}\right) .
$$

The one-loop contribution

$$
\bar{\delta}_{b}^{(0)}\left(m_{Z}^{2}\right)=\Gamma_{1}^{b_{L}}\left(m_{Z}^{2}\right)+\hat{c}^{2} \bar{\Gamma}_{2}^{b_{L}}\left(m_{Z}^{2}\right)+\Gamma_{3}^{b_{L}}\left(m_{Z}^{2}\right)
$$

is calculated using the vertex functions of appendix A, which can be approximated by

$$
\bar{\delta}_{b}^{(0)} \approx-0.00076-0.00217\left(\frac{m_{t}+36}{100}\right)^{2},
$$

for $100 \mathrm{GeV}<m_{t}<250 \mathrm{GeV}$. The second term at the right-hand side of eq. (C.54) is the $O\left(\alpha_{s} m_{t}^{2}\right)$ two-loop contribution [46]:

$$
\bar{\delta}_{b}^{(1)}\left(m_{Z}^{2}\right)=\frac{\alpha_{s}}{\pi} \cdot 2\left(\frac{\pi^{2}}{3}-1\right) \frac{G_{F} m_{t}^{2}}{8 \sqrt{2} \pi^{2}} .
$$

The last term is the $O\left(m_{t}^{4}\right)$ two-loop contribution [47, 48]:

$$
\bar{\delta}_{b}^{(2)}\left(m_{Z}^{2}\right)=-2\left(\frac{G_{F} m_{t}^{2}}{8 \sqrt{2} \pi^{2}}\right)^{2} \tau^{(2)}\left(m_{H} / m_{t}\right)
$$

where the function $\tau^{(2)}\left(m_{H} / m_{t}\right)$ is given in ref. [47]. For $m_{H} / m_{t}=0, \frac{1}{2}, 1,5,10$, it gives $\tau^{(2)}\left(m_{H} / m_{t}\right)=5.71,2.46,1.47,3.69,7.92$.

The following table shows the contributions from each term in eq. (C.54), $\bar{\delta}_{b}^{(0)}\left(m_{Z}^{2}\right)$, $\frac{\alpha_{s}}{\pi} \bar{\delta}_{b}^{(1)}\left(m_{Z}^{2}\right)$ with $\alpha_{s}=0.12$, and $\bar{\delta}_{b}^{(2)}\left(m_{Z}^{2}\right)$ with $m_{H}=100,1000 \mathrm{GeV}$, for several $m_{t}$ values:

\begin{tabular}{|c|c|c|c|c|}
\hline \multirow{2}{*}{$m_{t}(\mathrm{GeV})$} & $\bar{\delta}_{b}^{(0)}\left(m_{Z}^{2}\right)$ & $\bar{\delta}_{b}^{(1)}\left(m_{Z}^{2}\right)$ & \multicolumn{2}{|c|}{$\bar{\delta}_{b}^{(2)}\left(m_{Z}^{2}\right)$} \\
\cline { 3 - 5 } & & $\alpha_{s}=0.12$ & $m_{H}=100$ & $m_{H}=1000$ \\
\hline 100 & -0.00481 & 0.00018 & -0.00000 & -0.00002 \\
120 & -0.00603 & 0.00026 & -0.00001 & -0.00003 \\
140 & -0.00746 & 0.00036 & -0.00002 & -0.00005 \\
160 & -0.00908 & 0.00047 & -0.00003 & -0.00007 \\
180 & -0.01089 & 0.00059 & -0.00005 & -0.00010 \\
200 & -0.01285 & 0.00073 & -0.00009 & -0.00013 \\
\hline
\end{tabular}




\section{App. D One loop scalar functions}

In this appendix explicit analytic expressions for the $B$ functions are given, as well as the reduction of higher $C$ and $D$ functions to $C_{0}$ and $D_{0}$ functions.

\section{D.1 $A, B, C$ and $D$ functions}

Following Passarino and Veltman [32] the $A, B, C$ and $D$ functions are defined by :

$$
\begin{aligned}
A\left(m_{i}\right) & =\int \frac{\overline{d^{D} k}}{i \pi^{2}} \frac{1}{N_{i}}, \\
{\left[B_{0}, B^{\mu}, B^{\mu \nu}\right](i j) } & =\int \frac{\overline{d^{D} k}}{i \pi^{2}} \frac{\left.1, k^{\mu}, k^{\mu} k^{\nu}\right]}{N_{i} N_{j}}, \\
{\left[C_{0}, C^{\mu}, C^{\mu \nu}\right](i j k) } & =\int \frac{\overline{d^{D} k}}{i \pi^{2}} \frac{\left[1, k^{\mu}, k^{\mu} k^{\nu}\right]}{N_{i} N_{j} N_{k}}, \\
{\left[D_{0}, D^{\mu}, D^{\mu \nu}\right](i j k \ell) } & =\int \frac{\overline{d^{D} k}}{i \pi^{2}} \frac{\left.1, k^{\mu}, k^{\mu} k^{\nu}\right]}{N_{i} N_{j} N_{k} N_{\ell}},
\end{aligned}
$$

where $D=4-2 \epsilon$,

$$
\overline{d^{D} k}=\Gamma(1-\epsilon)\left(\pi \mu^{2}\right)^{\epsilon} d^{D} k
$$

is the $\overline{\mathrm{MS}}$ regularization [70, [17], and the propagator factors are

$$
\begin{aligned}
& N_{1}=k^{2}-m_{1}^{2}+i \varepsilon, \\
& N_{2}=\left(k+p_{1}\right)^{2}-m_{2}^{2}+i \varepsilon, \\
& N_{3}=\left(k+p_{1}+p_{2}\right)^{2}-m_{3}^{2}+i \varepsilon, \\
& N_{4}=\left(k+p_{1}+p_{2}+p_{3}\right)^{2}-m_{4}^{2}+i \varepsilon .
\end{aligned}
$$

The vector/tensor functions are reduced to scalar functions as

$$
\begin{aligned}
B^{\mu}(12) & =p_{1}^{\mu} B_{1}(12), \\
B^{\mu \nu}(12) & =p_{1}^{\mu} p_{1}^{\nu} B_{21}(12)+g^{\mu \nu} B_{22}(12),
\end{aligned}
$$

for the two-point functions,

$$
\begin{aligned}
C^{\mu}(123) & =p_{1}^{\mu} C_{11}(123)+p_{2}^{\mu} C_{12}(123), \\
C^{\mu \nu}(123) & =p_{1}^{\mu} p_{1}^{\nu} C_{21}(123)+p_{2}^{\mu} p_{2}^{\nu} C_{22}(123)+p_{1}^{\{\mu} p_{2}^{\nu\}} C_{23}(123)+g^{\mu \nu} C_{24}(123),
\end{aligned}
$$

for the three-point functions, and

$$
\begin{aligned}
D^{\mu}(1234)= & p_{1}^{\mu} D_{11}(1234)+p_{2}^{\mu} D_{12}(1234)+p_{3}^{\mu} D_{13}(1234), \\
D^{\mu \nu}(1234)= & p_{1}^{\mu} p_{1}^{\nu} D_{21}(1234)+p_{2}^{\mu} p_{2}^{\nu} D_{22}(1234)+p_{3}^{\mu} p_{3}^{\nu} D_{23}(1234) \\
& +p_{1}^{\{\mu} p_{2}^{\nu\}} D_{24}(1234)+p_{1}^{\{\mu} p_{3}^{\nu\}} D_{25}(1234)+p_{2}^{\{\mu} p_{3}^{\nu\}} D_{26}(1234) \\
& +g^{\mu \nu} D_{27}(1234),
\end{aligned}
$$


for the four-point functions. Higher rank tensor functions do not appear in our applications in the 't Hooft-Feynman gauge.

The scalar functions $B_{i}, C_{i}, D_{i}$, are defined by

$$
B_{i}(12)=B_{i}\left(p_{1}^{2} ; m_{1}, m_{2}\right)
$$

for $i=0,1,21$ and 22 ,

$$
C_{i}(123)=C_{i}\left(p_{1}^{2}, p_{2}^{2},\left(p_{1}+p_{2}\right)^{2} ; m_{1}, m_{2}, m_{3}\right)
$$

for $i=0,11,12$ and $21-24$, and

$$
D_{i}(1234)=D_{i}\left(p_{1}^{2}, p_{2}^{2}, p_{3}^{2},\left(p_{1}+p_{2}+p_{3}\right)^{2},\left(p_{1}+p_{2}\right)^{2},\left(p_{2}+p_{3}\right)^{2} ; m_{1}, m_{2}, m_{3}, m_{4}\right)
$$

for $i=0,11-13$ and 21-27. The basic scalar functions $B_{0}, C_{0}$ and $D_{0}$ were obtained by 't Hooft and Veltman [118]. The Fortran code FF [119] is used for the general form of $C_{0}$ and $D_{0}$ functions. Reductions of higher $B, C, D$ functions are given in the following subsections.

\section{D.2 $B$ functions}

It is convenient to introduce the following four scalar $B$ functions in addition to $B_{0}$ and $B_{1}$ above :

$$
\begin{aligned}
& B_{2}\left(q^{2} ; m_{1}, m_{2}\right)=B_{21}\left(q^{2} ; m_{1}, m_{2}\right), \\
& B_{3}\left(q^{2} ; m_{1}, m_{2}\right)=-B_{1}\left(q^{2} ; m_{1}, m_{2}\right)-B_{2}\left(q^{2} ; m_{1}, m_{2}\right), \\
& B_{4}\left(q^{2} ; m_{1}, m_{2}\right)=-m_{1}^{2} B_{1}\left(q^{2} ; m_{2}, m_{1}\right)-m_{2}^{2} B_{1}\left(q^{2} ; m_{1}, m_{2}\right), \\
& B_{5}\left(q^{2} ; m_{1}, m_{2}\right)=A\left(m_{1}\right)+A\left(m_{2}\right)-4 B_{22}\left(q^{2} ; m_{1}, m_{2}\right) .
\end{aligned}
$$

All two-point functions of the standard model and its supersymmetric extension [120] are expressed compactly in terms of the above six $B_{\mathrm{n}}$ functions $(\mathrm{n}=0,1, . ., 5)$ being only logarithmically divergent. The ultra-violet singular factor is parametrized by :

$$
\Delta=\frac{1}{\epsilon}+\ln \mu^{2}
$$

In the $\overline{\mathrm{MS}}$ (modified minimal subtraction) renormalization scheme the singular piece $\Delta$ in these functions is simply replaced by a logarithm of the unit of mass $\mu$ :

$$
\Delta \stackrel{\overline{\mathrm{MS}}}{\longrightarrow} \ln \mu^{2} \text {. }
$$


The six $B_{\mathrm{n}}$ functions are then expressed by

$$
\begin{aligned}
& B_{0}\left(q^{2} ; m_{1}, m_{2}\right)=\Delta-F_{0}\left(q^{2} ; m_{1}, m_{2}\right), \\
& B_{1}\left(q^{2} ; m_{1}, m_{2}\right)=-\frac{1}{2} \Delta+F_{1}\left(q^{2} ; m_{1}, m_{2}\right), \\
& B_{2}\left(q^{2} ; m_{1}, m_{2}\right)=\frac{1}{3} \Delta-F_{2}\left(q^{2} ; m_{1}, m_{2}\right), \\
& B_{3}\left(q^{2} ; m_{1}, m_{2}\right)=\frac{1}{6} \Delta-F_{3}\left(q^{2} ; m_{1}, m_{2}\right), \\
& B_{4}\left(q^{2} ; m_{1}, m_{2}\right)=\frac{m_{1}^{2}+m_{2}^{2}}{2} \Delta-F_{4}\left(q^{2} ; m_{1}, m_{2}\right), \\
& B_{5}\left(q^{2} ; m_{1}, m_{2}\right)=\frac{q^{2}}{3} \Delta-F_{5}\left(q^{2} ; m_{1}, m_{2}\right),
\end{aligned}
$$

where the finite parts $F_{\mathrm{n}}$ have the following Feynman parametrizations :

$$
\begin{aligned}
& F_{0}\left(q^{2} ; m_{1}, m_{2}\right)=\int_{0}^{1} d x \ln H, \\
& F_{1}\left(q^{2} ; m_{1}, m_{2}\right)=\int_{0}^{1} d x x \ln H, \\
& F_{2}\left(q^{2} ; m_{1}, m_{2}\right)=\int_{0}^{1} d x x^{2} \ln H \\
& F_{3}\left(q^{2} ; m_{1}, m_{2}\right)=\int_{0}^{1} d x x(1-x) \ln H \\
& F_{4}\left(q^{2} ; m_{1}, m_{2}\right)=\int_{0}^{1} d x\left[(1-x) m_{1}^{2}+x m_{2}^{2}\right] \ln H, \\
& F_{5}\left(q^{2} ; m_{1}, m_{2}\right)=\int_{0}^{1} d x\left[(1-2 x)\left(m_{1}^{2}-m_{2}^{2}\right)+(1-2 x)^{2} q^{2}\right] \ln H,
\end{aligned}
$$

with

$$
H \equiv\left[(1-x) m_{1}^{2}+x m_{2}^{2}-x(1-x) q^{2}-i \epsilon\right] .
$$

Among the six $F_{\mathrm{n}}$ functions four $(\mathrm{n}=0,3,4,5)$ are symmetric under the exchange of the two masses. It is useful to introduce the antisymmetric $F$ function

$$
F_{A}\left(q^{2} ; m_{1}, m_{2}\right) \equiv F_{1}\left(q^{2} ; m_{2}, m_{1}\right)-F_{1}\left(q^{2} ; m_{1}, m_{2}\right)
$$

In terms of the two symmetric functions $F_{0}$ and $F_{3}$ and the antisymmetric function $F_{A}$ all the remaining $F_{\mathrm{n}}$ functions can be expressed compactly :

$$
\begin{aligned}
& F_{1}\left(q^{2} ; m_{1}, m_{2}\right)=\frac{1}{2}\left[F_{0}-F_{A}\right]\left(q^{2} ; m_{1}, m_{2}\right), \\
& F_{2}\left(q^{2} ; m_{1}, m_{2}\right)=\left[\frac{1}{2}\left(F_{0}-F_{A}\right)-F_{3}\right]\left(q^{2} ; m_{1}, m_{2}\right), \\
& F_{4}\left(q^{2} ; m_{1}, m_{2}\right)=\left[\frac{m_{1}^{2}+m_{2}^{2}}{2} F_{0}+\frac{m_{1}^{2}-m_{2}^{2}}{2} F_{A}\right]\left(q^{2} ; m_{1}, m_{2}\right), \\
& F_{5}\left(q^{2} ; m_{1}, m_{2}\right)=\left[q^{2}\left(F_{0}-4 F_{3}\right)+\left(m_{1}^{2}-m_{2}^{2}\right) F_{A}\right]\left(q^{2} ; m_{1}, m_{2}\right) .
\end{aligned}
$$


Therefore it is convenient to give closed analytic expressions for the three functions, $F_{0}, F_{3}$ and $F_{A}$ :

$$
\begin{aligned}
& F_{0}\left(q^{2} ; m_{1}, m_{2}\right)=\ln \left(m_{1} m_{2}\right)-\delta \ln \frac{m_{2}}{m_{1}}-2+\beta L, \\
& F_{3}\left(q^{2} ; m_{1}, m_{2}\right)=\frac{1}{6} \ln \left(m_{1} m_{2}\right)-\frac{3 \sigma-2 \delta^{2}}{6} \delta \ln \frac{m_{2}}{m_{1}}-\frac{5}{18}-\frac{\sigma-\delta^{2}}{3}+\frac{1+\sigma-2 \delta^{2}}{6} \beta L, \\
& F_{A}\left(q^{2} ; m_{1}, m_{2}\right)=-\left(\sigma-\delta^{2}\right) \ln \frac{m_{2}}{m_{1}}+\delta(1-\beta L),
\end{aligned}
$$

where

$$
\begin{aligned}
\sigma & =\frac{m_{1}^{2}+m_{2}^{2}}{q^{2}}, \\
\delta & =\frac{m_{1}^{2}-m_{2}^{2}}{q^{2}}, \\
\beta & = \begin{cases}\left(1-2 \sigma+\delta^{2}\right)^{\frac{1}{2}} & \text { for } q^{2}<\left(m_{1}-m_{2}\right)^{2} \text { or } q^{2}>\left(m_{1}+m_{2}\right)^{2}, \\
i\left(2 \sigma-\delta^{2}-1\right)^{\frac{1}{2}} & \text { for }\left(m_{1}-m_{2}\right)^{2}<q^{2}<\left(m_{1}+m_{2}\right)^{2},\end{cases}
\end{aligned}
$$

and the function $L$ is defined as

$$
L\left(q^{2} ; m_{1}, m_{2}\right)= \begin{cases}\frac{1}{2} \ln \frac{1+\beta-\sigma}{1-\beta-\sigma}-i \pi & \text { for } q^{2}>\left(m_{1}+m_{2}\right)^{2}, \\ \frac{1}{2} \ln \frac{1+\beta-\sigma}{1-\beta-\sigma} & \text { for } q^{2}<\left(m_{1}-m_{2}\right)^{2}, \\ \frac{1}{i}\left(\tan ^{-1} \frac{1-\delta}{|\beta|}+\tan ^{-1} \frac{1+\delta}{|\beta|}\right) & \text { for }\left(m_{1}-m_{2}\right)^{2}<q^{2}<\left(m_{1}+m_{2}\right)^{2} .\end{cases}
$$

Also the derivatives $F_{0}^{\prime}, F_{3}^{\prime}$ and $F_{A}^{\prime}$ are needed for certain applications. One finds

$$
\begin{aligned}
& F_{0}^{\prime}\left(q^{2} ; m_{1}, m_{2}\right)=\frac{1}{q^{2}}\left\{1+\delta \ln \frac{m_{2}}{m_{1}}-\left(\delta^{2}-\sigma\right) \frac{L}{\beta}\right\} \\
& F_{3}^{\prime}\left(q^{2} ; m_{1}, m_{2}\right)=\frac{1}{q^{2}}\left\{\frac{1}{6}-\delta^{2}+\frac{\sigma}{2}+\delta\left(\sigma-\delta^{2}\right) \ln \frac{m_{2}}{m_{1}}+\left(\frac{\sigma^{2}+\delta^{2}}{2}+\delta^{2}\left(\delta^{2}-2 \sigma\right)\right) \frac{L}{\beta}\right\} \\
& F_{A}^{\prime}\left(q^{2} ; m_{1}, m_{2}\right)=\frac{1}{q^{2}}\left\{\left(\sigma-2 \delta^{2}\right) \ln \frac{m_{2}}{m_{1}}-2 \delta+\delta\left(1-3 \sigma+2 \delta^{2}\right) \frac{L}{\beta}\right\} .
\end{aligned}
$$

The phase factors in $\beta$ and $L$ in eqs. (D.24,D.25) are required to obtain correctly the ratio $L / \beta$. In terms of the above three functions all the other $F_{\mathrm{n}}^{\prime}$ functions are expressed compactly:

$$
\begin{aligned}
& F_{1}^{\prime}\left(q^{2} ; m_{1}, m_{2}\right)=\frac{1}{2}\left[F_{0}^{\prime}-F_{A}^{\prime}\right]\left(q^{2} ; m_{1}, m_{2}\right), \\
& F_{2}^{\prime}\left(q^{2} ; m_{1}, m_{2}\right)=\left[\frac{F_{0}^{\prime}-F_{A}^{\prime}}{2}-F_{3}^{\prime}\right]\left(q^{2} ; m_{1}, m_{2}\right), \\
& F_{4}^{\prime}\left(q^{2} ; m_{1}, m_{2}\right)=\left[\frac{m_{1}^{2}+m_{2}^{2}}{2} F_{0}^{\prime}+\frac{m_{1}^{2}-m_{2}^{2}}{2} F_{A}^{\prime}\right]\left(q^{2} ; m_{1}, m_{2}\right), \\
& F_{5}^{\prime}\left(q^{2} ; m_{1}, m_{2}\right)=\left[F_{0}-4 F_{3}+q^{2}\left(F_{0}^{\prime}-4 F_{3}^{\prime}\right)+\left(m_{1}^{2}-m_{2}^{2}\right) F_{A}^{\prime}\right]\left(q^{2} ; m_{1}, m_{2}\right),
\end{aligned}
$$


The derivative of the $B_{\mathrm{n}}$ functions is found to be :

$$
\begin{aligned}
& B_{\mathrm{n}}^{\prime}\left(q^{2} ; m_{1}, m_{2}\right)=-F_{\mathrm{n}}^{\prime}\left(q^{2} ; m_{1}, m_{2}\right) \text { for } \mathrm{n}=0,2,3,4 \\
& B_{1}^{\prime}\left(q^{2} ; m_{1}, m_{2}\right)=F_{1}^{\prime}\left(q^{2} ; m_{1}, m_{2}\right) \\
& B_{5}^{\prime}\left(q^{2} ; m_{1}, m_{2}\right)=\frac{1}{3} \Delta-F_{5}^{\prime}\left(q^{2} ; m_{1}^{2}, m_{2}^{2}\right)
\end{aligned}
$$

\section{D.3 $C$ functions}

The higher $C$ functions

$$
C_{i} \equiv C_{i}\left(p_{1}^{2}, p_{2}^{2},\left(p_{1}+p_{2}\right)^{2} ; m_{1}, m_{2}, m_{3}\right)
$$

for $i=11,12$, and $21-24$ are given in terms of the $C_{0}, B_{0}$ and $B_{1}$ functions as

$$
\begin{aligned}
& \left(\begin{array}{l}
C_{11} \\
C_{12}
\end{array}\right)=X^{-1}\left(\begin{array}{c}
B_{0}^{(13)}-B_{0}^{(23)}+f_{1} C_{0} \\
B_{0}^{(12)}-B_{0}^{(13)}+f_{2} C_{0}
\end{array}\right) \\
& C_{24}=\frac{1}{4}+\frac{1}{4} B_{0}^{(23)}+\frac{m_{1}^{2}}{2} C_{0}-\frac{f_{1}}{4} C_{11}-\frac{f_{2}}{4} C_{12} \\
& \left(\begin{array}{l}
C_{21} \\
C_{23}
\end{array}\right)=X^{-1}\left(\begin{array}{c}
B_{1}^{(13)}+B_{0}^{(23)}+f_{1} C_{11}-2 C_{24} \\
B_{1}^{(12)}-B_{1}^{(13)}+f_{2} C_{11}
\end{array}\right) \\
& \left(\begin{array}{l}
C_{23} \\
C_{22}
\end{array}\right)=X^{-1}\left(\begin{array}{c}
B_{1}^{(13)}-B_{1}^{(23)}+f_{1} C_{12} \\
-B_{1}^{(13)}+f_{2} C_{12}-2 C_{24}
\end{array}\right)
\end{aligned}
$$

where

$$
X \equiv\left(\begin{array}{cc}
2 p_{1}^{2} & 2 p_{1} p_{2} \\
2 p_{1} p_{2} & 2 p_{2}^{2}
\end{array}\right)
$$

and

$$
\begin{aligned}
& f_{1}=m_{2}^{2}-m_{1}^{2}-p_{1}^{2} \\
& f_{2}=m_{3}^{2}-m_{2}^{2}-\left(p_{1}+p_{2}\right)^{2}+p_{1}^{2}
\end{aligned}
$$

Here the shorthand notations

$$
\begin{aligned}
B_{i}^{(12)} & \equiv B_{i}\left(p_{1}^{2} ; m_{1}, m_{2}\right) \\
B_{i}^{(13)} & \equiv B_{i}\left(\left(p_{1}+p_{2}\right)^{2} ; m_{1}, m_{3}\right) \\
B_{i}^{(23)} & \equiv B_{i}\left(p_{2}^{2} ; m_{2}, m_{3}\right)
\end{aligned}
$$

are used for $B_{0}$ and $B_{1}$ functions. 


\section{D.4 $D$ functions}

The higher $D$ functions

$$
D_{i} \equiv D_{i}\left(p_{1}^{2}, p_{2}^{2}, p_{3}^{2},\left(p_{1}+p_{2}+p_{3}\right)^{2},\left(p_{1}+p_{2}\right)^{2},\left(p_{1}+p_{3}\right)^{2} ; m_{1}, m_{2}, m_{3}, m_{4}\right)
$$

for $i=0,11-13$ and 21-27 are expressed in terms of the $D_{0}, C_{0}$ and $B_{0}$ functions as follows:

$$
\begin{aligned}
& \left(\begin{array}{l}
D_{11} \\
D_{12} \\
D_{13}
\end{array}\right)=X^{-1}\left(\begin{array}{l}
C_{0}^{(134)}-C_{0}^{(234)}+f_{1} D_{0} \\
C_{0}^{(124)}-C_{0}^{(134)}+f_{2} D_{0} \\
C_{0}^{(123)}-C_{0}^{(124)}+f_{3} D_{0}
\end{array}\right), \\
& D_{27}=m_{1}^{2} D_{0}+\frac{1}{2} C_{0}^{(234)}-\frac{1}{2}\left[f_{1} D_{11}+f_{2} D_{12}+f_{3} D_{13}\right] \\
& \left(\begin{array}{l}
D_{21} \\
D_{24} \\
D_{25}
\end{array}\right)=X^{-1}\left(\begin{array}{l}
C_{11}^{(134)}+C_{0}^{(234)}+f_{1} D_{11}-2 D_{27} \\
C_{11}^{(124)}-C_{11}^{(134)}+f_{2} D_{11} \\
C_{11}^{(123)}-C_{11}^{(124)}+f_{3} D_{11}
\end{array}\right) \\
& \left(\begin{array}{l}
D_{24} \\
D_{22} \\
D_{25}
\end{array}\right)=X^{-1}\left(\begin{array}{l}
C_{11}^{(134)}-C_{11}^{(234)}+f_{1} D_{12} \\
C_{12}^{(124)}-C_{11}^{(134)}+f_{2} D_{12}-2 D_{27} \\
C_{12}^{(123)}-C_{12}^{(124)}+f_{3} D_{12}
\end{array}\right) \\
& \left(\begin{array}{l}
D_{25} \\
D_{26} \\
D_{23}
\end{array}\right)=X^{-1}\left(\begin{array}{l}
C_{12}^{(134)}-C_{12}^{(234)}+f_{1} D_{13} \\
C_{12}^{(124)}-C_{12}^{(134)}+f_{2} D_{13} \\
-C_{12}^{(124)}+f_{3} D_{13}-2 D_{27}
\end{array}\right)
\end{aligned}
$$

where

$$
X \equiv\left(\begin{array}{ccc}
2 p_{1}^{2} & 2 p_{1} p_{2} & 2 p_{1} p_{3} \\
2 p_{1} p_{2} & 2 p_{2}^{2} & 2 p_{2} p_{3} \\
2 p_{1} p_{3} & 2 p_{2} p_{3} & 2 p_{3}^{2}
\end{array}\right)
$$

and

$$
\begin{aligned}
& f_{1}=m_{2}^{2}-m_{1}^{2}-p_{1}^{2} \\
& f_{2}=m_{3}^{2}-m_{2}^{2}-\left(p_{1}+p_{2}\right)^{2}+p_{1}^{2} \\
& f_{3}=m_{4}^{2}-m_{3}^{2}-\left(p_{1}+p_{2}+p_{3}\right)^{2}+\left(p_{1}+p_{2}\right)^{2} .
\end{aligned}
$$

The higher $C$ functions in eqs. (D.35) are written in terms of the $C_{0}$ and $B_{0}$ functions in analogy to the previous subsection :

$$
\begin{aligned}
& \left(\begin{array}{l}
C_{11}^{(123)} \\
C_{12}^{(123)}
\end{array}\right)=\left(\begin{array}{cc}
2 p_{1}^{2} & 2 p_{1} p_{2} \\
2 p_{1} p_{2} & 2 p_{2}^{2}
\end{array}\right)^{-1}\left(\begin{array}{c}
B_{0}^{(13)}-B_{0}^{(23)}+f_{1} C_{0}^{(123)} \\
B_{0}^{(12)}-B_{0}^{(13)}+f_{2} C_{0}^{(123)}
\end{array}\right) \\
& \left(\begin{array}{c}
C_{11}^{(124)} \\
C_{12}^{(124)}
\end{array}\right)=\left(\begin{array}{cc}
2 p_{1}^{2} & 2 p_{1}\left(p_{2}+p_{3}\right) \\
2 p_{1}\left(p_{2}+p_{3}\right) & 2\left(p_{2}+p_{3}\right)^{2}
\end{array}\right)^{-1}\left(\begin{array}{l}
B_{0}^{(14)}-B_{0}^{(24)}+f_{1} C_{0}^{(124)} \\
B_{0}^{(12)}-B_{0}^{(14)}+\left(f_{2}+f_{3}\right) C_{0}^{(124)}
\end{array}\right),
\end{aligned}
$$




$$
\begin{aligned}
& \left(\begin{array}{l}
C_{11}^{(134)} \\
C_{12}^{(134)}
\end{array}\right)=\left(\begin{array}{cc}
2\left(p_{1}+p_{2}\right)^{2} & 2\left(p_{1}+p_{2}\right) p_{3} \\
2\left(p_{1}+p_{2}\right) p_{3} & 2 p_{3}^{2}
\end{array}\right)^{-1}\left(\begin{array}{l}
B_{0}^{(14)}-B_{0}^{(34)}+\left(f_{1}+f_{2}\right) C_{0}^{(134)} \\
B_{0}^{(13)}-B_{0}^{(14)}+f_{3} C_{0}^{(134)}
\end{array}\right),(\mathrm{D} .38 \mathrm{c}) \\
& \left(\begin{array}{c}
C_{11}^{(234)} \\
C_{12}^{(234)}
\end{array}\right)=\left(\begin{array}{cc}
2 p_{2}^{2} & 2 p_{2} p_{3} \\
2 p_{2} p_{3} & 2 p_{3}^{2}
\end{array}\right)^{-1}\left(\begin{array}{l}
B_{0}^{(24)}-B_{0}^{(34)}+\left(f_{2}+2 p_{1} p_{2}\right) C_{0}^{(234)} \\
B_{0}^{(23)}-B_{0}^{(24)}+\left(f_{3}+2 p_{1} p_{3}\right) C_{0}^{(234)}
\end{array}\right),
\end{aligned}
$$

with

$$
\begin{aligned}
B_{0}^{(12)} & \equiv B_{0}\left(p_{1}^{2} ; m_{1}, m_{2}\right) \\
B_{0}^{(13)} & \equiv B_{0}\left(\left(p_{1}+p_{2}\right)^{2} ; m_{1}, m_{3}\right) \\
B_{0}^{(14)} & \equiv B_{0}\left(\left(p_{1}+p_{2}+p_{3}\right)^{2} ; m_{1}, m_{4}\right) \\
B_{0}^{(23)} & \equiv B_{0}\left(p_{2}^{2} ; m_{2}, m_{3}\right) \\
B_{0}^{(24)} & \equiv B_{0}\left(\left(p_{2}+p_{3}\right)^{2} ; m_{2}, m_{4}\right) \\
B_{0}^{(34)} & \equiv B_{0}\left(p_{3}^{2} ; m_{3}, m_{4}\right) .
\end{aligned}
$$




\section{References}

[1] B.W. Lynn, M.E. Peskin and R.G. Stuart, in Physics at LEP, ed. by J. Ellis and R. Peccei, CERN yellow report CERN 86-02 (1986), vol. 1, p. 90.

[2] D.C. Kennedy and B.W. Lynn, Nucl. Phys. B322, 1 (1989).

[3] M. Consoli, W. Hollik and F. Jegerlehner, in Z Physics at LEP 1, edited by G. Altarelli, R. Kleiss, and C. Verzegnassi, CERN 89-08, vol. 1, p. 7;

G. Burgers, F. Jegerlehner, B. Kniehl, and J. Kühn, ibid. p. 55.

[4] M. E. Peskin and T. Takeuchi, Phys. Rev. Lett. 65, 964 (1990); Phys. Rev. D46, 381 (1992).

[5] W.J. Marciano and J.L. Rosner, Phys. Rev. Lett. 65, 2963 (1990);

D.C. Kennedy and P. Langacker, Phys. Rev. Lett. 65, 2967 (1990); Phys. Rev. D44, 1591 (1991).

[6] M. Kuroda, G. Moultaka and D. Schildknecht, Nucl. Phys. B350, 25 (1991);

M. Bilenky, K. Kolodziej, M. Kuroda, and D. Schildknecht, Phys. Lett. B319, 319 (1993).

S. Dittmaier, D. Schildknecht, K. Kolodziej, and M. Kuroda, Preprint BI-TP 94/09 .

[7] G. Altarelli and R. Barbieri, Phys. Lett. B253, 161 (1991);

G. Altarelli, R. Barbieri, and S. Jadach, Nucl. Phys. B369, 3 (1992);

S. Jadach, and B.F.L. Ward, Phys. Rev. D49, 5705 (1994).

[8] R.D. Peccei and S. Peris, Phys. Rev. D44, 809 (1991).

[9] W.J. Marciano, Annu. Rev. Nucl. Part. Sci. 41, 469 (1991)

[10] B. Grinstein and M.B. Wise, Phys. Lett. B265, 326 (1991).

[11] K. Hagiwara, S. Ishihara, R. Szalapski and D. Zeppenfeld, Phys. Rev. D48, 2182 (1993).

[12] R. Barbieri, M. Frigeni and F. Caravaglios, Phys. Lett. B279, 169 (1992);

G. Altarelli, R. Barbieri, and F. Caravaglios, Phys. Lett. B314, 357 (1993);

F. Caravaglios, Nucl. Phys. B390, 265 (1993);

F. Caravaglios, Preprint IFUP-TH 3/94.

[13] J. Ellis, G.L. Fogli and E. Lisi, Nucl. Phys. B393, 3 (1993); Phys. Lett. B324, 173 (1994); CERN Preprint CREN-TH. 7261/94.

[14] G. Altarelli, R. Barbieri, and F. Caravaglios, Nucl. Phys. B405, 3 (1993).

[15] U. Amaldi, A. Böhm, L.S. Durkin, P. Langacker, A.K. Mann, W.J. Marciano, A. Sirlin and H.H. Williams, Phys. Rev. D36, 1385 (1987).

[16] G. Costa, J. Ellis, G.L. Fogli, D.V. Nanopoulos and F. Zwirner, Nucl. Phys. B297, 244 (1988). 
[17] P. Langacker, M. Luo, and A.K. Mann, Rev. Mod. Phys. 64, 87 (1992).

[18] I. Maksymyk, C.P. Burgess and D. London, Phys. Rev. D50, 529 (1994); C.P. Burgess, S. Godfrey, H. Konig, D. London, I. Maksymyk, Phys. Rev. D49, 6115 (1994); Phys. Lett. B326, 276 (1994);

P. Bamert, C.P. Burgess, Preprint MCGILL-94-27 (1994).

[19] J. Ellis, S. Kelley and D.V. Nanopoulos, Phys. Lett. B260, 131 (1991);

P. Langacker and M. Luo, Phys. Rev. D44, 817 (1991);

U. Amaldi, W. de Boer and H. Fürstenau, Phys. Lett. B260, 447 (1991);

G.G. Ross and R.G. Roberts, Nucl. Phys. B377, 571 (1992);

R. Barbieri and L.J. Hall, Phys. Rev. Lett. 68, 752 (1992).

[20] J. Hisano, H. Murayama and T. Yanagida, Phys. Rev. Lett. 69, 1014 (1992).

[21] K. Hagiwara and Y. Yamada, Phys. Rev. Lett. 70, 709 (1993);

Y. Yamada, Z. Phys. C60, 83 (1993).

[22] J. Ellis, S. Kelley and D.V. Nanopoulos, Nucl. Phys. B373, 55 (1992); Phys. Lett. B287, 95 (1992).

[23] P. Langacker and N. Polonsky, Phys. Rev. D47, 4028 (1993).

[24] J.E. Kim, P. Langacker, M. Levine and H.H. Williams, Rev. Mod. Phys. 53, 211 (1981).

[25] Particle Data Group, K. Hikasa et al., Phys. Rev. D45, Vol.11-II (1992).

[26] The LEP Collaborations, ALEPH, DELPHI, L3, OPAL and The LEP electroweak working group, CERN/PPE/93-157 (August, 1993).

[27] H. Burkhardt, F. Jegerlehner, G. Penso and C. Verzegnassi, Z. Phys. C43, 497 (1989);

F. Jegerlehner, in Proceedings of the 1990 Theoretical Advanced Study Institute in Elementary Particle Physics, ed. by P. Langacker and M. Cvetič.

[28] F. Jegerlehner, as quoted in B.A. Kniehl, Proceedings of the International Europhysics Conference on High Energy Physics, Marseille, France, July 22-28, 1993, ed. by J. Carr and M. Perrottet (Editions Frontières, Gif-sur-Yvette), p. 639.

[29] B.W. Lynn, G. Penso and C. Verzegnassi, Phys. Rev. D35, 42 (1987).

[30] G. Altarelli, in QCD-20 years later, Aachen, June 9-13,1992, World Scientific Vol. 1 (1993), p. 172.

[31] SLD Collaboration, K. Abe et al., Phys. Rev. Lett. 73, 25 (1994).

[32] G. Passarino and M. Veltman, Nucl. Phys. B160, 151 (1979).

[33] J.M. Cornwall and J. Papavassiliou, Phys. Rev. D40, 3474 (1989);

J. Papavassiliou, Phys. Rev. D41, 3179 (1990). 
[34] G. Degrassi and A. Sirlin, Nucl. Phys. B383, 73 (1992); Phys. Rev. D46, 3104 (1992);

G. Degrassi, B.A. Kniehl and A. Sirlin, Phys. Rev. D48, 3963 (1993).

[35] A. Sirlin, Phys. Lett. B232, 123 (1989);

S. Fanchiotti and A. Sirlin, Phys. Rev. D41, 319 (1990);

G. Degrassi, S. Fanchiotti and A. Sirlin, Nucl. Phys. B351, 49 (1991).

[36] S. Dawson, J.S. Hagelin and L. Hall, Phys. Rev. D23, 2666 (1981).

[37] S. Willenbrock and G. Valencia, Phys. Lett. B259, 373 (1991);

R.G. Stuart, Phys. Lett. B262, 113 (1991); Phys. Lett. B272, 353 (1991).

[38] A. Sirlin, Phys. Rev. Lett. 67, 2127 (1991); Phys. Lett. B267, 240 (1991);

A. Leike, T. Riemann and J. Rose, Phys. Lett. B273, 513 (1991); see also

D.Y. Bardin, A. Leike, T. Riemann and M. Sachwitz, Phys. Lett. B206, 539 (1988).

[39] A.A. Akhundov, D.Yu. Bardin and T. Riemann, Nucl. Phys. B276, 1 (1986);

W. Beenakker and W. Hollik, Z. Phys. C40, 141 (1988);

J. Bernabéu, A. Pich and A. Santamaria, Phys. Lett. B200, 569 (1988); Nucl. Phys. B363, 326 (1991).

[40] B.W. Lynn and R.G. Stuart, Phys. Lett. B252, 676 (1990).

[41] W. Hollik, Fort. der Physik, 38, 165 (1990).

[42] J. Fujimoto, M. Igarashi, N. Nakazawa, Y. Shimizu, K. Tobimatsu, Prog. Theor. Phys. Supplement 100 1, (1990).

[43] B.A. Kniehl and R.G. Stuart, Comput. Phys. Commun. 72, 175 (1992).

[44] W. Hollik, Precision Tests of the Standard Model, in Advanced Series on Directions in High Energy Physics, ed. by Paul Langacker (World Scientific, 1993)

[45] F. Boudjema, A. Djouadi and C. Verzegnassi, Phys. Lett. B238, 423 (1990);

A. Blondel, A. Djouadi and C. Verzegnassi, Phys. Lett. B293, 253 (1992).

[46] J. Fleischer, O.V. Tarasov, F. Jegerlehner and P. Rączka, Phys. Lett. B293, 437 (1992);

G. Buchalla and A.J. Buras, Nucl. Phys. B398, 285 (1993);

G. Degrassi, Nucl. Phys. B407, 271 (1993);

K.G. Chetyrkin, A. Kwiatkowski and M. Steinhauser, Mod. Phys. Lett. A8, 2785 (1993).

[47] R. Barbieri, M. Beccaria, P. Ciafaloni, G. Curci and A. Viceré, Phys. Lett. B288, 95 (1992); Erratum B312, 511 (1993); Nucl. Phys. B409, 105 (1993).

[48] A. Denner, W. Hollik and B. Lampe, Z. Phys. C60, 193 (1993).

[49] G. Degrassi, A. Sirlin and W.J. Marciano, Phys. Rev. D39, 287 (1989);

A. Góngora-T. and R.G. Stuart, Z. Phys. C55, 101 (1992).

[50] A. Sirlin, Phys. Rev. D22, 971 (1980). 
[51] M. Veltman, Nucl. Phys. B123, 89 (1977);

M.B. Einhorn, D.R.T. Jones and M. Veltman, Nucl. Phys. B191, 146 (1981).

[52] B.A. Kniehl, Phys. Rep. 240, 211 (1994).

[53] B.A. Kniehl and H-G Kohrs, Phys. Rev. D48, 225 (1993).

[54] A. Djouadi and C. Verzegnassi, Phys. Lett. B195, 265 (1987).

[55] B.A. Kniehl, J.H. Kühn and R.G. Stuart, Phys. Lett. B214, 621 (1988);

B.A. Kniehl, Nucl. Phys. B347, 86 (1990);

F. Halzen and B.A. Kniehl, Nucl. Phys. B353, 567 (1991).

[56] F. Halzen, B.A. Kniehl, and M.L. Stong, Z. Phys. C58, 119 (1993).

[57] J.J. van der Bij and F. Hoogeveen, Nucl. Phys. B283, 477 (1987).

[58] J. Fleischer, O.V. Tarasov and F. Jegerlehner, Phys. Lett. B319, 249 (1993).

[59] R. Barbieri, P. Ciafaloni and A. Strumia, Phys. Lett. B317, 381 (1993).

[60] B.A. Kniehl and A. Sirlin, Nucl. Phys. B371, 141 (1992); Phys. Rev. D47, 883 (1993);

S. Fanchiotti, B.A. Kniehl and A. Sirlin, Phys. Rev. D48, 307 (1993).

[61] F.J. Ynduráin, Phys. Lett. B321, 400 (1994);

M.C. Gonzalez-Garcia, F. Halzen and R.A. Vázqeuz, Phys. Lett. B322, 233 (1994).

[62] B.A. Kniehl and A. Sirlin, DESY report 93-194 (Dec. 1993), to be published in Phys. Rev. D.

[63] A. Cohen, H. Georgi and B. Grinstein, Nucl. Phys. B232, 61 (1984);

M. Consoli, W. Hollik and F. Jegerlehner, Phys. Lett. B227, 167 (1989);

H. Georgi, Nucl. Phys. B363, 301 (1991).

[64] V.A. Novikov, L.B. Okun and M.I. Vysotsky, Mod. Phys. Lett. A8, 2529 (1993); Erratum A8, 33011993.

[65] R.G. Stuart, Phys. Rev. Lett. 70, 3193 (1993);

T. Bhattacharya and S. Willenbrock, Phys. Rev. D47, 4022 (1993).

[66] S.G. Gorishny, A.L. Kataev and S.A. Larin, Phys. Lett. B259, 144 (1991).

[67] Y. Kizukuri, I. Ohba, K. Okano and Y. Yamanaka, Phys. Rev. D23, 2095 (1981);

Y. Kizukuri and H. Ozaki, Phys. Lett. B258, 427 (1991).

[68] B.A. Kniehl and J.H. Kühn, Phys. Lett. B224, 229 (1989); Nucl. Phys. B329, 547 (1990);

K.G. Chetyrkin and J.H. Kühn, Phys. Lett. B308, 127 (1993).

[69] K.G. Chetyrkin and J.H. Kühn; Phys. Lett. B248, 359 (1990);

K.G. Chetyrkin, J.H. Kühn and A. Kwiatkowski, Phys. Lett. B282, 221 (1992); 
K.G. Chetyrkin and A. Kwiatkowski, Phys. Lett. B305, 285 (1993);

B.A. Kniehl, KEK-TH-412, to be published in Int. J. Mod. A.

[70] O.V. Tarasov, A.A. Vladimirov and A.Yu. Zharkov, Phys. Lett. 93B, 429 (1980).

[71] D.V. Nanopoulos and D.A. Ross, Nucl. Phys. B157, 273 (1979);

R. Tarrach, ibid. B183, 384 (1981).

[72] W. Bernreuther and W. Wetzel, Nucl. Phys. B197, 228 (1982);

W. Wetzel, Nucl. Phys. B196, 259 (1982);

W. Bernreuther, Ann. Phys. 151, 127 (1983).

[73] N. Gray, D.J. Broadfurst, W. Grafe and K. Schilcher; Z. Phys. C48, 673 (1990);

E. Gross, B.A. Kniehl and G. Wolf, DESY 94-035, to be published in Z. Phys. C.

[74] J.H. Kühn and P.M. Zerwas, Phys. Rep. 167, 321 (1988);

K. Hagiwara, K. Kato, A.D. Martin and C.-K. Ng, Nucl. Phys. B344, 1 (1990);

Y. Sumino, K. Fujii, K. Hagiwara, H. Murayama and C.-K. Ng, Phys. Rev. D47, 56 (1993).

[75] P. Antonelli, M. Consoli and C. Corbo, Phys. Lett. 99B, 475 (1981);

M. Consoli, S.Lo Presti and L. Maiani, Nucl. Phys. B223, 474 (1983);

F. Jegerlehner, Z. Phys. C32, 425 (1986);

A.A. Akhundov, D.Yu Bardin and T. Riemann, Nucl. Phys. B276, 1 (1986);

W. Beenakker and W. Hollik, Z. Phys. C40, 141 (1988).

[76] J. Jersák, E. Laermann and P.M. Zerwas, Phys. Rev. D25, 1218 (1982);

A. Djouadi, J.H. Kühn and P.M. Zerwas, Z. Phys. C46, 411 (1990);

G. Altarelli and B. Lampe, Nucl. Phys. B391, 3 (1993).

[77] M. Martínez and F. Teubert, Preprint IFAE-UAB/94-01.

[78] G.L. Fogli and D. Haidt, Z. Phys. C40, 379 (1988).

[79] R. Beyer, Dissertation, Univ. of Hamburg, 1991;

G. Rädel and R. Beyer, Mod. Phys. Lett. A8, 1067 (1993).

[80] S.A. Blundell, W.R. Johnson and J. Sapirstein, Phys. Rev. Lett. 65, 1411 (1990).

[81] A. Sirlin and W.J. Marciano, Nucl. Phys. B189, 442 (1981).

[82] W.J. Marciano and A. Sirlin, Phys. Rev. D22, 2695 (1980).

[83] S. Sarantakos, A. Sirlin and W.J. Marciano, Nucl. Phys. B217, 84 (1983).

[84] CHARM Collaboration, J. Dorenbusch et al., Z. Phys. C41, 567 (1989);

L.A. Ahrens et al., Phys. Rev. D41, 3297 (1990);

CHARM-II Collaboration, P. Vilain et al., Phys. Lett. B281, 159 (1992);

[85] W.J. Marciano and A. Sirlin, Phys. Rev. D27, 552 (1983); ibid. D29, 75 (1984). 
[86] J.F. Wheater and C.H. Llewellyn Smith, Nucl. Phys. B208, 27 (1982); Erratum B226, 547 (1983).

[87] C.Y. Prescott et al., Phys. Lett. 84B, 524 (1979).

[88] CDF Collaboration, talk by K. Hara at 22'nd INS International Symposium on Physics with High Energy Colliders, Tokyo, Japan, Mar. 8-10, 1994 ;

D0 Collaboration, talk by Q. Zhu at 9'th Topical Workshop on Proton-Antiproton Collider Physics, Tsukuba, Japan, Oct. 18-22, 1993.

[89] W. Hollik, talk presented at the XVI International Symposium on Lepton-Photon Interactions, New York, Aug. 10-15,1993;

M. Swartz, ibid .

[90] SLD Collaboration, K. Abe et al., Phys. Rev. Lett. 70, 2515 (1993);

SLD Collaboration, R.D. Elia et al., Mod. Phys. Lett. A8, 2237 (1993).

[91] LEP electroweak working group, LEPEWWG/94-01 (CERN May 1994).

[92] T. Aziz, Preprint TIFR-EHEP/94-02 (1994).

[93] G.L. Fogli, private communication.

[94] R.M. Barnett, Phys. Rev. D14, 70 (1976);

H. Georgi, H.D. Politzer, Phys. Rev. D14, 1829 (1976).

[95] L. Wolfenstein, Nucl. Phys. B146, 477 (1978);

H. Fritzsch, Z. Phys. C1, 321 (1979);

T. Oka, Prog. Theor. Phys. 62, 193 (1979).

[96] J.D. Bjorken, Phys. Rev. D18, 3239 (1978);

M. Glück and E. Reya, Phys. Rev. Lett. 47, 1104 (1981);

S.M. Bilen'kii and G. Motz, Sov. J. Nucl. Phys. 34863 (1981).

[97] S. Fajfer and R.J. Oakes, Phys. Rev. D30, 1585 (1984);

P. Castorina and P.J. Mulders, Phys. Rev. D31, 2760 (1985).

[98] J.L. Miramontes, J. Sanchez Guillén and E. Zas, Nucl. Phys. B264, 77 (1986).

[99] C.H. Llewellyn Smith, Nucl. Phys. B228, 205 (1983).

[100] A. Denner and T. Sack, Z. Phys. C46, 653 (1990).

[101] G. Coignet, talk presented at the XVI International Symposium on Lepton-Photon Interactions, New York, Aug. 10-15,1993.

[102] J. Ellis, G.L. Fogli and E. Lisi, Phys. Lett. B274, 456 (1992); ibid. B285, 238 (1992); ibid. B292, 427 (1992); ibid. B318, 148 (1993).

[103] V.A. Novikov, L.B. Okun, M.I. Vysotsky and V.P. Yurov, Phys. Lett. B308, 123 (1993). 
[104] CDF Collaboration, F. Abe et al., Phys. Rev. D45, 3921 (1992).

[105] D0 Collaborations, S. Abachi et al., Phys. Rev. Lett. 72, 2138 (1994).

[106] CDF Collaboration, F. Abe et al., Phys. Rev. Lett. 73, 225 (1994); Preprint FERMILABPUB-94/097-E (April, 1994) .

[107] M. Shochet, talk presented at the DPF meeting of the American Physical Society (Albuquerque, 2-6 August 1994), Fermilab report FERMILAB-CONF-94/276-E (1994).

[108] Members of the CDF Collaboration and Others, Expression of interest in a future collider detector in B0, CDF/DOC/ADVISORY/PUBLIC/2436, Version 3.0 (1994).

[109] J.H. Kühn, in proceedings of the 2'nd International Workshop on Physics and Experiments with Linear $e^{+} e^{-}$Colliders, ed. by, F.A. Harris et al.(World Scientific, 1993), p. 72;

P. Igo-Kemenes, ibid. p. 95;

Y. Sumino, ibid. p. 439.

[110] K. Kang and S.K. Kang, Brown Univ. preprint BROWN-HET-940 (March, 1994).

[111] Z. Hioki, Tokushima Univ. preprint TOKUSHIMA-94-01 (1994);

Z. Hioki, R. Najima, Tokushima Univ. preprint TOKUSHIMA-94-02 (1994).

[112] V.A. Novikov, L.B. Okun, A.N. Rozanov and M.I. Vysotsky, CERN preprint CERN-TH7217-94 (1994).

[113] K.I. Aoki, Z. Hioki, R. Kawabe, M. Konuma, T. Muta, Prog. Theor. Phys. Suppl. 73, 1, (1982).

[114] K. Symanzik, Commun. Math. Phys. 34, 7 (1973);

T. Appelquist and J. Carazzone, Phys. Rev. D11, 2865 (1975);

Y. Kazama and Y.-P. Yao, Phys. Rev. D25, 1605 (1982).

[115] S. Weinberg, Phys. Lett. 91B, 51 (1980);

B. Ovrut and H.J. Schnitzer, Phys. Rev. D21, 3369 (1980)

[116] I. Antoniadis, C. Kounnas, and K. Tamvakis, Phys. Lett. 119B, 377 (1982);

G.A. Schuler, S. Sakakibara, and J.G. Körner, Phys. Lett. B194, 125 (1987).

[117] K.G. Chetyrkin, A.L. Kataev and F.V. Tkachov, Nucl. Phys. B174, 345 (1980).

[118] G. 't Hooft and M. Veltman, Nucl. Phys. B153, 365 (1979).

[119] G.J. van Oldenborgh and J.A.M. Vermaseren, Z. Phys. C46, 425 (1990);

G.J. van Oldenborgh, Comp. Phys. Comm. 66, 1 (1991).

[120] M. Drees, K. Hagiwara and A. Yamada, Phys. Rev. D45, 1725 (1992). 


\section{Table captions}

Table 1 Universal electroweak parameters of the spontaneously broken generic $\mathrm{SU}(2)_{\mathrm{L}} \times \mathrm{U}(1)_{\mathrm{Y}}$ theory. Column 2 list the 10 universal parameters, the masses of the weak bosons and the 4 charge form factors at two $q^{2}$ scales, 0 and $m_{Z}^{2}$. Column 3 contains three precisely measured quantities (the fine structure constant $\alpha$, the Fermi coupling constant $G_{F}$ and the $Z$ boson mass $m_{Z}$ ) together with their relation to the universal charge form factors. The factor $\bar{\delta}_{G}$ is explained in the text: see eq. (2.27). The last column lists those parameters which are used in fits. The 'star' marks parameters for which no direct experimental information is available.

Table 2 Three types of fits are considered. For each sector the free parameters are listed. External parameters in the fits in addition to the precisely known fine structure constant $\alpha$, the Fermi coupling constant $G_{F}$ and the $Z$ boson mass $m_{Z}$ are listed separately. The quantity $\delta_{\alpha}$ is defined as $\delta_{\alpha} \equiv 1 / \bar{\alpha}\left(m_{Z}^{2}\right)-128.72$ (2.31). The parameters $S, T, U$ are defined in eqs. (2.33) and $\bar{\delta}_{b}$ in eq. (2.22).

Table 3 Vertex form factors $\Gamma_{1}^{f}\left(q^{2}\right), \bar{\Gamma}_{2}^{f}\left(q^{2}\right)$ and $\Gamma_{3}^{f}\left(q^{2}\right)$ in the SM at $q^{2}=m_{Z}^{2}$. The definitions of the form factors are given in eq. (2.17) and their explicit forms in appendix A.2.

Table 4 Box form factors $B\left(e_{\alpha}, f_{\sigma}\right) \equiv B_{\alpha \sigma}^{e f}(s, t)$ for the process $e_{\alpha} \overline{e_{\alpha}} \rightarrow f_{\sigma} \overline{f_{\sigma}}$ in the SM at $s=-2 t=m_{Z}^{2}$. The definitions of the form factors are given in eq. (2.24) and their explicit forms in appendix A.3.

Table 5 Partial and total $Z$ widths in $\mathrm{MeV}$ units in the minimal SM for $m_{Z}=91.197 \mathrm{GeV}$, $\alpha_{s}\left(m_{Z}\right)=0.12, \delta_{\alpha}=0, \bar{\delta}_{G}=0.0055, m_{b}=4.7 \mathrm{GeV}$ and $m_{c}=1.4 \mathrm{GeV}$. See eqs. (3.15)-(3.18) in section 3.1 for details.

Table 6 Summary of all the electroweak data used in the fit, and the fit results. The $Z$ boson parameters are studied in section 4.1, the low energy neutral current experiments are studied in section 4.2, and the charged current experiments are studied in section 4.3. In addition, we use the fine structure constant $\alpha$ datum which fixes the charge form factor $\bar{e}^{2}(0) \cdot \chi^{2}$ has been calculated by taking the model-independent parametrizations of the original data as the inputs of our analysis: eqs. (4.1)-(4.2) for the $Z$ parameters (section 4.1), eq. (4.15) for the $\nu_{\mu}-q$ scattering experiments (section 4.2.1), eq. (4.19) for the $\nu_{\mu}-e$ scattering experiments (section 4.2.2), eq. (4.22) for the atomic parity violation experiments (section 4.2.3), eq. (4.33) for the $e-\mathrm{D}$ polarization asymmetry measurements (section 4.2.3), and eq. (4.38) for the $W$ mass measurements (section 4.3). 
Table 7 The SM predictions for the electroweak parameters. The column 'no-EW' is obtained by dropping all radiative corrections except in the running of $\bar{\alpha}\left(q^{2}\right)$ and $\bar{s}^{2}\left(q^{2}\right)$ due to light quarks and leptons. The column 'IBA' is obtained by dropping all vertex and box corrections except $\bar{\delta}_{G}$. In both 'no-EW' and 'IBA' cases, corrections due to the tree-level propagator effects and the external QED/QCD corrections are kept. When the predictions depend on $\alpha_{s}\left(m_{Z}\right)$, we show three representative cases for $\alpha_{s}\left(m_{Z}\right)=0.11,0.12$ and 0.13 from top to bottom. The $\chi^{2}$ values are obtained by taking account of the correlations among the errors that are presented in the text (see section 4 ). The total number of the data is 22 by counting also $\left(\alpha, G_{F}, m_{Z}\right)$, while the above three parameters are used as inputs of the SM analysis. The degree of freedom of the fit is hence $22-3=19$. 
Table 1

\begin{tabular}{|c|c|c|c|c|}
\hline $\begin{array}{l}\text { Electroweak } \\
\text { propagators }\end{array}$ & $\begin{array}{c}\text { Universal } \\
\text { parameters }\end{array}$ & $\begin{array}{c}\text { Precisely known } \\
\text { parameters }\end{array}$ & par & $\begin{array}{l}\text { Fit } \\
\text { meters }\end{array}$ \\
\hline$\gamma \mathrm{mc}$ & $\bar{e}^{2}(0) \quad \bar{e}^{2}\left(m_{Z}^{2}\right)$ & $\alpha=\bar{e}^{2}(0) / 4 \pi$ & & $*$ \\
\hline$\gamma m$ & $\bar{s}^{2}(0) \quad \bar{s}^{2}\left(m_{Z}^{2}\right)$ & & $\bar{s}^{2}(0)$ & $\bar{s}^{2}\left(m_{Z}^{2}\right)$ \\
\hline$Z v$ & $m_{Z} \quad \bar{g}_{Z}^{2}(0) \bar{g}_{Z}^{2}\left(m_{Z}^{2}\right)$ & $m_{Z}$ & $\bar{g}_{Z}^{2}(0)$ & $\bar{g}_{Z}^{2}\left(m_{Z}^{2}\right)$ \\
\hline W mor & $m_{W} \bar{g}_{W}^{2}(0) \bar{g}_{W}^{2}\left(m_{Z}^{2}\right)$ & $4 \sqrt{2} G_{F}=\frac{\bar{g}_{W}^{2}(0)}{m_{W}^{2}}\left(1+\bar{\delta}_{G}\right)$ & $\bar{g}_{W}^{2}(0)$ & $*$ \\
\hline
\end{tabular}

Table 2

\begin{tabular}{|c|c|c|c|}
\hline $\begin{array}{c}\text { Experimental } \\
\text { inputs }\end{array}$ & 6-parameter fit & 4-parameter fit & 2-parameter fit \\
\hline$\alpha, G_{F}, m_{Z}$ & (input) & (input) & (input) \\
\hline $\begin{array}{c}\text { low energy } \\
\text { neutral currents }\end{array}$ & $\bar{s}^{2}(0) \quad \bar{g}_{Z}^{2}(0)$ & $\delta_{\alpha}$ & $\begin{array}{ll:l}m_{t} & m_{H} & \delta_{\alpha}\end{array}$ \\
\hline$Z$ parameters & $\begin{array}{llll}\bar{s}^{2}\left(m_{Z}^{2}\right) & \bar{g}_{Z}^{2}\left(m_{Z}^{2}\right) & \bar{\delta}_{b}\left(m_{Z}^{2}\right) & \alpha_{s}\end{array}$ & $\begin{array}{lll:l}S & T & \bar{\delta}_{b}\left(m_{Z}^{2}\right) & \delta_{\alpha} \alpha_{s}\end{array}$ & $\begin{array}{ll:l}m_{t} & m_{H} & \delta_{\alpha} \alpha_{s}\end{array}$ \\
\hline$m_{W}$ & $\bar{g}_{W}^{2}(0)$ & $S T U$ & $\begin{array}{ll:l}m_{t} & m_{H} & \delta_{\alpha}\end{array}$ \\
\hline $\begin{array}{c}\text { number of } \\
\text { fit parameters }\end{array}$ & 6 & 4 & 2 \\
\hline $\begin{array}{l}\text { number of } \\
\text { external parameters }\end{array}$ & 1 & 2 & 2 \\
\hline
\end{tabular}


Table 3

\begin{tabular}{|c|c|c|c|}
\hline$f$ & $\Gamma_{1}^{f}$ & $\bar{\Gamma}_{2}^{f}$ & $\Gamma_{3}^{f}$ \\
\hline$\nu_{L}$ & $0.00252+0.00431 i$ & $-0.00680-0.00565 i$ & - \\
$\ell_{L}$ & $0.00185+0.00325 i$ & $-0.00680-0.00565 i$ & - \\
$\ell_{R}$ & $0.00020+0.00032 i$ & - & - \\
$u_{L}$ & $0.00203+0.00354 i$ & $-0.00680-0.00565 i$ & - \\
$u_{R}$ & $0.00009+0.00014 i$ & - & - \\
$d_{L}$ & $0.00225+0.00389 i$ & $-0.00680-0.00565 i$ & - \\
$d_{R}$ & $0.00002+0.00004 i$ & - & - \\
$b_{L}\left(m_{t}=100\right)$ & $0.00176+0.00107 i$ & $-0.00402+0.00000 i$ & $-0.00347+0.00000 i$ \\
$b_{L}\left(m_{t}=150\right)$ & $0.00141+0.00107 i$ & $-0.00261+0.00000 i$ & $-0.00763+0.00000 i$ \\
$b_{L}\left(m_{t}=200\right)$ & $0.00126+0.00107 i$ & $-0.00179+0.00000 i$ & $-0.01270+0.00000 i$ \\
\hline
\end{tabular}

Table 4

\begin{tabular}{|c|r|r|}
\hline$f$ & \multicolumn{1}{|c|}{$s B\left(e_{L}, f_{\sigma}\right)$} & \multicolumn{1}{|c|}{$s B\left(e_{R}, f_{\sigma}\right)$} \\
\hline$\nu_{L}$ & $0.00109+0.00000 i$ & $-0.00006+0.00000 i$ \\
$\ell_{L}$ & $-0.00005+0.00000 i$ & $-0.00002+0.00000 i$ \\
$\ell_{R}$ & $-0.00002+0.00000 i$ & $0.00001+0.00000 i$ \\
$u_{L}$ & $0.00104+0.00000 i$ & $-0.00003+0.00000 i$ \\
$u_{R}$ & $-0.00001+0.00000 i$ & $0.00001+0.00000 i$ \\
$d_{L}$ & $-0.00001+0.00000 i$ & $-0.00005+0.00000 i$ \\
$d_{R}$ & $0.00000+0.00000 i$ & $0.00000+0.00000 i$ \\
$b_{L}\left(m_{t}=100\right)$ & $-0.00002+0.00000 i$ & $-0.00005+0.00000 i$ \\
$b_{L}\left(m_{t}=150\right)$ & $-0.00001+0.00000 i$ & $-0.00005+0.00000 i$ \\
$b_{L}\left(m_{t}=200\right)$ & $0.00001+0.00000 i$ & $-0.00005+0.00000 i$ \\
\hline
\end{tabular}


Table 5

\begin{tabular}{|c|rrrrrr|}
\hline$m_{t}(\mathrm{GeV})$ & 150 & 150 & 175 & 175 & 200 & 200 \\
$m_{H}(\mathrm{GeV})$ & 100 & 1000 & \multicolumn{1}{c}{100} & \multicolumn{1}{c}{1000} & 100 & 1000 \\
\hline $\bar{g}_{Z}^{2}\left(m_{Z}^{2}\right)$ & 0.55516 & 0.55405 & 0.55641 & 0.55523 & 0.55784 & 0.55656 \\
$\bar{s}^{2}\left(m_{Z}^{2}\right)$ & 0.23119 & 0.23245 & 0.23040 & 0.23170 & 0.22952 & 0.23086 \\
$\bar{\delta}_{b}\left(m_{Z}^{2}\right)$ & -0.00789 & -0.00792 & -0.00994 & -0.00999 & -0.01226 & -0.01230 \\
\hline$\Gamma_{\nu}$ & 166.95 & 166.61 & 167.32 & 166.97 & 167.75 & 167.37 \\
$\Gamma_{e}=\Gamma_{\mu}$ & 83.81 & 83.59 & 84.04 & 83.80 & 84.30 & 84.04 \\
$\Gamma_{\tau}$ & 83.62 & 83.40 & 83.85 & 83.61 & 84.11 & 83.85 \\
$\Gamma_{u}$ & 299.20 & 297.94 & 300.41 & 299.09 & 301.76 & 300.35 \\
$\Gamma_{c}$ & 299.14 & 297.88 & 300.35 & 299.03 & 301.70 & 300.30 \\
$\Gamma_{d}=\Gamma_{s}$ & 382.65 & 381.28 & 383.77 & 382.34 & 385.09 & 383.56 \\
$\Gamma_{b}$ & 376.90 & 375.51 & 376.25 & 374.79 & 375.55 & 374.01 \\
$\Gamma_{h}$ & 1740.54 & 1733.87 & 1744.55 & 1737.59 & 1749.18 & 1741.78 \\
\hline$\Gamma_{Z}$ & 2492.63 & 2484.27 & 2498.44 & 2489.70 & 2505.15 & 2495.82 \\
\hline
\end{tabular}




\section{Table 6}

\begin{tabular}{|c|c|c|}
\hline \multicolumn{2}{|c|}{$Z$ parameters measurements } & (section 4.1) \\
\hline data & \multicolumn{2}{|c|}{$m_{Z}, \Gamma_{Z}, \sigma_{h}^{0}, R_{\ell}, A_{\mathrm{FB}}^{0, \ell}, P_{\tau}, A_{\mathrm{LR}}, A_{\mathrm{FB}}^{0, b}, A_{\mathrm{FB}}^{0, c}, R_{b}$} \\
\hline fit parameters & $m_{Z}$ (input), $\bar{s}^{2}\left(m_{Z}^{2}\right), \bar{g}_{Z}^{2}\left(m_{Z}^{2}\right), \bar{\delta}_{b}\left(m_{Z}^{2}\right)$ & d.o.f. $=10-4$ \\
\hline external parameter & \multicolumn{2}{|l|}{$\alpha_{s}$} \\
\hline \multicolumn{3}{|c|}{$\begin{array}{c}\bar{g}_{Z}^{2}\left(m_{Z}^{2}\right)=0.5542-0.00030 \frac{\alpha_{s}-0.12}{0.01} \pm 0.0017 \\
\bar{s}^{2}\left(m_{Z}^{2}\right)=0.2313+0.00008 \frac{\alpha_{s}-0.12}{0.01} \pm 0.0007 \rho_{\text {cor }} \\
\bar{\delta}_{b}\left(m_{Z}^{2}\right)=-0.0061-0.00430 \frac{\alpha_{s}-0.12}{0.01} \pm 0.0034 \\
\chi_{\min }^{2} /(\text { d.o.f. })=\left[2.48+\left(\frac{\alpha_{s}-0.1017}{0.0127}\right)^{2}\right] / 6\end{array}$} \\
\hline \multicolumn{2}{|c|}{ Low energy neutral current experiments } & (section 4.2$)$ \\
\hline data & \multicolumn{2}{|c|}{$\left(g_{L}^{2}, g_{R}^{2}, \delta_{L}^{2}, \delta_{R}^{2}\right),\left(\rho_{\nu e}, s_{\nu e}^{2}\right), Q_{W},\left(2 C_{1 u}-C_{1 d}, 2 C_{2 u}-C_{2 d}\right)$} \\
\hline fit parameters & $\bar{s}^{2}(0), \bar{g}_{Z}^{2}(0)$ & d.o.f. $=9-2$ \\
\hline \multicolumn{3}{|c|}{$\left.\begin{array}{l}\bar{g}_{Z}^{2}(0)=0.5462 \pm 0.0036 \\
\bar{s}^{2}(0)=0.2353 \pm 0.0044\end{array}\right\} \quad \rho_{\text {corr }}=0.53$} \\
\hline \multicolumn{2}{|c|}{ Charged current experiments } & (section 4.3) \\
\hline data & $G_{F}, m_{W}$ & \\
\hline fit parameters & $G_{F}$ (input), $\bar{g}_{W}^{2}(0)$ & d.o.f. $=2-2$ \\
\hline $\bar{g}_{W}^{2}(0)=0$ & $\begin{array}{l}1225-0.0031 \frac{\bar{\delta}_{G}-0.0055}{\alpha} \pm 0.0017 \\
\chi_{\min }^{2} /(\text { d.o.f. })=0 / 0\end{array}$ & \\
\hline
\end{tabular}


Table 7

\begin{tabular}{|c|c|c|c|c|c|c|c|c|}
\hline & data & no-EW & IBA & Exact & \multicolumn{4}{|c|}{ SM } \\
\hline$m_{t}(\mathrm{GeV})$ & & & 175 & 175 & 175 & 175 & 150 & 150 \\
\hline$m_{H}(\mathrm{GeV})$ & & & 100 & 100 & 60 & 1000 & 60 & 1000 \\
\hline$S$ & & & -0.2323 & -0.2323 & -0.2832 & -0.0749 & -0.2638 & -0.0555 \\
\hline$T$ & & 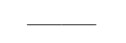 & 0.8869 & 0.8869 & 0.9174 & 0.5875 & 0.6136 & 0.3002 \\
\hline$U$ & & 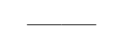 & 0.3577 & 0.3577 & 0.3591 & 0.3529 & 0.2993 & 0.2931 \\
\hline $\bar{\delta}_{G}$ & & & 0.0055 & 0.0055 & 0.0055 & 0.0055 & 0.0055 & 0.0055 \\
\hline $1 / \bar{\alpha}\left(m_{Z}^{2}\right)$ & & 128.85 & 128.71 & 128.71 & 128.71 & 128.71 & 128.72 & 128.72 \\
\hline $\bar{s}^{2}\left(m_{Z}^{2}\right)$ & & 0.2312 & 0.2304 & 0.2304 & 0.2301 & 0.2317 & 0.2309 & 0.2325 \\
\hline $\bar{g}_{Z}^{2}\left(m_{Z}^{2}\right)$ & & 0.5486 & 0.5564 & 0.5564 & 0.5564 & 0.5552 & 0.5552 & 0.5540 \\
\hline $\bar{\delta}_{b}\left(m_{Z}^{2}\right)$ & & & 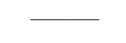 & -0.0099 & -0.0100 & -0.0100 & -0.0079 & -0.0079 \\
\hline $\bar{s}^{2}(0)$ & & 0.2388 & 0.2389 & 0.2389 & 0.2386 & 0.2401 & 0.2394 & 0.2408 \\
\hline $\bar{g}_{Z}^{2}(0)$ & & 0.5486 & 0.5492 & 0.5492 & 0.5493 & 0.5480 & 0.5481 & 0.5468 \\
\hline $\bar{g}_{W}^{2}(0)$ & & 0.4218 & 0.4242 & 0.4242 & 0.4245 & 0.4224 & 0.4229 & 0.4208 \\
\hline$\Gamma_{Z}(\mathrm{GeV})$ & $2.489 \pm 0.007$ & 2.481 & 2.519 & 2.493 & 2.494 & 2.484 & 2.488 & 2.479 \\
\hline & & 2.487 & 2.524 & 2.498 & 2.499 & 2.490 & 2.493 & 2.484 \\
\hline & & 2.493 & 2.530 & 2.504 & 2.505 & 2.495 & 2.499 & 2.490 \\
\hline$\sigma_{h}^{0}(\mathrm{nb})$ & $41.56 \pm 0.14$ & 41.53 & 41.53 & 41.52 & 41.52 & 41.52 & 41.50 & 41.51 \\
\hline & & 41.47 & 41.47 & 41.46 & 41.46 & 41.47 & 41.45 & 41.46 \\
\hline & & 41.42 & 41.42 & 41.41 & 41.41 & 41.42 & 41.39 & 41.40 \\
\hline$R_{\ell}$ & $20.763 \pm 0.049$ & 20.734 & 20.747 & 20.689 & 20.693 & 20.665 & 20.701 & 20.673 \\
\hline & & 20.801 & 20.814 & 20.756 & 20.760 & 20.732 & 20.769 & 20.741 \\
\hline & & 20.869 & 20.880 & 20.823 & 20.827 & 20.799 & 20.836 & 20.808 \\
\hline$A_{\mathrm{FP}}^{0, \ell}$ & $0.0158 \pm 0.0018$ & 0.0167 & 0.0182 & 0.0167 & 0.0171 & 0.0144 & 0.0157 & 0.0132 \\
\hline$P_{\tau}$ & $-0.139 \pm 0.014$ & -0.149 & -0.156 & -0.148 & -0.150 & -0.138 & -0.144 & -0.132 \\
\hline$A_{\mathrm{LR}}$ & $0.1637 \pm 0.0075$ & 0.1494 & 0.1557 & 0.1480 & 0.1500 & 0.1378 & 0.1438 & 0.1318 \\
\hline$R_{b}$ & $0.2203 \pm 0.0027$ & 0.2183 & 0.2182 & 0.2157 & 0.2156 & 0.2157 & 0.2165 & 0.2165 \\
\hline & & 0.2183 & 0.2182 & 0.2157 & 0.2157 & 0.2157 & 0.2165 & 0.2166 \\
\hline & & 0.2183 & 0.2182 & 0.2157 & 0.2157 & 0.2157 & 0.2165 & 0.2166 \\
\hline$A_{\mathrm{FB}}^{0, b}$ & $0.099 \pm 0.006$ & 0.105 & 0.109 & 0.104 & 0.105 & 0.096 & 0.101 & 0.092 \\
\hline & & 0.105 & 0.109 & 0.104 & 0.105 & 0.096 & 0.101 & 0.092 \\
\hline & & 0.105 & 0.109 & 0.104 & 0.105 & 0.097 & 0.101 & 0.092 \\
\hline$A_{\mathrm{FB}}^{0, c}$ & $0.075 \pm 0.015$ & 0.075 & 0.078 & 0.074 & 0.075 & 0.069 & 0.072 & 0.065 \\
\hline & & 0.075 & 0.078 & 0.074 & 0.075 & 0.069 & 0.072 & 0.065 \\
\hline & & 0.075 & 0.078 & 0.074 & 0.075 & 0.069 & 0.072 & 0.066 \\
\hline$\chi^{2}$ & $\left(\alpha_{s}=0.11\right)$ & 7.65 & 26.38 & 11.16 & 11.00 & 19.88 & 10.78 & 29.21 \\
\hline & $\left(\alpha_{s}=0.12\right)$ & 7.40 & 35.10 & 10.71 & 10.94 & 16.35 & 10.15 & 25.10 \\
\hline & $\left(\alpha_{s}=0.13\right)$ & 12.87 & 49.38 & 15.76 & 16.39 & 18.31 & 15.09 & 26.55 \\
\hline$g_{L}^{2}$ & $0.2980 \pm 0.0044$ & 0.2887 & 0.2893 & 0.2995 & 0.2998 & 0.2973 & 0.2979 & 0.2955 \\
\hline$g_{R}^{2}$ & $0.0307 \pm 0.0047$ & 0.0302 & 0.0303 & 0.0295 & 0.0295 & 0.0297 & 0.0295 & 0.0298 \\
\hline$\delta_{L}^{2}$ & $-0.0589 \pm 0.0237$ & -0.0588 & -0.0589 & -0.0634 & -0.0634 & -0.0634 & -0.0633 & -0.0632 \\
\hline$\delta_{R}^{2}$ & $0.0206 \pm 0.0160$ & 0.0181 & 0.0182 & 0.0177 & 0.0177 & 0.0178 & 0.0177 & 0.0178 \\
\hline$\chi^{2}$ & & 6.91 & 6.09 & 0.24 & 0.29 & 0.25 & 0.19 & 0.78 \\
\hline$s_{e f f}^{2}$ & $0.233 \pm 0.008$ & 0.239 & 0.239 & 0.230 & 0.230 & 0.231 & 0.231 & 0.232 \\
\hline$\rho_{\text {eff }}$ & $1.007 \pm 0.028$ & 1.000 & 1.001 & 1.013 & 1.013 & 1.011 & 1.011 & 1.009 \\
\hline$\chi^{2}$ & & 0.61 & 0.60 & 0.18 & 0.21 & 0.06 & 0.11 & 0.02 \\
\hline$Q_{W}$ & $-71.04 \pm 1.81$ & -74.89 & -74.98 & -73.21 & $\begin{array}{l}-73.17 \\
\end{array}$ & -73.31 & -73.17 & $\begin{array}{l}-73.30 \\
\end{array}$ \\
\hline$\chi^{2}$ & & 4.52 & 4.74 & 1.43 & 1.39 & 1.57 & 1.38 & 1.57 \\
\hline $2 C_{1 u}-C_{1 d}$ & $0.938 \pm 0.264$ & 0.709 & 0.709 & 0.723 & 0.724 & 0.717 & 0.720 & 0.713 \\
\hline $2 C_{2 u}-C_{2 d}$ & $-0.659 \pm 1.228$ & 0.081 & 0.080 & 0.104 & 0.105 & 0.096 & 0.101 & 0.092 \\
\hline$\chi^{2}$ & & 1.96 & 1.94 & 1.27 & 1.23 & 1.51 & 1.40 & 1.69 \\
\hline$m_{W}$ & $80.24 \pm 0.16$ & 79.95 & 80.39 & 80.39 & 80.42 & 80.22 & 80.27 & 80.08 \\
\hline$\chi^{2}$ & & 3.23 & 0.91 & 0.91 & 1.28 & 0.02 & 0.03 & 1.06 \\
\hline$\chi_{\text {tot }}^{2}$ & $\left(\alpha_{s}=0.11\right)$ & 24.87 & 40.66 & 15.20 & 15.40 & 23.29 & 13.88 & 34.33 \\
\hline & $\left(\alpha_{s}=0.12\right)$ & 24.62 & 49.38 & 14.74 & 15.34 & 19.76 & 13.26 & 30.22 \\
\hline & $(0-013)$ & 30.10 & 63.65 & 19.79 & 20.78 & 21.72 & 18.20 & 31.66 \\
\hline
\end{tabular}




\section{Figure captions}

Fig.1 The SM predictions for the $\left(S, T, U, \bar{\delta}_{b}\right)$ parameters defined in eqs. (2.34) and (2.22) are shown as functions of $m_{t}$ for selected $m_{H}$ values. Their closed analytic expressions are given in appendix C. $\alpha_{s}\left(m_{Z}\right)$ is set to 0.12 in the two-loop $O\left(\alpha \alpha_{s}\right)$ corrections for $S, T, U$ [54] and $\bar{\delta}_{b}\left(m_{Z}^{2}\right)$ [46].

Fig.2 The four charge form factors in the minimal SM as functions of the momentum transfer scale. The SM predictions are given for $m_{t}=100,150,200 \mathrm{GeV}$ and $m_{H}=100,1000 \mathrm{GeV}$. The parametrization [27] of the hadronic vacuum polarization contribution is used in the space-like region $\left(-m_{Z}^{2}<q^{2}<0\right)$. In the time-like region $\left(0<q^{2}<m_{Z}^{2}\right)$ only the heavy quark $(c, b)$ threshold corrections are taken into account. The light quark contributions at $\left|q^{2}\right|>m_{Z}^{2}$ are calculated in perturbative QCD by requiring continuity at $q^{2}=m_{Z}^{2}$. See appendix B for details.

Fig.3 The $Z$ total width $\Gamma_{Z}$ as function of the universal charge form factors $\bar{g}_{Z}^{2}\left(m_{Z}^{2}\right)$ and $\bar{s}^{2}\left(m_{Z}^{2}\right)$ for $\bar{\delta}_{b}\left(m_{Z}^{2}\right)=0(\mathrm{a}), \bar{\delta}_{b}\left(m_{Z}^{2}\right)=-0.01(\mathrm{~b})$ and $\bar{\delta}_{b}\left(m_{Z}^{2}\right)=-0.02(\mathrm{c})$. Three cases of $\alpha_{s}\left(m_{Z}\right)(0.11,0.12,0.13)$ are shown for each $\bar{\delta}_{b}\left(m_{Z}^{2}\right)$.

Fig.4 The hadronic cross section on the $Z$-pole $\sigma_{h}^{0}(\mathrm{LEP})$ as function of $\bar{s}^{2}\left(m_{Z}^{2}\right)$ and $\bar{\delta}_{b}\left(m_{Z}^{2}\right)$ for $\alpha_{s}\left(m_{Z}\right)=0.11,0.12,0.13$. The solid (dashed) lines are obtained for $\bar{g}_{Z}^{2}\left(m_{Z}^{2}\right)=$ $0.55(0.57)$. Here $\sigma_{h}^{0}(\mathrm{LEP})$ is defined by $12 \pi \Gamma_{e} \Gamma_{h} / m_{Z}^{2} \Gamma_{Z}^{2}$ in ref. [26]; see discussions in the text.

Fig.5 The ratio $R_{\ell} \equiv \sigma_{h}^{0}(\mathrm{LEP}) / \sigma_{\ell}^{0}(\mathrm{LEP})$ of the on- $Z$-pole cross sections as function of $\bar{s}^{2}\left(m_{Z}^{2}\right)$ and $\bar{\delta}_{b}\left(m_{Z}^{2}\right)$ for $\alpha_{s}\left(m_{Z}\right)=0.11,0.12,0.13$. The solid (dashed) lines are obtained for $\bar{g}_{Z}^{2}\left(m_{Z}^{2}\right)=0.55(0.57)$.

Fig.6 The ratio $R_{b} \equiv \sigma_{b}^{0} / \sigma_{h}^{0}$ of the $b$-quark production cross section to the hadronic cross section on the $Z$-pole as function of $\bar{s}^{2}\left(m_{Z}^{2}\right)$ and $\bar{\delta}_{b}\left(m_{Z}^{2}\right)$ for $\alpha_{s}\left(m_{Z}\right)=0.11,0.12,0.13$. The solid (dashed) lines are obtained for $\bar{g}_{Z}^{2}\left(m_{Z}^{2}\right)=0.55(0.57)$.

Fig.7 The asymmetries on the $Z$-pole as functions of the effective charge $\bar{s}^{2}\left(m_{Z}^{2}\right)$ : the leptonic forward-backward asymmetry $A_{\mathrm{FB}}^{0, \ell}(\mathrm{a})$, the left-right beam polarization asymmetry $A_{\mathrm{LR}}^{0}(\mathrm{~b})$, the $b$-quark forward-backward asymmetry $A_{\mathrm{FB}}^{0, b}(\mathrm{c})$, the forwardbackward asymmetry of the $c$-quark $A_{\mathrm{FB}}^{0, c}(\mathrm{~d})$. The solid lines ('Full') are obtained from the full helicity amplitudes (2.2) including the $\gamma$ and $Z$ exchange as well as the box contributions (which are negligibly small). The long dashed lines ('Full $-\gamma$ ') are obtained by subtracting from the full amplitudes (2.2) the real and imaginary 
parts of the $\gamma$-exchange contribution $Q_{i} Q_{j}\left[\bar{e}^{2}\left(m_{Z}^{2}\right)-i \hat{e}^{2} \Delta_{\gamma \gamma}\left(m_{Z}^{2}\right)\right] / s$. The thick dashed lines (' $Z$-only') are obtained by retaining only the $Z$-pole term, the term multiplying the $Z$-propagator factor in eq. (2.2). The dotted lines (' $Z$-only(IBA)') are obtained by using the improved Born approximation to the $Z$-exchange amplitudes. The thick dashed lines (' $Z$-only') are used in the present analysis. QCD corrections to $A_{\mathrm{FB}}^{0, b}$ and $A_{\mathrm{FB}}^{0, c}$ are calculated for $\alpha_{s}=0.12$.

Fig.8 $m_{t}$ dependence of the SM predictions for the electroweak $Z$ boson parameters. Predictions for three values of $m_{H}$ are shown by dashed lines $(60 \mathrm{GeV})$, solid lines $(300 \mathrm{GeV})$ and by dash-dotted lines $(1000 \mathrm{GeV})$, all calculated for $\alpha_{s}\left(m_{Z}\right)=0.12$. Also shown by straight lines are the mean (dotted lines) and the 1- $\sigma$ allowed ranges of the experimental data [26, 31] (see sections 4.1 and 6.2).

Fig.9 $\alpha_{s}\left(m_{Z}\right)$ dependence of the SM predictions for the electroweak $Z$ boson parameters. Predictions for three values of $m_{t}$ are shown by dashed lines $(100 \mathrm{GeV})$, solid lines $(150 \mathrm{GeV})$ and by dash-dotted lines $(200 \mathrm{GeV})$, all for $m_{H}=100 \mathrm{GeV}$. Also shown by straight lines are the mean (dotted lines) and the 1- $\sigma$ allowed ranges of the experimental data [26] (see section 4.1).

Fig.10 Relation between the model-independent parameters of the $\nu_{\mu}-q$ scattering experiments $\left(g_{L}^{2}, g_{R}^{2}\right)$ and the two universal form factors $\left(\bar{s}^{2}(0), \bar{g}_{Z}^{2}(0)\right)$. The 1- $\sigma$ contour of the present data [78 is also shown: see eq. (4.15) in section 4.2.1. The $\bar{\rho}=1$ line corresponds to $\bar{g}_{Z}^{2}(0)=4 \sqrt{2} G_{F} m_{Z}^{2}=0.5486$.

Fig.11 Relation between the $\nu(\bar{\nu})-e$ scattering cross sections per neutrino energy $\left(\sigma(\nu e) / E_{\nu}\right.$, $\left.\sigma(\bar{\nu} e) / E_{\nu}\right)$ and the two universal parameters $\left(\bar{s}^{2}(0), \bar{g}_{Z}^{2}(0)\right)$. The 1- $\sigma$ contour shows the experimental constraint: see eq. (4.19) [84] and eq. (4.20) in section 4.2.2. The $\bar{\rho}=1$ line corresponds to $\bar{g}_{Z}^{2}(0)=4 \sqrt{2} G_{F} m_{Z}^{2}=0.5486$.

Fig.12 Relation between the model-independent parameters $\left(2 C_{1 u}-C_{1 d}, 2 C_{2 u}-C_{2 d}\right)$ [24] of the $e-\mathrm{D}$ polarization asymmetry experiments and the two universal form factors $\left(\bar{s}^{2}(0), \bar{g}_{Z}^{2}(0)\right)$. The $1-\sigma$ contour of the present data [87] is also shown: see eq. (4.33) in section 4.2.4. The $\bar{\rho}=1$ line corresponds to $\bar{g}_{Z}^{2}(0)=4 \sqrt{2} G_{F} m_{Z}^{2}=0.5486$.

Fig.13 The weak charge $\left(Q_{W}\right)$ of the cesium atom ${ }_{55}^{133} \mathrm{C}_{\mathrm{s}}$ in the atomic parity violation experiments as function of the two universal parameters $\left(\bar{s}^{2}(0), \bar{g}_{Z}^{2}(0)\right)$. The $1-\sigma$ contour of the present data 80 is shown by dashed lines: see eq. (4.22) in section 4.2.3. The $\bar{\rho}=1$ line corresponds to $\bar{g}_{Z}^{2}(0)=4 \sqrt{2} G_{F} m_{Z}^{2}=0.5486$. 
Fig.14 The SM predictions for $m_{W}$ as functions of $m_{t}$ and $m_{H}$ for $\delta_{\alpha}=0, \bar{\delta}_{G}=0.0055$ and $\alpha_{s}=0.12$. The $1-\sigma$ allowed range of the present data [88] is shown by thick dashed lines: see eq. (4.38) in section 4.3 .

Fig.15 3-parameter fit to the $Z$ boson parameters: the $Z b_{L} b_{L}$ vertex form factor $\bar{\delta}_{b}\left(m_{Z}^{2}\right)$ is introduced as the third parameter of the fit in addition to the two universal charge form factors $\bar{s}^{2}\left(m_{Z}^{2}\right)$ and $\bar{g}_{Z}^{2}\left(m_{Z}^{2}\right)$ : see eq. (4.3). The 1- $\sigma$ contours are shown for three representative $\alpha_{s}\left(m_{Z}\right)$ values, 0.11 (dashed lines), 0.12 (solid lines), 0.13 (dot-dashed lines). Also shown are the $\mathrm{SM}$ predictions in the range $100 \mathrm{GeV}<m_{t}<200 \mathrm{GeV}$ and $50 \mathrm{GeV}<m_{H}<1000 \mathrm{GeV}$, which are calculated assuming $\left(\Delta \frac{1}{\alpha}\right)_{\text {hadrons }}=-3.88$ $\left(\delta_{\alpha}=0\right)$ [27] for the hadronic vacuum polarization contribution to $1 / \bar{\alpha}\left(m_{Z}^{2}\right)$.

Fig.16 Fit to the low energy neutral current data in terms of the two universal charge form factors $\bar{s}^{2}(0)$ and $\bar{g}_{Z}^{2}(0)$. 1- $\sigma$ contours are shown separately for the $\nu_{\mu}-q$ data 78 , the $\nu_{\mu}-e$ data [84], the atomic parity violation (APV) data [80], and the SLAC

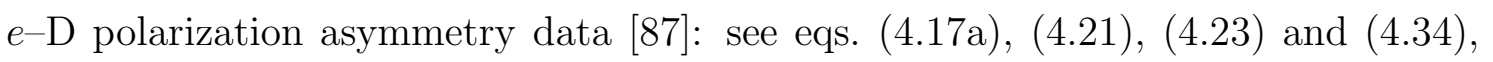
respectively. The $1-\sigma$ contour of the combined fit, eq. (4.35), is shown by the thick contour. The straight dashed line shows the 'tree' level prediction of the minimal $\mathrm{SM}: \bar{\rho} \equiv \bar{g}_{Z}^{2}(0) /\left(4 \sqrt{2} G_{F} m_{Z}^{2}\right)=1$, or $\bar{g}_{Z}^{2}(0)=4 \sqrt{2} G_{F} m_{Z}^{2}=0.5486$.

Fig.17 Fit to the SLAC $e-\mathrm{D}$ polarization asymmetry data 87 in terms of the modelindependent parameters $2 C_{1 u}-C_{1 d}$ and $2 C_{2 u}-C_{2 d}$ [24] of the effective weak Hamiltonian (3.76). Uncertainties due to the sea-quark contributions (a), the longitudinal to transverse virtual photon cross section ratio $R=\sigma_{L} / \sigma_{T}(\mathrm{~b})$, and the higher twist effects (c) have been examined, and the fit (d) is obtained after taking account of all the uncertainties. Shown by the solid lines are the tree-level predictions of the $\mathrm{SM}$, and the blobs show the predictions at selected $\sin ^{2} \theta_{W}$ values.

Fig.18 The running of the charge form factor $\bar{g}_{Z}^{2}\left(q^{2}\right), 4 \pi / \bar{g}_{Z}^{2}\left(m_{Z}^{2}\right)-4 \pi / \bar{g}_{Z}^{2}(0)$, as functions of $m_{H}$ calculated in the SM for $100 \mathrm{GeV}<m_{t}<200 \mathrm{GeV}$. The $1-\sigma$ allowed range from the neutral current experiments on the $Z$-pole and at low energies, eq. (5.1), is also shown for comparison.

Fig.19 The running of the charge form factor $\bar{g}_{Z}^{2}\left(q^{2}\right), \bar{s}^{2}\left(q^{2}\right)$ and $\bar{e}^{2}\left(q^{2}\right)$ as expected from the one-loop contribution of the wino (fermionic partner of the $W$ in the supersymmetric SM) to the three neutral current propagators. (a) $4 \pi / \bar{g}_{Z}^{2}\left(m_{Z}^{2}\right)-4 \pi / \bar{g}_{Z}^{2}(0)$; (b) $\bar{s}^{2}\left(m_{Z}^{2}\right) / \bar{\alpha}\left(m_{Z}^{2}\right)-\bar{s}^{2}(0) / \alpha$; (c) $\delta_{\alpha} \equiv 1 / \bar{\alpha}\left(m_{Z}^{2}\right)-128.72$. The SM contributions are shown for $m_{t}=100,200 \mathrm{GeV}$ and $m_{H}=60,1000 \mathrm{GeV}$. The singularity at 
$m_{\text {wino }}=m_{Z} / 2$ in (a) reflects 65 the deviation of the $Z$ line-shape from the standard Breit-Wigner form that has been assumed both in the experimental fit and in our theoretical formula. The 1- $\sigma$ allowed ranges from the neutral current experiments on the $Z$-pole and at low energies, eq. (5.1), are also shown for comparison. There is no direct measurement of $\delta_{\alpha}$.

Fig.20 2-parameter fit to the combined low energy neutral current data and the $Z$ parameters. The latter fit ('LEP+SLC') is copied from Fig. 15 for $\alpha_{s}\left(m_{Z}\right)=0.12$. The low energy combined fit of Fig. 16 has been rescaled to the $m_{Z}$ scale by assuming the SM running of the two charge form factors, $\bar{s}^{2}\left(q^{2}\right)$ and $\bar{g}_{Z}^{2}\left(q^{2}\right)$, which depend on $m_{t}$ and $m_{H}$. Uncertainties due to $m_{t}$ and $m_{H}$ in the SM predictions for the running of the form factors are illustrated by drawing the results for $m_{t}=100,200 \mathrm{GeV}$ and $m_{H}=60,1000 \mathrm{GeV}$ in the same figure. The $1-\sigma$ contour of the combined fit, eq. (5.5), is given by the thick contour, for which the above uncertainties give negligible effects.

Fig.21 Global fit to the $(S, T, U)$ parameters for three $\alpha_{s}$ values and $\delta_{\alpha} \equiv 1 / \bar{\alpha}\left(m_{Z}^{2}\right)-$ $128.72=0$ and $\bar{\delta}_{G}=0.0055$. Four $1-\sigma$ contours are obtained for each $\alpha_{s}$, by using $m_{t}=150,200 \mathrm{GeV}$ and $m_{H}=100,1000 \mathrm{GeV}$ in evaluating the running of the charge form factors: see eq. (5.8) for a parametrization of the fit for $m_{t}=175 \mathrm{GeV}$ and $m_{H}=100 \mathrm{GeV}$. The fourth parameter of the fit, the $Z b_{L} b_{L}$ vertex form factor $\bar{\delta}_{b}\left(m_{Z}^{2}\right)$, is allowed to take an arbitrary value, free from SM constraints. The SM predictions with $\delta_{\alpha}=0$ and $\bar{\delta}_{G}=0.0055$ are also given for $100 \mathrm{GeV}<m_{t}<200 \mathrm{GeV}$ and $50 \mathrm{GeV}<m_{H}<1000 \mathrm{GeV}$.

Fig.22 Electroweak constraints on $\left(m_{t}, m_{H}\right)$ in the minimal SM, for three selected $\alpha_{s}$ values at $\delta_{\alpha}=0$. Dashed lines show the best $m_{t}$ values for a given $m_{H}$, and the solid contours are for $\chi^{2}=\chi_{\min }^{2}+1$ and $\chi^{2}=\chi_{\min }^{2}+4.61$. The minimum point of $\chi^{2}$ is marked by " $\times$ ". The region $m_{H}<63 \mathrm{GeV}$ is excluded by LEP experiments [101].

Fig.23 Total $\chi^{2}$ of the SM fit to all the electroweak data as functions of $m_{t}$ for $m_{H}=60$, $300,1000 \mathrm{GeV}$ and $\alpha_{s}\left(m_{Z}\right)=0.11,0.12,0.13$. The uncertainty $\delta_{\alpha}$ in the hadronic vacuum polarization contribution to the effective charge $1 / \bar{\alpha}\left(m_{Z}^{2}\right)$ is shown for three cases, $\delta_{\alpha}=-0.1(\mathrm{a}), 0$ (b), +0.1 (c). The dotted lines are obtained by using the approximate formula (5.9). The degree of freedom is 19.

Fig.24 Total $\chi^{2}$ of the SM fit to all the electroweak data as functions of $m_{H}$ for $m_{t}=$ $120,140,160,180,200 \mathrm{GeV}$ and $\alpha_{s}\left(m_{Z}\right)=0.11,0.12,0.13$. The hadronic vacuum 
polarization contribution to the effective charge $1 / \bar{\alpha}\left(m_{Z}^{2}\right)$ is fixed by setting $\delta_{\alpha}=0$. The dotted lines show our approximation (5.9). The degree of freedom is 19.

Fig.25 The universal weak mixing form factor $\bar{s}^{2}\left(m_{Z}^{2}\right)$ as determined from various asymmetry measurements on the $Z$-pole: the lepton $(e, \mu, \tau)$ forward-backward asymmetries [26], the $\tau$ polarization asymmetry [26], the left-right asymmetry [31] and the quark $(b, c)$ forward-backward asymmetries [26]: see eqs. (4.4), (6.2) and the footnote 6. Also shown is the deviation ' $\chi$ ' (that is, $\left.\chi \equiv\left(\left\langle\bar{s}^{2}\left(m_{Z}^{2}\right)\right\rangle-0.2302\right) / \sigma\left(\bar{s}^{2}\left(m_{Z}^{2}\right)\right)\right)$ for each fit individually, where $\left\langle\bar{s}^{2}\left(m_{Z}^{2}\right)\right\rangle$ and $\sigma\left(\bar{s}^{2}\left(m_{Z}^{2}\right)\right)$ denote mean and standard deviation of each fit, respectively. At the bottom the above $\chi$-values are histogrammed.

Fig.26 Impact of the left-right asymmetry data [31] by the SLD collaboration. The band (mean (dashed line) and the 1- $\sigma$ (solid lines)) represents the constraint from the new left-right asymmetry data alone. The constraints from the fits with and without (see Figs. 15 and 21) the new asymmetry data are shown by thick and thin lines, respectively, for $\alpha_{s}\left(m_{Z}\right)=0.11,0.12,0.13$. The $Z b_{L} b_{L}$ vertex form factor $\bar{\delta}_{b}\left(m_{Z}^{2}\right)$ is allowed to take an arbitrary value, free from SM constraints.

(a): The fits are shown in the $\bar{s}^{2}\left(m_{Z}^{2}\right)-\bar{g}_{Z}^{2}\left(m_{Z}^{2}\right)$ plane: see eq. (6.5). The SM predictions are obtained by assuming $\delta_{\alpha}=0$ in the range $100 \mathrm{GeV}<m_{t}<260 \mathrm{GeV}$ and $1 \mathrm{GeV}<m_{H}<1000 \mathrm{GeV}$.

(b): The fits are shown in the $S-T$ plane, where $\delta_{\alpha}=0$ and $\bar{\delta}_{G}=0.0055$ are assumed, and $m_{t}=174 \mathrm{GeV}$ and $m_{H}=100 \mathrm{GeV}$ are used to calculate the SM running of the charge form factors between $q^{2}=0$ and $q^{2}=m_{Z}^{2}$ : see eq. (6.5) for parametrization of the result. The results are insensitive to the actual $\left(m_{t}, m_{H}\right)$ values in the region $m_{t}>100 \mathrm{GeV}$ and $m_{H}>50 \mathrm{GeV}$ (see Figs. 18 and 19). The SM predictions are given in the range $100 \mathrm{GeV}<m_{t}<260 \mathrm{GeV}$ and $50 \mathrm{GeV}<m_{H}<1000 \mathrm{GeV}$.

Fig.27 Electroweak constraints on $\left(m_{t}, m_{H}\right)$ in the minimal SM, including the new left-right asymmetry data [31, for three selected $\alpha_{s}$ values $\alpha_{s}\left(m_{Z}\right)=0.11,0.12,0.13$, and for (a) $\delta_{\alpha}=-0.1$, (b) $\delta_{\alpha}=0$, and (c) $\delta_{\alpha}=0.1$. Dashed lines show the best $m_{t}$ values for a given $m_{H}$, and the solid contours are for $\chi^{2}=\chi_{\min }^{2}+1$ and $\chi^{2}=\chi_{\min }^{2}+4.61$. The minimum point of $\chi^{2}$ is marked by " $\times$ ". The region $m_{H}<63 \mathrm{GeV}$ is excluded by LEP experiments 101.

Fig.28 Total $\chi^{2}$ of the SM fit to all the electroweak data including the new left-right asymmetry data [31] as functions of $m_{t}$ for $m_{H}=60,300,1000 \mathrm{GeV}$ and $\alpha_{s}\left(m_{Z}\right)=0.11$, $0.12,0.13$. The uncertainty $\delta_{\alpha}$ in the hadronic vacuum polarization contribution to 
the effective charge $1 / \bar{\alpha}\left(m_{Z}^{2}\right)$ is shown for three cases, $\delta_{\alpha}=-0.1$ (a), 0 (b), +0.1 (c). The dotted lines are obtained by the approximate formula (6.6). The degree of freedom is 19 .

Fig.29 Total $\chi^{2}$ of the SM fit to all the electroweak data including the new left-right asymmetry data [31] as functions of $m_{H}$ for $m_{t}=100-200 \mathrm{GeV}$, for three selected $\alpha_{s}$ values (a) $\alpha_{s}\left(m_{Z}\right)=0.11$, (b) $\alpha_{s}\left(m_{Z}\right)=0.11$ and (c) $\alpha_{s}\left(m_{Z}\right)=0.11$, at $\delta_{\alpha}=0$. The dotted lines show our approximation (6.6) obtained by fitting the $\chi^{2}$ values in the region $63 \mathrm{GeV}<m_{H}<1000 \mathrm{GeV}$. The degree of freedom is 19 .

Fig.30 2-parameter fits to the $Z$ boson parameters, where in (a) $\bar{s}^{2}\left(m_{Z}^{2}\right)$ and $\bar{g}_{Z}^{2}\left(m_{Z}^{2}\right)$ are free parameters, and in (b) $S$ and $T$ are free parameters. In both cases the $Z b_{L} b_{L}$ vertex correction is assumed to be dominated by the SM contribution, and the $m_{t}$ value in the vertex correction is treated as external parameter in the fit. The 1$\sigma$ contours are shown for three representative $\alpha_{s}\left(m_{Z}\right)$ values, 0.11 (dashed lines), 0.12 (solid lines), 0.13 (dot-dashed lines). Also shown are the SM predictions in the range $100 \mathrm{GeV}<m_{t}<200 \mathrm{GeV}$ for $1 \mathrm{GeV}<m_{H}<1000 \mathrm{GeV}$ (a), and for $50 \mathrm{GeV}<m_{H}<1000 \mathrm{GeV}(\mathrm{b})$. The SM predictions in (a) and the $1-\sigma$ contours in (b) are obtained by assuming $\delta_{\alpha} \equiv 1 / \bar{\alpha}\left(m_{Z}^{2}\right)-128.72=0$.

Fig.31 Electroweak constraints on $\left(m_{t}, m_{H}\right)$ in the minimal SM, including the new left-right asymmetry data 31] and the constraint $m_{t}=174 \pm 16$ [106, for three selected $\alpha_{s}$ values at $\delta_{\alpha}=0$. Dashed lines show the best $m_{t}$ values for a given $m_{H}$, and the solid contours are for $\chi^{2}=\chi_{\min }^{2}+1$ and $\chi^{2}=\chi_{\min }^{2}+4.61$. The minimum point of $\chi^{2}$ is marked by " $\times$ ". The region $m_{H}<63 \mathrm{GeV}$ is excluded by LEP experiments [101].

Fig.32 Constraints on the Higgs mass in the SM from all the electroweak data including the new left-right asymmetry data 31]. Here the top mass $m_{t}$ is considered as external parameter with negligible uncertainty. Upper (solid lines) and lower (dashed lines) bound of the Higgs mass at 95\% CL are shown as functions of $m_{t}$ for $\alpha_{s}\left(m_{Z}\right)=0.11,0.12,0.13$. The hadronic vacuum polarization contribution to the effective charge $1 / \bar{\alpha}\left(m_{Z}^{2}\right)$ is set by $\delta_{\alpha} \equiv 1 / \bar{\alpha}\left(m_{Z}^{2}\right)-128.72=0$.

Fig.33 The contributions to $\chi^{2}$ from each sector of the analysis in the SM: (a) from the $Z$ parameters including the new left-right asymmetry data [31], (b) from the low energy neutral current experiments and (c) the $m_{W}$ measurements. They are calculated as functions of $m_{H}$ for $m_{t}=100-200 \mathrm{GeV}$, at $\alpha_{s}\left(m_{Z}\right)=0.12$ and $\delta_{\alpha}=0$. The degree of freedom is 9 for the $Z$ parameters (a), 9 for the low energy neutral current experiments (b), and 1 for $m_{W}(\mathrm{c})$. 